Why are children sedentary: an examination using the International Study of Childhood Obesity, Lifestyle and the Environment

by

Allana LeBlanc

\begin{abstract}
A thesis submitted to the
Faculty of Graduate and Postdoctoral Studies

in partial fulfillment of the requirements for

the PhD degree in Population Health
\end{abstract}

\author{
Population Health \\ University of Ottawa \\ Ottawa, Ontario, Canada
}

(September, 2015)

(C)Allana LeBlanc, Ottawa, Canada, 2015 


\begin{abstract}
Physical inactivity and sedentary behaviour have been independently associated with a wide range of negative health indicators including obesity, poor cardio-metabolic health, and poor psychosocial health. The overarching objective of this research was to gain a better understanding as to why children are sedentary, and where we need to focus public health messages and interventions to reduce sedentary behaviour. Specifically, I aimed to provide insight on current awareness of sedentary behaviour guidelines; determine important correlates of total sedentary time (SED), and screen time (ST) in Canadian children; and understand correlates of SED and ST in a global context. The primary dataset used for this project was the International Study of Childhood Obesity, Lifestyle and the Environment (ISCOLE). Background work was completed to review current literature on knowledge and awareness of Canadian physical activity and sedentary behaviour guidelines (in all age groups), and to understand the representativeness of the ISCOLE dataset. In addition to the two background papers, this dissertation includes three manuscripts, all prepared for submission in scientific, peer-reviewed journals:
\end{abstract}

1. Manuscript 1: Canadian physical activity and screen time guidelines: do children know?

2. Manuscript 2: Correlates of objectively measured sedentary time and selfreported screen time in Canadian children 
3. Manuscript 3: Correlates of total sedentary time and screen time in 9-11 year-old children around the world: The International Study of Childhood Obesity, Lifestyle and the Environment

Overall, this work showed the majority of children around the world are accumulating large amounts of sedentary time, and exceeded current screen time guidelines. We found that the large majority of Canadian children are not aware of screen time guidelines; however, a greater proportion of children could identify physical activity guidelines. We also identified a number of correlates of SED, and ST in Canadian children, and in children around the world. The most common correlates included weight status, and access to electronics in the house. Taken together, this work suggests that public health messaging should focus on increasing awareness of screen time guidelines. While increasing awareness of the guidelines, messaging can be tailored to promoting healthy weight status, and reducing (or removing) children's access to electronic devices in hopes of reducing overall time spent sedentary. 


\section{Co-Authorship}

This work could not have been possible without many invaluable collaborations. Below is a list of author contributions for the manuscripts included in this dissertation.

Background Manuscript 1: MST conceived of the manuscript. AGL, MST and MD designed the review. AGL, TB, SD, MD, GF, ALC, NO, RER, JCS, and MST aided in data collection, analysis, and critically reviewed the paper. All authors approved of the final version of the manuscript.

Background Manuscript 2: AGL, MST, and PTK conceived of the manuscript and designed the review. All authors aided in data collection and analysis. All authors approved of the final manuscript.

Manuscript 1: AGL, PEL, and MST conceived of the manuscript. $A G L, C B, M M B$, and $G L$ completed data collection. AGL, JPC, PEL, and MST completed data analysis. All authors critically reviewed the paper and approved of the final manuscript.

Manuscript 2: AGL, STB, JPC, and MST conceived of the manuscript. AGL, CB, GL, and MMB completed data collection with the help of the ISCOLE Research Group. AGL, STB, JPC, PTK and MST completed data analysis. All authors critically reviewed the manuscript and approved of its final form. 
Manuscript 3: AGL, JPC, PTK, and MST conceived of the manuscript. Data collection was completed by the ISCOLE Research Group. AGL, STB, JPC, PTK, and MST completed data analysis. All authors critically reviewed the paper and approved of its final form. 


\section{Acknowledgements}

First and foremost, I would like to thank Dr. Mark Tremblay for his mentorship over the past five years. Mark you took a chance and asked me to come work with you at HALO, more than five years ago, and helped shaped my career along the way. From the first time I met you in the entrance of CHEO, you've continued to provide guidance across all areas of my life, and inspired me to do meaningful work. You've shown me such patience and have been supportive of (almost) all of my crazy ideas; including doing my PhD.

To all the other HALOites out there, you're my second family. I am grateful to have such an amazing group of people to work with, and to be lucky enough to call many of you close friends. The bond created within HALO is one that can only be achieved when you hit each other with hockey sticks, and I have no doubt these bonds will last a lifetime. \#TeamLoft

Both before, and during this journey, I have also been fortunate to work with some of the best pediatric researchers and public relations people in the business. You encouraged me to start on this journey and have been there to support me all the way through. This is especially true for those at CSEP and ParticipACTION who encouraged me to pursue my PhD from the start. Also a huge thank you to everyone from ISCOLE and Pennington Biomedical Research Centre, without whose mentorship I'd still be scratching my head over SAS errors. 
Finally l'd like to thank my friends and family. My family, especially Mom and Dad, have supported me through every crazy decision I've made so far in life. From living in three provinces on two coasts, to going back to school "one more time", their support and encouragement has never faltered, even as I lay claim to becoming Governor General. 


\section{Table of Contents}

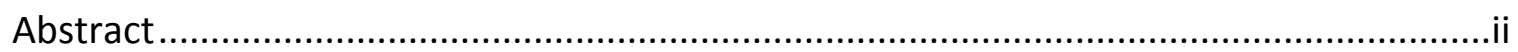

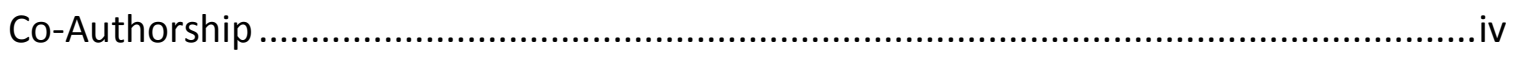

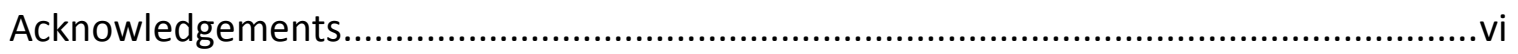

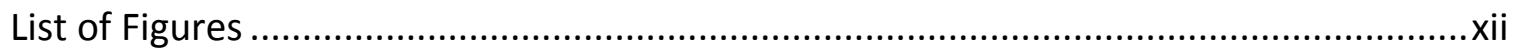

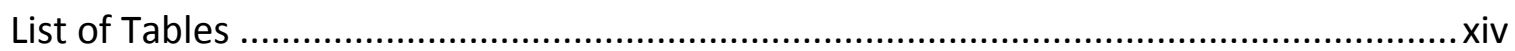

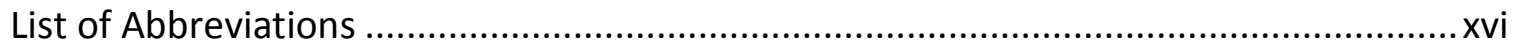

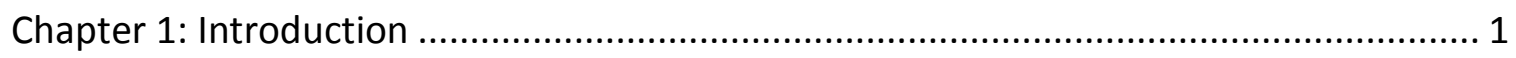

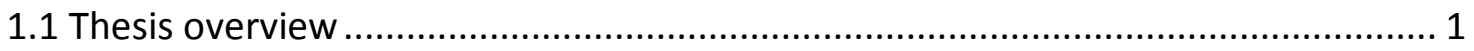

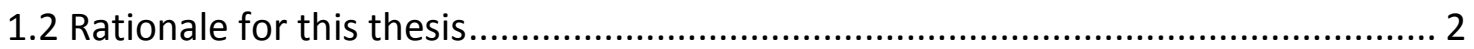

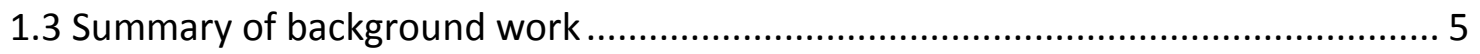

1.3.1 Background manuscript 1: Knowledge and awareness of Canadian Physical Activity and Sedentary Behaviour Guidelines: a synthesis of existing evidence ........ 5

1.3.2 Background manuscript 2: Are participant characteristics from ISCOLE study sites comparable to the rest of their country? ................................................... 6

1.3.3 Manuscript 1: Canadian physical activity and screen time guidelines: do

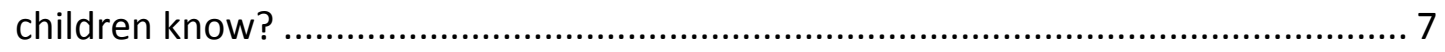

1.3.4 Manuscript 2: Correlates of objectively measured sedentary time and selfreported screen time in Canadian children ....................................................... 7

1.3.5 Manuscript 3:Correlates of total sedentary time and screen time in 9-11 yearold children around the world: The International Study of Childhood Obesity, Lifestyle and the Environment ...................................................................... 8 
1.4 Contributions of this work

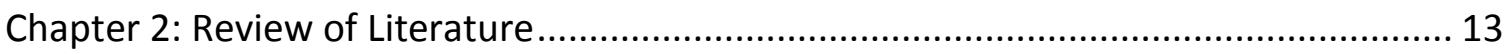

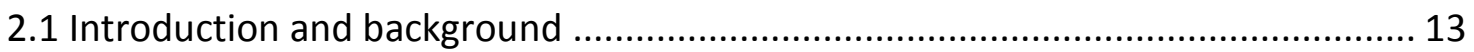

2.2 Physical activity and sedentary behaviour in population health .......................... 13

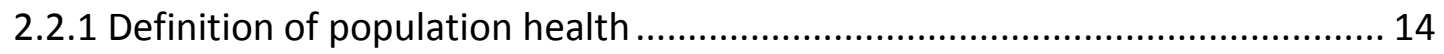

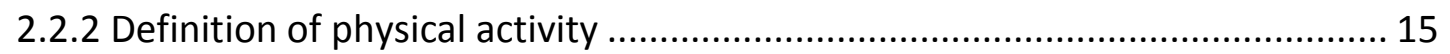

2.2.3 Definition of sedentary behaviour .......................................................... 15

2.3 Physical activity and sedentary behaviour in children and youth ....................... 16

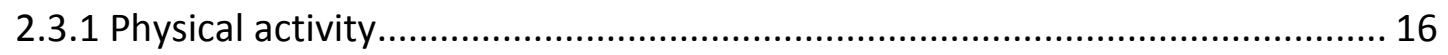

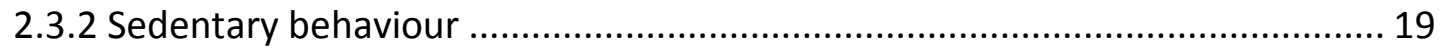

2.4 Canadian physical activity and sedentary behaviour guidelines ........................ 21

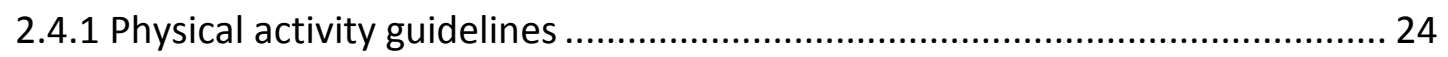

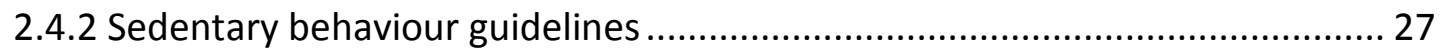

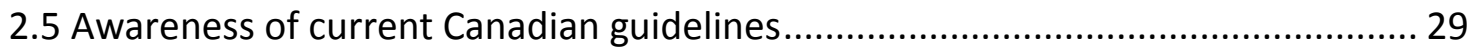

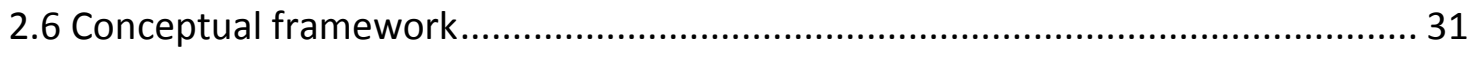

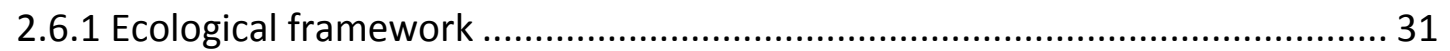

2.6.2 Determinants of active living and sedentary behaviour .............................. 33

2.7 Importance of studying sedentary behaviour in a population health context....... 38

2.7.1 Correlates of sedentary behaviour in a population health context ................ 40

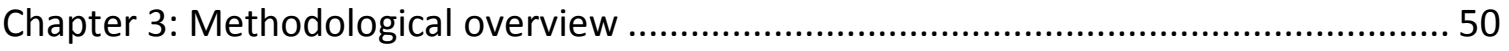

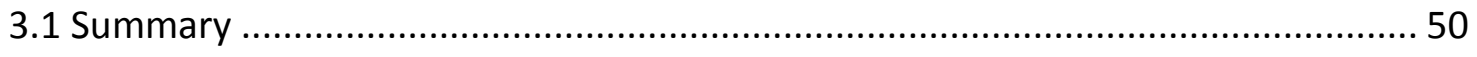

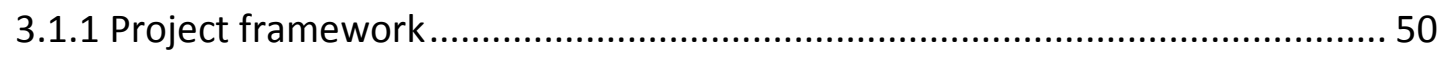

ix 
3.2 Summary of research methods and datasets

3.2.1 International Study of Childhood Obesity, Lifestyle and the Environment ..... 51

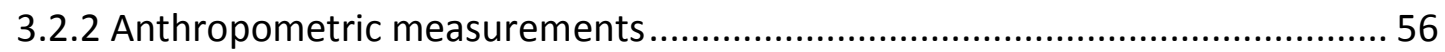

3.2.3 Directly measured physical activity and sedentary behaviour ...................... 58

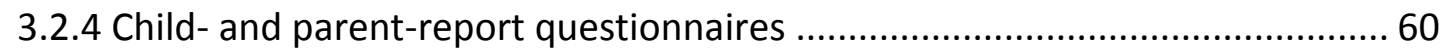

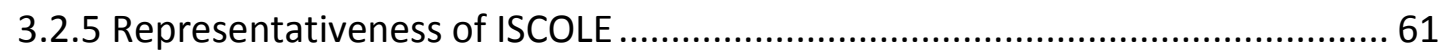

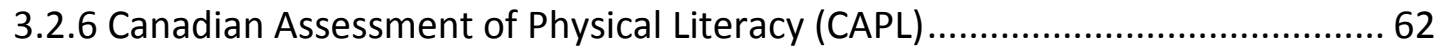

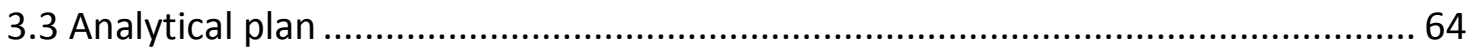

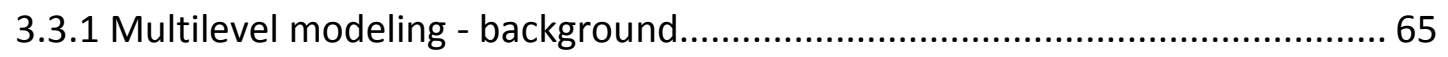

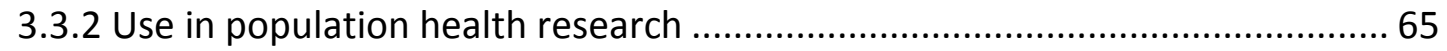

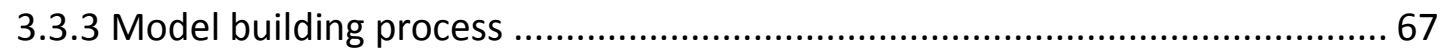

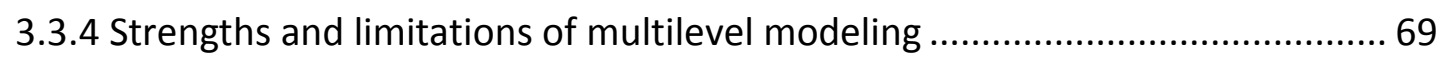

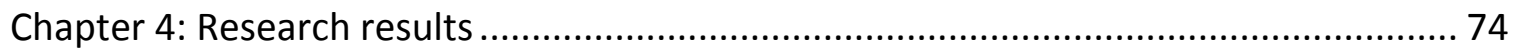

4.1 Canadian physical activity and screen time guidelines: are children aware?........ 74

4.2 Correlates of objectively measured sedentary time and self-reported screen time

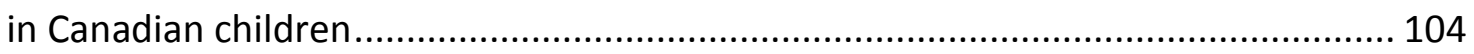

4.3 Correlates of total sedentary time and screen time in 9-11 year-old children around the world: The International Study of Childhood Obesity, Lifestyle and the

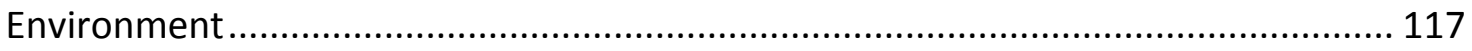

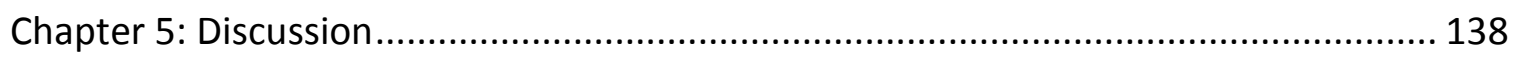

5.1 Summary of research findings................................................................. 138

5.2 Interpretations within a population health context ..................................... 140 
5.2.1 Continue to monitor and evaluate indicators of healthy active living

5.2.2 Harmonize data collection strategies around the world.

5.2.3 Ensure timely updates to guidelines and policies

5.2.4 Strive for continual improvement of physical activity and sedentary behaviour guidelines and messaging.

5.2.5 Refine methods for measuring sedentary behaviour in children and youth 149

5.3 Summary of strengths and limitations of this work

5.3.1 Strengths.

5.3.2 Limitations 153

5.4 Knowledge translation strategy 155

5.5 Conclusions 156

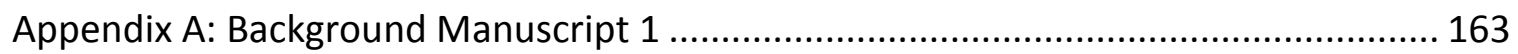

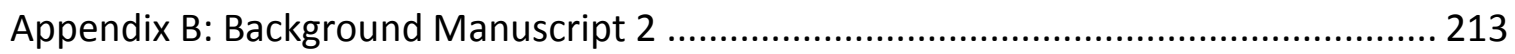

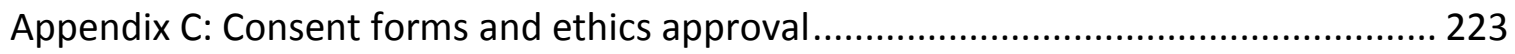

Appendix D: ISCOLE Diet and Lifestyle Questionnaire .......................................... 244

Appendix E: ISCOLE Demographic and Family Health Questionnaire .......................... 254

Appendix F: ISCOLE Neighbourhood \& Home Environment Questionnaire ................. 260

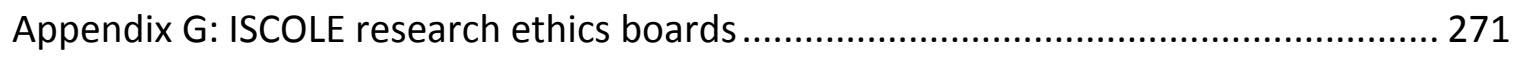

Appendix H: CAPL physical activity knowledge questionnaire ................................... 272

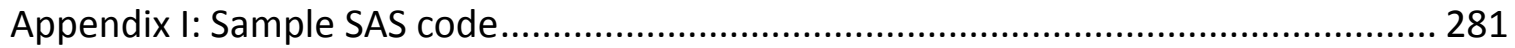




\section{List of Figures}

\section{Chapter 2}

Figure 2.1. Components of Population Health Research

Figure 2.2. Daily physical activity (steps per day) from ISCOLE study sites and their representative countries

Figure 2.3. Proportion of children exceeding screen time guidelines ( $>2$ hours per day) from ISCOLE sites and their representative countries

Figure 2.4. Canadian Physical Activity Guidelines for Children (aged 5-11 years)

Figure 2.5. Canadian Sedentary Behaviour Guidelines for Children (aged 5-11 years)

Figure 2.6. Ecological model of active living

Figure 2.7. Ecological model of sedentary behavior

Figure 2.8. Description of mediators, confounders, and moderators

\section{Chapter 3}

Figure 3.1. ISCOLE data collection sites around the world

\section{Chapter 4}

\section{Manuscript 1}

Figure 1. Average moderate- to vigorous-intensity physical activity (MVPA), total sedentary time, and knowledge of physical activity $(A)$ and screen time guidelines (B) 
Manuscript 3

Figure 1. Participants with missing data

Figure 2. Mean total sedentary time (A) and screen time score (B) 


\section{List of Tables}

\section{Chapter 2}

Table 2.1. Canadian physical activity and sedentary behaviour guidelines

\section{Chapter 3}

Table 3.1. ISCOLE country and participant characteristics

Table 3.2. Sampling methods employed by International Study of Childhood Obesity, Lifestyle and the Environment (ISCOLE) sites.

Table 3.3. ISCOLE anthropometric measurement table

Table 3.3. SAS output to calculate interclass correlation coefficients

Table 3.5. Model building process

\section{Chapter 4}

\section{Manuscript 1}

Table 1.Descriptive participant characteristics

Table 2.Responses to the Knowledge and Understanding of physical activity questionnaire and between-sex differences (total sample $n=1,010$ )

Table 3.Associations between habitual activity and perceptions of physical activity

\section{Manuscript 2}

Table 1. Potential correlates of objectively measured sedentary time and selfreported sedentary behavior

Table 2. Participant characteristics 
Table 3. Univariate correlates of total sedentary time

Table 4. Univariate correlates of self-reported screen time

Table 5. Socio-ecological domains and final models for accelerometer measures sedentary time and self-reported screen time

\section{Manuscript 3}

Table 1. Potential correlates of objectively measured sedentary time and selfreported screen time

Table 2. Descriptive characteristics of all participants

Table 3. Univariate correlates of total sedentary time

Table 4. Univariate correlates of total screen time

Table 5. Final model for correlates of accelerometer measured total sedentary time and self-reported screen time

Table 6. Significant correlates by ISCOLE country site for total sedentary time and screen time 


\section{List of Abbreviations}

\begin{tabular}{|c|c|}
\hline BMI & Body Mass Index \\
\hline CAPL & Canadian Assessment of Physical Literacy \\
\hline CFLRI & Canadian Fitness and Lifestyle Research Institute \\
\hline CSEP & Canadian Society for Exercise Physiology \\
\hline HALO & Healthy Active Living and Obesity Research Group \\
\hline \multirow[t]{2}{*}{ ISCOLE } & International Study of Childhood Obesity, Lifestyle and the \\
\hline & Environment \\
\hline MVPA & Moderate- to vigorous-intensity physical activity \\
\hline PA & Physical activity \\
\hline PHAC & Public Health Agency of Canada \\
\hline SB & Sedentary behavior \\
\hline SED & Total sedentary time \\
\hline ST & Screen time \\
\hline TV & Television \\
\hline U.K. & United Kingdom \\
\hline U.S. & United States \\
\hline WHO & World Health Organization \\
\hline
\end{tabular}




\section{Chapter 1}

\section{Introduction}

\subsection{Thesis overview}

This thesis aimed to contribute to our understanding of why children are sedentary. Data for this research came primarily from the International Study of Childhood Obesity, Lifestyle and the Environment (ISCOLE). Results of this work are presented within a Canadian context and are prepared for use by researchers, health care providers, and those working in governmental, non-governmental and not-forprofit organizations. It is hoped that this work can be used beyond those in academia to help inform updates to current Canadian physical activity and sedentary behaviour guidelines, to advise population health interventions, and to provide suggestions for future research.

Throughout this work, the following definitions were used: physical activity refers to any bodily movement produced by skeletal muscles that requires energy expenditure; ${ }^{1}$ physical inactivity refers to the absence of recommended levels of physical activity or exercise, usually measured by not meeting prescribed physical activity guidelines. Sedentary behaviour is defined as any waking behaviour that requires little to no energy expenditure (i.e. $\leq 1.5 \mathrm{MET}$ ) and occurs in a sitting or reclined position. ${ }^{2}$ Additional details on these terms can be found in the review of literature presented in Chapter 2. 
Chapter 1 presents a broad overview of the work that has been included as part of this thesis, and provides context and guidance for the reader. Chapter 2 provides a review of literature to help inform the reader, and to provide justification for why this work was needed. Chapter 3 provides methodological details, and gives an overview and rationale of statistical techniques used throughout this work. Chapter 4 presents the results of the three research articles that were completed as part of this dissertation. Chapter 5 summarizes the results and offers suggestions for future work. The appendices provide details on data collection (e.g., child- and parent-report questionnaires), data analysis (sample SAS code), research ethics approvals, and two background manuscripts that were used to help inform this work.

\subsection{Rationale for this thesis}

This work was inspired by the fact that low levels of physical activity (PA) and high levels of sedentary behaviour (SB) have become "normal" in our society. Both low PA and high SB levels are independently associated with a wide range of negative health indicators including overweight/obesity, poor cardio-metabolic health, poor psychosocial health, and increased risk for many diseases in children ${ }^{3-6}$ and adults. ${ }^{7,8}$ Further, when poor lifestyle habits are established at a young age, they are more likely to follow an individual through adolescence and into adulthood, putting them at increased risk for adverse health. ${ }^{9,10}$ Therefore, monitoring habitual PA and SB among children, as well as identifying their demographic, socioeconomic, and environmental determinants, represent a particularly important area of research. Previous work has focused largely on understanding different types and correlates of PA, and only recently 
have researchers turned their attention to the complex nature of SB. Therefore, it was

SB, and specifically screen time (e.g., watching television (TV), playing on the computer), that was of most interest to me.

Guided in part by the socio-ecological model, the overarching objective of this dissertation project was to gain a better understanding of correlates of SBs in children and youth. ${ }^{11}$ The conceptual frameworks outlined by Owen et al., ${ }^{11}$ on determinants of SB, and by Sallis et al., ${ }^{12}$ on determinants of active living, were used to understand the interactions between lifestyle, neighbourhood, and environmental factors contributing to high levels of sedentarism in children. The socio-ecological model was chosen to help guide this work because it takes into consideration the relationships between different environmental interactions and influences, and individual behaviours.

\section{Defining this work}

This dissertation includes a total of five manuscripts, all submitted for publication in scientific, peer-reviewed journals. Background work was completed to review current literature on knowledge and awareness of Canadian PA and SB guidelines, and to understand the representativeness of the ISCOLE dataset across each ISCOLE country. Below is an abbreviated abstract of each manuscript. Where applicable, the target journals have been included. The rationale for each manuscript was as follows:

Background manuscripts: The first background manuscript was completed to synthesize current knowledge and awareness of Canadian PA and SB guidelines. To date, little work has been done to assess guideline knowledge, and not a single study 
examined children's knowledge of the guidelines. This background manuscript supported the need to complete Manuscript 1. The second background manuscript was designed to understand if data collected from ISCOLE study sites were representative of the whole country. ISCOLE was not designed to be nationally representative; however, understanding how the ISCOLE study population differed from a representative sample from each country was very helpful when interpreting findings and developing public health recommendations. This manuscript was also designed to aid in increasing and updating my knowledge of similar studies around the world.

Manuscript 1: This manuscript was developed based on the assumption that public health messages to improve habitual behaviours are only beneficial if they are known to the general public; therefore, children who were aware of current PA guidelines and/or screen time (ST) guidelines would demonstrate more beneficial lifestyle habits (i.e., increased PA, decreased ST) than children who were unaware of the guidelines. If our assumptions were true, this would support a population health intervention aimed only at those who were unaware of current guidelines. If our assumptions were incorrect, and no differences in habitual activity existed, then population health interventions should be more widespread. This information could also be used when developing and disseminating updated guidelines.

Manuscript 2: This manuscript was developed to better understand correlates of total sedentary time (SED) and ST in Canadian children. Few studies have examined correlates of SED and ST in the same population, and no studies have examined these in 
a Canadian context. It was hoped that results from this study could be used to help inform updates to Canadian SB guidelines.

Manuscript 3: This manuscript was developed to expand on the results obtained in Manuscript 2, and to understand if correlates of SED and/or ST were similar across all 12 ISCOLE countries. It was assumed that correlates across all 12 ISCOLE countries would be similar to those identified in the Canadian sample. This work was unique in that no study has used such a large, geographically, and socio-culturally diverse sample to understand correlates of children's SB. This work can help international organizations develop public health messages and coordinate efforts to reduce SED and ST around the world.

\subsection{Summary of background work}

Below is a brief summary of each manuscript included in this dissertation. Full articles can be found in Chapter 4, with the exception of the background manuscripts, which can be found in the appendices.

\subsubsection{Background manuscript 1: Knowledge and awareness of Canadian Physical Activity and Sedentary Behaviour Guidelines: a synthesis of existing evidence} This manuscript has been published in the journal of Applied Physiology, Nutrition, and Metabolism. ${ }^{13}$ The aim of this review was to consolidate and synthesize existing evidence regarding current knowledge and awareness of Canadian PA and SB guidelines. Scientific databases, journal websites, content experts, organizations devoted to healthy active living, and government websites were searched for information pertaining to the guidelines. Overall, we found that awareness of the guidelines was low, especially with 
respect to the SB guidelines. Less than $10 \%$ of survey respondents from the Canadian population were aware of PA guidelines, and less than $5 \%$ were aware of SB guidelines. Online metrics showed that online accession of the guidelines was high (e.g., all "highly accessed" on journal websites). This review showed that awareness of Canadian PA and SB guidelines is low amongst the general population, but higher amongst the scientific and stakeholder community. Governmental, non-governmental, and stakeholder organizations should collaborate in creating sustained, long-term and well-resourced communication plans to reach the Canadian population to raise awareness of PA and SB guidelines and implement programs to facilitate their uptake.

\subsubsection{Background manuscript 2: Are participant characteristics from ISCOLE study sites} comparable to the rest of their country?

This manuscript has been submitted for publication in a special issue of the International Journal of Obesity. The purpose of this analysis was to examine the similarities and differences between participant characteristics from ISCOLE sites and data from nationally representative surveys from ISCOLE countries (Australia, Brazil, Canada, China, Colombia, Finland, Kenya, India, Portugal, South Africa, the United Kingdom, and the United States). Distributions of characteristics were assessed within each ISCOLE country-level database, and compared to published data from national or regional surveys, where available. Of 12 countries, data on weight status (BMI) were available in eight countries, data on measured PA (steps per day) were available in five countries, and data on self-reported ST were available in nine countries. Available country-specific data often used different measurement tools or cut-points, making 
direct comparisons difficult. From the analyses performed, the ISCOLE data do not seem to be systematically biased; however, due to limitations in data availability, data from ISCOLE should be used with appropriate caution when planning country-level population health interventions. This work highlights the need for harmonized measurement tools around the world while accounting for culturally specific characteristics, and the need for collaboration across study centres and research groups.

\subsubsection{Manuscript 1: Canadian physical activity and screen time guidelines: do children}

\section{know?}

This manuscript is under review at the Journal of School Health. This study aimed to determine the proportion of Canadian children who could correctly identify Canadian PA and ST guidelines. Data were obtained through the Canadian Assessment of Physical Literacy (CAPL) ( $n=787,46.6 \%$ boys) and a CAPL sub-sample of the International Study of Childhood Obesity, Lifestyle and the Environment (ISCOLE) ( $n=223,38.1 \%$ boys). Most children (71.6\%) correctly identified PA guidelines; $20.0 \%$ correctly identified ST guidelines. In adjusted models, children's ability to correctly identify guidelines did not differ by habitual activity. This study showed that regardless of habitual activity level, the majority of children correctly identified current PA guidelines; fewer children could identify ST guidelines.

\subsubsection{Manuscript 2: Correlates of objectively measured sedentary time and self- reported screen time in Canadian children}

This manuscript has been published, and is "highly accessed" in the International Journal of Behavioral Nutrition and Physical Activity, 2015; $12: 38 .{ }^{14}$ The objective of this paper 
was to identify correlates of SED and correlates of self-reported ST in Canadian children. Participants with complete demographic, anthropometric, and either SED ( $n=524,41 \%$ boys) or ST ( $n=567,42 \%$ boys) data from the Canadian ISCOLE site were included in analysis. Potential correlates of SED and ST were examined using multilevel general linear models, adjusting for sex, ethnicity, number of siblings, and socio-economic status with school as a random effect. Children averaged 8.5 hours of daily SED; no differences in total SED, or total ST were seen between girls and boys, but boys reported significantly more video game/computer usage than girls. In the final models, waist circumference and number of TVs in the home were the only common correlates of both SED and ST. Several factors were identified as correlates of SED and/or of ST in Canadian children; however, few correlates were common for both SED and ST, and for both boys and girls. This suggests that a single strategy to reduce SED and ST is unlikely to be effective. Future work should examine a variety of other non-screen based sedentary behaviours and their potential correlates in the hopes of creating tailored public health messages to reduce SED and ST in both boys, and girls.

\subsubsection{Manuscript 3: Correlates of total sedentary time and screen time in 9-11 year-old} children around the world: The International Study of Childhood Obesity, Lifestyle and the Environment

This manuscript has been published in PLOS ONE. The aim of this study was to investigate correlates of SED and ST in children from 12 countries around the world. The sample included 9-11 year-old children from ISCOLE study sites in Australia, Brazil, Canada, China, Colombia, Finland, India, Kenya, Portugal, South Africa, the United 
Kingdom, and the United States. Children with complete demographic, anthropometric, accelerometer determined SED, and self-reported ST data were included in this analysis ( $n=5,844,45.6 \%$ boys). Potential correlates of SED and ST were examined using multilevel general linear models, adjusting for sex, age, and highest parental education, with school and study site as random effects. Children averaged 8.6 hours of daily SED, and $54.2 \%$ of children exceeded current ST guidelines of $\leq 2$ hours/day. In all study sites, boys reported higher ST, were less likely to meet ST guidelines, and tended to have higher BMI z-scores than girls. In several study sites (9/12), girls engaged in significantly more SED than boys. Common correlates of higher SED and ST across the total sample included weight status, having a TV or a computer in the bedroom, and not meeting PA guidelines. Many common correlates of SED and ST were identified, some of which are easily modifiable (e.g., removing TV or computer from the bedroom), and others that may require more intense behavioral interventions (e.g., meeting physical activity guidelines).

\subsection{Contributions of this work}

This dissertation provides a comprehensive look at awareness of public health guidelines, and SB in children. We showed that among children, awareness of PA guidelines is high, but ST guidelines is low, and that their awareness was not associated with accelerometer measured physical activity or child-reported screen time. We also identified correlates of increased SED and ST in Canada, and around the world. We were able to identify some common correlates of SED and ST. Some of which may be easily modified through public health messaging, and others that may require more in depth 
behavioral interventions. Overall, several recommendations were made for future work including to continue to monitor and evaluate indicators of healthy active living; to harmonize data collection strategies; to ensure timely updates to guideline and policies; to strive for continual improvement of physical activity and sedentary behavior guidelines; and to refine measurements of SB. Results of this doctoral dissertation have been, and will be disseminated through peer reviewed publications, and conference presentations with additional outreach through various social media outlets. It is also hoped that the work presented herein may inform future research. 


\section{References}

1. Pate, R. \& Pratt, M. Physical activity and public health: A recommendation from the Centers for Disease Control and Prevention and the American College of Sports Medicine. JAMA273, 402-407 (1995).

2. Sedentary Behaviour Research Network. Letter to the Editor: Standardized use of the terms 'sedentary' and 'sedentary behaviours'. Appl. Physiol. Nutr. Metab.37, 540542 (2012).

3. Janssen, I. \& LeBlanc, A. G. Systematic review of the health benefits of physical activity and fitness in school-aged children and youth. Int. J. Behav. Nutr. Phys. Act.7, 40 (2010).

4. LeBlanc, A. G. et al. Systematic review of sedentary behaviour and health indicators in the early years (aged 0-4 years). Appl. Physiol. Nutr. Metab.37, 753-772 (2012).

5. Timmons, B. W. et al. Systematic review of physical activity and health in the early years (aged 0-4 years). Appl. Physiol. Nutr. Metab.37, 773-792 (2012).

6. Tremblay, M. S. et al. Systematic review of sedentary behaviour and health indicators in school-aged children and youth. Int. J. Behav. Nutr. Phys. Act.8, 98 (2011).

7. Paterson, D. \& Warburton, D. Physical Activity and Functional Limitations in Older Adults: A Systematic Review Related to Canada's Physical Activity Guidelines. Int. J. Behav. Nutr. Phys. Act.7, (2010). 
8. Warburton, D. E., Charlesworth, S., Ivey, A., Nettlefold, L. \& Bredin, S. S. A systematic review of the evidence for Canada's Physical Activity Guidelines for Adults. Int. J. Behav. Nutr. Phys. Act.7, 39 (2010).

9. Janssen, I. et al. Utility of Childhood BMI in the Prediction of Adulthood Disease: Comparison of National and International References. Obes. Res.13, 1106-1115 (2005).

10. Ruiz, J. R. et al. Predictive validity of health-related fitness in youth: a systematic review. Br. J. Sports Med.43, 909-923 (2009).

11. Owen, N. et al. Adults' Sedentary Behavior: Determinants and Interventions. Am. J. Prev. Med.41, 189-196 (2011).

12. Sallis, J. F., Owen, N. \& Fisher, E. B. in Health behavior and health education: Theory, research, and practice (4th ed.) (eds. Glanz, K., Rimer, B. K. \& Viswanath, K.) 465-485 (Jossey-Bass, 2008).

13. LeBlanc, A. G. et al. Knowledge, awareness, and uptake of Canadian physical activity and sedentary behaviour guidelines. Appl. Physiol. Nutr. Metab.39, 27 (2014). 14. LeBlanc, A. G. et al. Correlates of objectively measured sedentary time and selfreported screen time in Canadian children. Int. J. Behav. Nutr. Phys. Act.12, 38 (2015). 


\section{Chapter 2}

\section{Review of literature}

\subsection{Introduction and background}

This Chapter aims to prepare the reader with adequate background information to interpret the importance of the manuscripts presented in this dissertation. Here I provide the comprehensive definitions of terms used throughout this work, current PA and SB guidelines, an overview of the health implications of a sedentary lifestyle, the conceptual framework that has informed the development of this work, and the importance of understanding correlates of SB to inform future interventions.

Although this work will focus primarily on SED and ST, it would be impossible to provide the reader with adequate background information without also acknowledging the importance of PA. Engaging in habitual PA is widely accepted as an effective preventative measure for a variety of health risk factors across all age, gender, ethnic, and socioeconomic subgroups. ${ }^{1-3}$ However, across all age groups, levels of PA remain low ${ }^{4,5}$ and levels of sedentarism continue to rise. ${ }^{4-6}$ Taken together, these lifestyle habits threaten the persistent increase in life expectancy enjoyed over the past century. $^{7}$

\subsection{Physical activity and sedentary behaviour in population health}

With the explosion of research in the field of SB over recent years, and with the wealth of information available on PA research, it is important to provide context as to how these terms have been used throughout this work. Chapter 1 provided a brief 
definition of PA and SB, but to help guide the reader, the following comprehensive definitions are provided for population health, PA, and SB.

\subsubsection{Definition of population health}

Population health is a relatively new term and can be described as an approach that aims to shift from a focus on health care for individuals who are ill, to improving the health of an entire population, by treating health as a state of complete physical, mental and social well-being, and by reducing health inequities among population groups. ${ }^{8-10}$ Kinding and Stoddart suggest a framework (Figure 2.1) to better understand the components of population health. ${ }^{9}$ This is included to show how policies or interventions that are suggested within this dissertation can have an effect on a broader population level.

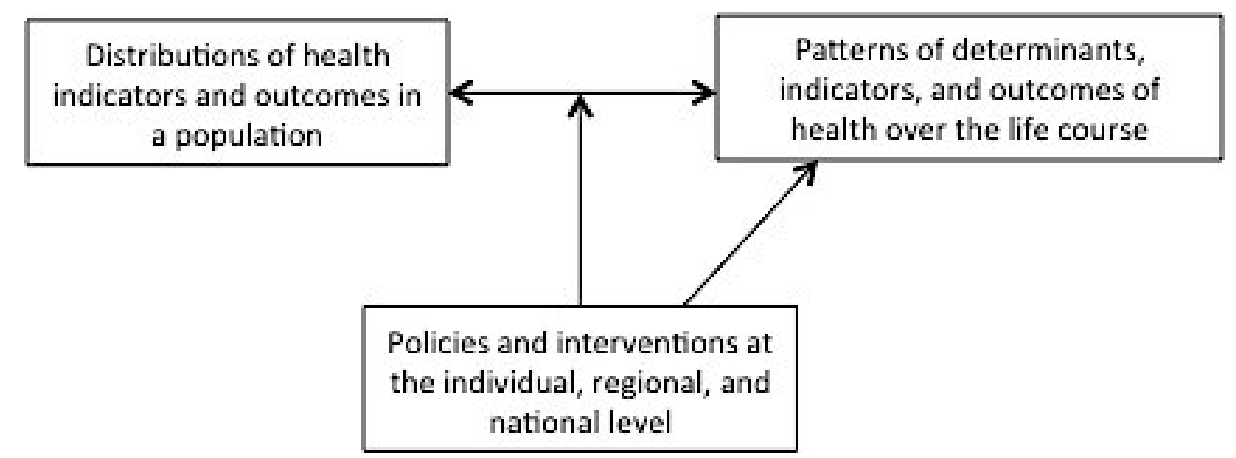

Figure 2.1. Components of Population Health Research adapted from Kinding and

\section{Stoddart ${ }^{9}$}

Within population health, there are clearly many different research foci. In 2012, The Lancet devoted a special issue to PA and public health research, bringing it into the spotlight. ${ }^{11} \mathrm{PA}$ and public health research require coordinated efforts by many different scientific disciplines including epidemiology, exercise (and now sedentary) physiology, 
environmental and health science, sociology, and urban planning. ${ }^{11}$ The framework proposed by Kinding and Stoddart helps to illustrate how an interdisciplinary approach may work to change policies that may ultimately improve health.

\subsubsection{Definition of physical activity}

PA refers to any bodily movement produced by skeletal muscles that requires energy expenditure; ${ }^{12}$ physical inactivity refers to the absence of recommended levels of PA or exercise, usually measured by not meeting prescribed PA guidelines described below and in Table 2.1 and included as Figure 2.4. PA is usually prescribed using the FITT principle (Frequency, Intensity, Time, and Type $)^{13}$ and in accordance with PA guidelines. ${ }^{14}$ The FITT principle and current Canadian PA guidelines are described below.

\subsubsection{Definition of sedentary behaviour}

In recent years, we have seen a proliferation of research related to the health impacts of SB, and sedentary physiology has become a distinct field of study. ${ }^{15}$ Recently, the SB Research Network proposed a standardized definition of the terms "sedentary" and "sedentary behaviour". ${ }^{16}$ These definitions have become common in the literature, and are what was used to inform this work. They defined SB as "any waking behaviour that requires little to no energy expenditure (i.e. $\leq 1.5 \mathrm{METs}$ ) and occurs in a sitting or reclined position".${ }^{16}$ Total sedentary time can be further classified by a variety of activities such as reading, watching television, or using the computer. Similarly to PA, SB is prescribed using the SITT principle (Sedentary behaviour, Interruptions, Time, and Type) ${ }^{15}$ and in accordance with SB guidelines (described below and in Table 2.1 and Figure 2.5). ${ }^{17}$ The SITT principle and current Canadian SB guidelines are described below. 
For this work, three terms will be used to describe sedentarism: SB, total sedentary time (SED), and screen time (ST).

- Sedentary behaviour (SB) as defined by the Sedentary Behaviour Research Network, includes all behaviours that require little-to-no energy expenditure (i.e., $\leq 1.5 \mathrm{METs}$ ) that occur in the sitting or reclined position. ${ }^{16} \mathrm{SB}$ can be directly measured (e.g., via accelerometers or inclinometers), self-reported, or a combination of many different measurement tools.

- Total sedentary time (SED) includes total daily sedentary time measured via accelerometry, as determined by widely used cut-points. ${ }^{18}$ SED does not distinguish between discretionary time and time at school (or work) and is associated with limitations of accelerometry (discussed later).

- Screen time (ST) includes any time children are engaging in sedentary, screen based activities, including watching TV, playing seated video games, and using the computer during their free time. For this dissertation, ST was measured via child-report questionnaire. ST does not include time children spend using screens at school, or for homework, or the use of active video games.

\subsection{Physical activity and sedentary behaviour in children and youth}

\subsubsection{Physical activity}

Current data suggest that a large majority of children around the world are not meeting current PA guidelines from Canada, ${ }^{14}$ the U.S., ${ }^{19}$ the U.K., ${ }^{20}$ Australia, ${ }^{21}$ and the World Health Organization. ${ }^{22}$ For example, self-report measures from the Global Schoolbased Student Health Survey and the Health Behaviour in School-aged Children Study 
conducted in 38 European countries, reported that only $20 \%$ of $13-15$ year olds participated in at least 60 minutes of moderate- to vigorous-intensity physical activity (MVPA) daily. ${ }^{23}$ Accelerometer data from the Canadian Health Measures Survey suggest that only $7 \%$ of children and youth aged $6-19$ years participate in at least 60 minutes of MVPA per day. ${ }^{4}$ This is consistent with Background Manuscript 2, which showed that across all 12 study sites from the International Study of Childhood Obesity, Lifestyle and the Environment (ISCOLE), few children met current guidelines of 12,000 steps per day (Figure 2.1, with additional details provided in Background Manuscript 2 in Appendix A). 


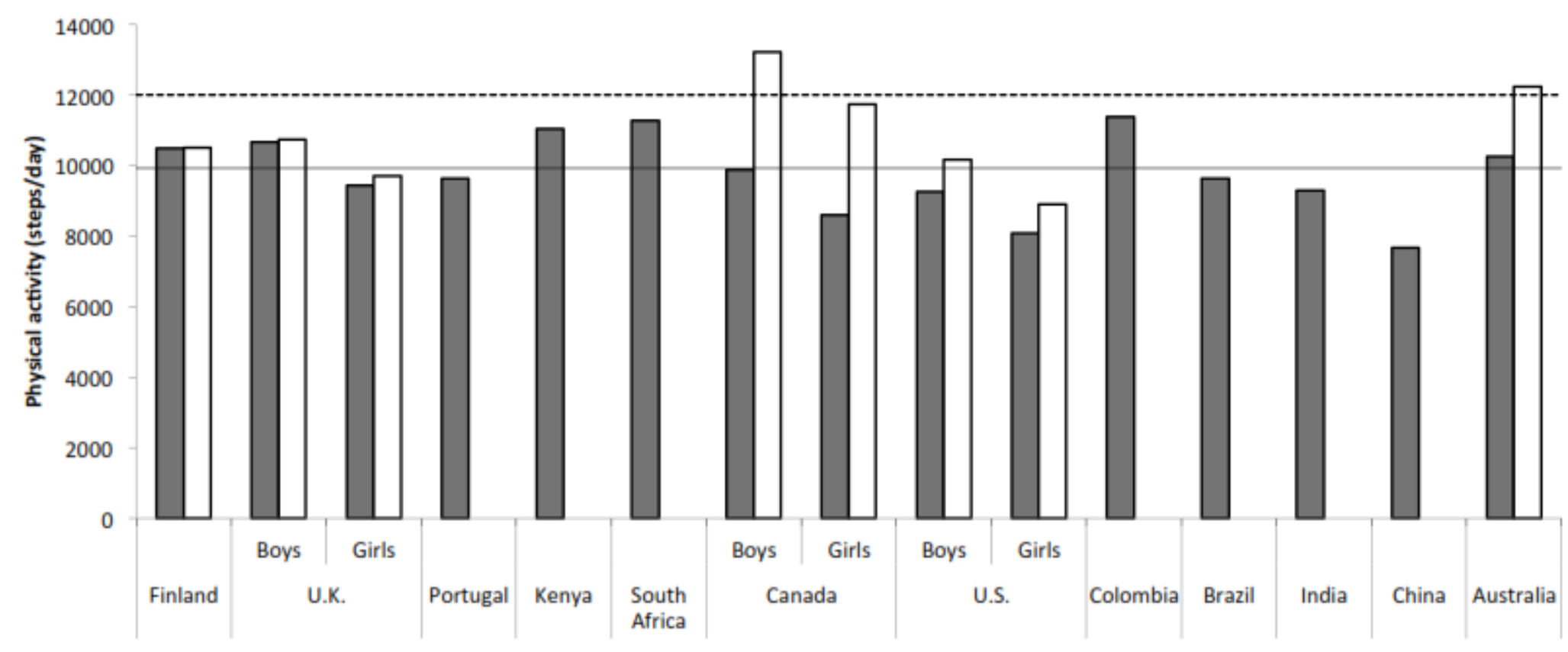

ISCOLE site country

Figure 2.2. Daily physical activity (steps per day) from ISCOLE study sites and their representative countries.

Legend: Dark grey bars indicate data from ISCOLE participants; white bars represent country-level data. If no white bar, then country level data were not available. Where available, data are presented for both boys and girls. Horizontal black line represents mean steps per day for all ISCOLE participants; horizontal dashed line represents target of 12,000 steps per day recommended to meet current physical activity guidelines. ${ }^{24}$ Data were included if it was collected via pedometer or accelerometer, and presented as sample mean. Country level datasets included: Finland: Physical Activity of School Aged Children; ${ }^{25}$ United Kingdom: Millennium Cohort Study; ${ }^{26}$ Canada: Canadian Health Measures Survey; ${ }^{4}$ U.S.: National Health and Nutrition Examination Survey; ${ }^{27}$ Australia: Australian National Children's Nutrition and Physical Activity Survey. ${ }^{28}$ The Millennium Cohort Study also provided data for England: 10,147 steps per day compared to 9,982 steps per day in ISCOLE. See Background Manuscript 2 for additional details. 


\subsubsection{Sedentary behaviour}

Accelerometer results from the Canadian Health Measures Survey report that Canadian boys, aged 6-19 years are spending an average of 8.5 hours being sedentary per day; girls aged 6-19 years are spending 8.7 hours being sedentary per day. ${ }^{4}$ These high levels of sedentarism are not unique to Canadian children. Objectively measured SED data from the National Health and Nutrition Examination Survey (NHANES) in the U.S. show that boys aged 6-19 years accumulate 6.0-7.9 hours of daily SED (with the oldest children being the most sedentary); girls were slightly more sedentary accumulating 6.1-8.1 hours daily for the same age groups (again with the oldest being the most sedentary). ${ }^{6}$ On average, across all 12 ISCOLE sites, $54.5 \%$ of children exceeded current screen time guidelines of no more than two hours of screen time per day (Figure 2.3). ${ }^{17}$ In many cases this was similar to what has been reported in other studies (see Background Manuscript 2 in Appendix A for additional details). 


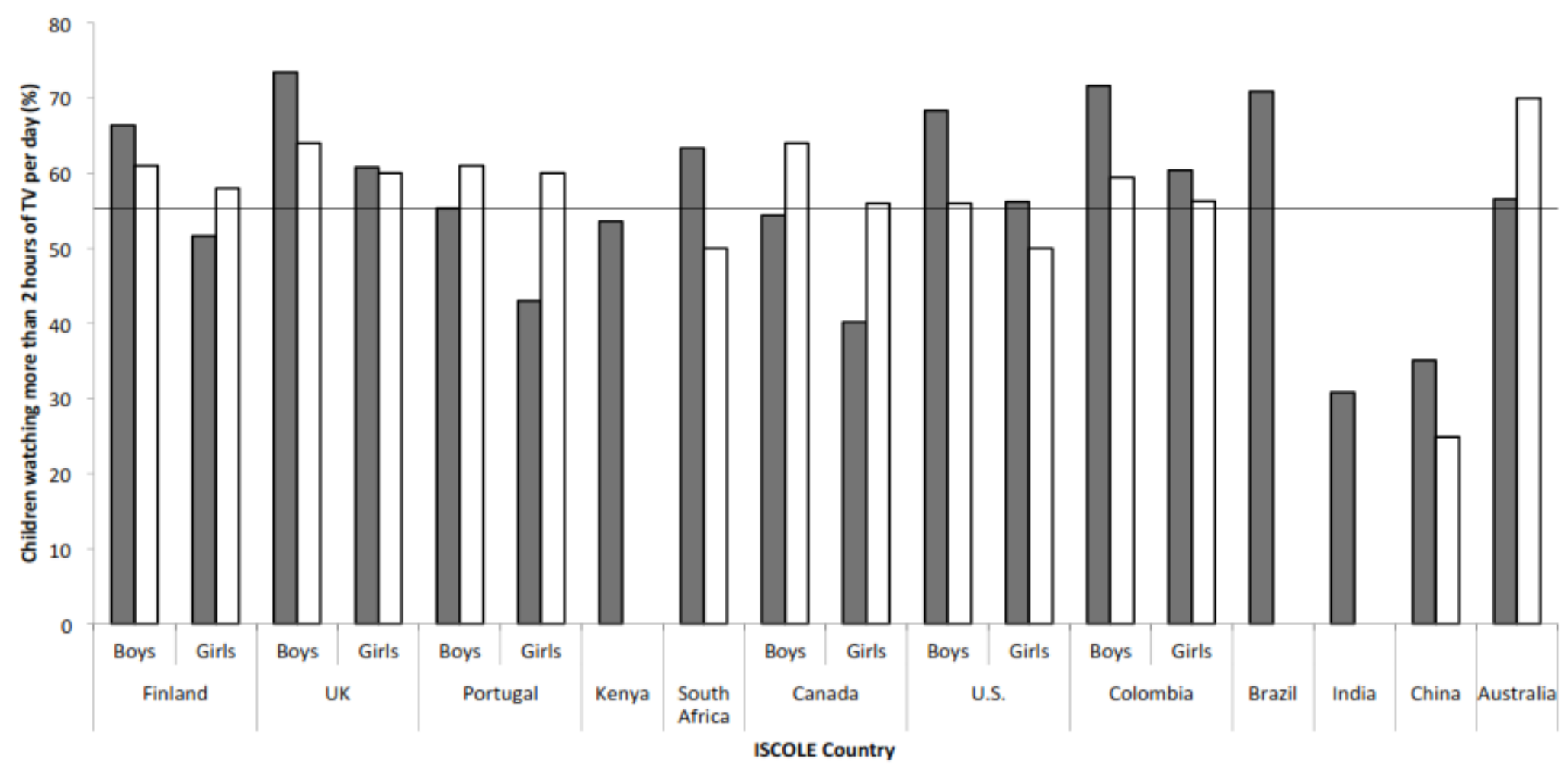

Figure 2.3. Proportion of children exceeding screen time guidelines ( $>2$ hours per day) from ISCOLE sites and their representative countries.

Legend: Dark grey bars indicate data from ISCOLE participants; white bars represent country-level data. If no white bar, then country level data were not available. Where available, data are presented for both boys and girls. Horizontal black line represents mean proportion of all ISCOLE participants who exceed screen time guidelines. Country level datasets included: Canada, Finland, the U.K., and the U.S.: Healthy Behaviors in School-aged Children; ${ }^{29}$ South Africa: Time of Use survey; ${ }^{30}$ Colombia: Instituto Colombiano de Bienestar Familiar (ICBF); China: China Health and Nutrition Survey; ${ }^{31}$ Australia: Australian Bureau of Statistics. ${ }^{32}$ See Background Manuscript 2 for additional details. 
With the widespread use of accelerometers, we are starting to understand the effects of long bouts on sedentarism on acute and chronic health conditions. In adults, long bouts of SED are associated with acute and chronic ill-health, regardless of the type of SB. ${ }^{33,34}$ In children, limiting SB is associated with better measures of body composition, fitness, self-esteem, self-worth, pro-social behaviour and academic achievement. ${ }^{35}$ However, previous reviews have been informed primarily by reports of screen-based behaviours. More recent studies using accelerometer data and markers of cardio-metabolic health have been less conclusive. Evidence from both clinical and population based studies have shown that in children, long bouts of SED are not associated with acute elevations in cardio-metabolic health risk, ${ }^{36} \mathrm{BMI}$, or waist circumference, ${ }^{37}$ thus raising the question that in children, if it is total SED or specific SBs that are associated with poor health. Currently, the relationship between SB and health seems to be at least partially mitigated by how SB is measured, defined, and categorized.

\subsection{Canadian physical activity and sedentary behaviour guidelines}

Current Canadian PA guidelines for children and youth were released in 2011 and have been fundamental in providing the public with evidence-informed targets to follow for health benefits, and have the potential to significantly contribute to improvements in overall health across Canada. ${ }^{14,38}$ All guidelines were informed by systematic reviews of the literature. The development of the guidelines has been recorded in detail and can be found on the Canadian Society for Exercise Physiology website (www.csep.ca/guidelines). Currently, SB guidelines exist only for the early years, 
children, and youth. No SB guidelines exist for adults or older adults in Canada, or elsewhere. Table 2.1 provides an overview of PA guidelines for all age groups and SB guidelines for the early years, children, and youth, and outlines the associated health benefits of meeting the guidelines for all age groups. All groups were included to help highlight current gaps, and show that regardless of age group, following the guidelines is associated with good health.

Public facing information sheets for PA guidelines for children (aged 5-11 years) are presented below in Figure 2.4; information sheets for SB for children (aged 5-11 years) are presented in Figure 2.5. Guidelines for youth (aged 12-17 years) are the same as the guidelines for children; however, the public facing messaging and information sheets are slightly different. For the purpose of this work, the guidelines and messaging for children are most relevant.

Table 2.1. Canadian physical activity and sedentary behaviour guidelines.

\begin{tabular}{|c|c|c|c|}
\hline Physical activity Guideline* & $\begin{array}{l}\text { Associated } \\
\text { health benefit }\end{array}$ & $\begin{array}{l}\text { Sedentary behaviour } \\
\text { guideline }\end{array}$ & $\begin{array}{l}\text { Associated health } \\
\text { benefit }\end{array}$ \\
\hline \multicolumn{4}{|l|}{ Early years (aged 0-4) } \\
\hline $\begin{array}{l}\text { Infants (aged less than } 1 \text { year) } \\
\text { should be physically active } \\
\text { several times daily - } \\
\text { particularly through } \\
\text { interactive floor-based play. } \\
\text { Toddlers (aged 1-2 years) and } \\
\text { preschoolers (aged 3-4 years) } \\
\text { should accumulate at least } \\
180 \text { minutes of physical } \\
\text { activity at any intensity spread } \\
\text { throughout the day, including: } \\
\text { - A variety of activities } \\
\text { in different } \\
\text { environments } \\
\text { - Activities that } \\
\text { develop movement } \\
\text { skills }\end{array}$ & $\begin{array}{l}\text { In this age } \\
\text { group, higher } \\
\text { levels of physical } \\
\text { activity are } \\
\text { associated with } \\
\text { improved } \\
\text { measures of } \\
\text { adiposity, motor } \\
\text { skill } \\
\text { development, } \\
\text { cognitive } \\
\text { development, } \\
\text { psychosocial } \\
\text { health, bone and } \\
\text { skeletal health, } \\
\text { and indicators of } \\
\text { cardio-metabolic } \\
\text { health. }{ }^{40}\end{array}$ & $\begin{array}{l}\text { For healthy growth and } \\
\text { development, caregivers } \\
\text { should minimize the time } \\
\text { infants (aged less than } 1 \\
\text { year), toddlers (aged 1-2 } \\
\text { years), and preschoolers } \\
\text { (aged 3-4 years) spend } \\
\text { being sedentary during } \\
\text { waking hours. This } \\
\text { includes prolonged } \\
\text { sitting or being } \\
\text { restrained (e.g., stroller, } \\
\text { high chair) for more than } \\
\text { one hour at a time. } \\
\text { For those under } 2 \text { years, } \\
\text { screen time (e.g., TV, } \\
\text { computer, electronic }\end{array}$ & $\begin{array}{l}\text { Limiting sedentary } \\
\text { behaviour (especially } \\
\text { screen time) in the } \\
\text { early years is } \\
\text { associated with } \\
\text { better measures of } \\
\text { adiposity, } \\
\text { psychosocial health, } \\
\text { and cognitive } \\
\text { development. }\end{array}$ \\
\hline
\end{tabular}




\begin{tabular}{|c|c|c|c|}
\hline $\begin{array}{l}\text { Progression toward } \\
\text { at least } 60 \text { minutes of } \\
\text { energetic play by } 5 \\
\text { years of age. } \\
\text { More daily physical activity } \\
\text { provides greater benefits. }^{39}\end{array}$ & & $\begin{array}{l}\text { games) is not } \\
\text { recommended. } \\
\text { For children } 2-4 \text { years, } \\
\text { screen time should be } \\
\text { limited to under one } \\
\text { hour per day; less is } \\
\text { better. }{ }^{41}\end{array}$ & \\
\hline \multicolumn{4}{|c|}{ Children (aged 5-11 years) and youth (aged 12-17 years) } \\
\hline $\begin{array}{l}\text { For health benefits, children } \\
\text { aged 5-11 years, and youth } \\
\text { aged } 12-17 \text { years should } \\
\text { accumulate at least } 60 \\
\text { minutes of moderate- to } \\
\text { vigorous-intensity physical } \\
\text { activity daily. This should } \\
\text { include: } \\
\text { - Vigorous-intensity } \\
\quad \text { activities at least } 3 \\
\text { days per week } \\
\text { - Activities that } \\
\text { strengthen muscle } \\
\text { and bone at least } 3 \\
\text { days per week } \\
\text { More daily physical activity } \\
\text { provides greater health } \\
\text { benefits. }{ }^{14}\end{array}$ & $\begin{array}{l}\text { For children and } \\
\text { youth, higher } \\
\text { levels of physical } \\
\text { activity are } \\
\text { associated with } \\
\text { better measures } \\
\text { of cardio- } \\
\text { metabolic } \\
\text { health, adiposity, } \\
\text { bone health, and } \\
\text { mental health. } \\
\text { Further, } \\
\text { associated } \\
\text { health benefits } \\
\text { increase with } \\
\text { increasing levels } \\
\text { of activity, } \\
\text { especially when } \\
\text { the activity is } \\
\text { primarily aerobic } \\
\text { in nature. }{ }^{1}\end{array}$ & $\begin{array}{l}\text { For health benefits, } \\
\text { children (aged } 5-11 \\
\text { years) and youth (aged } \\
\text { 12-17 years) should } \\
\text { minimize the time they } \\
\text { spend being sedentary } \\
\text { each day. This may be } \\
\text { achieved by } \\
\text { - } \quad \text { Limiting } \\
\text { recreational } \\
\text { screen time to } \\
\text { no more than } 2 \\
\text { hours per day; } \\
\text { lower levels are } \\
\text { associated with } \\
\text { additional } \\
\text { health benefits } \\
\text { Limit sedentary } \\
\text { (motorized) } \\
\text { transport, } \\
\text { extended sitting } \\
\text { and time spend } \\
\text { indoors } \\
\text { throughout the } \\
\text { day. }{ }^{17} \\
\end{array}$ & $\begin{array}{l}\text { Reducing sedentary } \\
\text { behaviour (especially } \\
\text { via reduced screen } \\
\text { time) is associated } \\
\text { with better measures } \\
\text { of body composition, } \\
\text { cardiorespiratory and } \\
\text { musculoskeletal } \\
\text { fitness, academic } \\
\text { achievement, self- } \\
\text { esteem, and social } \\
\text { behaviours. }\end{array}$ \\
\hline \multicolumn{4}{|l|}{ Adults (aged $18-64$ years) } \\
\hline $\begin{array}{l}\text { To achieve health benefits, } \\
\text { adults aged } 18-64 \text { years } \\
\text { should accumulate at least } \\
150 \text { minutes of moderate- to } \\
\text { vigorous-intensity aerobic } \\
\text { physical activity per week, in } \\
\text { bouts of } 10 \text { minutes or more. } \\
\text { It is also beneficial to add } \\
\text { muscle and bone } \\
\text { strengthening activities using } \\
\text { major muscle groups, at least } \\
2 \text { days per week. } \\
\text { More daily physical activity } \\
\text { provides greater health } \\
\text { benefits. }{ }^{14}\end{array}$ & $\begin{array}{l}\text { For adults aged } \\
18-64 \text { years, } \\
\text { following the } \\
\text { guidelines is } \\
\text { associated with } \\
\text { significant } \\
\text { reductions in the } \\
\text { risk of all-cause } \\
\text { mortality, } \\
\text { cardiovascular } \\
\text { disease, stroke, } \\
\text { hypertension, } \\
\text { colon and breast } \\
\text { cancer, and type } \\
2 \text { diabetes. }\end{array}$ & None available & Not applicable \\
\hline
\end{tabular}




\begin{tabular}{|c|c|c|c|}
\hline \multicolumn{4}{|l|}{ Older adults (65 years and older) } \\
\hline $\begin{array}{l}\text { To achieve health benefits } \\
\text { and improve functional } \\
\text { abilities, adults aged } 65 \text { years } \\
\text { and older should accumulate } \\
\text { at least } 150 \text { minutes of } \\
\text { moderate- to vigorous- } \\
\text { intensity aerobic physical } \\
\text { activity per week, in bouts of } \\
10 \text { minutes or more. It is also } \\
\text { beneficial to add muscle and } \\
\text { bone strengthening activities } \\
\text { using major muscle groups, at } \\
\text { least } 2 \text { days per week. } \\
\text { Those with poor mobility } \\
\text { should perform physical } \\
\text { activities to enhance balance } \\
\text { and prevent falls. } \\
\text { More daily physical activity } \\
\text { provides greater health } \\
\text { benefits. }{ }^{14}\end{array}$ & $\begin{array}{l}\text { In older adults, } \\
\text { following the } \\
\text { guidelines is } \\
\text { inversely } \\
\text { associated with } \\
\text { major chronic } \\
\text { disease such as } \\
\text { coronary heart } \\
\text { disease, type } 2 \\
\text { diabetes, } \\
\text { depression, } \\
\text { certain cancers, } \\
\text { dementia, } \\
\text { disability, and } \\
\text { loss of function. }{ }^{3}\end{array}$ & None available & Not applicable \\
\hline
\end{tabular}

*Guidelines are presented verbatim with permission from CSEP from www.csep.ca/guidelines

\subsubsection{Physical activity guidelines}

Current Canadian PA Guidelines for Children and Youth recommend that children (aged 5-11 years) and youth (aged 12-17 years) accumulate 60 minutes of MVPA daily (Figure 2.3). The guidelines also suggest that children and youth incorporate vigorousintensity activities, and activities that strengthen muscle and bone, at least three days per week. ${ }^{14}$ Meeting the guidelines is associated with better measures of cardiometabolic health (i.e. blood pressure, cholesterol, and metabolic syndrome), overweight/obesity, bone health, and mental health. Associated health benefits increase with increasing levels of PA, especially when this activity is primarily aerobic in nature. ${ }^{1}$ Finally, establishing proper PA habits at an early age can help children and youth maintain high levels of PA as they transition through adolescence into adulthood, 
contributing to the prevention of a wide range of co-morbidities (e.g. type 2 diabetes mellitus, cardiovascular disease) later in life. ${ }^{43,44}$

Operationally, guidelines can be prescribed with help from the FITT principle (i.e., Frequency, Intensity, Time, and Type). ${ }^{13}$ Frequency refers to how often an individual should be engaging in the activity. For children and youth, it is recommended that they accumulate 60 minutes every day. The intensity of PA is important and refers to the rate at which the activity is being performed and the relative magnitude of effort required. It appears that activities of at least moderate intensity are most beneficial for health. In children and youth, MVPA has been defined in a variety of ways, and using many different cut-points. ${ }^{1}$ The lower threshold for moderate-intensity is most commonly defined as 4 METs (or 4 times their resting metabolic rate), and the most common threshold for vigorous-intensity activity is defined as 7 METs (or 7 times their resting metabolic rate). ${ }^{1}$ Time refers to the duration of activity; for children, it is suggested that they accumulate at least 60 minutes per day, and this can be done in bouts of any length to account for the sporadic nature in which children move and play. Finally, type refers to what kind of activity, such as strength training, aerobic, or balance and flexibility. ${ }^{13}$ 


\section{Canadian Physical Activity Guidelines}

FOR CHILDREN - 5 - 11 YEARS

\section{Guidelines}

For health benefits, children aged 5-11 years should accumulate at least 60 minutes of moderate- to vigorous-intensity physical activity daily. This should include:

Vigorous-intensity activities at least 3 days per week.

Activities that strengthen muscle and bone at least 3 days per week.

More daily physical activity provides greater health benefits.

Let's Talk Intensity!

Moderate-intensity physical activities will cause children to sweat a little and to breathe harder. Activities like:

- Bike riding

- Playground activities

Vigorous-intensity physical activities will cause children to sweat and be 'out of breath'. Activities like:

- Running

- Swimming
Being active for at least $\mathbf{6 0}$ minutes daily can help children:

- Improve their health

- Do better in school

- Improve their fitness

- Grow stronger

- Have fun playing with friends

- Feel happier

- Maintain a healthy body weight

- Improve their self-confidence

- Learn new skills

Parents and caregivers can help to plan their child's daily activity. Kids can:

$\square$ Play tag - or freeze-tag!

$\square$ Go to the playground after school.

$\square$ Walk, bike, rollerblade or skateboard to school. $\square$ Play an active game at recess.

$\square$ Go sledding in the park on the weekend.

$\square$ Go "puddle hopping" on a rainy day.
60 minutes a day. You can
help your child get there!

Figure 2.4. Canadian Physical Activity Guidelines for Children (aged 5-11 years) ${ }^{14}$
CSEP|SCPE

wwow.csep.ca/guidelines 


\subsubsection{Sedentary behaviour guidelines}

In addition to the development of new PA guidelines for children and youth, the Canadian Society for Exercise Physiology, in partnership with the Healthy Active Living and Obesity Research Group and ParticipACTION, released the Canadian SB Guidelines for Children (aged 5-11 years) and Youth (aged 12-17 years) in 2011. ${ }^{17}$

Canadian Sedentary Behaviour Guidelines for Children (aged 5-11 years) and Youth (aged 12-17 years) state that children and youth should minimize time spent being sedentary, including limiting ST to no more than two hours per day, and limiting passive transport, prolonged sitting, and time spent indoors (Figure 2.3). ${ }^{17}$ Operationally, meeting SB guidelines typically refers to children and youth who accumulate no more than two hours of recreational screen time per day. Similarly to the FITT principle to describe PA prescription, a SITT principle (i.e., Sedentary behaviour, Interruptions, Time, and Type) has been suggested to describe SB. ${ }^{15}$ SB refers to the frequency, or number of bouts of sedentary time over a defined time period. Interruptions refers to when sedentary time is broken up by being physically active. ${ }^{45}$ Time refers to the time, or duration of SB. And type refers to the mode of SB (e.g., reading or watching $\mathrm{TV}$ ). 


\section{Canadian Sedentary Behaviour Guidelines}

FOR CHILDREN - 5 - 11 YEARS

\section{Guidelines}

For health benefits, children aged $5-11$ years should minimize the time they spend being sedentary each day. This may be achieved by

Limiting recreational screen time to no more than 2 hours per day; lower levels are associated with additional health benefits.

Limiting sedentary (motorized) transport, extended sitting and time spent indoors throughout the day.

The lowdown on the slowdown: what counts as being sedentary?

Sedentary behaviour is time when children are doing very little physical movement. Some examples are:

- Sitting for long periods

- Using motorized transportation (such as a bus or a car)

- Watching television

- Playing passive video games

- Playing on the computer
Spending less time being sedentary can help children:

- Maintain a healthy body weight

- Do better in school

- Improve their self-confidence

- Have more fun with their friends

- Improve their fitness

- Have more time to learn new skills

Cutting down on sitting down. Help children swap sedentary time with active time!

Wake Up Drive to School
$\begin{aligned} & \text { Active Transpertation } \\ & \text { Instead of driwing walk to } \\ & \text { school with a group of kids } \\ & \text { from the neighbourtood. }\end{aligned}$

There is no time like right now for children to get up and get moving!

Figure 2.5. Canadian Sedentary Behaviour Guidelines for Children (aged 5-11 years) ${ }^{17}$ 


\subsection{Awareness of current Canadian guidelines}

Dissemination strategies for the current PA and SB guidelines followed a similar plan to that of previous guidelines with an initial media release followed by promotion through researchers, health care providers, governmental and non-governmental organizations, teachers, and those with an interest in healthy active living. To date, the primary dissemination channel for Canadian guidelines has been through mass media campaigns including print, television and radio campaigns, and websites. Previous work has shown low awareness of PA guidelines in Canada and elsewhere ${ }^{47-49}$ but since the new Canadian guidelines were released in 2011 and 2012, little work has been done to gauge awareness or uptake. Therefore, part of the background work to inform this thesis was to conduct a comprehensive review of knowledge and awareness of current guidelines.

Background Manuscript 1 (Appendix B) showed that unprompted awareness of the guidelines, at about $4 \%$, has remained relatively unchanged from awareness of the previous guidelines. This suggests that thus far, dissemination of the guidelines has not been adequate to penetrate awareness within the general public. More encouragingly, among health-related organizations, awareness of Canadian PA guidelines is high $(80.7 \%) .{ }^{50}$ Future work should aim to understand how organizations use the guidelines and seek ways that these organizations can help with awareness and knowledge levels in the general population. These organizations should also be recruited to help disseminate guideline information to their respective networks. 
Overall, awareness of the PA guidelines was higher than awareness of the SB guidelines. Low levels of awareness of the SB guidelines may be in part due to the terminology used. Although people may be familiar with the concept of screen time (i.e., television, video game, or computer use), they may not be familiar with the concept of SB as a distinct health risk factor. ${ }^{15}$ Furthermore, influencing decisions to adopt a new behaviour (e.g., reduce SB) may require different promotion and messaging strategies than influencing the decision to discard an old innovation (e.g., continue to follow the old PA guides). It may also be true that awareness of the PA guidelines is higher simply because they have been around for a longer time, and people have had more time to become familiar with them.

Overcoming low awareness of the guidelines will require collaboration and partnerships with those from public health, health promotion, and academic sectors with specific strategies to promote a "whole day" approach to lifestyle behaviours (i.e., the need to meet both PA and SB guidelines). However, it is important to remember that PA guidelines and messages alone are unlikely to curb the epidemic of inactivity. After becoming aware of the guidelines, individuals still need to have an intention to be active, short-term behaviour changes through increased PA and decreased SB, and long term success in maintaining healthy lifestyle behaviours. Detailed population health advocacy and intervention programs with sustained support are likely required for this to occur.

Most work that has been done to assess awareness or uptake of the guidelines has focused on the guidelines for the early years, children, and youth, with little work 
focusing on the guidelines for adults or older adults. To augment the potential impact of future updates to the guidelines, detailed dissemination and promotion strategies with sustained support are required and efforts should be made to assess and monitor awareness and uptake across all age (and potentially gender) subgroups. To maximize efforts, dissemination should be conducted through intersectoral partnership between federal and provincial governments as well as non-governmental and not-for-profit groups.

\subsection{Conceptual framework}

This research was largely informed by two assumptions: 1) that children's habitual behaviours are a result of multiple competing and interacting influences; and 2) public health messages to improve habitual behaviours are only beneficial if they are known to the general public (including, but not limited to the children, parents, caregivers, and health care providers). Both of these assumptions suggest that children are not completely autonomous and rely on interactions and information from outside sources (e.g., their parents, their school environment) for healthy growth and development. For this reason, an ecological framework was used to help guide my research plan and analysis.

\subsubsection{Ecological framework}

Ecological models are believed to provide comprehensive frameworks for understanding multiple interactions and influences of behaviour. Early work to explain the ecological framework used a 'systems theory' approach and focused on three levels of environmental influences: 1) the microsystem includes interactions among work 
groups and family members; 2) the mesosystem includes the physical family, school and work setting; and 3) the exosystem, which includes the larger social system of culture, politics and economics. ${ }^{51}$ Krieger has expanded upon this to include other aspects of social behaviour and eliminate the idea of proximal versus distal influences. ${ }^{52}$ She suggested the need to understand the origins of population risk factors and their implications for population health. That group patterns are not simply the sum of individual traits and choices, but influenced by varying degrees at different levels. ${ }^{53}$ Further, a central thought to all ecological models is that multi-faceted, multi-level interventions are needed to support sustained behaviour change. ${ }^{54}$

Ecological models have often been adapted to help understand public health research. Specifically, the ecological model has been used frequently by public health researchers to help explain the relationship between intrapersonal (biological, psychological), interpersonal (social, cultural), organizational, community, physical environment, and policy factors. ${ }^{55}$ Because of the influence of many factors in the broader social context, ecological approaches have become prominent in PA and SB research. ${ }^{56-58}$ Sallis et al. ${ }^{57}$ has proposed a model for health behaviour, specifically for PA (Figure 2.6). This work is highlighted here to provide the reader with some historical context. Although the focus of this dissertation is on SB, the work to develop an ecological model for active living by Sallis et al. has been key in advancing health behaviour research and was used to inform a proposed ecological model for SB. ${ }^{56}$ The ecological model proposed by Owen et al. describing SB is outlined in detail below and shown in Figure 2.7. ${ }^{56}$ 


\subsubsection{Determinants of active living and sedentary behaviour}

Early research examining correlates of active living were limited to those in the fields of physical education, physiology, and health, and were often limited by small sample sizes, modest effect sizes, and poor long-term follow-up. ${ }^{57}$ As the field of active living expanded to include other disciplines (e.g., epidemiology, urban planning), so did our understanding that the associations between lifestyle habits and health outcomes were complex. Therefore, recent work has suggested the need for multilevel interventions targeting all levels and the interactions between individuals, social environments, physical environments, and policies. ${ }^{57}$ Sallis and colleagues used findings and concepts from the fields of health, behavioural science, transportation and city planning, policy studies and economics, and leisure sciences to create a model to help understand the influences of PA. ${ }^{57}$

In his work to develop an ecological model of health behaviour, Sallis et al. ${ }^{57}$ proposed four core principles: “1) that there are multiple levels of influence on specific health behaviors; 2 ) that influences on behaviours interact across these different levels; 3) that ecological models should be behaviour-specific; and 4) that multi-level interventions are most effective in changing behaviour". ${ }^{57}$

Sallis et al. ${ }^{57}$ suggest that the overarching domains of PA include leisure time, active transport, household activities, and occupation (or school) activities. Sallis developed the domains with adults in mind, but they may be applied to all age groups, although interventions or policy variables are likely distinct to smaller subgroups like children and youth. For example, leisure time, especially the after school period, is 
thought to be a critical time to increase PA in children and youth. ${ }^{59}$ Active transportation, defined as walking, biking, in-line skating, and skateboarding to get to and from places such as school, parks and shops, has been shown to be an important contributor to increase habitual PA. ${ }^{60}$ Household activities, such as chores, and family based PA are important teachable moments to help children develop good activity habits for the future. School time, via partnerships with teachers, school officials, and policy makers is seen as good time to take advantage of a captive audience and encourage increases in positive knowledge, attitudes, and beliefs surrounding PA. It is also important to remember that children and youth are not (for the most part) autonomous in their behaviours and are also influenced by their parents, teachers, and caregivers. The complex nature of childhood growth and development provides added confirmation that ecological models are needed to describe their behaviour.

As mentioned, a similar model has been developed to understand determinants of SB. Owen et al. ${ }^{56}$ describe domains of SB to include occupation (or school), transportation, leisure time, and household time. Of particular importance is the behavioural setting and associated social contexts where sitting occurs. Some SB is of course inevitable throughout the day (e.g., family dinners, going to school, doing homework), but it is important to understand factors that are related to high, or prolonged bouts of SB.

The model to describe domains of SB applies across most age groups but it is important to remember that children are often not autonomous in their choices and rely on parents, caregivers, and teachers to provide them with opportunities to 
decrease their SED. Children also lack the mental capacity to fully understand the deleterious and long-term health effects associated with prolonged bouts of SB. The concept of autonomy and cognitive understanding may be more important when examining SB, since most children (and adults) enjoy activities like watching TV or playing video games it may be harder for parents to intervene. Parents may also have their own motivations for letting their children engage in ST as a means of babysitting while they are doing chores. The model for SB may also need to be adapted for different demographics. ${ }^{56}$ For example, those in low-income or developing countries may not have access to the same types of screen-based technology as those in developed or high-income countries. Or, with programs like Netflix, watching TV may be considered the same activity as playing on the computer since they can be both done on a laptop. 


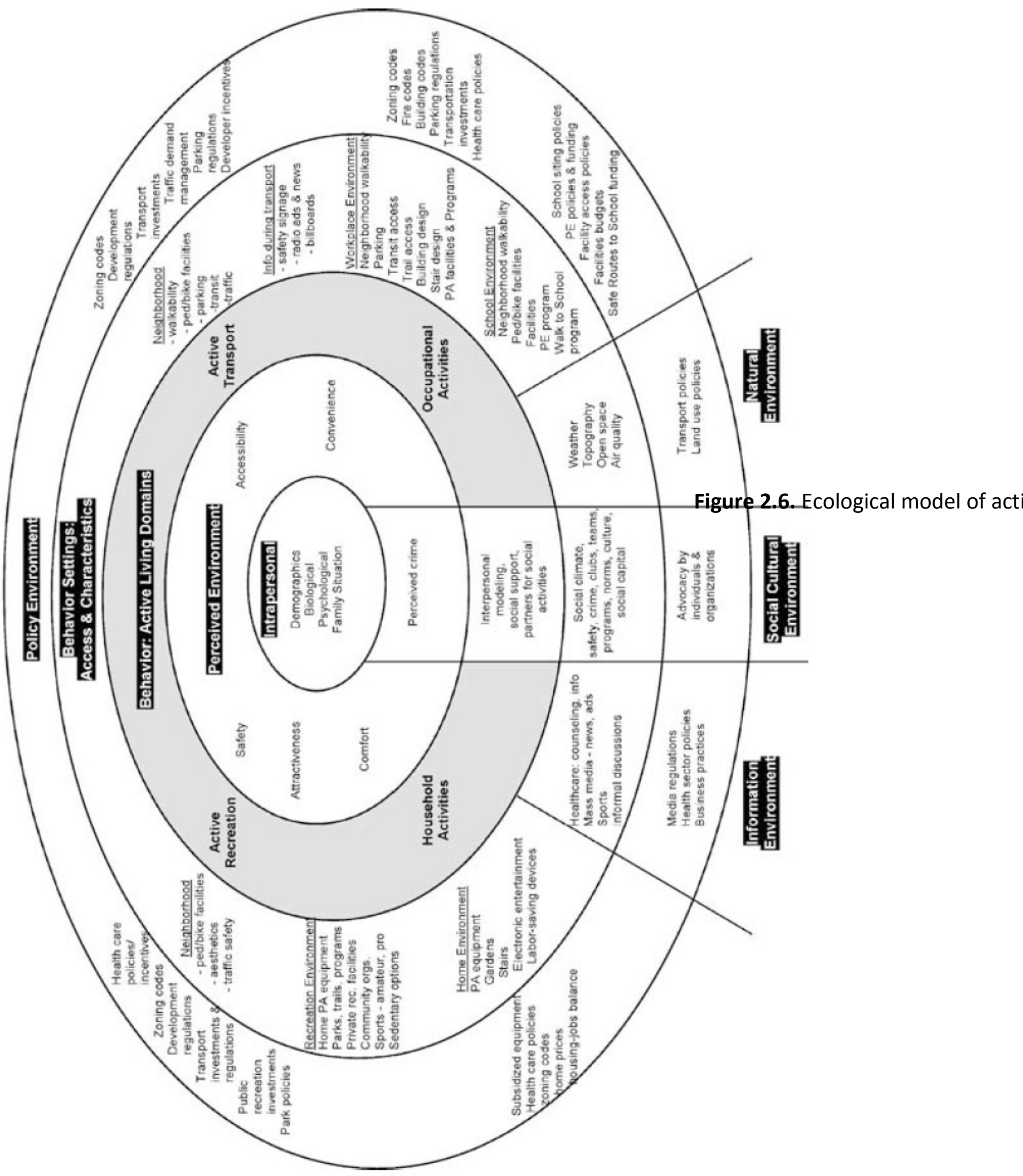




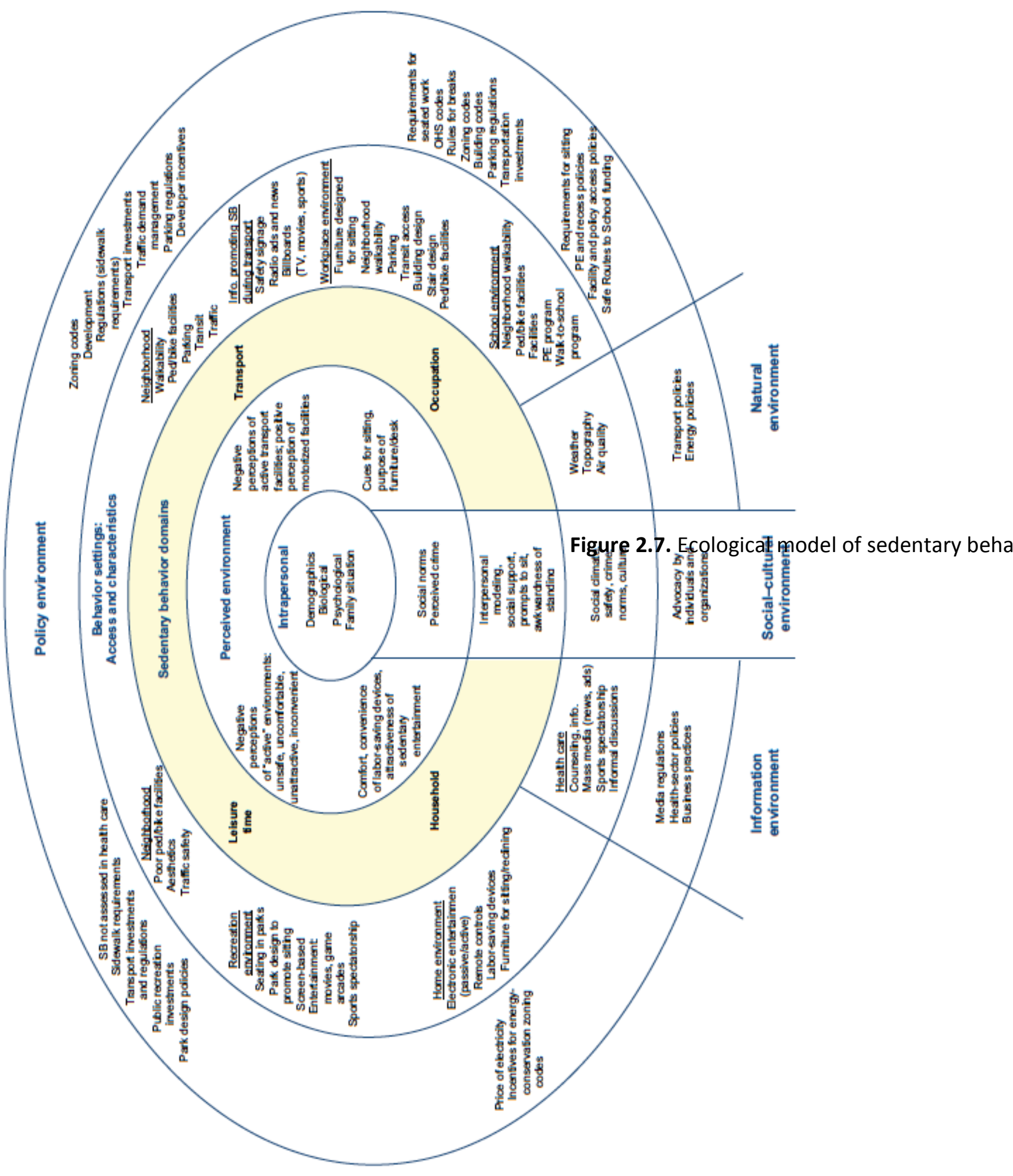




\subsection{Importance of studying sedentary behaviour in a population health context}

There have been hundreds of studies examining PA and SB in children and youth. However, with a great diversity in research designs, measurement approaches, theories used, and variables tested, it becomes difficult to integrate the findings and update the status of the knowledge in the field.$^{61}$ This reality limits the ability of subsequent research to build on previous findings. It is particularly difficult to integrate knowledge from cross-sectional studies since no comment can be made on the direction of causality. ${ }^{62}$

In medical research (e.g., disease pathology), the causal pathway is often quite clear; once a virus is treated, the illness dissipates. However, there are no, or very few, cases in behavioural research where the causal factors "cause" the outcome in $100 \%$ of the cases. This is further complicated by the possibility of multiple causal factors influencing a particular behaviour, or with bi-directional relationships affecting both the exposure and the outcome.

It is also possible that deliberate population health interventions do not have any effect on a behaviour, or that changes in a behaviour are due to a change in an unrelated shift in policy or natural environment that were not expected to have an effect. Exposure to a factor does not necessarily lead to a change in the behavioural outcome. This also means that causal pathways, and methods for measuring them in population health research is complex and less clear. ${ }^{63}$

The misinterpretation of results from a cross sectional study can be minimized through analytical techniques, and by properly accounting for factors that may 
intervene on the causal pathway between dependent and independent variables (e.g., mediators, confounders). Analytical techniques used throughout this work are discussed in great detail in Chapter 3. In brief, this work will use primarily multilevel modeling technique to properly account for the hierarchical nature of the data collected in ISCOLE. This allows us to examine the relevant distributions of health outcomes and the distribution of variance in health outcomes that can be attributed to each level that has been included in the model.

Factors that may intervene on the causal pathway generally include mediators, moderators, and confounders. An in depth discussion of these factors is beyond the scope of this work, but brief definitions are provided as they are important to consider in any epidemiological research. Mediators are factors that are on the causal pathway between the dependent and independent variables (e.g. outside factors that also affect the outcome of interest). There may be a single mediator, or a series of cascading mediators to take into account. Confounders are variables that help to predict an outcome, but are also associated with the exposure thereby distorting the true magnitude of effect. There are likely many potential confounders to consider when building a model (e.g., age, ethnicity). Moderators affect the strength of the relationship between an exposure and an outcome. This is analogous to interaction terms and requires analysis to be stratified by the potential moderator (e.g., stratifying the analysis by age group or sex). Bauman et al. ${ }^{62}$ has provided an excellent overview of the role of determinants, correlates, causal variables, mediators, moderators, and confounders and provides a useful schematic. This has been adapted in Figure 2.7. As mentioned, 
although this work will not focus specifically on determining mediators or moderators of SB, these terms are useful to properly understand when trying to interpret results from epidemiological studies.

Mediator variables (M)

Confounder (B)

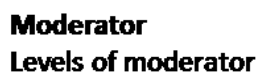

Levels of moderator
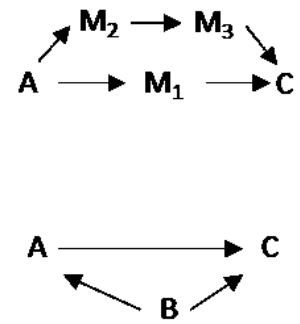

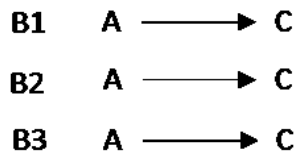

Figure 2.8. Description of mediators, confounders, and moderators ${ }^{62}$

\subsubsection{Correlates of sedentary behaviour in a population health context}

Correlates of behaviour should not generally be thought of as causal factors, but rather as possible predictors of differences in behaviours across groups. ${ }^{62}$ Practically, studying correlates of behaviour provides evidence to help design a behavioural intervention study; theoretically, correlational studies can test the assumptions made in various theories or models, which can then produce results to strengthen, or modify these assumptions.$^{62}$ Cross- sectional studies provide an efficient means to test many correlates at the same time. A recent review by Temmel and Rhodes ${ }^{64}$ provided us with the most comprehensive list of studies examining correlates of objectively measured SED and self-, or parent-reported ST. They identified 64 studies examining correlates of SB; however, only eleven reported on both accelerometer- measured SED and self- 
reported ST in the same population. ${ }^{64}$ Studies included participants from around the world (although predominantly the United States), but none of the 64 studies included data from Canadian children. Of the eleven studies examining both SED and ST, age, sex, ethnicity, socio-economic status, and day of the week (weekend versus weekday) were significant correlates of both SED and ST. Physical maturity was a correlate of SED only; whereas urban versus rural living, neighborhood satisfaction and safety, access (e.g., to a television), sleep duration, and self-esteem were correlates of ST only. Other studies that have explored associations with ST have identified age, socio-economic status, single-parent households, ethnicity, and sex as important correlates. ${ }^{65,66}$ Studies that have examined correlates of SED have shown significant associations with parental BMI, TV viewing, and computer use. ${ }^{67}$ The work presented in this dissertation will aim to first understand similarities and differences in correlates of SED and ST in a sample of Canadian children, and then expand upon this by examining correlates in participants from the 12 ISCOLE study sites. It is hoped that this may help to inform future population health interventions to reduce SED and ST, and potentially be used to inform more specific messaging related to Canadian SB guidelines. 


\section{References}

1. Janssen, I. \& LeBlanc, A. G. Systematic review of the health benefits of physical activity and fitness in school-aged children and youth. Int. J. Behav. Nutr. Phys. Act. 7, 40 (2010).

2. Paterson, D. \& Warburton, D. Physical Activity and Functional Limitations in Older Adults: A Systematic Review Related to Canada's Physical Activity Guidelines. Int. J. Behav. Nutr. Phys. Act. 7, (2010).

3. Warburton, D. E., Charlesworth, S., Ivey, A., Nettlefold, L. \& Bredin, S. S. A systematic review of the evidence for Canada's Physical Activity Guidelines for Adults. Int. J. Behav. Nutr. Phys. Act. 7, 39 (2010).

4. Colley, R. C. et al. Physical activity of Canadian children and youth: Accelerometer results from the 2007 to 2009 Canadian Health Measures Survey. Health Rep. 22, 15-23 (2011).

5. Colley, R. C. et al. Physical activity of Canadian adults: accelerometer results from the 2007 to 2009 Canadian Health Measures Survey. Health Rep. Stat. Can. Can. Cent. Health Inf. Rapp. Sur Santé Stat. Can. Cent. Can. Inf. Sur Santé 22, 7-14 (2011).

6. Pate, R. R., Mitchell, J. A., Byun, W. \& Dowda, M. Sedentary behaviour in youth. Br. J. Sports Med. 45, 906-913 (2011).

7. Olshansky, S. J. et al. A Potential Decline in Life Expectancy in the United States in the 21st Century. N. Engl. J. Med. 352, 1138-1145 (2005).

8. Kindig, D. A. Understanding Population Health Terminology. Milbank Q. 85, 139161 (2007). 
9. Kindig, D. \& Stoddart, G. What Is Population Health? Am. J. Public Health 93, 380-383 (2003).

10. Public Health Agency of Canada. What is population health? What is the population health approach (2012).

11. Kohl, H. W. et al. The pandemic of physical inactivity: global action for public health. The Lancet 380, 294-305 (2012).

12. Pate, R. \& Pratt, M. Physical activity and public health: A recommendation from the Centers for Disease Control and Prevention and the American College of Sports Medicine. JAMA 273, 402-407 (1995).

13. Oberg, E. Physical Activity Prescription: Our Best Medicine. Integr. Med. 6, 18-22 (2007).

14. Tremblay, M. S. et al. New Canadian Physical Activity Guidelines. Appl. Physiol. Nutr. Metab. 36, 36-46 (2011).

15. Tremblay, M. S., Colley, R. C., Saunders, T. J., Healy, G. N. \& Owen, N. Physiological and health implications of a sedentary lifestyle. Appl. Physiol. Nutr. Metab. 35, 725-740 (2010).

16. Sedentary Behaviour Research Network. Letter to the Editor: Standardized use of the terms 'sedentary' and 'sedentary behaviours'. Appl. Physiol. Nutr. Metab. 37, 540542 (2012).

17. Tremblay, M. S. et al. Canadian Sedentary Behaviour Guidelines for Children and Youth. Appl. Physiol. Nutr. Metab. 36, 59-64 (2011). 
18. Evenson, K. R., Catellier, D. J., Gill, K., Ondrak, K. S. \& McMurray, R. G. Calibration of two objective measures of physical activity for children. J. Sports Sci. 26, 1557-1565 (2008).

19. Physical Activity Advisory Committee. Physical activity guidelines advisory committee. (U.S. Department of Health and Human Services, 2008).

20. Bull, F. C. \& Expert Working Group. Physical activity guidelines in the U.K.: review and recommendations. (School of Sport, Exercise, and Health Sciences, Loughborough University, 2010).

21. Okely, A. D., Salmon, J., Trost, S. G. \& Hinkley, T. Discussion paper for the development of physical activity recommendations for children under 5 years. (Australian Government Department of Health and Ageing, 2008).

22. World Health Organization. Global recommendations on physical activity for health. (2010). at <http://www.who.int/dietphysicalactivity/publications/9789241599979/en/index.html> 23. Hallal, P. C. et al. Global physical activity levels: surveillance progress, pitfalls, and prospects. The Lancet380, 247-257 (2012).

24. Colley, R. C., Janssen, I. \& Tremblay, M. S. Daily Step Target to Measure Adherence to Physical Activity Guidelines in Children. Med. Sci. Sports Exerc. 44, 977982. (2012).

25. Tammelin, T., Laine, K. \& Turpeinen, S. Oppilaiden fyysinen aktiivisuus. [Physical Activity of School-aged children]. (Liikunnan ja kansanterveyden edistämissäätiö LIKES [Sport and Health LIKES], 2013). 
26. Griffiths, L. J. et al. How active are our children? Findings from the Millennium Cohort Study. BMJ Open 3, e002893 (2013).

27. Tudor-Locke, C., Johnson, W. D. \& Katzmarzyk, P. T. Accelerometer-Determined Steps per Day in US Children and Youth: Med. Sci. Sports Exerc. 42, 2244-2250 (2010).

28. Department of Health and Ageing. Australian National Children's Nutrition and Physical Activity Survey (ANCNPAS): main findings. (Commonwealth Scientific and Industrial Research Organisation (CSIRO) and the University of South Australia, 2008).

29. Currie, C. et al.Inequalities in Young People's Health: Health Behaviour in Schoolaged Children. International Report from the 20052006 Survey Health Policy for Children and Adolescents. (2008).

30. Statistics South Africa. A time of use. (Statistics South Africa, 2013).

31. Cao, H. et al. Screen time, physical activity and mental health among urban adolescents in China. Prev. Med. 53, 316-320 (2011).

32. Australian Bureau of Statistics. Australian Health Survey: Physical Activity, 201112. (2013). at <http://www.abs.gov.au/ausstats/abs@.nsf/Lookup/4364.0.55.001main+features12011 $-12>$

33. Healy, G. N., Matthews, C. E., Dunstan, D. W., Winkler, E. A. H. \& Owen, N. Sedentary time and cardio-metabolic biomarkers in US adults: NHANES 2003-06. Eur. Heart J. 32, 590-597 (2011). 
34. Proper, K. I., Singh, A. S., van Mechelen, W. \& Chinapaw, M. J. M. Sedentary Behaviors and Health Outcomes Among Adults: A Systematic Review of Prospective Studies. Am. J. Prev. Med. 40, 174-182 (2011).

35. Tremblay, M. S. et al. Systematic review of sedentary behaviour and health indicators in school-aged children and youth. Int. J. Behav. Nutr. Phys. Act. 8, 98 (2011). 36. Saunders, T. J. et al. Prolonged sitting and markers of cardiometabolic disease risk in children and youth: a randomized crossover study. Metabolism. 62, 1423-1428 (2013).

37. Colley, R. C. et al. The association between accelerometer-measured patterns of sedentary time and health risk in children and youth: results from the Canadian Health Measures Survey. BMC Public Health 13, 200 (2013).

38. Grimshaw, J. \& Russell, I. Achieving health gain through clinical guidelines. I: Developing scientifically valid guidelines. Qual. Health Care 2, 243-248 (1993).

39. Tremblay, M. S. et al. Canadian Physical Activity Guidelines for the Early Years (aged 0-4 years). Appl. Physiol. Nutr. Metab. 37, 345-356 (2012).

40. Timmons, B. W. et al. Systematic review of physical activity and health in the early years (aged 0-4 years). Appl. Physiol. Nutr. Metab. 37, 773-792 (2012).

41. Tremblay, M. S. et al. Canadian Sedentary Behaviour Guidelines for the Early Years (aged 0-4 years). Appl. Physiol. Nutr. Metab. Physiol. Appliquée Nutr. Métabolisme 37, 370-391 (2012). 
42. LeBlanc, A. G. et al. Systematic review of sedentary behaviour and health indicators in the early years (aged 0-4 years). Appl. Physiol. Nutr. Metab. 37, 753-772 (2012).

43. Janssen, I. et al. Utility of Childhood BMI in the Prediction of Adulthood Disease: Comparison of National and International References. Obes. Res. 13, 1106-1115 (2005).

44. Ruiz, J. R. et al. Predictive validity of health-related fitness in youth: a systematic review. Br. J. Sports Med. 43, 909-923 (2009).

45. Healy, G. N. et al. Breaks in Sedentary Time Beneficial associations with metabolic risk. Diabetes Care 31, 661-666 (2008).

46. Tremblay, M. S., Shephard, R. J. \& Brawley, L. R. Research that informs Canada's physical activity guides: an introduction. Appl. Physiol. Nutr. Metab. 32, S1-S8 (2007).

47. Spence, J. C., Plotnikoff, R. C. \& Mummery, W. K. The awareness and use of Canada's Physical Activity Guide to Healthy Active Living. Can. J. Public Health Rev. Can. Santé Publique 93, 394-396 (2002).

48. Berry, T. R., Witcher, C., Holt, N. L. \& Plotnikoff, R. C. A Qualitative Examination of Perceptions of Physical Activity Guidelines and Preferences for Format. Health Promot. Pract. 11, 908-916 (2010).

49. Debastiani, S. D., Carroll, D. D., Cunningham, M., Lee, S. \& Fulton, J. Awareness and Knowledge of the Youth 2008 Physical Activity Guidelines for Americans. J. Phys. Act. Health 11, 495-501 (2013). 
50. Gainforth, H. L. et al. Evaluating the uptake of Canada's new physical activity and sedentary behaviour guidelines on service organizations' websites. Transl. Behav. Med. 3, 172-179 (2013).

51. Bronfenbrenner, U. The ecology of human development: Experiments by nature and design. (Harvard University Press, 1979).

52. Krieger, N. Epidemiology and the web of causation: Has anyone seen the spider? Soc. Sci. Med. 39, 887-903 (1994).

53. Krieger, N. Proximal, Distal, and the Politics of Causation: What's Level Got to Do With It? Am. J. Public Health 98, 221-230 (2008).

54. Sallis, J. F., Owen, N. \& Fisher, E. B. in Health behavior and health education: Theory, research, and practice (4th ed.) (eds. Glanz, K., Rimer, B. K. \& Viswanath, K.) 465-485 (Jossey-Bass, 2008).

55. Stokols, D. Establishing and maintaining healthy environments: Toward a social ecology of health promotion. Am. Psychol.47, 6-22 (1992).

56. Owen, N. et al. Adults' Sedentary Behavior: Determinants and Interventions. Am. J. Prev. Med. 41, 189-196 (2011).

57. Sallis, J. F. et al. An Ecological Approach to Creating Active Living Communities. Annu. Rev. Public Health 27, 297-322 (2006).

58. Spence, J. C. \& Lee, R. E. Toward a comprehensive model of physical activity. Psychol. Sport Exerc. 4, 7-24 (2003).

59. Active Healthy Kids Canada. Don't let this be the most physical activiyt our kids get after school. (Active Health Kids Canada, 2011). at <www.activehealthykids.ca> 
60. Pabayo, R. et al. The importance of Active Transportation to and from school for daily physical activity among children. Prev. Med. 55, 196-200 (2012).

61. Bauman, A. E., Nelson, D. E., Pratt, M., Matsudo, V. \& Schoeppe, S. Dissemination of Physical Activity Evidence, Programs, Policies, and Surveillance in the International Public Health Arena. Am. J. Prev. Med. 31, 57-65 (2006).

62. Bauman, A. E., Sallis, J. F., Dzewaltowski, D. A. \& Owen, N. Toward a better understanding of the influences on physical activity. Am. J. Prev. Med. 23, 5-14 (2002).

63. Maziak, W. Is uncertainty in complex disease epidemiology resolvable? Emerg. Themes Epidemiol. 12, 7 (2015).

64. Temmel, C. S. D. \& Rhodes, R. Correlates of Sedentary Behaviour in Children and Adolescents Aged 7-18: A Systematic Review. Health Fit. J. Can. 6, 119-199 (2013).

65. Brodersen, N. H., Steptoe, A., Boniface, D. R. \& Wardle, J. Trends in physical activity and sedentary behaviour in adolescence: ethnic and socioeconomic differences. Br. J. Sports Med. 41, 140-144 (2007).

66. Van Der Horst, K., Chin A Paw, M. J., Twisk, J. W. R. \& Van Mechelen, W. A brief review on correlates of physical activity and sedentariness in youth. Med. Sci. Sports Exerc. 39, 1241-1250 (2007).

67. Sluijs, E. M. F. van, Page, A., Ommundsen, Y. \& Griffin, S. J. Behavioural and social correlates of sedentary time in young people. Br. J. Sports Med. 44, 747-755 (2010). 


\section{Chapter 3}

\section{Methodological Overview}

\subsection{Summary}

This chapter presents a methodological overview, which aims to help the reader understand the planning (from conception to analysis) that went into each manuscript. Manuscripts 1-3 are presented in Chapter 4 and Background Manuscripts 1 and 2 are presented in the Appendix A and B respectively.

All manuscripts included in Chapter 4 used data from ISCOLE; Manuscript 1 also included data from the Canadian Assessment of Physical Literacy (CAPL). As part of my doctoral training, I assisted with all areas of data collection for ISCOLE Canada and CAPL. Data collection for ISCOLE occurred from October 2011 until December 2013, with data collection for the Canadian site occurring from fall 2012 until spring 2013. CAPL data collection occurred from winter 2010 until spring 2013 with ISCOLE CAPL data collection occurring in spring 2013. Involvement in data collection provided me with excellent insight and thorough knowledge of the dataset before planning my statistical analysis. Research Ethics Board approval was obtained from the Children's Hospital of Eastern Ontario and the University of Ottawa (Appendix C). Child assent and parent consent were also obtained for all aspects of data collection (Appendix C).

\subsubsection{Project framework}

Described in detail below, the work for this doctoral project was informed primarily by data collected in the ISCOLE. This work focuses on influences of SED and ST, 
but information relating to other lifestyle and demographic information has been integrated where appropriate.

\subsection{Summary of research methods and datasets}

\subsubsection{International Study of Childhood Obesity, Lifestyle and the Environment}

The primary aim of ISCOLE was to determine the relationships between lifestyle behaviours and obesity in a multi-national study of 10 -year-old children. ${ }^{1}$ Further, ISCOLE aimed to investigate the influence of higher-order characteristics such as behavioural settings, and the physical, social and policy environments, on the observed relationships within and between countries. ${ }^{1}$ Data were collected on children from five major geographic regions of the world (Europe, Africa, the Americas, Asia, and the Western Pacific) across 12 countries (Australia, Brazil, Canada, China, Colombia, Finland, India, Kenya, Portugal, South Africa, United Kingdom, and United States) with a goal of recruiting at least 500 participants, aged 9-11 years, from each study site. Data collection sites are shown in Figure 3.1 and details on ISCOLE site and participant characteristics can be found in Table 3.1. 


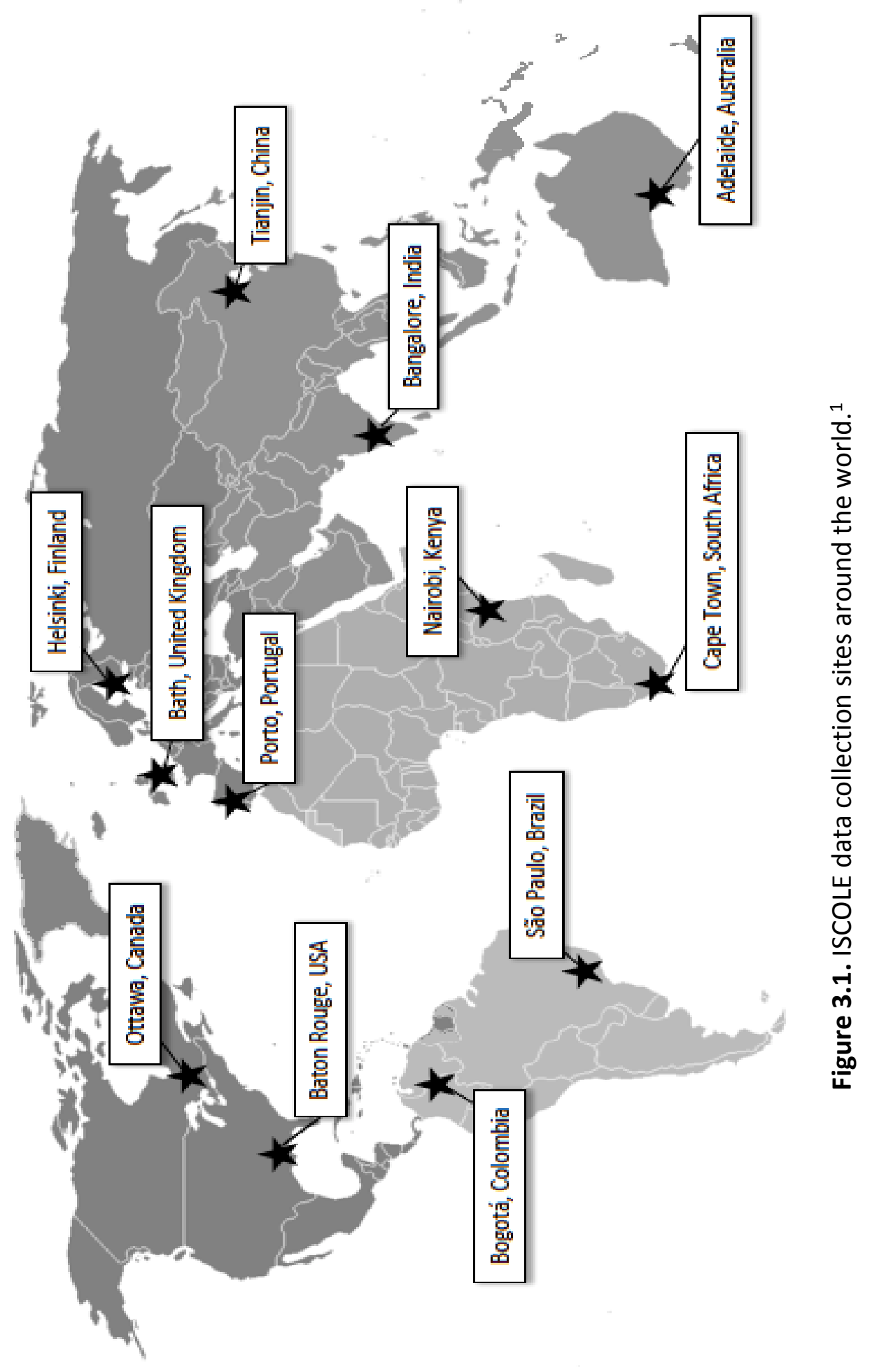


Table 3.1. ISCOLE country and participant characteristics.

\begin{tabular}{|c|c|c|c|c|c|c|}
\hline Country & $\begin{array}{l}\text { National } \\
\text { population }\end{array}$ & $\begin{array}{l}\text { ISCOLE site } \\
\text { location }\end{array}$ & $\begin{array}{l}\text { Population of ISCOLE site } \\
\text { location }^{\text {b }}\end{array}$ & $\begin{array}{l}\text { Participants } \\
\text { ( } n, \% \text { boys) }\end{array}$ & $\begin{array}{l}\text { Age (years) } \\
\text { (mean, SD) }\end{array}$ & Weight status (\%) ${ }^{c}$ \\
\hline \multicolumn{7}{|l|}{ Europe } \\
\hline Finland & $5,442,322$ & $\begin{array}{l}\text { Helsinki, Espoo, } \\
\text { Vantaa }\end{array}$ & $1,060,701$ & $536(47.2 \%)$ & $10.0(0.4)$ & $\begin{array}{l}\text { Normal weight: } 74.6 \\
\text { Overweight/obese: } 23.7\end{array}$ \\
\hline United Kingdom & $63,705,000$ & $\begin{array}{l}\text { Bath, North East } \\
\text { Somerset }\end{array}$ & 177,700 & $525(45.1 \%)$ & $10.4(0.5)$ & $\begin{array}{l}\text { Normal weight: } 68.5 \\
\text { Overweight/obese: } 30.3\end{array}$ \\
\hline Portugal & $10,562,178$ & Porto & 237,584 & 777 (46.1\%) & $10.0(0.3)$ & $\begin{array}{l}\text { Normal weight: } 52.5 \\
\text { Overweight/obese: } 47.2\end{array}$ \\
\hline \multicolumn{7}{|l|}{ Africa } \\
\hline Kenya & $44,354,000$ & Nairobi & $3,138,369$ & $563(46.5 \%)$ & $9.8(0.7)$ & $\begin{array}{l}\text { Normal weight: } 75.1 \\
\text { Overweight/obese: } 21.1\end{array}$ \\
\hline South Africa & $52,981,991$ & Cape Town & $3,497,097$ & $550(40.1 \%)$ & $9.8(0.7)$ & $\begin{array}{l}\text { Normal weight: } 71.2 \\
\text { Overweight/obese: } 26.4\end{array}$ \\
\hline \multicolumn{7}{|l|}{ The Americas } \\
\hline Canada & $35,158,304$ & Ottawa & 883,391 & $567(42.2 \%)$ & $10.0(0.4)$ & $\begin{array}{l}\text { Normal weight: } 68.7 \\
\text { Overweight/obese: } 30.8\end{array}$ \\
\hline United States & $316,783,000$ & Baton Rouge & 802,484 & $651(43.2 \%)$ & $9.5(0.6)$ & $\begin{array}{l}\text { Normal weight: } 58.4 \\
\text { Overweight/obese: } 41.3\end{array}$ \\
\hline Colombia & $47,262,816$ & Bogotá & $7,674,366$ & $919(49.4 \%)$ & $10.0(0.6)$ & $\begin{array}{l}\text { Normal weight: } 75.7 \\
\text { Overweight/obese: } 22.9\end{array}$ \\
\hline Brazil & $201,032,714$ & $\begin{array}{l}\text { Sao Caetano do } \\
\text { Sul }\end{array}$ & 149,263 & 584 (49.1\%) & $10.1(0.5)$ & $\begin{array}{l}\text { Normal weight: } 52.8 \\
\text { Overweight/obese: } 45.2\end{array}$ \\
\hline \multicolumn{7}{|l|}{ South Asia } \\
\hline India & $1,242,456,566$ & Bangalore & $9,588,910$ & 620 (47.1\%) & $10.0(0.6)$ & $\begin{array}{l}\text { Normal weight: } 61.5 \\
\text { Overweight/obese: } 33.7\end{array}$ \\
\hline \multicolumn{7}{|l|}{ Western Pacific } \\
\hline China & $1,362,620,526$ & Tianjin & $10,290,987$ & 552 (53.1\%) & $9.4(0.5)$ & $\begin{array}{l}\text { Normal weight: } 56.3 \\
\text { Overweight/obese: } 41.2\end{array}$ \\
\hline Australia & $23,235,207$ & Adelaide & $1,212,982$ & $528(46.0 \%)$ & $10.3(0.5)$ & $\begin{array}{l}\text { Normal weight: } 61.4 \\
\text { Overweight/obese: } 37.9\end{array}$ \\
\hline
\end{tabular}

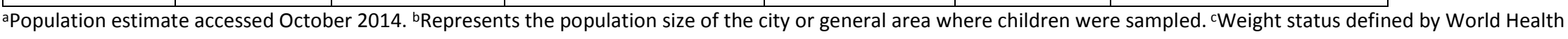
Organization cut-points ${ }^{2}$. 
The first two manuscripts included as part of this dissertation focused on ISCOLE Canada. ISCOLE Canada collected data on 567 children (mean age $=10.0$ years) from the greater Ottawa region in Ottawa, Canada. Manuscript 3 used data from the complete ISCOLE dataset. Overall, data were available on 7,372 children; however, the analytical sample used in each manuscript varied slightly based on participants with missing data. The administrative framework and specific data collection protocols for ISCOLE were clearly set out by the coordinating center at the Pennington Biomedical Research Center, in consultation with each country's Principal Investigator, to ensure a high level of quality control and to minimize measurement variation and bias between study sites. ${ }^{1}$ ISCOLE sites made an effort to stratify their sample by indicators of socioeconomic status to maximize variability, and generally had a goal of including at least 20 schools, with approximately 25-30 children per school (500 children minimum per site). ${ }^{1}$ Many countries included both private and public schools, although all sites limited their data collection to large cities, and urban or suburban schools (i.e., no country collected data from participants living in remote or rural areas, or attending rural schools). See Table 3.2 for additional details on site-specific data collection strategies (Table 3.2). 
Table 3.2. Sampling methods employed by International Study of Childhood Obesity, Lifestyle and the Environment (ISCOLE) sites. Adapted from Katzmarzyk et al. ${ }^{1}$

\begin{tabular}{|c|c|}
\hline ISCOLE Site & Sampling Strategy \\
\hline Australia & $\begin{array}{l}\text { A stratified probability sampling frame was used, aiming to ensure that each } 5^{\text {th }} \text { grade child had equal } \\
\text { chance of being selected. Schools were initially stratified into tertiles based measures of socio- } \\
\text { economic status. Schools were chosen randomly within each tertile until the complete data from the } \\
\text { required number of children ( } 200 \text { children) from each tertile obtained. }\end{array}$ \\
\hline Brazil & $\begin{array}{l}\text { In the region of Sao Caetano do Sul, public schools represent the lower socio-economic strata, and } \\
\text { private schools reflect the higher socio-economic strata. Random lists of public and private elementary } \\
\text { schools in the region were generated, and schools were selected for each list at a ratio of } 4 \text { (public) to } \\
1 \text { (private). Twenty schools ( } 16 \text { public and } 4 \text { private), with } 20-25 \text { students per school were sampled. }\end{array}$ \\
\hline Canada & $\begin{array}{l}\text { Schools were drawn from the Ottawa Region. Schools were stratified into four groups: English Public, } \\
\text { French Public, English Catholic, French Catholic). All schools within each stratum were invited to } \\
\text { participate and the first to respond were included into the study until each stratum was filled. }\end{array}$ \\
\hline China & $\begin{array}{l}\text { Three regions ( } 2 \text { urban districts and } 1 \text { suburban district) of Tianjin city were selected and stratified } \\
\text { according to three levels of socio-economic status. Within each stratum, } 2 \text { schools are selected } \\
\text { randomly from a list of all public schools, with a total of } 6 \text { schools to participate. Each school ensured } \\
\text { an average sample size of } 85-90 \text { students. }\end{array}$ \\
\hline Colombia & $\begin{array}{l}\text { A list of public and private schools in Bogotá were selected according to a list of inclusion criteria } \\
\text { including school geography (i.e., urban), demographic characteristics (e.g., including both boys and } \\
\text { girls), socio-economic status, and school characteristics (e.g., school calendar). Schools were sorted } \\
\text { into high SES, middle SES and low SES and was designed to include at least } 500 \text { children from } 20 \\
\text { schools. }\end{array}$ \\
\hline Finland & $\begin{array}{l}\text { The sampling frame was a complete list of primary schools in the cities of Helsinki, Vantaa and Espoo. } \\
\text { Schools were first stratified by city and then by area SES (high, low) based on socio-economic } \\
\text { characteristics of their geographical location. From each of these } 6 \text { strata (city/SES), three to six } \\
\text { schools were randomly selected to represent the distribution of pupils and SES within the total } \\
\text { sampling area. }\end{array}$ \\
\hline India & $\begin{array}{l}\text { Based on school fee structure, a classification was made into low, middle and high socio-economic } \\
\text { status. Three to four consenting schools were selected from each stratum. The children were sampled } \\
\text { to have at least } 60-70 \text { students from each school. }\end{array}$ \\
\hline Kenya & $\begin{array}{l}\text { Non-boarding primary schools from Nairobi County were stratified into public and private (boarding } \\
\text { schools were not sampled). The schools were then selected proportional to the distribution of public } \\
\text { and private school attendance. }\end{array}$ \\
\hline Portugal & $\begin{array}{l}\text { There is little variability in socio-economic status at the school level in Porto; thus schools were } \\
\text { randomly selected from a list provided by the North Regional Education Directory Board. Twenty } \\
\text { schools were sampled, and from each, } 5^{\text {th }} \text { grade students were sampled in order to have } 25-30 \\
\text { students in each school. }\end{array}$ \\
\hline $\begin{array}{l}\text { South } \\
\text { Africa }\end{array}$ & $\begin{array}{l}\text { Participants were recruited through a list of all public schools within Cape Town. The list was stratified } \\
\text { according to SES quintiles and at least } 4 \text { schools were randomly selected from each stratum for a total } \\
\text { of at least } 20 \text { schools. }\end{array}$ \\
\hline $\begin{array}{l}\text { United } \\
\text { Kingdom }\end{array}$ & $\begin{array}{l}\text { The sampling frame was a complete list of primary schools in the Bath and North East Somerset } \\
\text { regions. Schools were stratified according to size and socio-economic characteristics. From each } \\
\text { stratum, a proportional cluster was selected. Specifically, schools were randomly selected using the } \\
\text { probability proportional to size approach and a reserve-list compiled to account for school withdrawal. }\end{array}$ \\
\hline $\begin{array}{l}\text { United } \\
\text { States }\end{array}$ & $\begin{array}{l}\text { A complete list of public and private schools in East Baton Rouge Parish was assembled. Private } \\
\text { schools (collectively a stratum) were sampled separately. The public schools were sorted into quartiles } \\
\text { (strata) according to socio-economic status. Schools were randomly selected within each stratum until } \\
\text { a minimum of } 4 \text { schools were selected from each stratum, for a total minimum of } 20 \text { school. }\end{array}$ \\
\hline
\end{tabular}


Measurements were kept as consistent as possible across all study sites to ensure data were comparable; however, some questions were modified to better reflect the culture of the specific country (e.g., modifications of the diet-related questions to include local preferences). Some countries also added ancillary studies with additional measures. ISCOLE Canada added two ancillary studies: the concurrent use of Actical accelerometers, and an assessment of physical literacy. Data from the Actical accelerometers were not used in this analysis, but were collected as part of ISCOLE Canada to allow direct comparison with the Canadian Health Measures Survey. ${ }^{3,4}$ Data from the assessment of physical literacy were used in Manuscript 1. Physical literacy was measured using the CAPL battery of tests, which included the Knowledge and Understanding Questionnaire, as well as additional physical measurements. Details on CAPL measurements can be found below.

The results of ISCOLE provide a robust examination of the correlates of obesity in children, focusing on both sides of the energy balance equation (i.e., energy intake and energy expenditure)..$^{1}$ Details on measurement tools and protocols can be found below and include accelerometery, anthropometrics, and questionnaires (both child- and parent-report, Appendices D-F). Additional details on ISCOLE methodology can be found elsewhere. ${ }^{1}$

\subsubsection{Anthropometric measurements}

Anthropometrics (i.e., standing and sitting height, weight, waist circumference, upper arm circumference, percent body fat) were directly measured by trained and certified ISCOLE researchers during each school visit. Table 1 provides additional details 
of each measure.

Table 3.3. ISCOLE anthropometric measurement table.

\begin{tabular}{|l|l|l|l|}
\hline Variable & Measurement tool & Record in duplicate to & $\begin{array}{l}\text { Range } \\
\text { (repeat if) }\end{array}$ \\
\hline Standing height & Seca 213 Portable Stadiometer & Nearest $0.1 \mathrm{~cm}$ & $>0.5 \mathrm{~cm}$ \\
\hline Sitting height & $\begin{array}{l}\text { Seca 213 Portable Stadiometer, } \\
\text { Standardized bench }\end{array}$ & Nearest $0.1 \mathrm{~cm}$ & $>0.5 \mathrm{~cm}$ \\
\hline Waist circumference & Anthropometric tape & Nearest $0.1 \mathrm{~cm}$ & $>0.5 \mathrm{~cm}$ \\
\hline Arm circumference & Anthropometric tape & Nearest $0.1 \mathrm{~cm}$ & $>0.5 \mathrm{~cm}$ \\
\hline Body weight & Tanita Body Composition Analyzer (SC-240) & Nearest $0.1 \mathrm{~kg}$ & $>0.5 \mathrm{~kg}$ \\
\hline Body fat percentage & Tanita Body Composition Analyzer (SC-240) & Nearest $0.1 \%$ & $>2.0 \%$ \\
\hline
\end{tabular}

Height was measured using a Seca 213 Portable Stadiometer. Participants were asked to remove their shoes, and stand with their back towards the stadiometer, with feet together, their heels touching the base of the stadiometer, and their head aligned in the Frankfort plane (i.e., so that there is a horizontal line from the ear canal to the lower border of the orbit of the eye and it is parallel to the floor). Participants were asked to stand as tall as possible, with measurements being taken at the end of a gentle inspiration.

Sitting height was measured using a Seca 213 Portable Stadiometer and a standardized bench. Participants were asked to sit on the bench, with their back against the stadiometer, their legs hanging freely, their arms resting on their thighs, and their head aligned in the Frankfort plane. Participants were asked to sit as tall as possible, with measurements taken at the end of a gentle inspiration. Sitting height was used to calculate biological maturity of each child via an estimation of somatic maturation ${ }^{5}$ calculated by the percentage of predicted adult height, and maturity offset. ${ }^{6,7}$ As ISCOLE is a cross-sectional study, methods proposed by Mirwald et al. ${ }^{7}$ were used to predict maturity offset from age, sex, sitting height, stature and body mass. 
Although part of ISCOLE, and important to mention here, maturity offset was only used during preliminary data analysis as a covariate.

Weight and body fat percentage were measured using a portable Tanita Body Composition Analyzer (SC-240). Participants were asked to remove shoes, socks, and any heavy accessories (e.g., large sweaters) before stepping on the scale. The Tanita SC240 recorded participant's body weight, bioelectric impedance, and percent body fat. The Tanita SC-240 has shown acceptable accuracy for estimating body fat percentage when compared with dual-energy X-ray absorptiometry, supporting its use in fieldbased studies. ${ }^{8}$ The Tanita SC-240 was also non-intrusive and as such provided almost no missing data.

Waist circumference was measured at the mid-point between the top of the iliac crest and the bottom of the last floating rib using a non-elastic anthropometric tape. The iliac crest and floating ribs were palpated by an ISCOLE researcher, and marked with a washable marker. The mid-point between the marks was measured and marked and the anthropometric tape was placed around the participant's waist such that it was tight enough to stay in place, but not so tight as to indent the skin. Waist circumference was measured after a normal exhalation. Children were measured behind a temporary screen to provide privacy.

\subsubsection{Directly measured physical activity and sedentary behaviour}

Recent technological advances have allowed for widespread use of accelerometers to objectively measure time spent engaging in various activities. Accelerometers, worn attached to a small belt around the waist, are small monitoring 
devices used to measure movement accelerations of an individual. During data collection, the filtered movement intensities were stored as an activity count for a userspecified 'epoch' interval. Epoch-by-epoch activity counts were used to calculate each participant's volume and intensity of activity. From this information, the amount of time the participant spent engaging in varying intensities of activities, including PA (of all intensities), SED, and sleep was determined. ${ }^{9}$ Total sedentary time (SED) was defined as all epochs showing $\leq 25$ counts per 15 seconds, consistent with widely used cutoffs from Evenson et al. ${ }^{10}$ (i.e., sedentary: 0-25 counts/15 seconds, light: $26-573$ counts/15 seconds, moderate-to-vigorous: $\geq 574$ counts/15 seconds). For analysis, total SED was treated as a continuous variable. Manuscripts 1, 2, and 3 utilized accelerometer measured SED. Manuscript 1 also included accelerometer measured PA. Manuscripts 2 and 3 included sleep and PA to better understand correlates of SED and ST, although self-report values of both sleep and PA were included in the final models.

ISCOLE participants were asked to wear the accelerometers on an elastic belt, aligned above the right hip, 24-hours a day, removing only when doing water activities (e.g., bathing or swimming). Children were instructed to wear the accelerometers for seven consecutive days. At least four days of valid measurements (including at least one weekend day), with at least 10 hours of valid waking wear time per day, was needed for data to be included in the ISCOLE database. ${ }^{9}$ Non-wear time was defined by monitoring time with at least 60 consecutive minutes of zero counts. Sampling rate was set to $80 \mathrm{~Hz}$ with 1-second epoch lengths, aggregated to 15-second epochs for analysis. To increase compliance, study staff instructed children how to wear the accelerometer during the 
initial in-school assessment, and conducted an in-person compliance check 2-4 days after initialization. Further, participants were contacted twice via telephone (one weekday evening and one week end day) to ensure they were wearing the device, and to address any questions.

\subsubsection{Child- and parent-report questionnaires}

ISCOLE questionnaires included both child- and parent- report questionnaires. The Diet and Lifestyle Questionnaire (Appendix D) asked the child questions related to dietary intake, PA, SB, and sleep; the Demographic and Family History Questionnaire (Appendix E) and the Neighbourhood \& Home Environment Questionnaire (Appendix F) asked parents questions related to the home and neighbourhood environment. The child-report questionnaire was administered during an ISCOLE school-visit, at the same time that anthropometric measurements were taken and that accelerometers were given out. The parent-report questionnaires were sent home with the child at the same time as the parent-consent forms (i.e., prior to when the children were assessed). As the child finished their survey, and after collecting the parent-report surveys, ISCOLE researchers performed quality control checks on all parts of the questionnaires to ensure there were no missing data or invalid responses. In the case that there was missing data, parents were contacted via telephone for clarification.

Child-reported screen time was determined from the Diet and Lifestyle Questionnaire, with questions taken from the U.S. Youth Risk Behaviour Surveillance System. ${ }^{1,11}$ Children were asked how many hours they typically watched TV, and how many hours they played video games and/or used the computer per week day, and per 
weekend day for recreation. ${ }^{1}$ Responses were: $1=1$ did not watch TV, 2 = Less than 1 hour, $3=1$ hour, $4=2$ hours, $5=3$ hours, $6=4$ hours, $7=5$ or more hours. Response categories were collapsed to include $1=\leq 1$ hour of TV, $2=2$ hours, $3=3$ hours, $4=4$ hours, and $5=\geq 5$ hours. A weighted mean score of daily screen time was then calculated as follows: [(hours of TV on weekdays $x$ 5) + (hours of TV on weekend days $x$ $2)+($ hours of video games and computers on weekdays $\times 5)+($ hours of video games and computers on weekend days $\times 2$ )]/7. For analysis, this is presented as a screen time score, rather than total hours of ST since after 5 hours per day, we could not ascertain the participant's actual amount of ST.

All data collection methods used in ISCOLE were approved by the relevant research ethics boards at Pennington Biomedical Research Center, at each ISCOLE study site, and at the respective school boards. A complete list of institutional research ethics boards involved in the approval of this project can be found in Appendix G.

\subsubsection{Representativeness of ISCOLE}

By design, ISCOLE study sites were not intended to be representative of the site country. ${ }^{1}$ The primary sampling frame was schools, typically invited to participate in order to cover a range of socio-economic status to maximize variability within study sites. However, as ISCOLE represents the most up-to-date, largest, and most geographically diverse study of children around the world, we thought it was interesting, and important, to understand how representative ISCOLE site samples were when compared to available national samples. Therefore, the second part of the background work that was completed to help inform this dissertation looked at the 
representativeness of ISCOLE participant characteristics in each country. The full results of this work can be found in Appendix A and have been submitted for publication in a special issue of the International Journal of Obesity. In brief, the purpose of the background paper was to examine if data collected at ISCOLE study sites was representative of the site country as a whole. In a broader sense, it is hoped that this work can be used to better understand the limitations, potential biases and generalizability of results of ISCOLE, and to understand current gaps in knowledge in respective countries. More specific to the work presented within this dissertation, it was hoped that by understanding if ISCOLE participants were representative of children around the world, I could provide more significant and applicable public health messages related to reducing SED and ST.

Of the 12 ISCOLE countries, five provided nationally representative data on objectively measured PA, nine provided data on self-reported SB, and eight provided data on weight status to allow comparison with the ISCOLE sample. The five ISCOLE countries that were part of the Health Behaviours in School-aged Children also provided data on self-reported PA. Due to the limited availability of country-level data, it is suggested that ISCOLE data be used with appropriate caution when planning countrywide population health interventions. However, for some countries, ISCOLE currently provides the most up-to-date and most robust data on obesity and PA in children. This work has identified the paucity of comparable country data around the world and highlighted the importance of large, multi-national studies like ISCOLE.

\subsubsection{Canadian Assessment of Physical Literacy (CAPL)}


The CAPL is a battery of tests designed to assess physical literacy, or the capacity to achieve a healthy, active lifestyle, in children and youth. ${ }^{12}$ The CAPL assesses physical literacy with four key domains: fitness (endurance, strength, flexibility, body composition), motor skill (gross motor skills), habitual activity (pedometer measured PA, self-reported SB), and cognition (attitudes, knowledge, and motivations). ${ }^{13}$ As part of the CAPL, children are asked to complete a Knowledge and Understanding questionnaire, with questions relating to their awareness of current Canadian PA and SB guidelines.

CAPL data used for this project were collected across elementary schools in 2011-2013 in Ottawa, Canada. Initial CAPL data collection occurred between 2011-2012 and included students in grades 4-6 from elementary school across the greater Ottawa area $(n=680,47 \%$ boys, mean age $=10.1$ years). Additional CAPL data collection occurred as an ancillary study as part of ISCOLE Canada. Children from the English Catholic and English Public school boards who consented to participate in ISCOLE were asked if they would like to participate in CAPL with a recruitment goal of 200 children (actual $n=229,37 \%$ male, mean age $=10.0$ years). Accelerometer data on PA, SED, and sleep were taken for these participants from the ISCOLE database.

Data used for the current analyses were taken from the Knowledge and Understanding questionnaire (Appendix $\mathrm{H}$ and https://www.capl-ecsfp.ca/caplmanual/previously named the Cognitive Domain Questionnaire), which was administered during class time by a trained researcher. Children were asked the main messages of current PA and SB guidelines. It is important to note that the questionnaire 
focused on the main message of the PA guideline (i.e., participating in 60 minutes of MVPA per day), and about the ST within the SB guidelines (i.e., watching $\leq 2$ hours of recreational ST per day). Children were not asked about all caveats of the guidelines.

Children were asked what they would do to improve a sports skill, what they would do if they wanted to improve their fitness and what activity they would prefer to do after school (e.g., watching TV or walking their dog) to reflect on their current activity level, and to compare their skills related to physical activity to their peers. Additional details on questions and data reduction procedures can be found in Manuscript 1.

\subsection{Analytical plan}

SAS 9.4 (SAS Institute Incorporated, North Carolina, USA) was used for all statistical analyses. Descriptive information (means, standard deviations, frequencies as appropriate) was calculated for demographic and anthropometric characteristics of all participants and their parents where applicable. Unpaired t-tests and chi-square tests were used to test differences between boys and girls. For manuscript 1, in the ISCOLECAPL subsample, analysis of covariance (ANCOVA) was used to determine the relationship between habitual activity (MVPA, SED, and ST), and knowledge of guidelines (PA and ST), and self-perceptions of PA. All manuscripts employed multi-level general linear models (PROC MIXED) to account for the hierarchical nature of the data. All analysis will be done for the total sample, and then split by boys and girls to account for known differences in levels of SED and ST, as well as differences in potential correlates. ${ }^{14}$ Presenting the analysis for boys and girls separately may also provide insights for future research. 


\subsubsection{Multilevel modeling - background}

In the last twenty years, multi-level modeling has become a popular analysis to account for hierarchically organized data. ${ }^{15-17}$ For example, it is not uncommon to collect data on students, nested within schools, and schools nested within school boards. To ignore the hierarchical nature of these data can lead to erroneous conclusions such as incorrect interpretation of the estimated variances, inflation of the likelihood of making Type 1 errors, and/or errors in interpretations of statistical significance. ${ }^{18}$ In the simplest terms, multilevel models can be thought of as simple regression models occurring at different levels. Multilevel models can then differ by the number of levels that are included, the type of study design (e.g., cross- sectional or longitudinal), the type of outcome variable (e.g., continuous, dichotomized), and the number of exposure variables. ${ }^{18}$ Multilevel models can also be used to help understand how much variance is occurring at each level of the model - this is explained in detail below.

\subsubsection{Use in population health research}

Multilevel modeling helps researchers understand how individuals are influenced by their social context. Further, multilevel modeling can be used to understand how groups are related to other groups, or how individuals are related to each other within groups. This allows researchers to examine the relevant distributions of health outcomes. The distribution of variance in a health outcome should be calculated before the overall models are even built to understand how much variance in the dependent variable can be attributed to each level that has been included in the 
model. To do this, interclass correlation coefficients (ICC) are calculated. In my work, ICCS were calculated to understand how much variance in level of SED and ST was due to individual differences. This can also be used to determine the potential impact at school or country level interventions. Finally, calculating the ICCs of dependent variables can also show if a multilevel analysis is needed. If the ICC is zero, then no variation exists at higher levels (e.g., school or country) and all the variation exists at the individual level, in which case multilevel analysis is not required.

Table 3.4 provides a partial SAS output to show how to calculate interclass coefficients were calculated in Manuscript 3 for SED (with both school and site as random effects). Similar calculations were completed for Manuscript 2, and to determine the variance in ST.

Table 3.4. SAS output to calculate interclass correlation coefficients.

\begin{tabular}{|l|l|r|}
\hline \multicolumn{3}{|c|}{ Covariance Parameter Estimates } \\
\hline Cov Parm & Subject & Estimate \\
\hline Intercept & school_ID(SITE_ID) & 433.82 \\
\hline Intercept & SITE_ID & 631.48 \\
\hline Residual & & 3781.01 \\
\hline
\end{tabular}

$$
\begin{aligned}
\mathrm{ICC}_{\text {school }} & =\frac{\sigma^{2} \text { school }}{\sigma_{\text {estimate }}+\sigma^{2} \text { school }+\sigma^{2}{ }_{\text {site }}} \\
& =\frac{433.82}{4,846.31} \\
& =0.0895(\approx 9 \%) \\
\mathrm{ICC}_{\text {site }} & =\frac{\sigma^{2} \text { site }}{\sigma_{\text {estimate }}+\sigma^{2}{ }_{\text {school }}+\sigma^{2} \text { site }} \\
& =\frac{631.48}{4,846.31} \\
& =0.1303(\approx 13 \%)
\end{aligned}
$$


This indicates that approximately $78 \%$ of the variability in total SED can be attributed to individual level differences, $9 \%$ can be attributed to school level differences, and $13 \%$ attributed to country level differences. This information can also help when interpreting and making recommendations for public health interventions.

\subsubsection{Model building process}

A quick scan of recent research shows that there are many different approaches to the model building process. However, the ultimate goal is always to estimate the model(s) that best fit the data. Bell et al. ${ }^{18}$ provide a comprehensive guide on model building using SAS (PROC MIXED). For this work, the model building process was partly informed by the socio-ecological model, as proposed by Owen et al. ${ }^{19}$ This is in line with the assumption that children's habitual behaviours are a result of multiple competing and interacting influences. Below is an example of the models that were used in this work.

Table 3.5. Model building process ${ }^{18}$

Model 1

- No predictors, just the random effect for the intercept

- The output from this is used to calculate the interclass coefficients to understand how much variance is occurring at each level
Model 2

- Model + level 1 random effect.

- This is used in Manuscript 1, keeping school as a random effect.

- Adding in different potential correlates as they pertain to different socio-ecological domains.

- Can be used with, or without covariates.
Model 3

- Model + level 1 and level 2 random effects.

- This is used in Manuscript 3, keeping school and ISCOLE site as a random effect.

- Adding in different potential correlates as they pertain to different socio-ecological domains.

- Can be used with, or without covariates.

First, the model is run with no predictors to calculate ICCs. Then, we added

various correlates, along with potential correlates we wanted to test. For manuscript 2, 17 potential correlates were tested within the model; for manuscript 3 , this list was 
expanded to include some additional neighborhood variables and included 21 potential correlates. The majority of information for potential correlates was obtained via childand parent-report questionnaire. Details on all potential correlates, how the information was collected, and how they were treated in analysis can be found in Manuscripts 2 and 3. For Manuscripts 2 and 3, accelerometer measured SED, and selfreported ST were included as dependent variables. To satisfy the assumption of normality required in multilevel modeling, ST was log transformed for analysis. ${ }^{18}$ Analysis for SED and ST were completed separately. To test associations between potential correlates and total SED or ST, potential correlates were first included in univariate models (including relevant covariates); variables that were at least marginally significant $(p<0.10)$ were subsequently included in domain-specific models similar to those outlined in the socio- ecological model proposed by Owen et al. for SED ${ }^{19}$ (i.e., biological characteristics, behavioural characteristics, parental characteristics, and home and neighborhood environment). Variables that showed at least marginally significant associations $(p<0.10)$ with SED or ST in the domain-specific models were included in the final model. Variables that remained significant $(p<0.05)$ in the final model were considered correlates of SED and/or ST. In Manuscript 3, to show differences in levels of SED and ST between boys and girls in each site, we ran sex by site interactions and computed the least square means (LSMEANS) of fixed effects. We also ran the final models separately in each ISCOLE site to determine which correlates were the most common. 
Multilevel analysis summarizes the distribution in terms of two parts - a fixed part that is unchanging across groups, and a random part that is allowed to vary from group to group. For Manuscript 2, school was treated as a random effect; Manuscript 3 had an additional level and both school and ISCOLE site were treated as random effects. This allowed us to calculate associations across the entire ISCOLE dataset. If we had wanted to look at differences between countries, we would have kept ISCOLE site as a fixed effect. However, since we were examining such a large number of potential correlates, this was not feasible. Appendix I provides a sample SAS code that was run with additional analysis notes.

Within the model statement, it is also important to specify which estimation should be used to handle degrees of freedom (of which SAS provides many options). A more comprehensive explanation can be found elsewhere but it should be mentioned that the two most commonly used estimates are Satterthwaite and the Kenward-Roger because they are well suited to be used in models with unbalanced designs and are able to handle complex covariance structures. ${ }^{20}$ The Kenward-Roger approximation (DDFM=KR) was used here as it also adjusts for small sample size bias. ${ }^{18}$

\subsubsection{Strengths and limitations of multilevel modeling}

The biggest strength of multilevel modeling is that it allows for the simultaneous examination of the effects of group level and individual level predictors on your dependent variable. This helps researchers to avoid the ecological fallacy, which is inherent in most other regression models. The ecological fallacy occurs when inferences from the nature of individuals (e.g., reasons for increased SED) are deduced from 
inferences from the group to which the individual belongs. ${ }^{15}$ Multilevel models also tend to outperform traditional regression in predictive accuracy. Further, traditional analysis models can produce excessive Type 1 errors and biased parameter estimates..$^{21,22}$ However, multilevel modeling does come with some limitations. First of all, statistical techniques used in multilevel modeling are relatively advanced and therefore difficult for many researchers. The complexity of statistical knowledge needed to run multilevel modelling also means that there is opportunity to misinterpret findings. Multilevel modeling also assumes normality in dependent variables; meaning researchers may have to transform (and back transform) their data for analysis and interpretation. Finally, calculation of effect size is not as straightforward as with other regression models and currently no consensus exists to the most appropriate approach to use..$^{22}$ Multilevel modeling procedures, as they relate to each manuscript, are explained further in Chapter 4. 


\section{References}

1. Katzmarzyk, P. T. et al. The International Study of Childhood Obesity, Lifestyle and the Environment (ISCOLE): design and methods. BMC Public Health 13, 900 (2013).

2. Shields, M. \& Tremblay, M. S. Canadian childhood obesity estimates based on WHO, IOTF and CDC cut-points. Int. J. Pediatr. Obes. 5, 265-273 (2010).

3. Tremblay, M., Wolfson, M. \& Connor Gorber, S. Canadian Health Measures Survey: rationale, background and overview. Health Rep. 18 Suppl, 7-20 (2006).

4. Giroux, S. Canadian Health Measures Survey: sampling strategy overview. Health Rep. 18 Suppl, 31-36 (2006).

5. Malina, R., Bouchard, C. \& Bar-Or, O. Growth, Maturation, and Physical Activity. (Human Kinetics, 2004).

6. Khamis, H. J. \& Roche, A. F. Predicting Adult Stature Without Using Skeletal Age: The Khamis-Roche Method. Pediatrics 94, 504-507 (1994).

7. Mirwald, R. L., Baxter-Jones, A. D. G., Bailey, D. A. \& Beunen, G. P. An assessment of maturity from anthropometric measurements. Med. Sci. Sports Exerc. 34, 689-694 (2002).

8. Barreira, T. V., Staiano, A. E. \& Katzmarzyk, P. T. Validity assessment of a portable bioimpedance scale to estimate body fat percentage in White and African-American children and adolescents. Pediatr. Obes. 8, e29-e32 (2013).

9. Tudor-Locke, C. et al. A model for presenting accelerometer paradata in large studies: ISCOLE. Int. J. Behav. Nutr. Phys. Act. 12, 52 (2015). 
10. Evenson, K. R., Catellier, D. J., Gill, K., Ondrak, K. S. \& McMurray, R. G. Calibration of two objective measures of physical activity for children. J. Sports Sci. 26, 1557-1565 (2008).

11. Centers for Disease Control and Prevention. Youth Risk Behavior Surveillance System (YRBSS). U.S. Centers for Disease Control and Prevention (2012). at <http://www.cdc.gov/healthyyouth/data/yrbs/index.htm>

12. Longmuir, P. E., Boiaraskia, E., Zhu, W., Lloyd, M. \& Tremblay, M. S. Canadian Assessment of Physical Literacy: A Model and Methods for Assessing the Physical Literacy of Children 8 to 12 Years of Age. Unpublished (Unpublished).

13. Lloyd, M., Colley, R. C. \& Tremblay, M. S. Advancing the debate on 'fitness testing' for children: perhaps we're riding the wrong animal. Pediatr. Exerc. Sci. 22, 176182 (2010).

14. Temmel, C. S. D. \& Rhodes, R. Correlates of Sedentary Behaviour in Children and Adolescents Aged 7-18: A Systematic Review. Health Fit. J. Can. 6, 119-199 (2013).

15. Diez-Roux, A. V. Multilevel Analysis in Public Health Research. Annu. Rev. Public Health 21, 171-192 (2000).

16. Pickett, K. E. \& Pearl, M. Multilevel analyses of neighbourhood socioeconomic context and health outcomes: a critical review. J. Epidemiol. Community Health 55, 111122 (2001).

17. Leyland, A. H. \& Groenewegen, P. P. Multilevel modelling and public health policy. Scand. J. Public Health 31, 267-274 (2003). 
18. Bell, B. B., Ene, M., Smiley, W. \& Schoeneberger, J. A. A multilevel model primer using SAS PROC MIXED. (University of South Carolina, 2013).

19. Owen, N. et al. Adults' Sedentary Behavior: Determinants and Interventions. Am. J. Prev. Med. 41, 189-196 (2011).

20. Kenward, M. G. \& Roger, J. H. Small Sample Inference for Fixed Effects from Restricted Maximum Likelihood. Biometrics 53, 983 (1997).

21. Gelman, A. Multilevel (Hierarchical) Modeling: What It Can and Cannot Do. Techometrics 48, 432-435 (2006).

22. Peugh, J. L. A practical guide to multilevel modeling. J. Sch. Psychol. 48, 85-112 (2010). 


\section{Chapter 4}

\section{Research Results}

4.1 Canadian physical activity and screen time guidelines: are children aware?

This manuscript has been written and formatted to adhere to the specifications for submission to the Journal of School Health (currently under review). 
Canadian physical activity and screen time guidelines: are children aware?

Allana G LeBlanc ${ }^{1,2^{*}}$, Charles Boyer ${ }^{1}$, Michael M Borghese ${ }^{1}$, Jean-Philippe Chaput ${ }^{1,3}$, Genevieve Leduc ${ }^{1}$, Mark S Tremblay ${ }^{1,3}$, Patricia E Longmuir ${ }^{1,3}$

${ }^{1}$ Healthy Active Living and Obesity Research Group, Children's Hospital of Eastern Ontario Research Institute, Ottawa, Ontario, Canada. ${ }^{2}$ Faculty of Graduate and Postdoctoral Studies, University of Ottawa, Ottawa, Ontario, Canada.

${ }^{3}$ Department of Pediatrics, University of Ottawa, Ottawa, Ontario, Canada.

\author{
*Correspondance \\ Allana G LeBlanc, PhD (c), MSc, CSEP-CEP, ACSM-CES \\ Healthy Active Living and Obesity Research Group \\ Children's Hospital of Eastern Ontario Research Institute \\ 401 Smyth Road, Ottawa, Ontario, Canada \\ K1H 8L1 \\ Phone: +1 6137377600 ext. 4191 \\ Fax: +16137384800 \\ E-mail: alleblanc@cheo.on.ca
}


Title: Canadian physical activity and screen time guidelines: do children know?

Running head: Awareness of guidelines in Canada

Type of submission: original research

Manuscript word count: 3,756

Abstract word count: 200

Date of submission: August 2015

Key words: physical activity guidelines, sedentary behaviour guidelines, awareness, children

BACKGROUND: A recent review reported that few parents, or caregivers are aware of current physical activity (PA), or screen time (ST) guidelines. No study has examined children's awareness. This study aimed to determine the proportion of Canadian children who could correctly identify PA and ST guidelines.

METHODS: Data were obtained through the Canadian Assessment of Physical Literacy $(C A P L)(n=787,46.6 \%$ boys) and a CAPL sub-sample of the International Study of Childhood Obesity, Lifestyle and the Environment (ISCOLE) ( $n=223,38.1 \%$ boys). PA and sedentary behaviour were measured by accelerometry; ST was measured via self-report. RESULTS: Most children (71.6\%) correctly identified PA guidelines; $20.0 \%$ correctly identified ST guidelines. The majority of children knew they should try exercising and being more active to improve a sports skill (63.7\%) or improve their fitness (88.2\%). In adjusted models, children's ability to correctly identify guidelines did not differ by activity level. Adjusted models showed significant associations between PA, ST, and selfperceptions of PA. No associations were found with sedentary time. 
CONCLUSION: Regardless of activity level, children correctly identified current PA guidelines; fewer children could identify ST guidelines. Given the low proportion of children meeting current guidelines, it may be beneficial to highlight them in the school curriculum. 
With the aim of helping parents, caregivers, and health care providers to improve activity habits of children, the Canadian Society for Exercise Physiology (CSEP) released evidence-informed Canadian physical activity (PA) and sedentary behaviour (guidelines for children and youth. ${ }^{1-3}$ The guidelines are based on the best available evidence, and developed in partnership with researchers, government representatives, and stakeholders from across Canada. ${ }^{4,5}$

The PA guidelines recommend that children and youth participate in at least 60 minutes of moderate- to vigorous-intensity physical activity (MVPA) daily, including incorporating vigorous-intensity activities, and activities that strengthen muscle and bone at least three days per week. ${ }^{1}$ Meeting the guidelines is associated with better cardio-metabolic health, lower prevalence of overweight/obesity, and improved bone health and mental health. ${ }^{4}$ Sedentary behaviour guidelines recommend that children and youth should minimize the time they spend being sedentary each day. Specifically, the sedentary guidelines recommend limiting recreational screen time (ST) to no more than 2 hours per day, limiting sedentary (i.e., motorized) transport, extended time spent sitting, and time spent indoors throughout the day. ${ }^{2}$

Since the release of the PA and sedentary behaviour guidelines, many studies in Canada, and elsewhere, have examined the health benefits of adhering to the guidelines; however, little work has been done to examine children's own knowledge or understanding of the guidelines. A recent review showed the paucity of research in this area, and highlighted low awareness of PA and sedentary behaviour guidelines from parents, the general public, and health care providers. ${ }^{6}$ Carson et al., ${ }^{7}$ examined 
paediatrician awareness, agreement, and use of the PA and sedentary behaviour guidelines and reported that less than $10 \%$ of those sampled were familiar with the PA and sedentary behaviour guidelines for the early years, children, and youth. Results from a ParticipACTION report also showed low awareness of the guidelines among the general population and mothers. ${ }^{6}$ Eight percent of the general public and $12 \%$ of moms responded that they had heard of the PA guidelines; $3 \%$ of the general public and $3 \%$ of moms had heard of the sedentary behaviour guidelines. The review was not able to identify any studies that asked children directly about their knowledge, or understanding of the guidelines. ${ }^{8}$

The primary aim of this study was to determine the proportion of Canadian children who could correctly identify current PA and sedentary behaviour guidelines (specifically related to ST guidelines) in prompted questioning. Children were also asked questions about their self-perceptions of PA, and how they could improve their sports skills and fitness level. In a subsample of participants, we examined if guideline knowledge, or self-perceptions of PA were associated with accelerometer measured PA, accelerometer measured total sedentary time (SED), or self-reported ST.

\section{METHODS}

\section{Participants}

\section{Canadian Assessment of Physical Literacy}

The Canadian Assessment of Physical Literacy (CAPL) is a battery of tests designed to assess physical literacy defined here as the capacity to achieve a healthy, active lifestyle, in children and youth. ${ }^{9,10}$ Data used here were collected in 2011-2012 
through elementary schools (grades 1-8, ages 6-14 years) across Ottawa, Ontario.

Selected English Catholic schools in Ottawa and English public schools in Eastern Ontario were invited to participate in the CAPL assessments and all students in the classes who volunteered to participate received a study information package to take home. The information package explained the study procedures and included the parent consent form. Children who completed the CAPL assessment were included in the current analysis ( $n=787,47.2 \%$ boys). This analysis included CAPL assessments for motivation and confidence (measured via self-report questionnaire), and knowledge, awareness and understanding of PA (measured via self-report questionnaire).

\section{The International Study of Childhood Obesity, Lifestyle and the Environment}

The International Study of Childhood Obesity, Lifestyle and the Environment (ISCOLE) is a multi-national, cross-sectional study conducted in 12 countries around the world. Details on ISCOLE methodology have been published elsewhere. ${ }^{11}$ Data for ISCOLE were collected from Grade 5 students (aged 9-11 years) from four school boards: English Public, French Public, English Catholic, and French Catholic across the Ottawa, Ontario region. As part of ISCOLE-Canada, the CAPL was administered as an ancillary study such that schools from the English Public, and the English Catholic schools that participated in ISCOLE were invited to participate in CAPL until a sample size of 200 children was reached. Data collection for ISCOLE-CAPL occurred in spring 2013 with about half of those who consented to participate in ISCOLE providing complete CAPL data ( $n=223,38.1 \%$ boys). Additional information on daily activity (i.e., accelerometer measured information on PA and SED, self-reported ST), parental education, and 
maturity offset was also extracted for these children from the main ISCOLE-Canada database.

Ethics approval for CAPL, ISCOLE, and ISCOLE-CAPL was provided by the research ethics board at the Children's Hospital of Eastern Ontario, the University of Ottawa, and the participating school boards. Written informed parental consent and child assent were obtained from all participants.

\section{Instrumentation}

\section{Knowledge of the guidelines and perceptions of physical activity}

The Knowledge and Understanding questionnaire (https://www.caplecsfp.ca/capl-manual//) was administered during class time by a trained researcher; children were instructed to read and answer the questions independently, but clarification was provided if requested. The researcher emphasized that the questions asked about the children's own opinions, that there were no right or wrong answers, and if uncertain, children should answer to the best of their knowledge (multiple choice answers were provided). Questions regarding knowledge of PA and ST guidelines were re-coded as binary variables to reflect correct (and incorrect) responses. It is important to note that the questionnaire only asked about the main message of the PA guidelines (i.e., accumulating 60 minutes of MVPA daily), and about the screen time within the SB guidelines (i.e., limiting recreational screen time to no more than 2 hours per day). In other words, children were not asked about all aspects of the guidelines.

Children were asked about what they would do to improve a sports skill, and what they would do if they wanted to improve their fitness. For both questions, there 
were four possible responses: "read a book", "wait until you get older", "try exercising or being more active", or "take a lesson". Children were also asked what they would prefer to do after school. Answers to this question were dichotomized as "sedentary" or "active" (sedentary: play video/computer games, read, homework, chat with friends online, watch TV; active: play with friends at the playground, go to my sports team's practice, walk my dog). Questions relating to self-perceptions of PA were asked on a scale of 1-10 (1 being the lowest, 10 being the highest). Participant responses were analyzed as a continuous variable but participant response rate is presented in tertiles, to reflect the wording of the questions. Importance of increasing current activity is presented as: "I'm already active enough" (score: 1-3), "I need to be a little more active" (score: 4-7), and "I need to be a lot more active" (score: 8-10). Comparing their own skill level to that of their peers is presented as: "others are better" (score: 1-3), "same" (score: 4-7), and “I'm a lot better" (score: 8-10).

\section{Anthropometrics}

Anthropometrics were measured by trained researchers according to standard procedures. ${ }^{11}$ In both CAPL, and ISCOLE-CAPL, height was measured to the nearest 0.1 $\mathrm{cm}$ using a SECA portable stadiometer (Medical Scales and Measuring Systems, England). In CAPL, weight was measured to the nearest $0.1 \mathrm{~kg}$ with a digital scale (A\&D Medical Engineering Inc, USA). In ISCOLE-CAPL, weight (to the nearest $0.1 \mathrm{~kg}$ ) was measured using a portable Tanita SC-240 Body Composition Analyzer (Arlington Heights, IL, USA). Body mass index (BMI) was calculated $\left(\mathrm{kg} / \mathrm{m}^{2}\right)$ and age- and sex-specific BMI zscores were determined using the CDC growth charts. ${ }^{12}$ 


\section{Habitual activity}

Participants from the ISCOLE-CAPL subsample were asked to wear the ActiGraph GT3X+ triaxial accelerometer (ActiGraph LLC, Pensacola, FL, USA). Participants wore the accelerometer, attached to an elastic belt around the waist (at the right mid-axillary line), for 7 consecutive days, 24 hours per day, removing only for aquatic activities (e.g., bathing, swimming). ${ }^{13,14}$ To increase compliance, study staff instructed children on how to wear the accelerometer during the initial in-school assessment, conducted an inperson compliance check and contacted participants twice via telephone to ensure they were wearing the device, and to address any questions. To be included in the ISCOLE dataset, participants were required to provide at least four days of valid measurements (including at least one weekend day), with at least 10 hours of waking wear time per day. ${ }^{15,16}$ Data were collected at a sampling rate of $80 \mathrm{~Hz}$, downloaded in 1-second epochs, and aggregated to 15 -second epochs. ${ }^{17}$ moderate- to vigorous-intensity PA (MVPA) and SED were defined consistent with widely used cut-offs from Evenson et al., ${ }^{17}$ (i.e., sedentary: 0-25 counts/15 seconds, light: $26-573$ counts/15 seconds, moderate-to-vigorous: $\geq 574$ counts/15 seconds).

\section{Self-reported screen time}

Child-report screen time was captured as part of the ISCOLE Diet and Lifestyle Questionnaire and included here to examine its relationship with knowledge of screen time guidelines ${ }^{11}$. Children were asked how many hours they typically watched television (TV), and how many hours they played video games and/or used the computer per week day, and per weekend day. Responses were: $0=1$ did not watch TV, 
$1=$ less than, or one hour of TV, $2=2$ hours, $3=3$ hours, $4=4$ hours, $5=5$ or more hours of TV. A weighted mean score of hours of screen time per week was then calculated as follows: [(hours of TV on weekdays $\times 5)+($ hours of TV on weekend days $\times 2)+($ hours of video games and computers on weekdays $\times 5)+($ hours of video games and computers on weekend days $\times 2$ )]/7. For analysis, this is presented as a screen time score, rather than total hours of screen time since after 5 hours per day, we could not ascertain the participant's actual amount of screen time. After testing for normality, ST was logtransformed for analysis and analyzed as a continuous variable. ST was also presented as those who did not meet ST guidelines of $\leq 2$ hours/day. ${ }^{2}$

\section{Additional covariates}

Sex, waist circumference, maturity offset, parental education (mother and father) were included as covariates in models when determining the relationship between activity status, knowledge of the guidelines, and perceptions of PA. Biological maturity was estimated using the maturity offset method, which estimates an individual's age from peak height velocity (from height, sitting height, body mass, and chronological age). ${ }^{18}$ Maturity offset was included as a covariate instead of age since the age range for the ISCOLE-CAPL subsample was so narrow, and maturity has been shown to be significantly associated with habitual activity. ${ }^{19}$ Highest parental-report education was used to estimate socio-economic status.

\section{Data analysis}

Statistical analyses were completed using SAS 9.4 (SAS Institute Inc., North Carolina, U.S.A). Descriptive information (mean, standard deviation) was calculated for 
demographic and anthropometric characteristics of all participants. Independent samples t-tests and chi-squared tests were used to determine differences between CAPL and ISCOLE-CAPL, and between boys and girls. In the ISCOLE-CAPL subsample, additional analysis was performed related to guideline awareness. Multilevel general linear models (PROC MIXED), including school as a random effect, were used to determine the relationship between guideline awareness and measures of directly measured activity status (i.e., MVPA and SED), ST, and self-perceptions of PA. ${ }^{20}$ The Kenward-Roger approximation (DDFM=KR) was used to calculate degrees of freedom. ${ }^{21}$ Multicollinearity was tested using tolerance and variance inflation factors, and results indicated no issues with multicollinearity. ${ }^{22}$ Interclass correlation coefficients were calculated to determine the amount of variation in PA, SED, and ST that was attributable to the individual, and to the school level.

\section{RESULTS}

Participant characteristics are presented in Table 1. Children from ISCOLE-CAPL $(n=223)$ had smaller waist circumferences [mean difference $=-6.7 \mathrm{~cm}(95 \% \mathrm{Cl}:-6.15,-$ 3.23), $\mathrm{p}<0.0001)$ ] and lower BMI $z$-score [mean difference $=-0.26(95 \% \mathrm{Cl}:-.042,-0.11)$, $p=0.0008$ ] than children from the CAPL $(n=787)$ group. Within the ISCOLE-CAPL sample, boys had a higher waist circumference $(p=0.021)$ and BMI $z$-score $(p=0.021)$ than girls. There were no differences between boys and girls within the CAPL sample. In the ISCOLE-CAPL subsample, the largest fraction of the total variance in MVPA, sedentary behaviour, and screen time was explained by individual-level factors (89\%, 89\%, and $88 \%$ respectively), with the remainder explained by school level differences. 


\section{Knowledge of physical activity and screen time guidelines}

Data on the children's knowledge of PA and ST guidelines, including participants from CAPL and ISCOLE-CAPL, are presented in Table 2. In prompted questioning, the large majority of boys (72.3\%) and girls (70.8\%) were able to correctly identify that they should accumulate 60 minutes of MVPA daily. Fewer children were able to correctly identify ST guidelines; boys were more likely to correctly identify ST guidelines than girls (22.8\% of boys, and $17.7 \%$ of girls, $\mathrm{p}=0.0475)$. However, a large proportion of children (65.2\% of boys and $72.7 \%$ of girls) identified that the guidelines recommend 30 minutes, or 1 hour or less of screen time per day. While this is incorrect, it is in line with the overarching recommendation of the sedentary behaviour guidelines that recommends that children minimize the time they spend sedentary each day.

\section{Perceptions of physical activity}

Children's responses to the Knowledge and Understanding of PA questionnaire, including participants from CAPL and ISCOLE-CAPL, are presented in Table 2. When asked what the best thing to do to improve a sport skill, most children (63.7\%) said that they should try exercising and being more active. Eight-eight percent of children also stated that they should try exercising and be more active if they wanted to get in better shape. The majority of children $(64.8 \%$ of boys and $71.7 \%$ of girls, $p=0.0237)$ stated that if given the choice, after school they would pick an active pursuit like playing with friends, or walking their dog. When comparing themselves to their peers, boys were more likely to report they were "a lot better" at sports or skills ( $49.3 \%$ of boys versus $28.6 \%$ of girls, $p<0.0001)$. 


\section{Measured physical activity}

Of the 223 children who participated in ISCOLE-CAPL, 202 had complete accelerometer data. On average, they accumulated 58 minutes of MVPA and 8.5 hours of SED per day. Boys engaged in significantly more MVPA than girls $(p<0.0001)($ Table 1$)$. In adjusted models, neither level of MVPA, nor SED were associated with being able to correctly identify PA guidelines (Figure 1, Panel A), or ST guidelines (Figure 1, Panel B). Similarly, in adjusted models, ST did not differ between those who could correctly identify PA or ST guidelines (data not shown as screen time score was log transformed for analysis). The relationship between MVPA and SED remained non-significant when including those who reported screen time guidelines as 30 minutes, or 60 minutes as having correctly identified the guidelines; however, with this new categorization, there was a negative relationship between ST and awareness of ST guidelines $(\beta=-0.25$, standard error $=0.12, \mathrm{p}=0.0277)$.

Associations between activity level and perceptions of PA are presented in Table 3. After adjusting for covariates, children with lower levels of MVPA were less likely to report that they were a more active than their peers. Similarly, children who reported higher levels of ST were less likely to report that they were more active, or more skilled than their peers. Children reporting higher levels of ST were also more likely to correctly identify strategies to increase their fitness than children who reported lower levels of ST. No associations were found with SED.

\section{DISCUSSION}


This work shows that the large majority (71.6\%) of children could correctly identify PA guidelines; fewer (20.0\%) were able to correctly identify ST guidelines of 2 hours per day, but the majority (89.4\%) correctly identified the guidelines as $\leq 2$ hours per day. We also found that most children could correctly identify strategies to increase their sports skills and fitness levels (i.e., try exercising or being a lot more active), and if given the choice would pick an active after-school pursuit. Furthermore, we determined that activity level was not associated with knowledge of the guidelines, but was associated with some self-perceptions of PA.

Overall, children seemed to have fairly accurate self-perceptions of their activity status. This was especially true when looking at MVPA and ST, where children could correctly self-identify as being more, or less active than their peers. It was interesting that children who reported higher levels of ST were more likely to correctly identify strategies to improve their fitness, perhaps because they've had more discussions about fitness than children who are already considered active. It was also interesting that we found no significant associations with SED. This is consistent with previous work suggesting that at least in children, ST, and not SED may be the more important behaviour. $^{23}$

Compared to previous studies examining parental knowledge of Canadian PA and sedentary behaviour guidelines, ${ }^{24,25}$ we found that the proportion of children able to correctly identify the guidelines was much higher. We could not find other studies examining children's awareness of PA guidelines (in Canada, or elsewhere), so it is possible that low levels of awareness are limited to adults. This may be in part due to 
the fact that children are taught about PA during school, whereas adults may not have been recently exposed to guideline-specific information. It is also possible that children simply guessed the correct answer. This is a limitation associated with prompted questioning and the questionnaires used for this study. However, previous results from prompted questioning in adults show awareness at close to $30 \%$, which is still much lower than what was shown in the current analysis. ${ }^{6}$

We speculate that there are several potential reasons why most children were not able to correctly identify ST guidelines. The first is that the overarching message associated with the sedentary behaviour guidelines is to reduce sedentary behaviour, limiting ST to no more than 2 hours per day is a bullet point of the guidelines, and therefore it is possible that it is lost in the messaging. Work from ParticipACTION showed that the general population, as well as moms of children aged 5-11 years, were most likely to identify the statement "lower levels of screen time are associated with additional health benefits" as the main component of the sedentary behaviour guidelines, and not "limit screen time to less than two hours per day". ${ }^{2}$

Future work should examine which components of the guidelines children are able to identify, and what can be done to improve this knowledge. Another possible reason why children are unaware of ST guidelines is that they are entirely unaware of the concept of sedentary behaviour, or sedentary behaviour guidelines. ParticipACTION showed that $70 \%$ of the general population, and $66 \%$ of moms had never heard of the sedentary behaviour guidelines and did not know the main components of the guidelines. ${ }^{6}$ Low levels of awareness of the SB guidelines may be in part due to the 
terminology used; children may be familiar with the concept of screen time but they may not understand the concept of SB as a distinct health risk factor. The concept of sedentary behaviour is relatively new, and only recently have researchers come to consensus on its definition. ${ }^{26}$ Therefore it is possible that sedentary behaviour guidelines are undervalued by health promoters (e.g., teachers, parents, health care providers), in favour of promoting the PA guidelines. Future work should examine if rebranding these guidelines as screen time guidelines could improve awareness.

\section{Limitations}

CAPL is a large, multi-site study that provides a comprehensive examination on a large group of children. ISCOLE-CAPL provides the added benefit of directly measured habitual activity, and a more comprehensive set of surveys, including parental and household characteristics. Adding the CAPL, and ISCOLE-CAPL samples together provides the largest, and most up-to-date examination of children's awareness of Canadian PA and ST guidelines. Researchers from CAPL, and ISCOLE-CAPL received comprehensive training and were provided consistent feedback before and during survey administration to minimize potential bias. Additionally, accelerometers have been shown to be a valid tool to measure activity of all levels of intensity. However, it is well understood that a hip-placed monitor is less effective in distinguishing sedentary postures, and cannot accurately capture upper body movements, cycling, or activities when the monitor is removed (e.g., swimming). For ISCOLE, algorithms were used to distinguish sleep from SED. The algorithms have been validated and published, ${ }^{13}$ and accelerometer cut points for SED have high sensitivity, ${ }^{17}$ but there is always the possibility of misclassification. It is 
important to note that this study relies predominantly on information from child-report questionnaires. Specifically, information related to guideline awareness was obtained via prompted questioning and may be biased. The child may also have simply chosen the correct response by chance. To provide context for the children's responses, it would have been beneficial for teachers and parents to provide information on their knowledge of PA guidelines and habitual levels of PA as well. Finally, it is important to note that this study is limited to a small geographic region, so generalizability of the results may be limited.

\section{Conclusions}

This study provides encouraging results about children's knowledge of current PA guidelines. It is less encouraging that relatively few children are able to correctly identify ST guidelines, but provides areas of opportunity to improve messaging associated with the guidelines. This research also shows that children with more favourable measures of activity (i.e., higher levels of PA, lower levels of SED and ST), had better self-perceptions of physical activity. Guideline awareness provides important information on overall effectiveness of knowledge dissemination among the general public. Naturally, it will take some time for the guidelines to make their way in to mainstream educational material. It is important to continue to monitor progress and

develop new and innovative ways (e.g., Twitter, Facebook ${ }^{27}$ ) to disseminate information about the guidelines to health care providers, and the general public.

\section{IMPLICATIONS FOR SCHOOL HEALTH}


Year after year we are seeing that children are not meeting physical activity guidelines, engaging in substantial amounts of sedentary behaviour, and accumulating excessive amounts of ST. ${ }^{28}$ However, children typically engage in more PA, and less sedentary behaviour during weekdays compared to weekends. ${ }^{29}$ Suggesting that the school day may provide a good opportunity to teach children about the importance of healthy lifestyle behaviours including improving self-perceptions of PA, and increase their knowledge about public health guidelines. In fact, in 2015, the Canadian Physical Activity and Sedentary Behaviour Guidelines were included in the Ontario Health and Physical Education Curriculum. ${ }^{30}$ This provides educators with a dedicated time to have these discussions with students, and will hopefully lead to increased awareness. This also means that researchers will need to make concerted efforts to continue to monitor and evaluate awareness of the guidelines while providing parents, caregivers, and teachers the tools they need to disseminate the information.

\section{Human Subjects Approval Statement}

This project was approved by the Research Ethics Board at the Children's Hospital of Eastern Ontario, the University of Ottawa, and the participating school boards. Written informed parental consent and child assent were obtained for all participants.

\section{ACKNOWLEDGEMENTS}

We thank Priscilla Bélanger, Claire Francis, Jessica McNeil, Hadiza Amedu-Ode and Nina Azoug-Boneault for their role in data collection for the Canadian site of ISCOLE and CAPL data collection. We also thank the Coordinating Center of ISCOLE in Baton 
Rouge, Louisiana, specifically Drs. Peter Katzmarzyk and Timothy Church. We also thank the study participants along with their parents, teachers and school principals for their involvement in the study. ISCOLE was funded by the Coca-Cola Company. The funder had no role in study design, data collection and analysis, decision to publish or preparation of this manuscript. 


\section{REFERENCES}

1. Tremblay MS, Warburton DER, Janssen I, Paterson DH, Latimer AE, Rhodes RE, Kho ME, Hicks A, LeBlanc AG, Zehr L, Murumets K, Duggan M. New Canadian Physical Activity Guidelines. Appl. Physiol. Nutr. Metab. 2011;36(1):36-46. doi:10.1139/H11-009.

2. Tremblay MS, LeBlanc AG, Janssen I, Kho ME, Hicks A, Murumets K, Colley RC, Duggan M. Canadian Sedentary Behaviour Guidelines for Children and Youth. Appl. Physiol. Nutr. Metab. 2011;36(1):59-64. doi:10.1139/H11-012.

3. Tremblay MS, Shephard RJ, Brawley LR, Cameron C, Craig CL, Duggan M, Esliger DW, Hearst W, Hicks A, Janssen I, Katzmarzyk PT, Latimer AE, Martin Ginis KA, McGuire A, Paterson DH, Sharratt M, Spence JC, Timmons B, Warburton D, Young TK, Zehr L. Physical activity guidelines and guides for Canadians: facts and future. Appl. Physiol. Nutr. Metab. 2007;32(S2E):S218-S224. doi:10.1139/H07-125.

4. Janssen I, LeBlanc AG. Systematic review of the health benefits of physical activity and fitness in school-aged children and youth. Int. J. Behav. Nutr. Phys. Act. 2010;7(1):40. doi:10.1186/1479-5868-7-40.

5. Tremblay MS, LeBlanc AG, Kho ME, Saunders TJ, Larouche R, Colley RC, Goldfield G, Gorber SC. Systematic review of sedentary behaviour and health indicators in schoolaged children and youth. Int. J. Behav. Nutr. Phys. Act. 2011;8(1):98. doi:10.1186/14795868-8-98.

6. LeBlanc AG, Berry T, Deshpande S, Duggan M, Faulkner G, Latimer-Cheung AE, O’Reilly N, Rhodes RE, Spence JC, Tremblay MS. Knowledge and awareness of Canadian 
Physical Activity and Sedentary Behaviour Guidelines: a synthesis of existing evidence. Appl. Physiol. Nutr. Metab. 2015;40(7):716-724. doi:10.1139/apnm-2014-0464.

7. Carson V, LeBlanc CM, Moerau E, Tremblay MS. Paediatricians' awareness of, agreement with and use of the new Canadian Physical Activity and Sedentary Behaviour Guidelines for children and youth zero to 17 years of age. Paediatr. Child Health 2013;18(10):538-542.

8. LeBlanc AG, Berry T, Deshpande S, Duggan M, Faulkner G, Latimer-Cheung A, O’Reilly N, Rhodes R, Spence JC, Tremblay MS. Knowledge, awareness, and uptake of Canadian physical activity and sedentary behaviour guidelines. Appl. Physiol. Nutr. Metab. 2014;39(S1):27.

9. Longmuir P. Understanding the physical literacy journal of children: the Canadian Assessment of Physical Literacy. Int. Counc. Sport Sci. Phys. Educ. 2013;65:276-282.

10. Longmuir P, Boyer C, Lloyd M, Yang Y, Boiarskaia E, Zhu W, Tremblay MS. The Canadian Assessment of Physical Literacy: methods for children in grades 4 to 6 ( 8 to 12 years). BMC Public Health 2015;15:767.

11. Katzmarzyk PT, Barreira TV, Broyles ST, Champagne CM, Chaput J-P, Fogelholm M, Hu G, Johnson WD, Kuriyan R, Kurpad A, Lambert EV, Maher C, Maia J, Matsudo V, Olds T, Onywera V, Sarmiento OL, Standage M, Tremblay MS, Tudor-Locke C, Zhao P, Church TS. The International Study of Childhood Obesity, Lifestyle and the Environment (ISCOLE): design and methods. BMC Public Health 2013;13(1):900. doi:10.1186/14712458-13-900. 
12. Cenre for Disease Control and Prevention. Assessing your weight: children's BMI tool for school. Child Teen BMI Calc. 2014. Available at:

http://www.cdc.gov/healthyweight/assessing/bmi/childrens_bmi/tool_for_schools.html

13. Barreira TV, Schuna JM, Mire EF, Katzmarzyk PT, Chaput J-P, Leduc G, Tudor-

Locke C. Identifying Children's Nocturnal Sleep Using 24-h Waist Accelerometry. Med.

Sci. Sports Exerc. September 2014. doi:10.1249/MSS.0000000000000486.

14. Tudor-Locke C, Mire EF, Dentro KN, Barreira TV, Schuna JM, Zhao P, Tremblay

MS, Standage M, Sarmiento OL, Onywera V, Olds T, Matsudo V, Maia J, Maher C, Lambert EV, Kurpad A, Kuriyan R, Hu G, Fogelholm M, Chaput J-P, Church TS, Katzmarzyk PT. A model for presenting accelerometer paradata in large studies: ISCOLE. Int. J. Behav. Nutr. Phys. Act. 2015;12(1):52. doi:10.1186/s12966-015-0213-5.

15. Trost SG, Loprinzi PD, Moore R, Pfeiffer KA. Comparison of Accelerometer Cut Points for Predicting Activity Intensity in Youth: Med. Sci. Sports Exerc. 2011;43(7):13601368. doi:10.1249/MSS.0b013e318206476e.

16. Colley RC, Connor Gorber S, Tremblay MS. Quality control and data reduction procedures for accelerometry-derived measures of physical activity. Health Rep. 2010;21(1):63-69.

17. Evenson KR, Catellier DJ, Gill K, Ondrak KS, McMurray RG. Calibration of two objective measures of physical activity for children. J. Sports Sci. 2008;26(14):1557-1565. doi:10.1080/02640410802334196.

18. Mirwald RL, Baxter-Jones ADG, Bailey DA, Beunen GP. An assessment of maturity from anthropometric measurements. Med. Sci. Sports Exerc. 2002;34(4):689-694. 
19. Sherar LB, Esliger DW, Baxter-Jones ADG, Tremblay MS. Age and Gender Differences in Youth Physical Activity: Does Physical Maturity Matter? Med. Sci. Sports Exerc. 2007;39(5):830-835. doi:10.1249/mss.0b013e3180335c3c.

20. Bell BB, Ene M, Smiley W, Schoeneberger JA. A Multilevel Model Primer Using SAS PROC MIXED. University of South Carolina; 2013.

21. Kenward MG, Roger JH. Small Sample Inference for Fixed Effects from Restricted Maximum Likelihood. Biometrics 1997;53(3):983. doi:10.2307/2533558.

22. Craney TA, Surles JG. Model-Dependent Variance Inflation Factor Cutoff Values. Qual. Eng. 2002;14(3):391.

23. Saunders TJ, Chaput J-P, Goldfield GS, Colley RC, Kenny GP, Doucet E, Tremblay MS. Prolonged sitting and markers of cardiometabolic disease risk in children and youth: a randomized crossover study. Metabolism. 2013;62(10):1423-1428. doi:10.1016/j.metabol.2013.05.010.

24. Debastiani SD, Carroll DD, Cunningham M, Lee S, Fulton J. Awareness and Knowledge of the Youth 2008 Physical Activity Guidelines for Americans. J. Phys. Act. Health 2013;11(3):495-501.

25. Kay MC, Carroll DD, Carlson SA, Fulton JE. Awareness and Knowledge of the 2008 Physical Activity Guidelines for Americans. J. Phys. Act. Health 2014;11(4):693-698. doi:10.1123/jpah.2012-0171.

26. Sedentary Behaviour Research Network. Letter to the Editor: Standardized use of the terms "sedentary" and "sedentary behaviours." Appl. Physiol. Nutr. Metab. 2012;37(3):540-542. doi:10.1139/h2012-024. 
27. Lefebvre RC, Bornkessel AS. Digital Social Networks and Health. Circulation 2013;127(17):1829-1836. doi:10.1161/CIRCULATIONAHA.112.000897.

28. ParticipACTION. The Biggest Risk Is Keeping Our Kids Indoors. The 2015 Report Card on Physical Activity for Children and Youth. Toronto, Ontario; 2015.

29. Treuth MS, Catellier DJ, Schmitz KH, Pate RR, Elder JP, McMurray RG, Blew RM, Yang S, Webber L. Weekend and Weekday Patterns of Physical Activity in Overweight and Normal-weight Adolescent Girls. Obesity 2007;15(7):1782-1788.

doi:10.1038/oby.2007.212.

30. Ministry of Education. Health and Physical Education: The Ontario Curriculum Grades 1-8. Ontario Public Service; 2015. Available at: www.edu.gov.on.ca. 


\section{Table 1: Descriptive Participant Characteristics}

\begin{tabular}{|c|c|c|c|}
\hline CAPL sample & Total $(n=787)$ & Boys $(n=367)$ & Girls $(n=420)$ \\
\hline Age (years) & $10.0(1.3)$ & $10.0(1.3)$ & $10.0(1.3)$ \\
\hline Waist circumference $(\mathrm{cm})$ & $66.0(10.4)$ & $66.1(10.0)$ & $65.9(10.8)$ \\
\hline BMI z-score & $0.49(1.04)$ & $0.56(1.00)$ & $0.42(10.10)$ \\
\hline ISCOLE-CAPL subsample & Total $(n=223)$ & Boys $(n=85)$ & Girls (n=138) \\
\hline Age (years) & $10.0(0.34)$ & $10.1(0.34)$ & $10.0(0.34)$ \\
\hline Waist circumference $(\mathrm{cm})$ & $61.3(6.7)$ & $62.6(6.7)$ & $60.4(6.7)^{+}$ \\
\hline BMI z-score & $0.22(0.98)$ & $0.41(0.97)$ & $0.10(0.98)^{\dagger}$ \\
\hline Maturity offset & $-1.9(0.88)$ & $-2.84(0.46)$ & $-1.37(0.54)^{*}$ \\
\hline \multicolumn{4}{|l|}{ Household income $(n, \%)$} \\
\hline Less than $\$ 15,000$ & $2(0.9)$ & $1(1.2)$ & $2(1.5)$ \\
\hline$\$ 15,000--59,999$ & $20(9.2)$ & $2(2.4)$ & $17(12.5)$ \\
\hline$\$ 60,000-139,999$ & $97(44.5)$ & $34(40.2)$ & $64(57.1)$ \\
\hline$\$ 140,000$ and above & $99(45.4)$ & $46(56.1)$ & $53(39.0)$ \\
\hline \multicolumn{4}{|l|}{ Parental education ${ }^{\ddagger}$} \\
\hline Mother education & $195(88.2 \%)$ & $74(89.2 \%)$ & $121(87.7 \%)$ \\
\hline Father education & $189(85.5 \%)$ & $73(88.0 \%)$ & $116(84.1 \%)$ \\
\hline \multicolumn{4}{|c|}{ Accelerometer variables (min/day) } \\
\hline Light intensity & $301.9(43.0)$ & $305.6(40.1)$ & $299.6(44.7)$ \\
\hline MVPA & $58.1(19.6)$ & $66.3(19.4)$ & $53.0(18.0)^{*}$ \\
\hline Sedentary behaviour & $508.2(60.1)$ & $505.3(61.1)$ & $510.1(59.7)$ \\
\hline \multicolumn{4}{|c|}{ Self-report screen time score (hours/day) } \\
\hline Total screen time & $2.4(1.6)$ & $2.6(1.2)$ & $2.3(1.7)$ \\
\hline Computer/video game & $1.3(1.0)$ & $1.4(1.0)$ & $1.0(1.0)^{\dagger}$ \\
\hline Television & $1.3(0.9)$ & $1.2(0.7)$ & $1.3(1.1)$ \\
\hline
\end{tabular}

All variables presented as mean (SD) unless otherwise noted. Statistical significance between boys and

girls was assessed with unpaired t-test for continuous variables and chi-square tests for household income and parental education.

BMI: Body Mass Index; MVPA: moderate- to vigorous-intensity physical activity

$* \mathrm{p}<0.01, \mathrm{tp}<0.0001$

‡Mother and father education is presented as percentage who have self-reported that they have obtained a high school diploma or higher 
Table 2: Responses to the Knowledge and Understanding of Physical Activity

Questionnaire in Boys and Girls (total sample $n=1,010$ )

\begin{tabular}{|c|c|c|c|}
\hline & Total $(n, \%)$ & Boys $(n, \%)$ & Girls $(n, \%)$ \\
\hline \multicolumn{4}{|c|}{ Knowledge of guidelines } \\
\hline \multicolumn{4}{|c|}{ How many minutes of MVPA should you get each day? } \\
\hline 10 minutes & $34(3.5)$ & $15(3.5)$ & $19(3.5)$ \\
\hline 20 minutes & $52(5.3)$ & $23(5.3)$ & $29(5.4)$ \\
\hline 30 minutes & $192(19.6)$ & $82(19.0)$ & $110(20.3)$ \\
\hline 60 minutes or 1 hour & $700(71.6)$ & $312(72.3)$ & $383(70.8)$ \\
\hline \multicolumn{4}{|c|}{$\begin{array}{l}\text { What is the most time that children should look at a } \\
\text { screen each day? }{ }^{\ddagger}\end{array}$} \\
\hline 30 minutes & $373(38.2)$ & $146(34.0)$ & $224(41.3)$ \\
\hline 60 minutes or 1 hour & $305(31.2)$ & $134(31.2)$ & $170(31.4)$ \\
\hline 2 hours & $195(20.0)$ & $98(22.8)$ & $96(17.7)^{*}$ \\
\hline 4 hours & $104(10.6)$ & $52(12.1)$ & $52(9.6)$ \\
\hline \multicolumn{4}{|c|}{ Perceptions of physical activity } \\
\hline \multicolumn{4}{|c|}{$\begin{array}{l}\text { If you wanted to get better at a sport skill like kicking and } \\
\text { catching a ball, what would be the best thing to do? }\end{array}$} \\
\hline Read a book & $19(2.0)$ & $11(2.6)$ & $8(1.5)$ \\
\hline Wait until you get older & $13(1.4)$ & $6(1.4)$ & $7(1.3)$ \\
\hline Try exercising and being more active & $611(63.7)$ & $252(59.7)$ & $356(66.8)$ \\
\hline Take a lesson & $317(33.0)$ & $153(36.3)$ & $162(30.4)$ \\
\hline \multicolumn{4}{|c|}{$\begin{array}{l}\text { If you wanted to get in better shape, what would be the } \\
\text { best thing to do? }\end{array}$} \\
\hline Read a book & $10(1.0)$ & $4(2.0)$ & $6(1.1)$ \\
\hline Wait until you get older & $21(2.2)$ & $12(2.8)$ & $9(1.7)$ \\
\hline Try exercising or being more active & $845(88.2)$ & $366(86.7)$ & $475(89.5)$ \\
\hline Take a lesson & $82(8.6)$ & $4(9.5)$ & $41(7.7)$ \\
\hline \multicolumn{4}{|c|}{$\begin{array}{l}\text { If you were allowed to pick what you do after school, } \\
\text { which activity would you pick?§ }\end{array}$} \\
\hline Sedentary pursuit & $294(31.1)$ & $144(35.2)$ & $150(28.3)$ \\
\hline Active pursuit & $650(68.9)$ & $265(64.8)$ & $380(71.7)$ \\
\hline \multicolumn{4}{|c|}{ Compared to other kids your age, how active are you? } \\
\hline A lot less active (1-3) & $48(4.9)$ & $18(4.2)$ & $30(5.6)$ \\
\hline Same (4-7) & $512(53.2)$ & $206(47.6)$ & $313(57.8)$ \\
\hline A lot more active $(8-10)$ & $410(41.8)$ & $209(48.3)$ & $198(36.6) \S$ \\
\hline \multicolumn{4}{|c|}{$\begin{array}{l}\text { Compared to other kids your age, how good are you at } \\
\text { sports or skills? }\end{array}$} \\
\hline Others are better (1-3) & $95(9.7)$ & $40(9.3)$ & $55(10.2)$ \\
\hline Same (4-7) & $512(52.3)$ & $179(41.4)$ & $332(61.3)$ \\
\hline I'm a lot better (8-10) & $372(38.0)$ & $213(49.3)$ & $155(28.6)^{\dagger}$ \\
\hline
\end{tabular}

Significance between boys and girls was assessed by chi-squared test: ${ }^{*} p<0.05,+p<0.0001$

$\neq$ This includes all television, video game, and computer use. Guidelines recommend limiting recreational screen time to no more than 2 hours per day ${ }^{2}$.

$\S$ Sedentary: play video/computer games, read, homework, chat with friends online, watch TV; active: play with friends at the playground, go to my sports team's practice, walk my dog MVPA: moderate- to vigorous-intensity physical activity. 
1 Table 3: Associations Between Habitual Activity and Perceptions of Physical Activity (ISCOLE-CAPL subsample, $n=202$ )

\begin{tabular}{|c|c|c|c|c|c|c|c|c|c|}
\hline & \multicolumn{3}{|c|}{ MVPA (min/day) } & \multicolumn{3}{|c|}{ SED (hour/day) } & \multicolumn{3}{|c|}{ ST Score } \\
\hline & $\beta$ & SE & $p$ & $\beta$ & SE & $p$ & $\beta$ & SE & $p$ \\
\hline \multicolumn{10}{|c|}{$\begin{array}{l}\text { If you wanted to get better at a } \\
\text { sport skill like kicking and } \\
\text { catching a ball, what would be } \\
\text { the best thing to do? }\end{array}$} \\
\hline Model 1 & 0.89 & 3.02 & 0.7680 & -0.08 & 0.15 & 0.5820 & -0.003 & 0.07 & 0.9709 \\
\hline Model 2 & 4.38 & 2.86 & 0.1273 & -0.21 & 0.15 & 0.1519 & 0.02 & 0.07 & 0.7924 \\
\hline \multicolumn{10}{|c|}{$\begin{array}{l}\text { If you wanted to get in better } \\
\text { shape, what would be the best } \\
\text { thing to do? }\end{array}$} \\
\hline Model 1 & -3.20 & 6.49 & 0.6226 & -0.14 & 0.32 & 0.6769 & 0.27 & 0.14 & 0.0534 \\
\hline Model 2 & -9.33 & 6.08 & 0.1271 & -0.01 & 0.31 & 0.9740 & 0.28 & 0.14 & 0.0487 \\
\hline \multicolumn{10}{|c|}{$\begin{array}{l}\text { If you were allowed to pick what } \\
\text { you do after school, which } \\
\text { activity would you pick? }\end{array}$} \\
\hline Model 1 & -2.45 & 3.30 & 0.4592 & 0.23 & 0.17 & 0.1599 & 0.17 & 0.08 & 0.0227 \\
\hline Model 2 & -3.12 & 3.13 & 0.3201 & 0.23 & 0.16 & 0.1480 & 0.13 & 0.07 & 0.0744 \\
\hline \multicolumn{10}{|c|}{$\begin{array}{l}\text { Compared to other kids your } \\
\text { age, how active are you? }\end{array}$} \\
\hline Model 1 & -21.64 & 5.73 & 0.0002 & 0.56 & 0.30 & 0.0599 & 0.43 & 0.14 & 0.0011 \\
\hline Model 2 & -19.26 & 5.44 & 0.0005 & 0.41 & 0.14 & 0.1583 & 0.42 & 0.13 & 0.0013 \\
\hline \multicolumn{10}{|c|}{$\begin{array}{l}\text { Compared to other kids your } \\
\text { age, how good are you at sports } \\
\text { or skills? }\end{array}$} \\
\hline Model 1 & -12.74 & 5.89 & 0.0318 & 0.27 & 0.30 & 0.3634 & 0.33 & 0.14 & 0.0151 \\
\hline Model 2 & -9.01 & 5.62 & 0.1089 & 0.20 & 0.29 & 0.4836 & 0.33 & 0.13 & 0.0131 \\
\hline
\end{tabular}

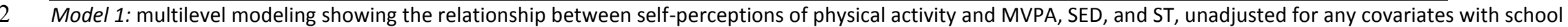
as a random effect.

Model 2: multi-level modeling showing the relationship between self-perceptions of physical activity and MVPA, SED, and ST adjusted for sex, waist circumference, maturity offset, and highest parental education with school as a random effect.

MVPA: moderate- to vigorous-intensity physical activity; SE: standard error; SED: total sedentary time; ST: screen time (log transformed). Bolded cells represent significant associations. 


\section{Figure legends}

\section{Figure 1}

Title: Average moderate- to vigorous-intensity physical activity (MVPA), total sedentary time, and knowledge of physical activity (A) and screen time guidelines (B) in ISCOLECAPL subsample $(n=202)$.

Legend: Data are presented as means \pm standard error (SE). Participant's answer to knowledge of physical activity and screen time guidelines were re-coded as correct (white bars), or incorrect (grey bars). Multi-level models, adjusted for sex, maturity offset, waist circumference, and highest parental education were used to determine associations between physical activity $(\beta=-3.36, \mathrm{SE}=2.95, p=0.2555)$, and sedentary behaviour $(\beta=0.26, \mathrm{SE}=0.16, p=0.8637)$, and knowledge of the guidelines. 

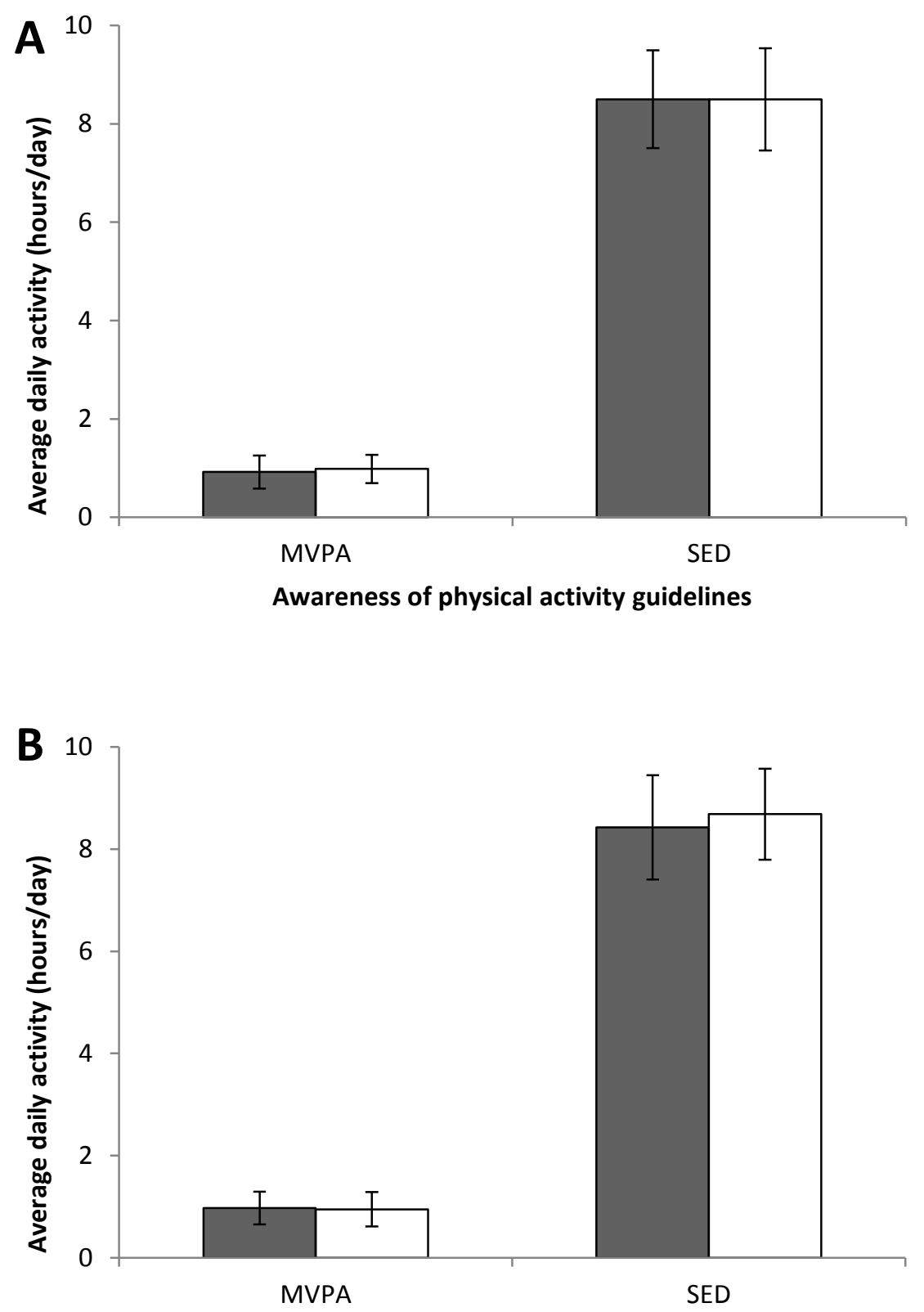

Awareness of screen time guidelines 
4.2 Correlates of objectively measured sedentary time and self-reported screen time in Canadian children

This manuscript has been written and formatted to adhere to the specifications for submission to the International Journal of Behavioural Nutrition and Physical Activity. This article has been published and can be found here:

http://www.ijbnpa.org/content/12/1/38/ 


\title{
Correlates of objectively measured sedentary time and self-reported screen time in Canadian children
}

Allana G LeBlanc ${ }^{1,2 *}$, Stephanie T Broyles ${ }^{3}$, Jean-Philippe Chaput ${ }^{1,2,4,5}$, Geneviève Leduc' ${ }^{1}$ Charles Boyer ${ }^{1}$, Michael M Borghese ${ }^{1,5}$ and Mark S Tremblay ${ }^{1,2,4,5}$

\begin{abstract}
Background: Demographic, family, and home characteristics play an important role in determining childhood sedentary behaviour. The objective of this paper was to identify correlates of total sedentary time (SED) and correlates of self-reported screen time (ST) in Canadian children.

Methods: Child- and parent-reported household, socio-demographic, behavioural, and diet related data were collected; directly measured anthropometric and accelerometer data were also collected for each child. Participants with complete demographic, anthropometric, and either SED ( $n=524,41 \%$ boys) or ST ( $n=567,42 \%$ boys) data from the Canadian site of the International Study of Childhood Obesity Lifestyle and the Environment (ISCOLE) were included in analysis. Sixteen potential correlates of SED and ST were examined using multilevel general linear models, adjusting for sex, ethnicity, number of siblings, and socio-economic status. All explanatory variables moderately associated $(p<0.10)$ with SED and/or $S T$ in univariate analyses were included in the final, fully-adjusted models. Variables that remained significant in the final models $(p<0.05)$ were considered correlates of SED and/or ST.

Results: Children averaged 8.5 hours of daily SED; no differences in total SED, or total ST were seen between girls and boys, but boys reported significantly more video game/computer usage than girls. Boys also had higher waist circumference and BMI z-scores than girls. In the final models, waist circumference and number of TVs in the home were the only common correlates of both SED and ST. SED was also negatively associated with sleep duration. ST was also positively associated with mother's weight status, father's education, and unhealthy eating pattern score and negatively associated with healthy eating pattern score, and weekend breakfast consumption. Few common correlates existed between boys and girls.

Conclusion: Several factors were identified as correlates of SED and/or of ST in Canadian children; however, few correlates were common for both SED and ST, and for both boys and girls. This suggests that a single strategy to reduce SED and ST is unlikely to be effective. Future work should examine a variety of other, non-screen based sedentary behaviours and their potential correlates in the hopes of creating tailored public health messages to reduce SED and ST in both boys, and girls.

Keywords: Sedentary behaviour, Television viewing, Pediatric
\end{abstract}

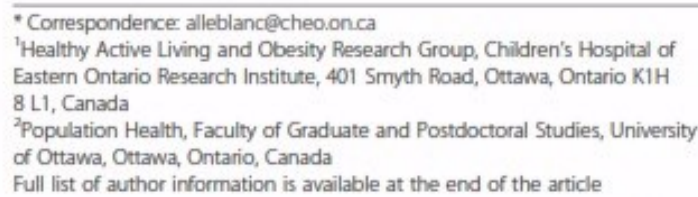




\section{Introduction}

Sedentary time (SED) is characterized by waking behaviours that require little energy expenditure (i.e. $\leq 1.5 \mathrm{METs}$ ) and that occur in a sitting or reclined position [1]. SED can be further classified by a variety of sedentary behaviours such as reading, playing quietly, watching television (TV), or using the computer. SED should be thought of as a separate and distinct behaviour from physical activity and not simply the failure to meet prescribed physical activity guidelines [2]. To help guide parents and caregivers, the Canadian Society for Exercise Physiology developed Canadian Sedentary Behaviour Guidelines for Children and Youth [3]. They provide a general recommendation for children and youth to minimize the time they spend sedentary each day; furthermore, they provide specific recommendations to limit recreational screen time to no more than 2 hours per day, and to limit sedentary (i.e., motorized) transport, extended time spent sitting, and time spent indoors throughout the day [3].

Previously, research examining SED has been largely informed by parent- and child-report questionnaires, which focus primarily on specific sedentary behaviours, such as screen time (ST), rather than total daily SED. Results from a recent systematic review focusing on ST suggests that lower ST is associated with better measures of body composition, fitness, self-esteem, self-worth, pro-social behaviour, and academic achievement [4]. Now, with widespread use of accelerometers, researchers can examine total daily SED, including its effect on acute and chronic health conditions. In adults, higher total SED is associated with higher risk of cardiovascular disease, overweight/obesity, and premature mortality [5-7]. However, in children, the relationship between accelerometer measured total SED and ill-health is less evident, and possibly more complex. For example, evidence from both clinical and population based studies has shown that in children, long bouts of SED are not associated with acute elevations in cardio-metabolic health risk [8], body mass index (BMI), or waist circumference [9]. Further, previous work has shown that ST accounts for only a small proportion of SED and the appropriateness of using ST as a proxy measure for SED has been questioned [10]. Still, many studies use measures of SED and ST interchangeably, and make public health messages based on these mixed results.

A recent review by Temmel and Rhodes identified 64 studies examining correlates of sedentary behaviour however, only eleven reported on both accelerometer measured SED and self-reported ST in the same population, and none of the 64 studies included data from Canadian children [11]. Of the eleven studies examining both SED and ST, age, sex, ethnicity, socio-economic status, and day of the week (weekend versus weekday) were significant correlates of both SED and ST. Physical maturity was a correlate of SED only; whereas urban versus rural living, neighbourhood satisfaction and safety, access (e.g., to a television), sleep duration, and self-esteem were correlates of ST only. Other studies that have explored associations with ST have identified age, socio-economic status, singleparent households, ethnicity, and sex as important correlates $[12,13]$. Studies that have examined correlates of SED have shown significant associations with parental BMI, TV viewing, and computer use [14].

Identifying common correlates of SED and ST can help to inform public health strategies and messages to improve habitual behaviour in young people. The purpose of this study was to examine anthropometric, behavioural, parental, and household correlates of objectively measured SED and self-report ST in a sample of Canadian children. Analyses were grounded in the socio-ecological model for sedentary behaviour, as proposed by Owens et al. in order to understand multiple influences on behavior [15].

\section{Methods \\ Data source}

The International Study of Childhood Obesity, Lifestyle and the Environment (ISCOLE) aimed to determine the relationships between lifestyle behaviours and obesity in a multinational, cross-sectional study of 10-year-old children [16]. ISCOLE was designed to allow researchers to investigate the influence of higher-order characteristics such as behavioural settings, and the physical, social and policy environments, within and between countries [16]. Additional details on study design, participating countries, and full methodology has been published elsewhere [16]. As Canada was the first country to develop evidence-informed sedentary behaviour guidelines [3], the analyses for the present study focused on data from ISCOLE Canada. Data collection occurred in Ottawa, Canada between September 2012, and May 2013 and included 26 schools, from four school boards: English Public, French Public, English Catholic, and French Catholic. All schools within each stratum were invited to participate and the first ones to respond were included into the study with a recruitment goal of 500 participants, in agreement with the rules of our research ethics boards; the response rate was $50 \%$ (children with consent to participate divided by envelopes distributed). This project was approved by the research ethics board at the Children's Hospital of Eastern Ontario and the participating school boards. Written informed parental consent and child assent were obtained for all participants.

\section{Dependent variables}

Accelerometer measured sedentary time

The ActiGraph GT3X+ triaxial accelerometer (ActiGraph LLC, Pensacola, FL, USA) was used to objectively measure total SED. Participants wore the accelerometer, attached to an elastic belt around the waist (at the right mid-axillary line), for 7 consecutive days, 24 hours per day, removing only for aquatic activities (e.g, bathing, swimming). To increase compliance, study staff instructed children how to 
wear the accelerometer during the initial in-school assessment, and conducted an in-person compliance check 2-4 days after initialization. Further, participants were contacted twice via telephone (one weekday evening and one weekday) to ensure they were wearing the device, and to address any questions.

To be included in the ISCOLE dataset, participants were required to provide at least four days of valid measurements (including at least one weekend day), with at least 10 hours of waking wear time per day $[17,18]$. Data were collected at a sampling rate of $80 \mathrm{~Hz}$, downloaded in 1-second epochs, and aggregated to 15-second epochs [18]. Total sedentary time (SED) was defined as all epochs showing $\leq 25$ counts per 15 seconds, consistent with widely used cutoffs from Evenson et al. [19] (i.e., sedentary: 0-25 counts/15 seconds, light: 26-573 counts/15 seconds, moderate-to-vigorous: $\geq 574$ counts/ 15 seconds). For analysis, total SED was treated as a continuous variable.

\section{Self-reported screen time}

Child-reported screen time was determined from a Diet and Lifestyle Questionnaire, with questions taken from the U.S. Youth Risk Behaviour Surveillance System [16,20]. Children were asked how many hours they typically watched TV, and how many hours they played video games and/or used the computer per week day, and per weekend day [16]. Responses were: 1 = I did not watch TV, 2 = Less than 1 hour, $3=1$ hour, $4=2$ hours, $5=3$ hours, $6=4$ hours, $7=5$ or more hours. Response categories were collapsed to include $1=\leq 1$ hour of TV, $2=2$ hours, $3=3$ hours, $4=$ 4 hours, and $5=\geq 5$ hours. A weighted mean score of daily screen time was then calculated as follows: [(hours of TV on weekdays $\times 5$ ) $+($ hours of TV on weekend days $\times 2$ ) + (hours of video games and computers on weekdays $\times 5$ ) + (hours of video games and computers on weekend days $\times$ 2)]/7. For analysis, this is presented as a screen time score, rather than total hours of ST since after 5 hours per day, we could not ascertain the participant's actual amount of ST. Self-report methods of quantifying ST have been reported to have acceptable reliability and validity in children [21]. After testing for normality, ST score was log-transformed for analysis and treated as a continuous variable.

\section{Potential correlates}

\section{Anthropometric and biological variables}

Anthropometric data (i.e., height, weight, waist circumference, percent body fat) were directly measured by trained ISCOLE data collectors during the in-school visit according to standardized procedures [16]. Height was measured to the nearest $0.1 \mathrm{~cm}$ using a Seca 213 portable stadiometer (Hamburg, Germany). Weight (to the nearest $0.1 \mathrm{~kg}$ ) and body fat percentage (to the nearest $0.1 \%$ ) were measured using a portable Tanita SC-240 Body Composition Analyzer
(Arlington Heights, IL, USA). Participants were asked to remove heavy clothing, and objects from their pockets before stepping on the scale. The Tanita SC-240 has shown acceptable accuracy for estimating body fat percentage when compared with dual-energy X-ray absorptiometry, supporting its use in field-based studies [22]. Body mass index (BMI) was calculated $\left(\mathrm{kg} / \mathrm{m}^{2}\right)$, and BMI z-score, based on age and gender, was determined using the Centers for Disease Control and Prevention (CDC) growth charts [23] for all participants. Waist circumference was measured using a non-elastic anthropometric tape after a normal exhalation to the nearest $0.1 \mathrm{~cm}$, at the mid-point between the top of the iliac crest and the bottom of the last floating rib. Children and parents were also asked about the child's age, sex, birth country and ethnicity in the ISCOLE Diet and Lifestyle Questionnaire and the Demographic and Family History Questionnaire.

\section{Behavioural characteristics}

All children completed the ISCOLE Diet and Lifestyle Questionnaire, which included a compilation of validated questions from several questionnaires [16]. Children completed a Food Frequency Questionnaire (FFQ) adapted from the Health Behaviours in School-age Children (HBSC) study [24], which asked how often participants consumed 23 food items in a usual week. To identify existing dietary patterns among the study population, principal components analyses were carried out using the FFQ food groups as input variables (excluding fruit juices due to low validity) (unpublished analysis). Eigenvalues and a scree plot analysis were used as the criteria for deciding the number of factors extracted. The two criteria lead to similar conclusions and two factors were eventually chosen for each analysis. The factors were then rotated with an orthogonal varimax transformation to force non-correlation of the factors and to enhance the interpretation. Two factors were included in this manuscript as exposure variables: "unhealthy eating pattern score" (e.g., hamburgers, soft drinks, fried food; higher score means worse eating pattern) and "healthy eating pattern score" (e.g., vegetables and fruits; higher score means better eating pattern).

Children were asked how they got to school most days (e.g., walking, car), and these responses were re-coded as active or inactive transport. Child-reported breakfast consumption (weekday and weekend frequency), and sleep (quality and quantity) were also assessed as potential correlates. Physical activity was captured via self-report questionnaire to determine how many days per week children were active for at least 60 minutes.

\section{Parent and home environment}

The Demographic and Family History Questionnaire and the ISCOLE neighbourhood and home environment 
questionnaires were sent home with the child at the same time as the parent-consent forms for the parents to fill out (i.e. prior to child assessment) [16]. Demographic questionnaires completed by the parents captured additional information about the participating number of siblings, household income ( 8 options ranging from $<\$ 14,999$, to $>$ $\$ 140,000$ or more), parental education (ranging from "less than high school" to "post graduate degree"), parental-report weight and height (used to calculate parental weight status), number of TVs in the home, and presence of a TV in the child's bedroom. See Table 1 for additional details on response categories and additional details on measurement and analysis of all potential correlates.

\section{Statistical analysis}

Statistical analyses were conducted using SAS 9.4 (SAS Institute Incorporated, North Carolina, USA). Descriptive information (mean, standard deviation) was calculated for demographic and anthropometric characteristics of all participants and their parents. Unpaired t-tests and chisquared tests were run to determine differences between boys and girls.

Multilevel general linear models (PROC MIXED), including school as a random effect, were used to determine correlates of SED and ST. Multilevel models properly account for the hierarchical nature of the data. Potential correlates were first included in univariate models; variables that were significant, or marginally significant $(\mathrm{p}<0.10)$, were subsequently included in domain-specific models similar to those outlined in the social ecological model proposed by Owen et al. for sedentary behaviour [15] (i.e., demographic/biological characteristics, family characteristics, home environment characteristics, or behavioural characteristics). Sex and ethnicity were included as covariates for all univariate models. Variables that were marginally significantly $(\mathrm{p}<$ $0.10)$ correlated with SED or ST in the domain-specific models were included in the final model. Variables that remained significant $(\mathrm{p}<0.05)$ in the final model were considered correlates of SED and/or ST. Sex, ethnicity, number of siblings, and household income were used as covariates for all multivariable models. These covariates were selected based on the plausibility of their potential confounding effect and because of their known associations with sedentary behaviour. The Kenward-Roger approximation (DDFM = KR) was used to calculate degrees of freedom [25]. Analyses were conducted separately for the total sample, boys, and girls. Multicollinearity was tested using tolerance and variance inflation factors, and results indicated no issues with multicollinearity [26].

\section{Results}

\section{Descriptive characteristics}

In total, 567 (mean age $=10.0,42.3 \%$ boys) participants provided complete data and were included in analysis.
Participants were from the English Public ( $n=393 ; 69.3 \%$ of total sample), French Public ( $n=60 ; 10.6 \%$ of total sample), English Catholic ( $\mathrm{n}=75 ; 13.2 \%$ of total sample) and French Catholic $(\mathrm{n}=39 ; 6.8 \%$ of total sample) School Boards. Participants were born in 35 different countries, with the majority ( $88.9 \%$ ) being born in Canada, and declaring themselves as "white/Caucasian" (66.6\%). The majority of children had either one (51.3\%) or two (23.7\%) siblings. Table 2 shows the frequency and distribution of potential correlates of sedentary behaviour between boys and girls.

The majority of mothers were normal weight $(64.8 \%)$ and had above a high school education (84.7\%); the majority of fathers were overweight or obese $(56.8 \%)$ and had above a high school education (81.3\%). Most parents reported having two or more TVs in their house $(96.2 \%)$ with the remainder reporting having either no TV, or one TV; the majority subscribed to cable + premium channels (39.6\%) with cable internet service (59.1\%). No differences were seen in parental characteristics between boys and girls.

Most children ate breakfast every weekday (82.9\%) and every weekend day $(89.8 \%)$. The majority of children reported that they had "very good" or "fairly good" sleep quality $(91.4 \%)$ and sleep quantity $(90.8 \%)$. On average, ISCOLE participants accumulated an average of 511.4 (63.1) minutes of total daily SED (approximately 8.5 hours) and had a ST score of 2.8 (1.8) (approximately 2.8 hours of average daily total TV, computer, and video game use). Total ST did not differ between boys and girls, but boys reported playing more video games than girls.

\section{Univariate analyses}

The results of the univariate regression analyses are presented in Tables 3 and 4 . Of the 16 potential behavioural, parental, and home correlates, 5 were significantly associated with SED in the total sample (4 for boys, and 7 for girls) and 11 were significantly associated with ST in the total sample (10 for boys, and 9 for girls).

\section{Multivariate analyses \\ Total sedentary time}

Results of the multivariate regression models for correlates of total SED are presented in Table 5. In the total sample, and for boys alone, SED was positively associated with waist circumference, and number of TVs in the home; SED was negatively associated with sleep quantity. For girls, SED was positively associated with waist circumference, and household automobile ownership.

\section{Screen time}

Results of multivariate regression models for correlates of self-reported ST are presented in Table 5. In the whole sample, ST was positively associated with waist circumference, mother's weight status (classified overweight or obese), father's education (greater than high school), number of TVs 
Table 1 Potential correlates of objectively measured sedentary time and self-reported sedentary behaviour

\begin{tabular}{|c|c|c|}
\hline Variable & Measurement method & Use in analysis \\
\hline \multicolumn{3}{|l|}{ Anthropometric } \\
\hline Sex & $\begin{array}{l}\text { Parent-report: Demographic and Family } \\
\text { History Questionnaire }\end{array}$ & Binary variable: male, fernale (used as a covariate) \\
\hline BMI & $\begin{array}{l}\text { Directly measured height and weight, } \\
\text { calculated using CDC cut-points [23] }\end{array}$ & Ordered categorical: underweight, normal weight, overweight, obese \\
\hline Percent body fat & Directly measured using Tanita & Continuous \\
\hline Waist circumference & Directly measured by ISCOLE researcher & Continuous \\
\hline Child ethnicity & $\begin{array}{l}\text { Parent-report: Demographic and Family } \\
\text { History Questionnaire }\end{array}$ & $\begin{array}{l}\text { Categorical: white, African American, Asian, First Nations, East Indian, don't } \\
\text { know, other (used as a covariate) }\end{array}$ \\
\hline \multicolumn{3}{|l|}{ Behavioural characteristics } \\
\hline $\begin{array}{l}\text { Healthy and unhealthy } \\
\text { eating scores }\end{array}$ & $\begin{array}{l}\text { Child-report food frequency questionnaire: } \\
\text { ISCOLE Diet and Lifestyle Questionnaire }\end{array}$ & $\begin{array}{l}\text { Continuous: Continuous: obtained from a principal component analysis } \\
\text { derived from a } 23 \text {-item food frequency questionnaire }\end{array}$ \\
\hline $\begin{array}{l}\text { Breakfast consumption } \\
\text { (weekend, and weekday) }\end{array}$ & $\begin{array}{l}\text { Child-report: ISCOLE Diet and Lifestyle } \\
\text { Questionnaire }\end{array}$ & $\begin{array}{l}\text { Re-coded as dichotomous: those who ate breakfast at least once per } \\
\text { weekday (versus never) and those who at breakfast at least weekend day } \\
\text { (versus never). }\end{array}$ \\
\hline $\begin{array}{l}\text { Commute to school } \\
\text { (main part of journey) }\end{array}$ & $\begin{array}{l}\text { Child-report: ISCOLE Diet and Lifestyle } \\
\text { Questionnaire }\end{array}$ & $\begin{array}{l}\text { Re-coded as dichotomous active (walking, bicycle/rollerblade/skateboard/ } \\
\text { scooter, other), and inactive (bus/train/tram/underground/boat, car/ } \\
\text { motorcycle/moped) }\end{array}$ \\
\hline Sleep (in the past week) & $\begin{array}{l}\text { Child-report: ISCOLE Diet and Lifestyle } \\
\text { Questionnaire }\end{array}$ & $\begin{array}{l}\text { Categorical: Sleep quality ( } 4 \text { responses: very good, fairly good, fairly bad, very } \\
\text { bad), sleep quantity ( } 4 \text { responses very good, fairly good, fairly bad, very bad). } \\
\text { Re-coded for analysis, collapsing very good and fairly good categories, and } \\
\text { fairly bad and very bad categories for both sleep quality and sleep quantity. }\end{array}$ \\
\hline \multirow[t]{2}{*}{ Physical activity } & $\begin{array}{l}\text { Child-report: ISCOLE Diet and Lifestyle } \\
\text { Questionnaire }\end{array}$ & $\begin{array}{l}\text { Categorical: } 8 \text { responses ( } 0 \text { days, } 1 \text { day, } 2 \text { days, } 3 \text { days, } 4 \text { days, } 5 \text { days, } 6 \text { days, } \\
7 \text { days). Was included in the model re-coded as those active more and less } \\
\text { than } 6 \text { days in the past week. }\end{array}$ \\
\hline & $\begin{array}{l}\text { Accelerometer measured minutes per day } \\
\text { of LPA and MVPA }\end{array}$ & $\begin{array}{l}\text { Accelerometer- time spent at different PA intensities using Evensen cut-points } \\
\text { [19] }\end{array}$ \\
\hline \multicolumn{3}{|l|}{ Family situation } \\
\hline Number of siblings & $\begin{array}{l}\text { Parent-report: Demographic and Family } \\
\text { History Questionnaire }\end{array}$ & Continuous (used as a covariate) \\
\hline Parental BMI & $\begin{array}{l}\text { Parent-report: Demographic and Family } \\
\text { History Questionnaire }\end{array}$ & Re-coded as dichotomous normal weight, or overweight/obese \\
\hline Parental education & $\begin{array}{l}\text { Parent-report: Demographic and Farnily } \\
\text { History Questionnaire }\end{array}$ & Re-coded as dichotomous Shigh school, and high school or higher \\
\hline $\begin{array}{l}\text { Combined household } \\
\text { income }\end{array}$ & $\begin{array}{l}\text { Parent-report: Demographic and Family } \\
\text { History Questionnaire }\end{array}$ & $\begin{array}{l}\text { Ordered categorical: } 8 \text { options ranging from }<\$ 14,999 \text {, to }>\$ 140,000 \text { or more } \\
\text { (used as covariate). In the model, income was re-coded as above/below } \\
\text { mean category. }\end{array}$ \\
\hline \multicolumn{3}{|l|}{ Home environment } \\
\hline Number of TVs in home & $\begin{array}{l}\text { Parent report ISCOLE neighbourhood and } \\
\text { home environment questionnaire }\end{array}$ & Re-coded as dichotomous: $\leq 1$, and $\geq 2$ \\
\hline \multirow[t]{2}{*}{$\begin{array}{l}\text { TV/electronics in } \\
\text { bedroom }\end{array}$} & $\begin{array}{l}\text { Child-report: ISCOLE Diet and Lifestyle } \\
\text { Questionnaire }\end{array}$ & Binary response: yes/no \\
\hline & $\begin{array}{l}\text { Parent report ISCOLE neighbourhood and } \\
\text { home environment questionnaire }\end{array}$ & \\
\hline Automobile ownership & $\begin{array}{l}\text { Parent report ISCOLE neighbourhood and } \\
\text { home environment questionnaire }\end{array}$ & $\begin{array}{l}\text { Continous: number of working automobiles owned per household. Re-coded } \\
\text { as dichotomous: }<2 \text { and } \geq 2 \text {. }\end{array}$ \\
\hline
\end{tabular}

BMI: body mass index, ISCOLE: International Study of Childhood Obesity Lifestyle and the Environment; LPA: light-intensity physical activity; MVPA: moderate- to-vigorous-intensity physical activity, PA: physical activity.

in the home, and unhealthy eating pattern score. ST was negatively associated with healthy eating pattern score and weekend breakfast consumption. In boys, ST was positively associated with number of TVs in the house, presence of a TV in the child's bedroom, and unhealthy eating pattern score score. For girls, ST was positively associated with waist circumference, mother's weight status (classified as overweight or obese), father's education (higher than high school), and unhealthy eating pattern score; negative associations were found with 
Table 2 Participant characteristics (mean (SD), unless otherwise noted)

\begin{tabular}{|c|c|c|c|}
\hline & Total $(n=567)$ & Boys $(n=239)$ & Girls $(n=328)$ \\
\hline \multicolumn{4}{|l|}{ Child characteristics } \\
\hline Age (years) & $10.0(0.4)$ & $10.1(0.4)$ & $10.0(0.4)$ \\
\hline Height $(\mathrm{cm})$ & $143.8(7.2)$ & $143.6(6.8)$ & $143.9(7.4)$ \\
\hline Weight (kg) & $38.1(9.0)$ & $38.3(9.1)$ & $379(9.1)$ \\
\hline Percent body fat & $20.5(7.4)$ & $18.7(7.2)$ & $21.9(7.3)^{*}$ \\
\hline Waist circumference (cm) & $63.0(84)$ & $64.1(8.8)$ & $622(8.0)^{* *}$ \\
\hline BMI z-score & $0.20(1.02)$ & $0.32(0.98)$ & $0.11(1.04)^{\cdots \cdots}$ \\
\hline \multicolumn{4}{|l|}{ Weight status $(n, \%)$} \\
\hline Underweight & $15(2776)$ & $2(0.896)$ & $13(4.096)$ \\
\hline Normal weight & $422(74.796)$ & $177(74.496)$ & $245(74.966)$ \\
\hline Overweight & $68(12.096)$ & $29(12.2 \% 6)$ & 39 (11.996) \\
\hline Obese & $60(10.6 \% 6)$ & $30(12.6 \% 6)$ & $30(9.296)$ \\
\hline \multicolumn{4}{|l|}{ Ethnicity $(n, 96)$} \\
\hline White/Caucasian & $373(66696)$ & $160(68.196)$ & $213(65.606)$ \\
\hline African American & $15(27 \%)$ & $4(1.796)$ & $11(3.496)$ \\
\hline Asian & $57(10.296)$ & $25(10.6 \% 6)$ & $32(9.906)$ \\
\hline First Nations & $2(0.496)$ & $1(0.406)$ & $1(0396)$ \\
\hline East Indian & $5(0.996)$ & $1(0.406)$ & $4(1.296)$ \\
\hline Do not know/other & 108 (19.3\%) & $11(18.7 \%)$ & $64(19.696)$ \\
\hline \multicolumn{4}{|l|}{ Physical activity (min/day) } \\
\hline MVPA & 58.7 (19.3) & $67.1(19.3)$ & $527(17.0)^{*}$ \\
\hline Moderate & $41.8(12.1)$ & $47.6(11.7)$ & $37.8(10.7)^{*}$ \\
\hline Light PA & $304.8(45.0)$ & $310.1(43 \mathrm{~J})$ & $301.1(45.5)^{\cdots \cdots}$ \\
\hline Total SED (min/day) & $511.4(63.1)$ & $506.9(660)$ & $514.5(60.9)$ \\
\hline \multicolumn{4}{|c|}{ Self-reported screen time score (hour/day) } \\
\hline Total screen time & $28(1.8)$ & $2.9(1.6)$ & $2.7(1.9)$ \\
\hline TV & $1.5(1.3)$ & $1.5(1.1)$ & 1.6 (1.2) \\
\hline Video game/computer & $1.3(1.0)$ & $1.4(1.0)^{*}$ & $1.1(1.0)$ \\
\hline \multicolumn{4}{|l|}{ Household income $(n, \%)$} \\
\hline Less than $\$ 14,000$ & $16(30)$ & $5(2.2)$ & $11(3.5)$ \\
\hline$\$ 15,000-59,999$ & $89(16.4)$ & $33(14.4)$ & $56(17.7)$ \\
\hline$\$ 60,000-139,999$ & $231(424)$ & $100(43.7)$ & $131(39.4)$ \\
\hline$\$ 140,000$ and above & $209(384)$ & $91(39.7)$ & $118(373)$ \\
\hline \multicolumn{4}{|l|}{ Parental characteristics } \\
\hline \multicolumn{4}{|l|}{ Mother } \\
\hline \multicolumn{4}{|l|}{ Education } \\
\hline High school or less & $85(15.3)$ & $35(15.1)$ & $50(15.4)$ \\
\hline Greater than high school & $473(84.7)$ & $198(85.0)$ & $275(84.6)$ \\
\hline Self-reported BMI $\left(\mathrm{kg} / \mathrm{m}^{2}\right)$ & $24.9(5.2)$ & $25.3(5.6)$ & $24.6(4.9)$ \\
\hline Age (years) & $41.7(5.1)$ & $41.5(4.9)$ & $41.9(5.2)$ \\
\hline \multicolumn{4}{|l|}{ Father } \\
\hline \multicolumn{4}{|l|}{ Education } \\
\hline High school or less & $102(187)$ & $40(17.7)$ & $56(193)$ \\
\hline Greater than high school & $444(81.3)$ & $185(82.3)$ & $259(80.7)$ \\
\hline Self-reported BMI $\left(\mathrm{kg} / \mathrm{m}^{2}\right)$ & $26.8(4.4)$ & $26.7(4.4)$ & $26.8(4.4)$ \\
\hline Age (years) & $44.1(6.0)$ & $44.4(6.2)$ & $43.8(5.8)$ \\
\hline
\end{tabular}


Table 3 Univariate correlates of total sedentary time ${ }^{a}$

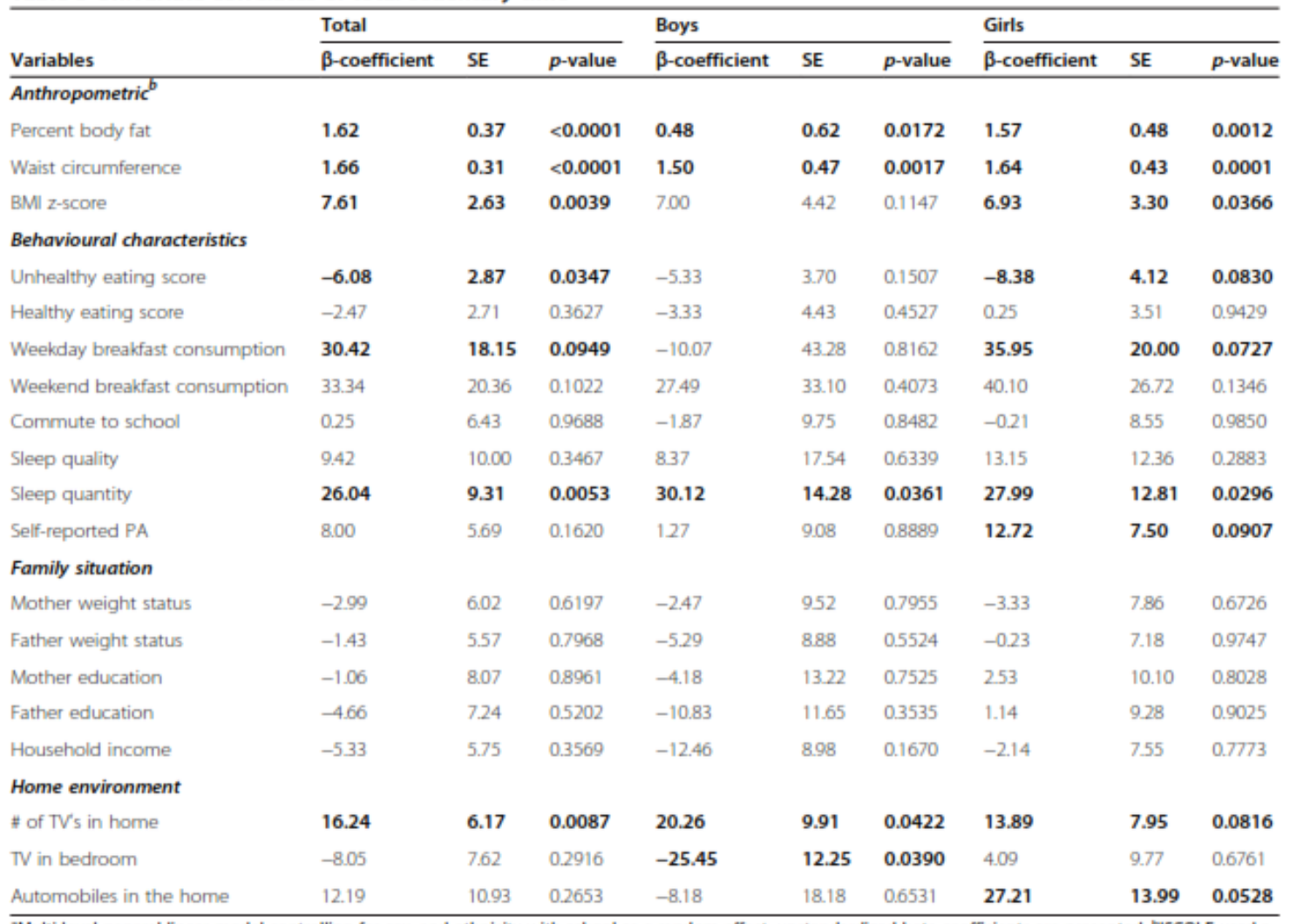

"Multi-level general linear model controlling for sex, and ethnicity with school as a random effect, unstandardized beta coefficients are presented. $15 \mathrm{SCOLE}$ used a variety of measures to assess adiposity, all of which were significant in univariate analyses. To build the final models, stepwise addition was used to determine which measure of adiposity provided the closest fit for the data. Akaike information criterion, Bayesian information criterion, and level of significance were used to determine model fit. Waist circumference alone provided the best fit.

PA: physical activity; SE: Standard Error; TV: television.

NOTE: bolded data indicates significance $(p<0.10)$.

healthy eating pattern score and weekend breakfast consumption

\section{Discussion}

This study aimed to identify correlates of accelerometer measured SED and self-reported ST in 10 year-old Canadian children. In the total sample, common correlates of SED and ST included waist circumference (positive association) and number of TVs in the home (positive association). In the total sample, increased SED was also associated with poor sleep quantity. We identified a greater number of correlates of ST than for SED. Specifically, correlates of ST in the total sample also included mother's weight status (negative association), father's education (positive association), unhealthy eating pattern score (positive association), healthy eating pattern score (negative association), and weekend breakfast consumption (negative association). Correlates identified here are similar to those identified in previous studies [11]. However, this is one of few studies to identify correlates of both SED and ST in the same population, and the first to examine this in a sample of Canadian children. This information can be used to help inform public health strategies and messages. Specifically, results from this work suggest that to reduce both SED and ST, public health messages (and interventions) focus on healthy weights, and reducing the number of TVs present in the house.

A positive association between waist circumference, and both SED and ST is consistent with previous work in boys and girls [27], and in both younger [27] and older [28] children. Although the cross-sectional nature of ISCOLE cannot provide information on causality, findings from a previous study show that fat mass is a significant predictor of sedentary time, but sedentary time is not a predictor of fat mass in children of the same age [29]. This is consistent with numerous other studies that have shown a 
Table 4 Univariate correlates of self-reported screen time ${ }^{a}$

\begin{tabular}{|c|c|c|c|c|c|c|c|c|c|}
\hline \multirow[b]{2}{*}{ Variables } & \multicolumn{3}{|l|}{ Total } & \multicolumn{3}{|l|}{ Boys } & \multicolumn{3}{|l|}{ Girls } \\
\hline & $\beta$-coefficient & SE & p-value & $\beta$-coefficient & SE & p-value & $\beta$-coefficient & SE & p-value \\
\hline \multicolumn{10}{|l|}{ Anthropometric } \\
\hline Percent body fat & 0.01 & 0.00 & 0.0118 & 0.01 & 0.01 & 0.0677 & 0.01 & 0.01 & 0.0329 \\
\hline Waist circumference & 0.01 & 0.00 & 0.0010 & 0.01 & 0.00 & 0.0325 & 0.01 & 0.01 & 0.0045 \\
\hline BMI z-score & 0.07 & 0.03 & 0.0085 & 0.07 & 0.04 & 0.0930 & 0.09 & 0.04 & 0.0167 \\
\hline \multicolumn{10}{|l|}{ Behavioural characteristics } \\
\hline Unhealthy eating score & 0.13 & 0.03 & $<0.0001$ & 0.08 & 0.03 & 0.0248 & 0.26 & 0.05 & $<0.0001$ \\
\hline Healthy eating score & -0.11 & 0.03 & $<0.0001$ & 0.07 & 0.03 & 0.0248 & 0.26 & 0.05 & $<0.0001$ \\
\hline Weekday breakfast consumption & 0.07 & 0.18 & 0.7118 & -0.21 & 0.41 & 0.6117 & -0.12 & 0.21 & 0.5883 \\
\hline Weekend breakfast consumption & -0.45 & 0.20 & 0.0250 & -0.09 & 0.27 & 0.7545 & -0.66 & 0.30 & 0.0257 \\
\hline Commute to school & -0.09 & 0.06 & 0.1634 & -0.02 & 0.09 & 0.8381 & -0.14 & 0.09 & -0.1384 \\
\hline Sleep quality & 0.09 & 0.10 & 0.3687 & 0.31 & 0.14 & 0.0309 & -0.05 & 0.13 & 0.6803 \\
\hline Sleep quantity & 0.15 & 0.09 & 0.1055 & 0.25 & 0.13 & 0.0507 & 0.08 & 0.14 & 0.5728 \\
\hline Self-report PA & 0.13 & 0.06 & 0.0213 & 0.17 & 0.08 & 0.0427 & 0.11 & 0.08 & 0.1880 \\
\hline \multicolumn{10}{|l|}{ Family situation } \\
\hline Mother weight status & 0.19 & 0.06 & 0.0011 & 0.10 & 0.08 & 0.2612 & 0.29 & 0.08 & 0.0005 \\
\hline Father weight status & 0.09 & 0.06 & 0.1001 & 0.11 & 0.08 & 0.1678 & 0.10 & 0.08 & 0.2157 \\
\hline Mother education & -0.15 & 0.08 & 0.0596 & -0.24 & 0.08 & 0.2731 & -0.19 & 0.11 & 0.0837 \\
\hline Father education & -0.35 & 0.07 & $<0.0001$ & -0.21 & 0.11 & 0.0459 & -0.47 & 0.10 & $<0.0001$ \\
\hline Household income & -0.19 & 0.06 & 0.0012 & -0.24 & 0.08 & 0.0038 & -0.20 & 0.08 & 0.0144 \\
\hline \multicolumn{10}{|l|}{ Home environment } \\
\hline \# of TV's in home & 0.15 & 0.06 & 0.0153 & 0.24 & 0.09 & 0.0090 & 0.11 & 0.09 & 0.2041 \\
\hline TV in bedroom & -0.32 & 0.07 & $<0.0001$ & -0.28 & 0.11 & 0.0093 & -0.39 & 0.10 & 0.0002 \\
\hline Automobiles in home & -0.00 & 0.12 & 0.9684 & 0.07 & 0.17 & 0.6630 & -0.08 & 0.15 & 0.5696 \\
\hline
\end{tabular}

significant relationship between higher weight status/adiposity and higher levels of sedentarism in children and in adults $[4,30]$. Recent work in adults has shown that individuals with high accelerometer measured SED, and individuals with high self-reported ST are more likely to have metabolic syndrome compared to those in the lower SED and ST groups [31]. We also saw that mother's weight status (being overweight or obese) was a predictor for higher ST in girls, but not boys ( $\mathrm{p}<0.0001$, data not shown).

Our work showed a significant relationship between unhealthy/healthy eating pattern scores and ST, but not with SED. This may be partly due to increased energy intake, primarily through energy dense snacking, while watching TV [32]. Previous work has shown that both boys and girls who report high levels of reading or homework consume significantly less percent of energy from fat that those who reported low levels of reading or homework [33]. Consistent with previous work
$[33,34]$, we showed that higher scores unhealthy eating pattern (i.e., a more unhealthy diet) and lower scores for healthy eating pattern (i.e., a more healthy diet) are correlates of ST for boys and girls. Previous research from Utter et al. showed that girls and boys who reported the highest screen time consumed 300-400 calories more per day than those who reported the lowest screen time [33]. Although we are unable to comment on the quantity (i.e., number of calories) of energy intake, the association of ST with consumption of energy-dense foods, such as soft drinks, fried food, and unhealthy snacks, is consistent with current results, as well as other work using the ISCOLE Canada dataset [35].

For SED, potential correlates (except weight status and healthy/unhealthy diet scores) were re-coded as dichotomous variables. The advantage of this approach was that all variables were on approximately the same scale, so that the strength of the association of each correlate 


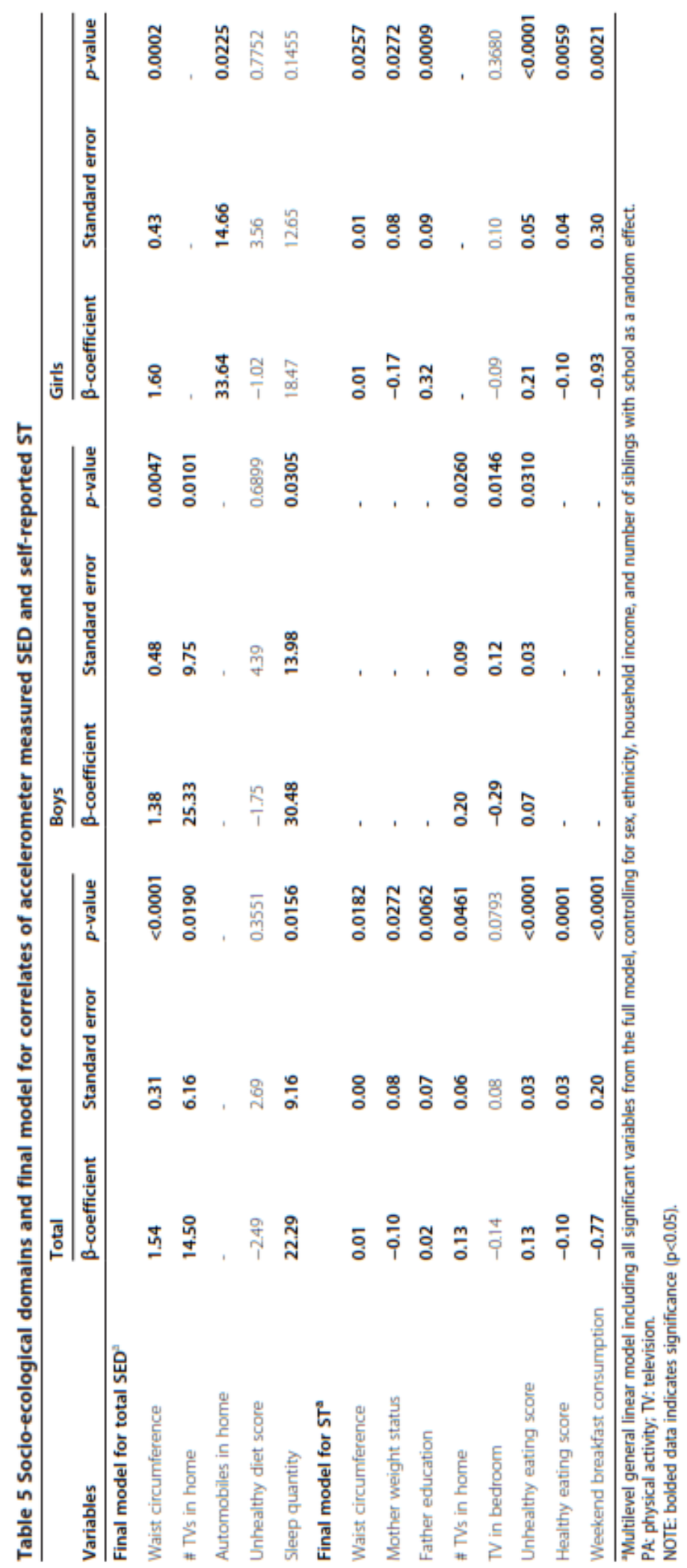


could be examined, and interpreted as a proportional increase in the outcome variable (e.g., a unit increase in the beta coefficient for a correlate of SED, represented an approximate increase of one minute per day of SED) (Table 5). This information can help inform public health interventions to reduce SED. Poor self-reported sleep quantity represented an approximate increase of 22.3 minutes of SED per day, and having more TVs in the home represented an approximate increase in 14.5 minutes of SED per day. Because ST was measured as a score, instead of hours per day, it could not be interpreted the same way, but strength of association can still examined. Not eating breakfast on the weekends and having two or more TVs in the house was associated with the largest increase in ST score.

Self-reported number of days being physically active for greater than 60 minutes was not a correlate of SED or ST. To understand the significance of this finding, we re-ran the models to include accelerometer-derived variables (intensities as well as average counts per minute) instead of the self-report physical activity (data not shown). When included, LPA, MVPA, and total accelerometer activity counts were all found to be significant correlates of SED, but not for ST. This was true when including the movement variables by themselves, or when including combinations of different intensity levels. There is debate as to whether to include more than one movement variable in the same model because although they may not be highly correlated (i.e., no issues with multicollinearity), they are also dependent on each other since they are derived from the same measurement device (i.e. proportional error). Including accelerometer-derived activity variables can also mask variance that could be attributed to other potential correlates.

Consistent with previous work, we found that selfreported ST explained only a small portion (approximately $33 \%$ ) of accelerometer measured SED [10]. ST did have an upper limit of 10 hours per day (5 hours of TV viewing and 5 hours of video/computer games) but few participants reported $>5$ hours of TV per day ( $3 \%$ and $9 \%$ on weekdays and weekend days, respectively) or $>5$ hours of computer/video games per day ( $1 \%$ and $6 \%$ on weekdays and weekend days, respectively). While accelerometers are able to provide accurate information on activity for the whole day, they are not able to detect the difference between some activities (e.g., standing still versus sitting still), and are not able to provide any context for the behaviour (e.g., reading versus watching TV) [36]. This suggests that there is a large proportion of daily SED that is unaccounted for by measuring ST alone. Future work should aim to understand correlates, and related health effects (both positive and negative) of other, non-screen based sedentary behaviours (e.g., reading a book, passive transportation).
Future work should also aim to understand how technology influences the use of electronic devices in daily life. This is important for health care providers when planning public health strategies to reduce SED and ST, and for sedentary behaviour researchers when defining SED and ST. For example, with the advent of smart phones, and tablet computers, sedentary multi-tasking is common; a child is able to watch TV, play on the computer, and use a smart phone at the same time. Further, a device can be multi-purpose (e.g., can be used as TV, video game, book, musical instrument, computer, etc.). We know very little about how this affects healthy growth and development. Future work needs to better understand possible associations (negative or positive) with both screen- and nonscreen based behaviours to help inform public health messages.

This paper has both strengths and limitations. Accelerometers have been shown to be a valid tool to measure activity of all levels of intensity. However, it is wellunderstood that a hip-placed monitor is less effective in distinguishing sedentary postures, like lying or sitting, from other very light intensity activities performed while standing; further, accelerometers cannot accurately capture upper body movements, cycling, or activities when the monitor is removed. For ISCOLE, algorithms were used to distinguish sleep from SED. The algorithms have been validated and published [37], and accelerometer cut points for SED have high sensitivity [19], but there is always the possibility of misclassification. Many potential correlates of SED and ST were based on child- or parent-report, which is subject to either over- or under-reporting. It is also possible that these correlates are unique to Canadian children in the current study, and caution should be used when generalizing these results to other populations. Further, it would have been beneficial to include additional potential correlates related to the family environment (e.g., parenting rules and restrictions relating to ST, parental ST habits) [38]. Finally, causality of correlates identified here cannot be determined from cross-sectional data and future studies using a longitudinal design will be needed to address this issue.

\section{Conclusion}

This study aimed to identify correlates of objectively measured total SED and self-reported ST in Canadian children. For both SED and ST, some of the correlates we identified are easily modifiable (e.g., reducing the number of TVs present in the home), while others may require more intense behavioural interventions (e.g, reducing waist circumference). Overall, we were able to identify a wider variety of correlates of ST than of SED. Future work should examine the relationship between a range of sedentary behaviours, including non-screen based pursuits, across a more 
geographically and culturally distinct population to inform public health strategies and messages.

\section{Abbreviations}

BMl: Body mass indexc LPA: Light-intensity physical activity; MVPA: Moderate- to vigorous-intensity physical activity, PA: Physical activity; SED: Accelerometer measured total sedentary time; ST: Self-report screen time; TV: Television.

\section{Competing interests}

The authors declare they have no competing interests; ISCOLE was funded by the Coca-Cola Company. The funder had no role in study design, data collection and analysis, decision to publish or preparation of this manuscript.

\section{Authors' contributions}

AGL, MST, STB, and JPC conceived of, and designed the study. AGL and STB planned and preformed the analysis. All authors participated in the interpretation of analysis. AGL drafted the manuscript. All authors critically revised the manuscript and approved of it in its final form.

\section{Acknowledgements}

We thank Priscilla Belanger, Claire Francis, Jessica McNeil, Hadiza Amedu-Ode and Nina Azoug-Boneault for their role in data collection for the Canadian site of ISCOLE, and the Coordinating Center of ISCOLE in Baton Rouge, Louisiana, specifically Drs Peter Katzmarzyk and Timothy Church. We also thank the study participants along with their parents, teachers and school principals for their inwolvernent in the study.

\section{Author details}

'Healthy Active Living and Obesity Research Group, Children's Hospital of Eastern Ontario Research Institute, 401 Smyth Road, Ottawa, Ontario K1H $8 \mathrm{LI}$, Canada. 2Population Health, Faculty of Graduate and Postdoctoral Studies, University of Ottawa, Ottawa, Ontario, Canada. "Pennington Biomedical Research Center, Baton Rouge, Louisiana, US. "Department of Pediatrics, Faculty of Medicine, University of Ottawa, Ottawa, Ontario, Canada. "School of Human Kinetics, Faculty of Health Sciences, University of Ottawa, Ottawa, Ontario, Canada

\section{Received: 8 September 2014 Accepted: 2 March 2015}

\section{Published online: 18 March 2015}

\section{References}

1. Sedentary Behaviour Research Networ. Letter to the Editor. Standardized use of the terms "sedentary" and "sedentary behaviours.". Appl Physiol Nutr Metab. 2012:37:540-2

2 Tremblay MS, Colley AC, Saunders TJ, Healy GN, Owen N. Physiological and health implications of a sedentary lifestyle. Appl Physiol Nutr Metab. 2010:35:725-40.

3. Tremblay MS, LeBlanc AG, Janssen L, Kho ME, Hicks A Murumets K, et al Canadian Sedentary Behaviour Guidelines for Children and Youth. Appl Physiol Nutr Metab. 2011;3659-64.

4 Tremblay MS, LePlanc AG, Kho ME, Saunders TI L arouche R, Colley RC et al Systematic review of sedentary behaviour and health indicators in school-aged children and youth. Int J Behav Nutr Phys Act. 2011,898

5. Wilmot EG, Edwardson CL, Achana FA, Davies MJ, Gorely T, Gray $\sqcup$ et al. sedentary time in aduits and the association with diabetes, cardiovascular disease and death systematic review and meta-analysis. Diabetol Clin Exp Diabetes Metab. 2012

6. Healy GN, Dunstan DW, Salmon J, Cerin E, Shaw JE, Zimmet PZ, et al. Breaks in Sedentary Time Beneficial associations with metabolic risk. Diabetes Care. 2008:31.661-6.

7. Healy GN, Matthews CE, Durstan DW, Winkler EAH, Owen N. Sedentary time and cardio-metabolic biomarkers in US adults: NHANES 2003-06. Eur Heart 1. 2011:32590-7.

8. Saunders TJ, Chaput J.P, Goldfield GS, Colley RC, Kenny GP, Doucet E, et al. Prolonged sitting and markers of cardiometabolic disease risk in children and youth: a randomized crossover study. Metabolism. 2013:62:1423-8,

9. Colley RC, Garriguet D, Janssen I, Wong SL, Saunders TJ, Carson V, et al. The association between accelerometer-measured patterns of sedentary time and health risk in children and youth: results from the Canadian Health Measures Survey. BNC Public Health. 2013:13-200.
10 Biddle S.H. Gorely T, Marshall SI, Is television viewing a suitable marker of sedentary behavior in young people? Ann Behav Med. 2009:38:147-53.

11. Temmel CSD, Phodes R Correlates of Sedentary Behaviour in Children and Adolescents Aged 7-18: A Systematic Review. Health Fit J Can. 2013,6:119-99.

12. Brodarsen NH. Steptoe A Willamson S, Wardle J. Sociodemographic, developmental, environmental, and psychological correlates of physical activity and sedentary behavior at age 11 to 12. Ann Behav Med. 2005;29:2-11.

13. Van Der Horst K. Paw MJ CA. Twisk JWR. Van Mechelen W. A brief review on correlates of physical activity and sedentariness in youth. Med Sci Sports Exerc. 2007;39:1241-50.

14. van Sluijs EMF, Page A. Ommundsen Y, Griffin SJ. Behavioural and social correlates of sedentary time in young people. Br J Sports Med. 2010:44:747-55.

15. Owen N, Sugiyama T, Eakin EE, Gardiner PA, Tremblay MS, Sallis JF. Adults' sedentary behavior: determinants and interventions. Am J Prev Med. 2011:41:189-96

16. Katzmarzkk PT, Barreia TV, Broves ST, Champagne CM, Chaput J.P. Fogeholm M, et al The Intemational Study of Childhood Obesity, Lfestyle and the Environment (SCOCF), design and methods. BMC Public Heath. 2013;13900.

17. Trost SG, Loprinzi PD, Moore R. Pfeiffer KA. Comparison of accelerometer cut points for predicting activity intensity in youth. Med Sci Sports Exerc. 201 1:43:1360-8

18. Colley RC, Connor Gorber S, Tremblay MS. Quality control and data reduction procedures for accelerometry-derived measures of physical activity. Health Rep. 2010:21:63-9.

19. Evenson KR, Catellier DJ, Gill K, Ondrak KS, McMurray RG. Calibration of two objective measures of physical activity for children. J Sports Sci. 2008:26:1557-65.

20. US. Centers for Disease Control and Prevention. Youth Pisk Behavior Surveillance System (YRBSS). 2012. Available at: httpt//www.codcgow/ HealthyYouth/yrbs.

21. Hardy LL, Hills AP. Timperio A, Cliff D, Lubans D, Morgan PJ, et al. A hitchhiker's guide to assessing sedentary behaviour among young people Deciding what method to use. J Sci Med Sport. 2013;16:28-35.

22. Barreira TV, Staiano AE, Katzmarzyk PT. Validity assessment of a portable bioimpedance scale to estimate body fat percentage in White and AfricanAmerican children and adolescents. Pediatr Obes. 20138:e29-32.

23. Cenre for Disease Control and Prevention. Assessing Your Weight: Children's BMII Tool for School. (Healthy Weight: It's Not a Diet. It's a Lifestylel. 2014.

24. Currie C. Nic Gabhainn S, Godeau E. Roberts C. Smith R. Currie D, et al. Inequalities in Children's Health: HBSC International Report from the 2005/ 2006 Survey. Geneva. World Health Organization; 2008.

25. Kenward MG, Roger IH Small Sample Inference for Fixed Effects from Restricted Maximum Likelihood. Biometrics. 199753.983.

26. Craney TA Surles JG. Model-dependent variance inflation factor cutoff values. Qual Eng. 2002:14:391.

27. Byun W. Dowda M. Pate RR. Correlates of obiectively measured sedentary behavior in US preschool children. Pediatrics. 2011;128:937-45.

28. Gorely T. Couch kids: Correlates of television viewing among youth. Int 1 Behav Med. 2004:11:152-63.

29 Hiorth MF. Chaput J.P, Fitz C, Dalskov S-M, Andersen R, Astrup A et al. Fatness predicts decreased physical activity and increased sedentary time, but not vice versa: support from a longitudinal study in 8- to 11 -year-old children. Int J Obes. 2014:38:959-65.

30. Saunders TJ, Larouche R, Colley RC, Tremblay MS. Acute sedentary behaviour and markers of cardiometabolic risk a systematic review of intervention studies. J Nutr Metab. 2012;2012:712435.

31. Saleh D, Janssen I. Interrelationships among sedentary time, sleep duration, and the metabolic syndrome in adults. BMC Public Health. 2014,14,660

32. Thivel D, Tremblay MS, Chaput J.P. Modern sedentary behaviors favor energy consumption in chidren and adolescents. Curr Obes Pep. 2013.250-7.

33. Utter J, Neumark-Sztainer D, Jeffery R. Story M. Couch potatoes or french fries: are sedentary behaviors associated with body mass index, physical activity, and dietary behaviors among adolescents? J Am Diet Assoc 2003:103:1298-305.

34. Pearson N. Biddle 5.H. Sedentary behavior and dietary intake in children, adolescents, and adults: a systematic review. Am J Prev Med. 2011,41:178-88

35. Borghese MM, Tremblay MS, Leduc $G$, Boyer $C$, Bélanger $P$, LeBlanc AG et al. Independent and combined associations of total sedentary time and television viewing time with food intake patterns of 9- to 11-year-old Canadian children. Appl Physiol Nutr Metab. 2014:1-7. 
36. Atkin AJ, Gorely T, Clemes SA, Yates T, Edwardson C, Brage S, et al. Methods of measurement in epiderniology: sedentary behaviour. Int J Epidemiol. 2012:41:1460-71

37. Tudor-Locke C, Barreira TV, Schuna JM, Mire EF, Katzmarzyk PT. Fulky autornated waist-worn accelerometer algorithm for detecting children's sleep-period time separate from 24-h physical activity or sedentary behaviors. Appl Physiol Nutr Metab. 2013:3953-7.

38. Granich J, Rosenberg M, Knuiman M, Timperio A. Understanding children's sedentary behaviour. a qualitative study of the family home environment. Health Educ Res. 2010:25:199-210.

Submit your next manuscript to BioMed Central and take full advantage of:

- Convenient online submission

- Thorough peer review

- No space constraints or color figure charges

- Immediate publication on acceptance

- Inclusion in PubMed, CAS, Scopus and Google Scholar

- Research which is freely available for redistribution 
4.3 Correlates of total sedentary time and screen time in 9-11 year-old children around the world: The International Study of Childhood Obesity, Lifestyle and the

\section{Environment}

This manuscript has been written and formatted to adhere to the specifications for submission to PLOS ONE. This article has been published and can be found here: http://journals.plos.org/plosone/article?id=10.1371/journal.pone.0129622 


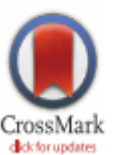

\section{GoPENACCESS}

Citation: LeBlanc AG, Katzmarzyk PT, Barreira TV, Broyles ST, Chaput J-P, Church TS, et al. (2015) Correlates of Total Sedentary Time and Screen Time in 9-11 Year-Old Children around the World: The International Study of Chilchood Obesity, L Lestyle and the Environment. PLOS ONE 10(6): e0129622. doi:10.1371.journal.pone.0129622

Academic Editor: Qinghua Sun, The Ohio State University, UNITED STATES

Received: February 19, 2015

Accepted: May 10, 2015

Published: June 11, 2015

Copyright: $\odot 2015$ LeBlanc et al. This is an open access article distributed under the terms of the Creafive Commons Attribution License, which permits unrestricted use, distribution, and reproduction in any medium, provided the original author and source are credited.

Data Availability Statement: Data are avalable upon request from Peter T. Katzmarzyk ( Peter. Katzmarzyk@pbrc.edu) through a data use agreement with Pennington Biomedical Research Center.

Funding: ISCOLE was funded by The Coca-Cola Company. The funder had no role in the design and conduct of the study; collection, management, analysis and interpretation of the data; and preparation, review or approval of the manuscript.
RESEARCH ARTICLE

\section{Correlates of Total Sedentary Time and Screen Time in 9-11 Year-Old Children around the World: The International Study of Childhood Obesity, Lifestyle and the} Environment

\author{
Allana G. LeBlanc ${ }^{1,2 *}$, Peter T. Katzmarzyk ${ }^{3}$, Tiago V. Barreira ${ }^{3,4}$, Stephanie T. Broyles ${ }^{3}$, \\ Jean-Philippe Chaput ${ }^{1,2}$, Timothy S. Church ${ }^{3}$, Mikael Fogelholm ${ }^{5}$, Deirdre M. Harrington ${ }^{\circ}$, \\ Gang Hu${ }^{3}$, Rebecca Kuriyan ${ }^{7}$, Anura Kurpad ${ }^{7}$, Estelle V. Lambert ${ }^{8}$, Carol Maher ${ }^{9}$, \\ José Maia ${ }^{10}$, Victor Matsudo ${ }^{11}$, Timothy Olds ${ }^{9}$, Vincent Onywera ${ }^{12}$, Olga L. Sarmiento ${ }^{13}$, \\ Martyn Standage ${ }^{14}$, Catrine Tudor-Locke ${ }^{3}$, Pel Zhao ${ }^{15}$, Mark S. Tremblay ${ }^{1}$, ISCOLE \\ Research Group"
}

1 Children's Hospital of Eastem Ontario Research Institute, Ottawa, Canada, 2 University of Ottawa, Ottawa, Canada, 3 Pennington Biomedical Research Center, Baton Rouge, United States of America, 4 University of Syracuse, Syracuse, United States of America, 5 University of Helsinki, Helsinki, Finland, 6 University of Leicester, Leicester, United Kingdom, 7 St. Johns Research Institute, Bangalore, India, 8 University of Cape Town, Cape Town, South Africa, 9 School of Health Sciences / Sansom Institute, University of South Australia, Adelaide, Australia, 10 Faculdade de Desporto, University of Porto, Porto, Portugal, 11 Centro de Estudos do Laboratório de Aptidăo Fisica de São Caetano do Sul (CELAFISCS), Sao Paulo, Brazil, 12 Kenyatta University, Nairobi, Kenya, 13 School of Medicine, Universidad de los Andes, Bogota, Colombia, Colombia, 14 University of Bath, Bath, United Kingdom, 15 Tianjin Women's and Children's Health Center, Tianjin, China

ๆ Members of the ISCOLE Research Group are presented in the Acknowledgments.

* alleblanc Qcheo on ca

\section{Abstract}

\section{Purpose}

Previously, studies examining correlates of sedentary behavior have been limited by small sample size, restricted geographic area, and little socio-cultural variability. Further, few studies have examined correlates of total sedentary time (SED) and screen time (ST) in the same population. This study aimed to investigate correlates of SED and ST in children around the world.

\section{Methods}

The sample included 5,844 children $(45.6 \%$ boys, mean age $=10.4$ years) from study sites in Australia, Brazil, Canada, China, Colombia, Finland, India, Kenya, Portugal, South Africa, the United Kingdom, and the United States. Child- and parent-reported behavioral, household, and neighborhood characteristics and directly measured anthropometric and accelerometer data were obtained. Twenty-one potential correlates of SED and ST were examined using multilevel models, adjusting for sex, age, and highest parental education, 
Competing Interests: ISCOLE was funded by The Coca-Cola Company. There are no patents, products in development or marketed products to declare. This does not alter the authors' adherence to al the PLOS ONE policies on sharing data and materials. with school and study site as random effects. Variables that were moderately associated with SED and/or ST in univariate analyses $(p<0.10)$ were included in the final models. Variables that remained significant in the final models $(p<0.05)$ were considered correlates of SED and/or ST.

\section{Results}

Children averaged 8.6 hours of daily SED, and $54.2 \%$ of children failed to meet ST guidelines. In all study sites, boys reported higher ST, were less likely to meet ST guidelines, and had higher BMI Z-scores than girls. In 9 of 12 sites, girls engaged in significantly more SED than boys. Common correlates of higher SED and ST included poor weight status, not meeting physical activity guidelines, and having a TV or a computer in the bedroom.

\section{Conclusions}

In this global sample many common correlates of SED and ST were identified, some of which are easily modifiable (e.g., removing TV from the bedroom), and others that may require more intense behavioral interventions (e.g., increasing physical activity). Future work should incorporate these findings into the development of culturally meaningful public health messages.

\section{Introduction}

Physical inactivity and sedentary behavior have been independently associated with a wide range of negative health indicators including obesity, poor cardio-metabolic health, and poor psychosocial health [1-4]. Sedentary behavior is characterized by waking behaviors that require little energy expenditure and that occur in a sitting or reclined position [5]. Total sedentary time (SED) can be further classified by a variety of specific sedentary behaviors such as reading, playing quietly, watching television (TV), using the computer, or playing video games. Previous work has relied largely upon self-reported sedentary pursuits, and focused largely on screen time (ST), which usually entails some combination of watching TV, playing video games, and/or using the computer [4]. Although ST is often used as a proxy measure for SED, ST accounts for only about a third of SED [6], with the rest of the SED being spent in a variety of other pursuits such as reading a book, passive transport, or eating. With the widespread use of accelerometers, researchers are able to capture objectively measured SED throughout the day [7]. This is important since increased ST is consistently associated with poor health in children and youth, but the relationship between increased SED and increased disease risk is less clear $[8,9]$.

In developed countries, those in lower socio-economic levels and those belonging to ethnic minorities are more likely to be sedentary $[10,11]$. In under-developed, or developing countries, the trend is reversed, with those with the highest socio-economic status being most sedentary [12]. Previous work has also shown that early adolescence is a common time for levels of moderate- to vigorous-intensity physical activity to decline, and for SED (including ST) to increase $[10,13]$. Further, differences in levels of physical activity and SED due to socio-cultural factors are largely established during this developmental period [10]. Therefore, pre-adolescence may be a particularly important time to prevent the development of poor behavior 
habits, including decreased participation in physical activity of all intensities, and increased attraction to screen based SED.

A review examining the correlates of TV viewing in youth suggested that those who watched the most TV were pre-adolescents (i.e., aged 9-13 years), from families of lower socio-economic status, from single parent households, and those belonging to ethnic minorities (with African American children watching the most TV) [14]. However, this review was largely informed by North American studies ( $72 \%$ of included studies), and focused exclusively on TV viewing. The authors identify these limitations and go on to recommend that future studies use an ecological framework to understand a broad range of intrapersonal, interpersonal, and environmental correlates of sedentarism. Since this review, many studies have examined correlates of sedentarism, including several that have used direct (i.e., accelerometer) measurement to assess SED; however, they have been limited by small sample sizes, restricted geographic area, and/or little socio-cultural variability [15]. Temmel and Rhodes [15] identified 64 studies that examined the correlates of SED in young people. Although the review included studies from countries all over the world, very few studies included multi-national populations using the same methodology. In fact, the most geographically diverse study sample came from the Health $\mathrm{Be}$ haviors in School-aged Children survey (HBSC), which included only self-reported data from developed countries.

The International Study of Childhood Obesity, Lifestyle and the Environment (ISCOLE) represents the most current, directly-measured, harmonized dataset focused on lifestyle and obesity among children from all regions of the world. The aim of the present analysis was to investigate the correlates of SED and ST in a large multi-national sample of 10 year-old children from diverse cultural and socioeconomic backgrounds.

\section{Methods \\ Data Source}

ISCOLE is a multi-site, cross-sectional study conducted in 9-11 year-old children from sites in 12 different countries (Australia, Brazil, Canada, China, Colombia, Finland, India, Kenya, Portugal, South Africa, United Kingdom, and United States) [16]. The primary aim of ISCOLE was to determine the relationships between lifestyle behaviors and obesity in a multinational study of 9-11 year-old children. Further, ISCOLE aimed to investigate the influence of higher-order characteristics such as behavioral settings, and the physical, social and policy environments, on the observed relationships within and between countries [16]. To ensure ISCOLE represented a variety of backgrounds and circumstances, study sites were chosen from diverse geographic regions around the world (i.e., Europe, Africa, the Americas, SouthEast Asia, and the Western Pacific) and across different levels of socio-economic indicators (i.e., World Bank income classification, Human Development Index, and the Gini Index). Additional details on study design, participating countries, and full methodology have been published elsewhere [16].

Data collection for ISCOLE occurred from October 2011 until December 2013 with a goal of recruiting at least 500 participants, aged 9-11 years, from each study site. Data collection strategies varied slightly by ISCOLE site; details on site-specific recruitment strategies can be found in the ISCOLE methods paper [16]. The ISCOLE coordinating center, located at the Pennington Biomedical Research Center in Baton Rouge, Louisiana, was responsible for overall administration of the study. This project was approved by the relevant research ethics boards at Pennington Biomedical Research Center, at each ISCOLE study site, and at the respective school boards. Written informed parental consent and child assent were obtained for all participants. 


\section{Dependent Variables}

Accelerometer measured sedentary time. The ActiGraph GT3X+triaxial accelerometer (ActiGraph LLC, Pensacola, FL, USA) was used to objectively measure SED. Participants were asked to wear the accelerometer, attached to an elastic belt around the waist at the right midaxillary line, for 7 consecutive days, 24 hours/day, removing only for water activities (e.g., bathing, swimming). Children received instruction during the initial in-school assessment on how to wear the accelerometer. To increase compliance a variety of measures were used across different countries including in-person compliance checks, and phone calls to the participants' parents to ensure the child was wearing the accelerometer correctly.

Data reduction strategies limited the analytical dataset to participants who provided at least four days of valid measurements (including at least one weekend day), with at least 10 hours/ day of waking wear time $[17,18]$. Data were collected at a sampling rate of $80 \mathrm{~Hz}$, downloaded in 1-second epochs, and aggregated to 15-second epochs for analysis [19]. To determine SED, total sleep period time and non-wear time were identified using validated procedures [20]. For the current analysis, SED was defined as all epochs showing $\leq 25$ counts/ 15 seconds, consistent with widely used cutoffs from Evenson et al. [19].

Self-reported screen time. Child-reported screen time was determined from a Diet and Lifestyle Questionnaire, with questions taken from the U.S. Youth Risk Behaviour Surveillance System $[16,21]$. Children were asked how many hours they typically watched TV, and how many hours they played video games and/or used the computer during their discretionary time, per week day, and per weekend day. Responses were: $0=\mathrm{I}$ did not watch TV, $1=\leq 1$ hour of TV, $2=2$ hours, $3=3$ hours, $4=4$ hours, $5=\geq 5$ hours of TV. A weighted mean score of hours of daily ST was calculated as follows: [(hours of TV on weekdays $x 5$ ) + (hours of TV on weekend days $\times 2$ ) + (hours of video games and computers on weekdays $x 5$ ) + (hours of video games and computers on weekend days $\times 2$ )]/7. For analysis, this is presented as a ST score, rather than total hours of ST since after 5 hours/day, we could not ascertain the participant's actual amount of ST. Self-reported methods for quantifying ST have acceptable reliability and validity in children [7]. After testing for normality, ST was log-transformed for analysis and analyzed as a continuous variable. ST was also presented as those who did not meet ST guidelines of $\leq 2$ hours/day [22].

\section{Potential Correlates}

Twenty-one potential correlates of SED and/or ST were included in the analysis. Correlates included directly measured, child-reported, and parent-reported variables and were chosen based on the previous literature and the plausibility of their relationship with SED and/or ST $[14,15]$. See Table 1 for details on response categories and additional measurement details.

Anthropometric variables. Anthropometric data (i.e., height, weight, waist circumference, percent body fat) were directly measured by trained ISCOLE researchers during an inschool visit according to standardized procedures and measurement tools [16]. Height (to the nearest $0.1 \mathrm{~cm}$ ) was measured using a Seca 213 portable stadiometer (Hamburg, Germany). Weight (to the nearest $0.1 \mathrm{~kg}$ ) and body fat percentage (to the nearest $0.1 \%$ ) were measured using a portable Tanita SC-240 Body Composition Analyzer (Arlington Heights, IL, USA). The Tanita SC-240 has shown acceptable accuracy for estimating body fat percentage when compared with dual-energy X-ray absorptiometry, supporting its use in field studies [23]. Body mass index (BMI) was calculated $(\mathrm{kg} / \mathrm{m} 2)$, and BMI z-score, based on age and sex, was determined using the World Health Organization (WHO) cut-offs for all participants [24]. Waist circumference (to the nearest $0.1 \mathrm{~cm}$ ) was measured using a non-elastic anthropometric tape after a normal exhalation, at the mid-point between the top of the iliac crest and the bottom of 


\section{PLOS | ONE}

Table 1. Potential correlates of objectively measured sedentary time (SED) and self-reported screen time (ST).

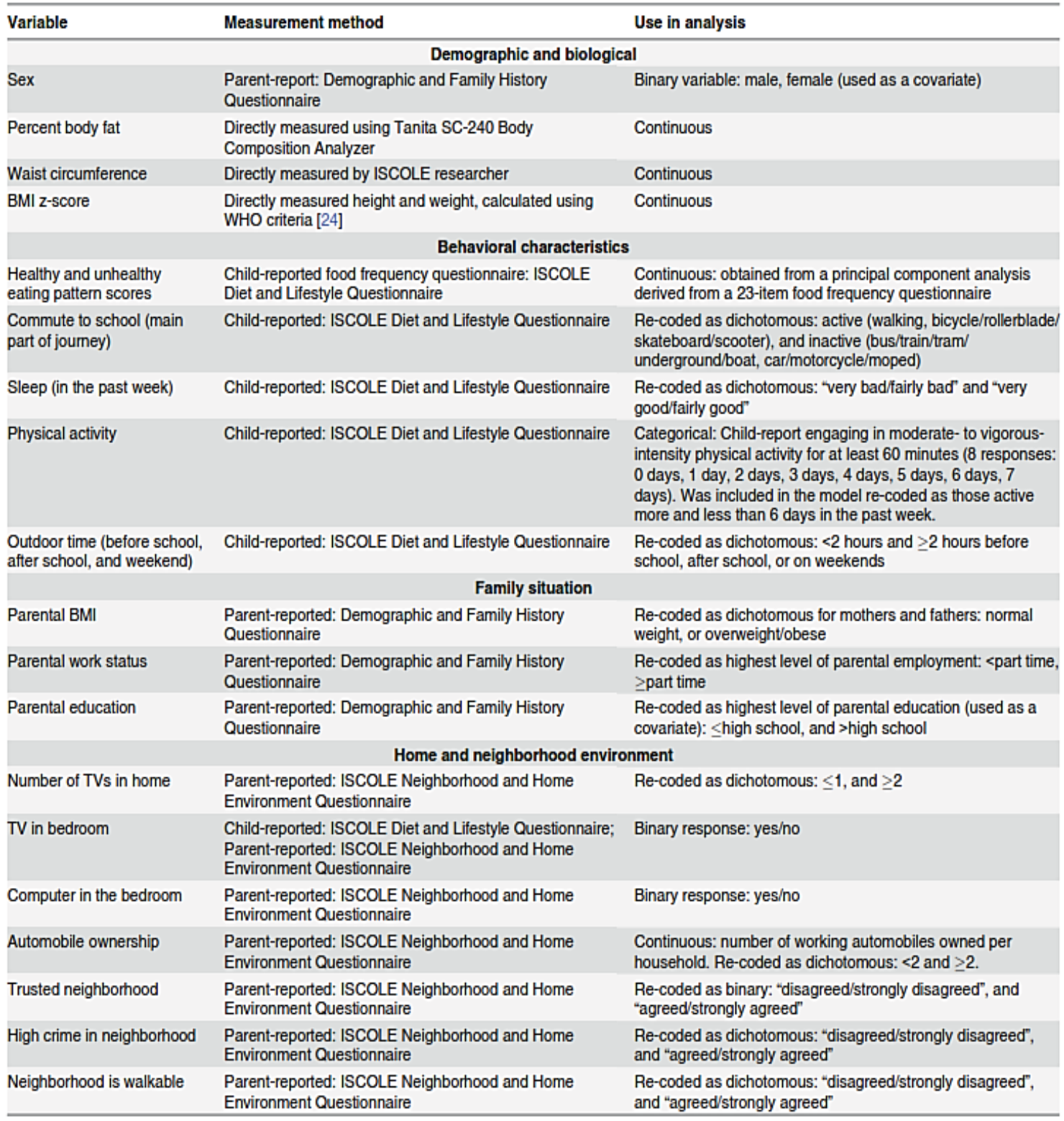

BMI: body mass index; ISCOLE: International Study of Childhood Obesity, Lifestyle and the Environment; TV: television; WHO: World Health Organization doi:10.1371.journal.pone.0129622.t001

the last floating rib, directly against the skin (except in Australia where measures were taken over light clothing).

Child-reported behavioral characteristics. Participants completed the ISCOLE Diet and Lifestyle Questionnaire, containing questions asked to the child related to dietary intake, 
physical activity, ST, and sleep [16]. The questionnaires were generally completed during the ISCOLE school visit, at the same time that anthropometric measurements were obtained and that the accelerometers were distributed. Children completed a Food Frequency Questionnaire (FFQ) adapted from the HBSC study [25], which asked how often they consumed 23 food items in a usual week. To identify existing dietary patterns among the study population, principal components analyses were carried out using the FFQ food groups as input variables (excluding fruit juices due to low validity) (unpublished analysis). Eigenvalues and a scree plot analysis were used as the criteria for deciding the number of factors extracted. The two criteria lead to similar conclusions and two factors were eventually chosen for each analysis. The factors were then rotated with an orthogonal varimax transformation to force non-correlation of the factors and to enhance the interpretation. Two factors were included in this manuscript as exposure variables: "unhealthy eating pattern score" (e.g., hamburgers, soft drinks, fried food; higher score means worse eating pattern) and "healthy eating pattern score" (e.g., vegetables and fruits; higher score means better eating pattern). Children were asked how they got to school most days (e.g., walking, car), how much time they spent outside (before school, after school, and weekend), and sleep quality and quantity. Finally, to assess if children met current physical activity guidelines, children were asked how many days they engaged in at least 60 minutes of moderate- to vigorous-intensity physical activity [26].

Parent-reported home and neighborhood environment. A Demographic and Family History Questionnaire, and a Neighborhood and Home Environment Questionnaire contained questions for parents related to their home and neighborhood environment [16]. The parent filling out the questionnaire was asked information specific for the "mother" and the "father". In particular, they were asked to report the highest level of education (ranging from "less than high school" to "post graduate degree"), employment (ranging from "not at all" to "full time"), height, and weight for themselves and their co-parent. The parent-report questionnaires were sent home with the child at the same time as the parent-consent forms and collected during the in-school visit. Parents were contacted if there was missing information, or if the responses needed to be clarified.

\section{Statistical Analysis}

Statistical analyses were conducted using SAS 9.4 (SAS Institute Incorporated, North Carolina, USA). Descriptive information (means, standard deviation, frequencies as appropriate) was calculated for demographic and anthropometric characteristics of all participants and their parents. Unpaired t-tests and chi-square tests were used to test differences between boys and girls. Multilevel general linear models (PROC MIXED), including school and ISCOLE study site as random effects, were used to determine correlates of SED and ST. Potential correlates (see Table 1 for details) were first included in univariate models; variables that were at least marginally significant $(\mathrm{p}<0.10)$, were subsequently included in domain-specific models similar to those outlined in the social ecological model proposed by Owen et al. for SED [27] (i.e., biological characteristics, behavioral characteristics, parental characteristics, and home and neighborhood environment). Variables that showed at least marginally significant associations $(\mathrm{p}<0.10)$ with SED or ST in the domain-specific models were included in the final model (results of domain-specific models not shown). Variables that remained significant $(\mathrm{p}<0.05)$ in the final model were considered correlates of SED and/or ST. To show significant correlates across sites, we ran the final models for SED and ST in each site country separately (keeping schools as a random effect). To show differences in levels of SED and ST between boys and girls in each site, we ran sex by site interactions and computed the least square means (LSMEANS) of fixed effects. 


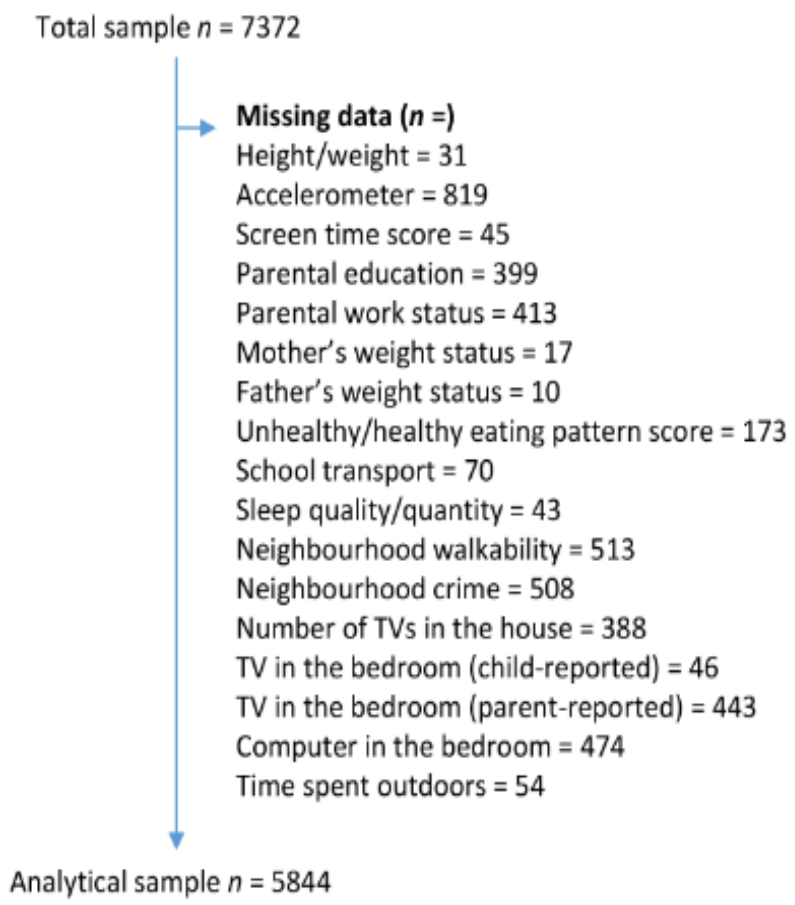

Fig 1. Participants with missing data. A total of 1528 participants were excluded due to missing data, some participants had missing data for more than one variable.

doi:10.1371.journal.pone.0129622.g001

Child's sex, age, and their parent's highest level of education were included as covariates for all models. These covariates were selected based on the plausibility of their potential confounding effect and because of their known associations with SED and/or ST [15]. The KenwardRoger approximation (DDFM $=\mathrm{KR}$ ) was used to calculate degrees of freedom [28]. Multicollinearity was tested using tolerance and variance inflation factors, and results indicated no issues with multicollinearity [29]. Analyses were conducted and presented for the total sample, and separately for boys and girls.

\section{Results}

Complete data for the outcomes of interest and all investigated correlates were available for 9-11 year-old children from Australia $(n=454)$, Brazil $(n=427)$, Canada $(n=502)$, China $(n=487)$, Colombia $(n=836)$, Finland $(n=542)$, India $(n=540)$, Kenya $(n=464)$, Portugal ( $n=547)$, South Africa $(n=306)$, United Kingdom $(n=407)$, and the United States ( $\mathrm{n}=422$ ). Missing data were found for 1528 participants (Fig 1); due to the large number of participants with missing data, sensitivity analysis was performed (Data not shown). Participants excluded from the present analysis engaged in similar levels of SED, but had higher BMI z-scores, and lower ST scores than those included in the analysis; more boys were also excluded from the analysis due to missing data. As per the ISCOLE study design, included countries varied considerably in their population size and level of wealth and human development. Across the total sample, the largest fraction of the total variance in both SED and ST was explained by individual-level factors ( $78.0 \%$ and $83.7 \%$, respectively), followed by sites 
Table 2. Descriptive characteristics of all participants $(n=5,844)$.

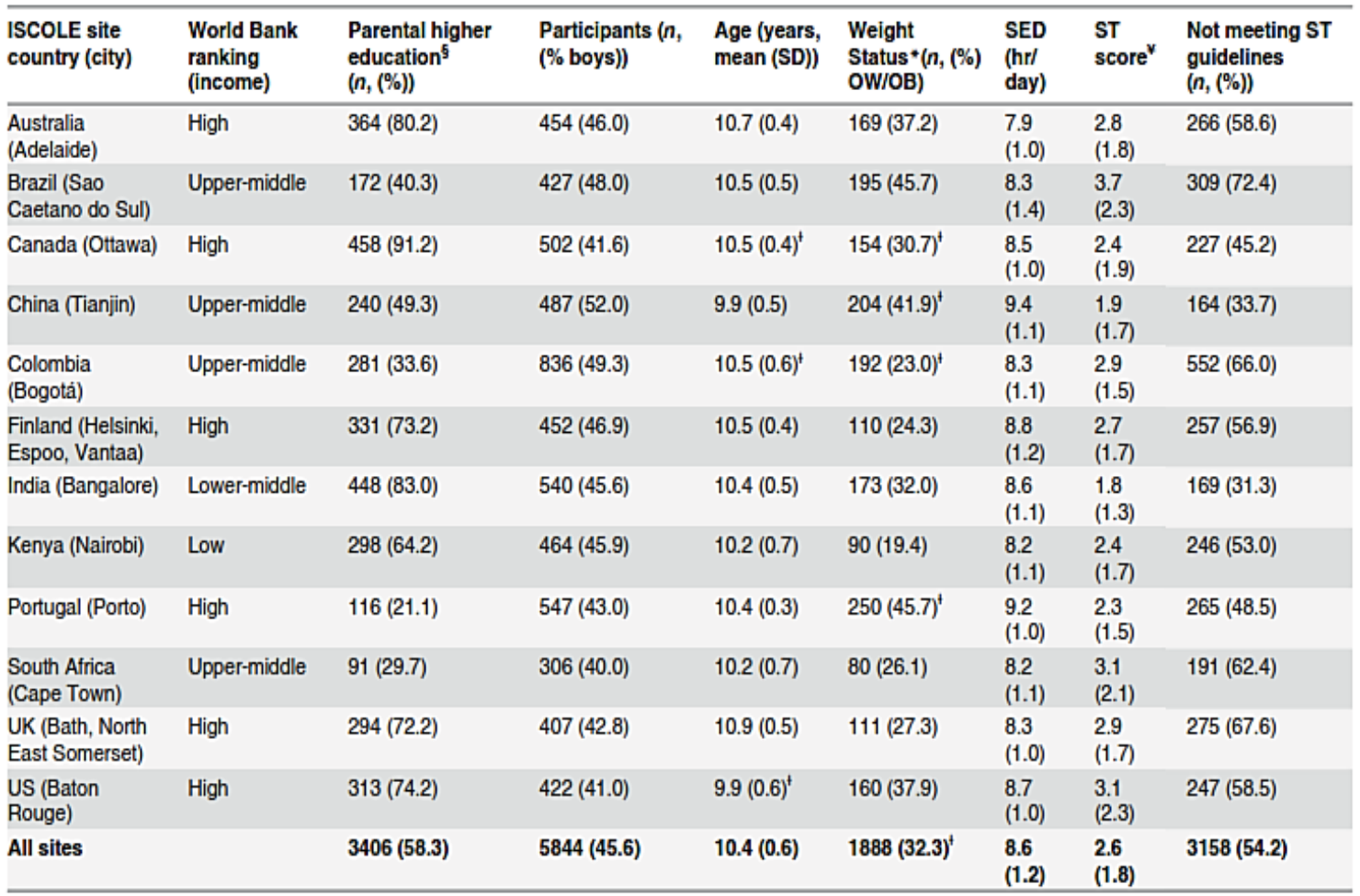

${ }^{5}$ Number $(\%)$ of sample who had at least one parent complete more than at least high school education (i.e., at least some college/university). *Number (\%) with WHO BMI Z-score classification overweight or obese

'Sites where boys had significantly higher values than girls $(p<0.05)$.

"ST score $=[$ [hours of TV on weekdays $\times 5)+($ hours of TV on weekend days $x 2)+($ hours of video games and computers on weekdays $x 5)+($ hours of video games and computers on weekend days $\times 2)] / 7$

${ }^{\ddagger}$ Number (\%) of children not meeting guidelines for $\leq 2$ hours of screen time/day, in all sites, girls were significantly more likely to meet guidelines than boys $(p<0.05)$.

BMI: Body Mass Index; SED: sedentary time; SD: standard deviation; ST: screen time; UK: United Kingdom; US: United States; OW/OB: overweight/ obese.

doi:10.1371/journal.pone.0129622 t002

$(13.0 \%$ and $9.2 \%)$ and schools $(9.1 \%$ and $7.1 \%)$. Table 2 presents the means and frequencies of the descriptive variables.

Fig 2 shows the mean SED (Panel A), and ST scores (Panel B) across all ISCOLE sites. Overall, boys had higher ST scores (mean difference $=0.57$ ), were less likely to meet ST guidelines (54.2\% of boys versus $68.2 \%$ of girls), and tended to have higher BMI z-scores (mean difference $=0.16$ ) than girls. In several study sites $(9 / 12)$, girls engaged in significantly more SED than boys (mean difference $=0.29$ hours/day). China, Portugal, Finland, the US, and Canada had higher levels of SED than the ISCOLE average ( 8.6 hours/day). Brazil, the US, South Africa, Finland, the UK, Colombia, and Australia had higher ST scores than the ISCOLE average (2.6 hours/day). India had the highest percent $(68.7 \%)$, and Brazil had the lowest percent $(27.6 \%)$ of participants meeting ST guidelines ( $\leq 2$ hours per day) [22]. 

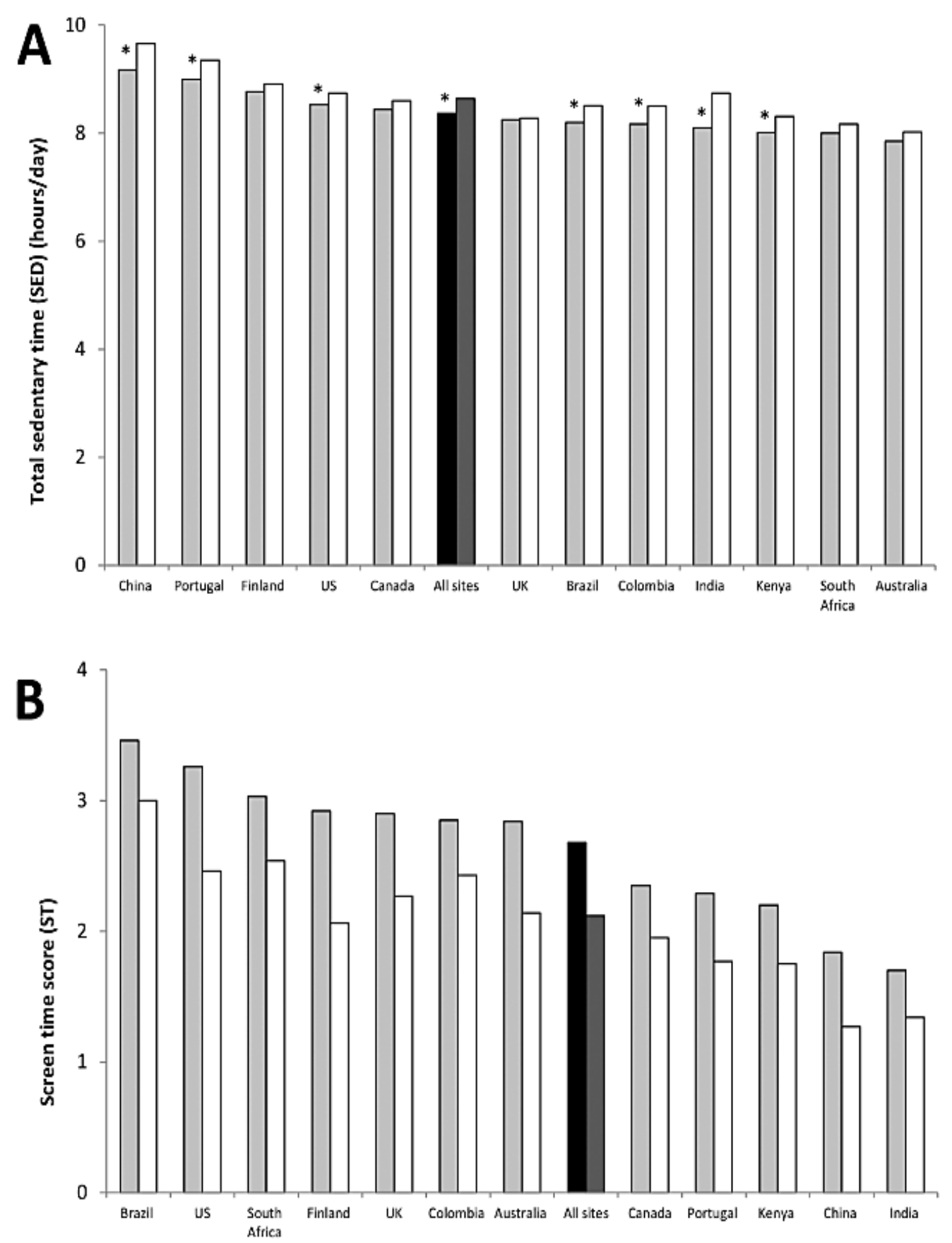

Fig 2. Mean total sedentary time (SED) and screen time (ST) score. Mean accelerometer measured SED (Panel A) and self-reported ST (Panel B) for boys (light grey bars), and girls (white bars). Black bars (boys) and dark grey bars (girls) represent overall sample means. Panel A: Accelerometer measured total sedentary time (SED) (hours/day) across all 12 ISCOLE sites (*indicates sites where girls engaged in significantly more SED than boys, $p<0.05$ ). Panel B: Self-reported screen time (ST) score across all 12 ISCOLE country sites (in all sites boys had significantly higher values for ST than girls, $p<0.05$ ).

doi:10.13715journal.pone.0129622.g002 


\section{Univariate Analyses}

The results of the univariate regression analyses are presented in Tables 3 and 4 . Of the 21 potential biological, behavioral, parental, home, and neighborhood correlates, 13 were significantly associated with SED in the total sample (14 for boys, and 12 for girls) and 17 were significantly associated with ST in the total sample ( 16 for boys, and 18 for girls).

\section{Multivariable Analyses}

Results of the final multivariable regression models are presented in Table 5. In the final models, 7 correlates of SED ( 8 in boys and 5 in girls) and 10 correlates of ST ( 10 in boys and 10 in girls) were identified. This included four common correlates across the total sample: adiposity

Table 3. Univariate correlates of total sedentary time (SED) $(n=5,844)^{\mathrm{a}}$.

\begin{tabular}{|c|c|c|c|c|c|c|c|c|c|}
\hline Variables & $\begin{array}{l}\text { Total } \\
\beta \text {-coefficient }\end{array}$ & SE & p-value & $\begin{array}{l}\text { Boys } \\
\beta \text {-coefficient }\end{array}$ & SE & p-value & $\begin{array}{l}\text { Girls } \\
\beta \text {-coefficient }\end{array}$ & SE & p-value \\
\hline \multicolumn{10}{|l|}{ Biological } \\
\hline Percent body fat ${ }^{b}$ & 0.83 & 0.11 & $<0.0001$ & 1.10 & 0.18 & $<0.0001$ & 0.71 & 0.15 & $<0.0001$ \\
\hline Waist circumference & 0.63 & 0.09 & $<0.0001$ & 0.76 & 0.14 & $<0.0001$ & 0.55 & 0.13 & $<0.0001$ \\
\hline BMI z-score ${ }^{\circ}$ & 2.71 & 0.67 & $<0.0001$ & 3.02 & 1.00 & 0.0026 & 2.86 & 0.90 & 0.0014 \\
\hline \multicolumn{10}{|l|}{ Behavioral } \\
\hline Unhealthy eating pattem score & -6.50 & 1.01 & $<0.0001$ & -6.37 & 1.46 & $<0.0001$ & -6.35 & 1.42 & $<0.0001$ \\
\hline Healthy eating pattern score & -2.81 & 0.86 & 0.0011 & -2.92 & 1.33 & 0.0282 & -2.58 & 1.14 & 0.0242 \\
\hline Passive commute to school & -3.62 & 2.02 & 0.0744 & -6.59 & 3.01 & 0.0289 & -2.25 & 2.67 & 0.3991 \\
\hline Poor sleep quality & 4.88 & 3.06 & 0.1108 & 8.07 & 4.62 & 0.0809 & 1.95 & 4.13 & 0.6377 \\
\hline Poor sleep quantity & 2.85 & 2.77 & 0.3024 & 6.81 & 4.05 & 0.0933 & 0.21 & 3.84 & 0.9561 \\
\hline Not meeting PA guidelines & 10.33 & 2.07 & $<0.0001$ & 14.89 & 3.05 & $<0.0001$ & 5.63 & 2.83 & 0.0472 \\
\hline$<2$ hours outside before school & 11.31 & 3.13 & 0.0003 & 17.61 & 4.74 & 0.0002 & 6.61 & 4.16 & 0.1120 \\
\hline$<2$ hours outside after school & 13.44 & 1.73 & $<0.0001$ & 17.13 & 2.66 & $<0.0001$ & 10.28 & 2.268 & $<0.0001$ \\
\hline$<2$ hours outside weekend & 7.47 & 1.89 & $<0.0001$ & 9.99 & 3.04 & 0.0010 & 5.67 & 2.41 & 0.0185 \\
\hline \multicolumn{10}{|l|}{ Parental characteristics } \\
\hline Mother's weight status & 1.71 & 1.69 & 0.3110 & 0.99 & 2.62 & 0.7083 & 1.80 & 2.21 & 0.4167 \\
\hline Father's weight status & 3.28 & 1.66 & 0.0471 & 2.57 & 2.57 & 0.3170 & 3.95 & 2.16 & 0.0679 \\
\hline Parental work & 1.33 & 1.70 & 0.4312 & 1.69 & 2.72 & 0.5354 & 0.39 & 2.27 & 0.8639 \\
\hline \multicolumn{10}{|l|}{ Home and neighborhood } \\
\hline Number of TVs in home & 0.53 & 2.00 & 0.7872 & 0.36 & 3.06 & 0.9060 & -0.66 & 2.56 & 0.7963 \\
\hline TV in bedroom (child-report) & 3.93 & 1.89 & 0.0372 & 2.34 & 2.87 & 0.4139 & 4.64 & 2.50 & 0.0636 \\
\hline TV in bedroom (parent-report) & 1.83 & 1.89 & 0.3360 & 7.24 & 2.88 & 0.0119 & -2.62 & 2.51 & 0.2964 \\
\hline Computer in bedroom (parent-report) & 9.17 & 1.94 & $<0.0001$ & 13.64 & 2.98 & $<0.0001$ & 6.07 & 2.56 & 0.0174 \\
\hline Automobile ownership & -4.07 & 2.81 & 0.1469 & 6.52 & 4.33 & 0.1321 & -12.97 & 3.68 & 0.0004 \\
\hline Trusted neighborhood & -1.70 & 2.20 & 0.4399 & -2.94 & 3.38 & 0.3842 & -1.03 & 2.93 & 0.7243 \\
\hline High crime neighborhood & 2.15 & 2.00 & 0.2798 & 0.64 & 3.14 & 0.8384 & 3.11 & 2.54 & 0.2218 \\
\hline Walkable neighborhood & -0.95 & 1.75 & 0.5863 & 1.07 & 2.72 & 0.6932 & -2.84 & 2.28 & 0.2120 \\
\hline
\end{tabular}

a Multi-level general linear model controlling for sex, age, and highest parental education, with school and ISCOLE site as a random effects; unstandardized beta coefficients and standard errors are presented.

'ISCOLE used a variety of measures to assess adiposity, all of which were significant in univariate analyses. Akaike information criterion, Bayesian information criterion, and level of significance were used to determine which measure of adiposity provided the closest fit for the data. Percent body fat alone provided the best fit.

'WHO BMI z-score classification. PA: physical activity; SE: Standard error; TV: television

doi:10.137115ournal.pone. 0129622 to03 
Table 4. Univariate correlates of total screen time (ST) $(n=5,844)^{\mathrm{a}}$.

\begin{tabular}{|c|c|c|c|c|c|c|c|c|c|}
\hline Variables & $\begin{array}{l}\text { Total } \\
\boldsymbol{\beta} \text {-coefficient }\end{array}$ & SE & p-value & $\begin{array}{l}\text { Boys } \\
\beta \text {-coefficient }\end{array}$ & SE & $p$-value & $\begin{array}{l}\text { Girls } \\
\beta \text {-coefficient }\end{array}$ & SE & p-value \\
\hline \multicolumn{10}{|l|}{ Biological } \\
\hline Percent body fat & 0.004 & 0.001 & $<0.0001$ & 0.004 & 0.001 & 0.0013 & 0.004 & 0.001 & 0.0001 \\
\hline Waist circumference ${ }^{b}$ & 0.003 & 0.001 & $<0.0001$ & 0.003 & 0.001 & 0.0022 & 0.004 & 0.001 & $<0.0001$ \\
\hline BMl z-score ${ }^{\circ}$ & 0.02 & 0.01 & $<0.0001$ & 0.03 & 0.01 & 0.0002 & 0.02 & 0.01 & 0.0002 \\
\hline \multicolumn{10}{|l|}{ Behavioral } \\
\hline Unhealthy eating pattem score & 0.14 & 0.01 & $<0.0001$ & 0.14 & 0.01 & $<0.0001$ & 0.15 & 0.01 & $<0.0001$ \\
\hline Healthy eating pattern score & -0.06 & 0.01 & $<0.0001$ & -0.05 & 0.01 & $<0.0001$ & -0.07 & 0.01 & $<0.0001$ \\
\hline Passive commute to school & 0.02 & 0.02 & 0.2468 & 0.00 & 0.02 & 0.8717 & 0.04 & 0.02 & 0.0463 \\
\hline Poor sleep quality & 0.09 & 0.02 & $<0.0001$ & 0.07 & 0.03 & 0.0352 & 0.11 & 0.03 & 0.0008 \\
\hline Poor sleep quantity & 0.08 & 0.02 & $<0.0001$ & 0.08 & 0.03 & 0.0072 & 0.09 & 0.03 & 0.0032 \\
\hline Not meeting PA guidelines & 0.04 & 0.02 & 0.0173 & 0.03 & 0.02 & 0.1599 & 0.05 & 0.02 & 0.0368 \\
\hline$<2$ hours outside before school & -0.02 & 0.02 & $<0.0001$ & -0.21 & 0.03 & $<0.0001$ & -0.19 & 0.03 & $<0.0001$ \\
\hline$<2$ hours outside after school & -0.08 & 0.01 & $<0.0001$ & -0.07 & 0.02 & $<0.0001$ & -0.08 & 0.02 & $<0.0001$ \\
\hline$<2$ hours outside weekend & -0.10 & 0.01 & $<0.0001$ & -0.11 & 0.02 & $<0.0001$ & -0.10 & 0.20 & $<0.0001$ \\
\hline \multicolumn{10}{|l|}{ Parental characteristics } \\
\hline Mother's weight status & 0.03 & 0.01 & 0.0071 & 0.07 & 0.02 & $<0.0001$ & 0.01 & 0.02 & 0.5919 \\
\hline Father's weight status & 0.01 & 0.01 & 0.5097 & 0.03 & 0.02 & 0.1500 & -0.01 & 0.02 & 0.5696 \\
\hline Parental work & 0.00 & 0.01 & 0.9713 & 0.01 & 0.02 & 0.5257 & -0.01 & 0.02 & 0.7826 \\
\hline \multicolumn{10}{|l|}{ Home and neighborhood } \\
\hline Number of TVs in home & -0.07 & 0.01 & $<0.0001$ & -0.08 & 0.02 & 0.0004 & -0.08 & 0.02 & $<0.0001$ \\
\hline TV in bedroom (child-report) & 0.16 & 0.01 & $<0.0001$ & 0.20 & 0.02 & $<0.0001$ & 0.13 & 0.02 & $<0.0001$ \\
\hline TV in bedroom (parent-report) & 0.11 & 0.01 & $<0.0001$ & 0.14 & 0.02 & $<0.0001$ & 0.10 & 0.02 & $<0.0001$ \\
\hline Computer in bedroom (parent-report) & 0.07 & 0.01 & $<0.0001$ & 0.09 & 0.02 & $<0.0001$ & 0.05 & 0.02 & 0.0130 \\
\hline Automobile ownership & 0.06 & 0.02 & 0.0045 & 0.06 & 0.03 & 0.0688 & 0.06 & 0.03 & 0.0434 \\
\hline Trusted neighborhood & 0.01 & 0.02 & 0.7502 & 0.02 & 0.02 & 0.4567 & 0.04 & 0.02 & 0.8572 \\
\hline High crime neighborhood & 0.00 & 0.01 & 0.8595 & 0.00 & 0.02 & 0.8237 & -0.01 & 0.02 & 0.3221 \\
\hline Walkable neighborhood & -0.02 & 0.01 & 0.1416 & -0.02 & 0.02 & 0.3283 & -0.02 & 0.01 & $<0.0001$ \\
\hline
\end{tabular}

aulti-level general linear model controlling for sex, age, and highest parental education, with school and ISCOLE site as a random effects; unstandardized beta coefficients and standard errors are presented.

"ISCOLE used a variety of measures to assess adiposity, all of which were significant in univariate analyses. Akaike information criterion, Bayesian information criterion, and level of significance were used to determine which measure of adiposity provided the closest fit for the data. Waist circumference alone provided the best fit.

'WHO BMI z-score classification. PA: physical activity; SE: Standard error, TV: television

doi:10.1371/journal.pone.0129622 t004

(percent body fat for SED and waist circumference for ST), TV in the bedroom (child-reported), computer in the bedroom, and meeting physical activity guidelines. To show significant correlates across sites, we ran the final models for SED and ST in each site country separately (keeping school as a random effect). Table 6 shows significant correlates (and their direction) in each ISCOLE site. For total SED, correlates that were most common across sites were percent body fat (positive association), and unhealthy eating pattern score (negative association). For ST, correlates that were most common across sites were healthy (negative association) and unhealthy (positive association) eating pattern scores.

Total sedentary time. Across the total sample, SED was positively associated with percent body fat, not meeting physical activity guidelines, time outside after school, TV in the bedroom (parent-reported), and computer in the bedroom. SED was negatively associated 
Table 5. Final model for correlates of accelerometer measured total sedentary time (SED) and self-reported screen time (ST) ( $n=5,844)$.

\begin{tabular}{|c|c|c|c|c|c|c|c|c|c|}
\hline \multirow[b]{2}{*}{ Variables } & \multicolumn{3}{|c|}{ Total } & \multicolumn{3}{|c|}{ Boys } & \multicolumn{3}{|c|}{ Girls } \\
\hline & $\beta$-coefficient & SE & p-value & $\beta$-coefficient & SE & p-value & $\beta$-coefficient & SE & p-value \\
\hline \multicolumn{10}{|l|}{ Final model for SED ${ }^{a}$} \\
\hline Percent body fat & 0.76 & 0.11 & $<0.0001$ & 0.98 & 0.18 & $<0.0001$ & 0.68 & 0.15 & $<0.0001$ \\
\hline Father's weight status & -1.49 & 1.66 & 0.3702 & 0.38 & 2.55 & 0.8802 & -2.70 & 2.18 & 0.2168 \\
\hline TV in bedroom (child-report) & -7.10 & 2.40 & 0.0031 & -9.75 & 3.58 & 0.0065 & -4.18 & 3.24 & 0.1967 \\
\hline TV in bedroom (parent-report) & 5.12 & 2.41 & 0.0337 & 10.11 & 3.60 & 0.0050 & -0.22 & 3.25 & 0.9468 \\
\hline Computer (parent-report) & 8.57 & 1.96 & $<0.0001$ & 11.99 & 3.00 & $<0.0001$ & 6.30 & 2.59 & 0.0150 \\
\hline Car ownership & -3.15 & 2.79 & 0.2592 & 7.13 & 4.26 & 0.0943 & -12.37 & 3.68 & 0.0008 \\
\hline Unhealthy eating pattern Score & -5.05 & 1.03 & $<0.0001$ & -4.57 & 1.47 & 0.0019 & -5.03 & 1.44 & 0.0005 \\
\hline Not meeting PA guidelines & 8.01 & 3.16 & 0.0001 & 11.52 & 3.06 & 0.0002 & 3.95 & 2.86 & 0.1679 \\
\hline$<2$ hours outside before school & 5.76 & 3.16 & 0.0683 & 12.54 & 4.77 & 0.0085 & 1.20 & 4.21 & 0.7748 \\
\hline$<2$ hours outside after school & 10.81 & 1.76 & $<0.0001$ & 12.99 & 2.70 & $<0.0001$ & 8.83 & 2.33 & 0.0001 \\
\hline \multicolumn{10}{|l|}{ Final model for ST } \\
\hline Waist circumference & 0.003 & 0.001 & $<0.0001$ & 0.003 & 0.001 & 0.0020 & 0.004 & 0.001 & $<0.0001$ \\
\hline Mother weight status & 0.02 & 0.01 & 0.1104 & 0.05 & 0.02 & 0.0053 & -0.00 & 0.02 & 0.9279 \\
\hline Number of TVs in the home & -0.03 & 0.01 & 0.0317 & -0.02 & 0.02 & 0.2553 & -0.05 & 0.02 & 0.0167 \\
\hline TV in bedroom (child-report) & 0.10 & 0.01 & $<0.0001$ & 0.13 & 0.02 & $<0.0001$ & 0.07 & 0.02 & 0.0001 \\
\hline Computer (parent-report) & 0.04 & 0.01 & 0.0022 & 0.06 & 0.02 & 0.0039 & 0.03 & 0.02 & 0.1102 \\
\hline Car ownership & 0.02 & 0.02 & 0.2279 & 0.02 & 0.03 & 0.4503 & 0.02 & 0.03 & 0.4354 \\
\hline Unhealthy eating pattern score & 0.13 & 0.01 & $<0.0001$ & 0.12 & 0.01 & $<0.0001$ & 0.14 & 0.01 & $<0.0001$ \\
\hline Healthy eating pattern score & -0.07 & 0.01 & $<0.0001$ & -0.06 & 0.01 & $<0.0001$ & -0.07 & 0.01 & $<0.0001$ \\
\hline Not meeting PA guidelines & 0.04 & 0.01 & 0.0044 & 0.03 & 0.02 & 0.1047 & 0.05 & 0.02 & 0.0111 \\
\hline$<2$ hours outside before school & -0.15 & 0.02 & $<0.0001$ & -0.14 & 0.03 & $<0.0001$ & -0.15 & 0.03 & $<0.0001$ \\
\hline$<2$ hours outside after school & -0.04 & 0.01 & 0.0012 & -0.04 & 0.02 & 0.0308 & -0.04 & 0.02 & 0.0181 \\
\hline$<2$ hours outside on weekends & -0.09 & 0.01 & $<0.0001$ & -0.09 & 0.02 & $<0.0001$ & -0.08 & 0.02 & $<0.0001$ \\
\hline
\end{tabular}

aMulti-level general linear model controlling for sex, age, and highest parental education, with school and ISCOLE site as a random effects; unstandardized beta coefficients and standard errors are presented. PA: physical activity; SE: Standard Error; SED: accelerometer measured sedentary time; ST: self-reported screen time; TV: television

doi:10.13715ournal.pone.0129622 t005

with unhealthy eating pattern score, and TV in the bedroom (child-reported). In other words, children who were more sedentary had higher body fat, were less active, spent less time outside after school, and had a computer, and a TV in their bedroom (via parental report). Children who were less sedentary ate more unhealthy food, and self-reported that they didn't have a TV in their bedroom.

For boys, SED was positively associated with percent body fat, not meeting physical activity guidelines, time outside before, or after school, TV in the bedroom (parent-reported), and computer in the bedroom; SED was negatively associated with unhealthy eating pattern score, and TV in the bedroom (child-reported). That is to say, when looking at just boys, those who were more sedentary, had higher body fat, were less active, spent less time outside, and had a computer and a TV in their bedroom (via parental report). Boys who were less sedentary ate more unhealthy food, and self-reported that they didn't have a TV in their bedroom.

For girls, SED was positively associated with percent body fat, computer in the bedroom, and spending less than 2 hours outside after school; SED was negatively associated with household car ownership, and unhealthy eating pattern score. So, when just looking at girls, those who were more sedentary had higher body fat, spent less time outside, and had a computer in 
Table 6. Significant correlates by ISCOLE country site for total sedentary time (SED) and screen time (ST).

\begin{tabular}{|c|c|c|c|c|c|c|c|c|c|c|c|c|}
\hline & Australia & Brazil & Canada & China & Colombia & Finland & India & Kenya & Portugal & $\begin{array}{l}\text { South } \\
\text { Africa }\end{array}$ & $\begin{array}{l}\text { U. } \\
\text { K. }\end{array}$ & $\begin{array}{l}\text { U. } \\
\text { S. }\end{array}$ \\
\hline \multicolumn{13}{|l|}{ Final model for SED } \\
\hline Percent body fat & + & & + & & + & + & & & & & + & + \\
\hline Father weight status & & - & & & & & & & & & & \\
\hline TV (child-report) & & & & & & & & + & & - & & \\
\hline TV (parent-report) & & & & - & & & & & & + & & \\
\hline Computer (parent-report) & & & & + & & + & & & & & + & \\
\hline Car ownership & & & & - & & & & & & & & \\
\hline $\begin{array}{l}\text { Unhealthy eating pattem } \\
\text { score }\end{array}$ & & - & - & - & - & & & & & & & \\
\hline Not meeting PA guidelines & + & & & & & + & & & & & & + \\
\hline $\begin{array}{l}<2 \text { hours outside before } \\
\text { school }\end{array}$ & & & & & & + & & & & & & \\
\hline$<2$ hours outside after school & + & + & & & + & + & & & + & + & + & + \\
\hline \multicolumn{13}{|l|}{ Final model for $\mathrm{ST}^{\mathrm{b}}$} \\
\hline Waist circumference & + & & + & & + & + & + & & & & + & \\
\hline Mother weight status & - & & & & & & & & & & & \\
\hline Number of TVs in the home & & & - & & & & + & & & & & \\
\hline TV (child-report) & & & & + & & & & & + & & + & + \\
\hline Computer (parent-report) & & + & + & & & & & & + & & & \\
\hline \multicolumn{13}{|l|}{ Car ownership } \\
\hline $\begin{array}{l}\text { Unhealthy eating pattem } \\
\text { score }\end{array}$ & + & + & + & + & + & + & + & + & + & + & + & + \\
\hline Healthy eating pattern score & - & & - & - & - & - & - & & - & & & - \\
\hline Not meeting PA guidelines & & & & & & & + & & & & & \\
\hline $\begin{array}{l}<2 \text { hours outside before } \\
\text { school }\end{array}$ & & & - & & & & - & - & & - & & \\
\hline$<2$ hours outside after school & & & & - & & & & & & - & & \\
\hline $\begin{array}{l}<2 \text { hours outside on } \\
\text { weekends }\end{array}$ & & & & - & & & - & - & - & & & \\
\hline
\end{tabular}

aMulti-level general linear model controlling for sex, age, and highest parental education, with school as a random effect. $(+)$ indicates a direct relationship between SED or ST and the associated correlate; (-) indicates an inverse relationship between SED or ST and the associated correlate. A blank cell indicates no significant association was found for the particular correlate in the respective country. PA: physical activity; SED: accelerometer measured sedentary time; ST: self-reported screen time; TV: television; U.K.: United Kingdom; U.S.: United States.

doi:10.1371 ijournal.pone.0129622 t006

their bedroom. Girls who were less sedentary ate more unhealthy food, and had higher household car ownership.

Screen time. Across the total sample, ST was positively associated with waist circumference, TV in the bedroom (child-reported), computer in the bedroom, unhealthy eating pattern score, and meeting physical activity guidelines. ST was negatively associated with number of TVs in the house, healthy eating pattern score, and spending $<2$ hours/day outside before school, after school, or on weekends. In other words, for the whole sample, children who reported more ST were less active, ate more junk food, and were more likely to have a TV and a computer in their bedroom. Children who reported less ST had a healthier diet, spent less time outside, and had more TVs in their house.

For ST, correlates for boys and girls alone were the same as those of the total sample, with a few exceptions. Correlates for boys were the same as those identified for the total sample except 
we saw no significant association with ST and number of TVs in the house. For girls, correlates of ST also included household car ownership, and did not include having a computer in their bedroom.

For analysis of SED, potential correlates (except weight status and healthy/unhealthy eating pattern scores) were re-coded as dichotomous variables. The advantage of this analytical approach was that all variables were on approximately the same scale, so that the strength of the association of each correlate could be examined, and interpreted as a proportional increase in the outcome variable (e.g., a unit increase in the beta coefficient for a correlate of SED represented an increase of one minute/day of SED). This information can provide guidance as to which correlates should be targeted in future work. The correlates that showed the strongest association with higher SED were time outside after school ( $\geq 2$ hours), having a computer in the bedroom (parent-report), and not meeting physical activity guidelines (child-report). These variables represented approximately 10.8 minutes, 8.6 minutes, and 8.0 minutes of SED, respectively. Because ST was measured as a score, instead of hours/day, it could not be interpreted the same way.

\section{Discussion}

This study aimed to identify biological, behavioral, parental, home, and neighborhood correlates of SED, and ST in 10 year-old children from study sites in 12 countries around the world. To date, this represents the most geographically and culturally diverse study sample, and is one of few studies to examine both objectively measured SED, and self-reported ST in the same population. We were able to identify four common correlates of SED and ST (adiposity, TV in the bedroom, computer in the bedroom, and time outside after school). Overall, we were able to identify a greater number of correlates for ST than for SED, and many of the correlates we identified were the same among boys and girls.

Many correlates identified here are consistent with previous work [15]. Previous work has consistently shown that boys engage in more ST, and are less likely to meet ST guidelines than girls [15,30-32], while girls accumulate more SED than boys [15,33]. Many studies have also linked higher levels of sedentarism with greater body weight or adiposity, and with availability of media equipment in the home [15]. It is interesting that we found different results for TV in the bedroom depending on whether the question was answered by the child, or the parent. Previous work has shown that having a TV in the bedroom is associated with higher ST [15], greater risk for obesity [34], higher cardiometabolic risk [35], lower physical activity [36], and shorter self-reported sleep [37]. A possible explanation is social-desirability bias of either the children (i.e., who want to show-off), or the parents (i.e., who know that TV in the bedroom is bad). It is also possible that there was some misunderstanding since many people use a computer or a tablet to watch TV programming, and did or did not count a computer screen as a having TV in the bedroom. This may also help to explain why having a computer in the bedroom was associated with such a large effect on SED (approximately 8.6 minutes/day more than not having a TV in the bedroom). In previous work, Roemmich et al., reported that greater ST was associated with the number of TVs in the home for girls, but not boys [38]. This is consistent with our results; however, we saw that having more TVs in the home was negatively associated with ST score. Possible explanations for this include the placement of the TVs (e.g., the TV could be in the parent's bedroom, and not accessible for the child), or parental rules regarding TV usage (e.g., one hour per evening).

Consistent with previous work, we saw that greater outdoor time was associated with lower SED [39]; however, we also saw that greater outdoor time (before school, after school, and on weekends) was associated with a higher ST score. To explain this relationship, we ran the final 
model for ST score, adjusting for accelerometer measured moderate- to vigorous-intensity physical activity, and then again adjusting for SED, but the association remained significant in both instances. It is possible that children who spent more time outside were compensating with higher ST when they were inside. This warrants further exploration. Another perplexing finding was the relationship with SED and unhealthy eating pattern score. One would assume that higher unhealthy eating pattern scores would be associated with higher levels of SED and ST (e.g., those who were more sedentary, or watched more TV, ate more unhealthy food). We found that this relationship was true for the relationship between ST score and unhealthy eating pattern score; however, our analysis showed that lower SED was associated with higher unhealthy eating pattern score (i.e., children who were less sedentary ate more junk food). The reason for this is not obvious and warrants further exploration.

Throughout this paper, we used self-reported physical activity instead of using accelerometer derived moderate- to vigorous-intensity physical activity. This is because there is debate as to the appropriateness of including more than one movement variable in the same model. Although the movement variables may not be highly correlated (i.e., no issues with multicollinearity), they are still dependent on each other since they are derived from the same measurement device (i.e. proportional error). Including accelerometer-derived moderate- to vigorousintensity physical activity variables can also mask variance that could be attributed to other potential correlates. We did examine this to determine if changed the significance of our correlates. For SED, the correlates did not change, with the addition of household car ownership that became significant $(\mathrm{p}=0.03)$. For $\mathrm{ST}$, all correlates remained the same.

To examine how correlates might differ across ISCOLE sites, and to help inform public health strategies and messaging, final models were examined for each country. Overall, when significant, the direction of the association was consistent across sites. This is especially interesting given the large geographical, socio-economical, and culturally diverse sample. Percent body fat and time outside after school were the most common correlates of SED, and eating pattern scores (healthy and unhealthy) were the most common correlates of ST. This information can be used to provide harmonized, as well as country-specific, public health messages in different countries.

Until recently, sedentary behavior research has relied on self-reported measures, and has primarily examined screen-based behaviors. With the widespread use of accelerometers, we are now able to capture SED throughout the day; however, high levels of SED are not always a good predictor of high ST. For example, in ISCOLE, participants from the China site engaged in the highest amount of SED ( 9.4 hours/day), but had the second lowest ST score (approximately 1.9 hours/day). Similarly, boys reported more ST than girls across all sites, but in the majority of sites, girls engaged in more SED. Across the whole sample, self-reported ST score explained only a small portion (approximately $30 \%$ ) of accelerometer measured SED. This is consistent with previous work that suggests that although ST is an important aspect of sedentary time, it may not be a good marker for SED [6], and highlights the idea that researchers should be examining a variety of sedentary pursuits.

While accelerometers are able to provide data related to movement/non-movement patterns for the whole day, they are not able to discriminate between different postures (e.g., standing still versus sitting still), or types of sedentary behaviors (e.g., reading versus watching TV) [40]. This suggests that there is a large proportion of daily SED that is unaccounted for by questioning on ST alone. Results from this work suggest that current sedentary behavior guidelines for children and youth that recommend minimizing all SED [22] could be clarified by focusing specifically on reducing ST, with future work aimed at understanding related health effects (both negative and positive) of other, non-screen based sedentary behaviors (e.g., reading a book, coloring). Future research should also try and disentangle sedentary multi-tasking (e.g., 


\section{象PLOS $\left.\right|_{\text {ONE }}$}

watching $\mathrm{TV}$, while texting on a smartphone, and surfing the internet) and the related health effects.

Finally, it is important to note that although our findings are supported by previous work, with the emergence of sedentary behavior research, it is possible that not all studies have used the same definitions, or cut-points, for SED or ST that we have used here. The difference in measurement procedures, cut-points used or definitions of SED and/or ST may explain some of the differences in results that have been presented.

\section{Strengths and Limitations}

The work presented here was restricted to children 9-11 years of age and therefore limits the generalizability to other age groups. However, while TV viewing and other screen-based sedentary behaviors are expected to change with age, it has been suggested that there is not sufficient rationale to assume correlates of SED and ST vary also [14]. ISCOLE also relied on child-reported ST; it would have been beneficial to ask both the parents and the children to report on time spent engaging in ST to understand their unique perspectives. Parental report could also provide additional information on parent-related behaviors (e.g., parental ST habits, household rules for ST), giving additional insight into the home environment. ST in ISCOLE is also limited to recreational screen-based activities and does not examine other sedentary behaviors such as reading or drawing (for pleasure or for school). This could be ameliorated by using time-use surveys; however, time-use surveys require additional time to administer and are not feasible for population-based studies [7]. Finally, this work relied largely on child-report questionnaires, which don't necessarily provide an accurate reflection of the true situation.

The major strengths of ISCOLE are associated with the overall study design and administration [16]. ISCOLE is the most culturally and geographically diverse, up-to-date, and robust study on lifestyles associated with obesity-related health in children. The ISCOLE framework and coordinating center ensured all study sites, and all ISCOLE researchers, completed mandatory training for all aspects of the study. ISCOLE also made use of direct measurement for all anthropometric variables, and accelerometers for all activity variables. Accelerometers have been shown to be a valid tool to measure movement at all levels of intensity, and the cut-points used in this analysis have been shown to have a high sensitivity for physical activity and for SED [19]. It is possible that the use of inclinometers, rather than traditional accelerometers may better capture time spent in SED, but they are more obtrusive to attach to the participant and generally considered uncomfortable to wear for long time periods [7].

\section{Conclusion}

Many common correlates of SED and ST were identified in this large, global, and culturally and socioeconomically diverse sample. Some of these are easily modifiable (e.g., removing a TV or a computer from the bedroom), and others may require more intense behavioral interventions (e.g., increasing habitual physical activity to meet current guidelines). The results of this study support the idea that a single strategy to reduce SED and ST is unlikely to be effective across many countries; however, a strategy aimed at similar behaviours (i.e., correlates identified here), with country-specific interventions, is possible. Future work should adapt these findings to provide culturally meaningful public health strategies and messages and test these correlates in a multi-faceted intervention to reduce SED and ST in children around the world. This may help to improve lifestyle behaviors such as physical activity, reduce excessive time spent in SED and ST, and ultimately reduce the risk of preventable chronic diseases such as obesity worldwide.

PLOS ONE|DOI:10.1371/journal.pone.0129622 June 11, 2015 $16 / 20$ 


\section{Acknowledgments}

We wish to thank the ISCOLE External Advisory Board and the ISCOLE participants and their families who made this study possible. The ISCOLE Research Group includes: Coordinating Center, Pennington Biomedical Research Center: Peter T. Katzmarzyk, PhD (Co-PI), Timothy S. Church, MD, PhD (Co-PI), Denise G. Lambert, RN (Project Manager), Tiago Barreira, $\mathrm{PhD}$, Stephanie Broyles, $\mathrm{PhD}$, Ben Butitta, BS, Catherine Champagne, PhD, RD, Shannon Cocreham, MBA, Kara Dentro, MPH, Katy Drazba, MPH, Deirdre Harrington, PhD, William Johnson, $\mathrm{PhD}$, Dione Milauskas, MS, Emily Mire, MS, Allison Tohme, MPH, Ruben Rodarte MS, MBA; Data Management Center, Wake Forest University: Bobby Amoroso, BS, John Luopa, BS, Rebecca Neiberg, MS, Scott Rushing, BS; Australia, University of South Australia: Timothy Olds, PhD (Site Co-PI), Carol Maher, PhD (Site Co-PI), Lucy Lewis, PhD, Katia Ferrar, B Physio (Hon), Effie Georgiadis, BPsych, Rebecca Stanley, BAppSc (OT) Hon; Brazil, Centro de Estudos do Laboratório de Aptidão Física de São Caetano do Sul (CELAFISCS): Victor Keihan Rodrigues Matsudo, MD, PhD (Site PI), Sandra Matsudo, MD, PhD, Timoteo Araujo, MSc, Luis Carlos de Oliveira, MSc, Leandro Rezende, BSc, Luis Fabiano, BSc, Diogo Bezerra, BSc, Gerson Ferrari, MSc; Canada, Children's Hospital of Eastern Ontario Research Institute: Mark S. Tremblay, $\mathrm{PhD}$ (Site Co-PI), Jean-Philippe Chaput, $\mathrm{PhD}$ (Site Co-PI), Priscilla Bélanger, MA, Mike Borghese, MSc, Charles Boyer, MA, Allana LeBlanc, MSc, Claire Francis, M.Sc.,Geneviève Leduc, PhD; China, Tianjin Women's and Children's Health Center: Pei Zhao, MD (Site Co-PI), Gang Hu, MD, PhD (Site Co-PI), Chengming Diao, MD, Wei Li, MD, Weiqin Li, MSc, Enqing Liu, MD, Gongshu Liu, MD, Hongyan Liu, MSc, Jian Ma, MD, Yijuan Qiao, MSc, Huiguang Tian, PhD, Yue Wang, MD, Tao Zhang, MSc, Fuxia Zhang, MD; Colombia, Universidad de los Andes: Olga Sarmiento, MD, PhD (Site PI), Julio Acosta, Yalta Alvira, BS, Maria Paula Diaz, Rocio Gamez, BS, Maria Paula Garcia, Luis Guillermo Gómez, Lisseth Gonzalez, Silvia Gonzalez, RD, Carlos Grijalba, MD, Leidys Gutierrez, David Leal, Nicolas Lemus, Etelvina Mahecha, BS, Maria Paula Mahecha, Rosalba Mahecha, BS, Andrea Ramirez, MD, Paola Rios, MD, Andres Suarez, Camilo Triana; Finland, University of Helsinki: Mikael Fogelholm, ScD (Site-PI), Elli Hovi, BS, Jemina Kivelä, Sari Räsänen, BS, Sanna Roito, BS, Taru Saloheimo, MS, Leena Valta; India, St. Johns Research Institute: Anura Kurpad, MD, PhD (Site Co-PI), Rebecca Kuriyan, PhD (Site Co-PI), Deepa P. Lokesh, BSc, Michelle Stephanie D'Almeida, BSc, Annie Mattilda R, MSc, Lygia Correa, BSc, Vijay D, BSc; Kenya, Kenyatta University: Vincent Onywera, PhD (Site Co-PI), Mark S. Tremblay, PhD (Site Co-PI), Lucy-Joy Wachira, PhD, Stella Muthuri, PhD; Portugal, University of Porto: Jose Maia, PhD (Site PI), Alessandra da Silva Borges, BA, Sofia Oliveira Sá Cachada, Msc, Raquel Nichele de Chaves, MSc, Thayse Natacha Queiroz Ferreira Gomes, MSc, Sara Isabel Sampaio Pereira, BA, Daniel Monteiro de Vilhena e Santos, PhD, Fernanda Karina dos Santos, MSc, Pedro Gil Rodrigues da Silva, BA, Michele Caroline de Souza, MSc; South Africa, University of Cape Town: Vicki Lambert, PhD (Site PI), Matthew April, BSc (Hons), Monika Uys, BSc (Hons), Nirmala Naidoo, MSc, Nandi Synyanya, Madelaine Carstens, BSc(Hons); United Kingdom, University of Bath: Martyn Standage, $\mathrm{PhD}$ (Site PI), Sean Cumming, PhD, Clemens Drenowatz, PhD, Lydia Emm, MSc, Fiona Gillison, PhD, Julia Zakrzewski, PhD; United States, Pennington Biomedical Research Center: Catrine Tudor-Locke, $\mathrm{PhD}$ (Site-PI), Ashley Braud, Sheletta Donatto, MS, LDN, RD, Corbin Lemon, BS, Ana Jackson, BA, Ashunti Pearson, MS, Gina Pennington, BS, LDN, RD, Daniel Ragus, BS, Ryan Roubion, John Schuna, Jr., PhD; Derek Wiltz. The ISCOLE External Advisory Board includes Alan Batterham, $\mathrm{PhD}$, Teesside University, Jacqueline Kerr, PhD, University of California, San Diego; Michael Pratt, MD, Centers for Disease Control and Prevention, Angelo Pietrobelli, MD, Verona University Medical School. 


\section{Author Contributions}

Conceived and designed the experiments: AGL MST PTK JPC STB. Analyzed the data: AGL STB MST PTK. Wrote the paper: AGL PTK TVB STB JPC TSC MF DMH GH RK AK EVL CM JM VM TO VO OLS MS CTL PZ MST.

\section{References}

1. Janssen I, LeBlanc AG. Systematic review of the health benefits of physical activity and fitness in school-aged children and youth. Int J Behav Nutr Phys Act. 2010; 7: 40. doi: 10.1186/1479-5868-7-40 PMID: 20459784

2. LeBlanc AG, Spence JC, Carson V, Gorber SC, Dillman C, Janssen I, et al. Systematic review of sedentary behaviour and health indicators in the early years (aged 0-4 years). Appl Physiol Nutr Metab. 2012; 37: 753-772. doi: 10.1139/h2012-063 PMID: 22765839

3. Timmons BW, LeBlanc AG, Carson V, Connor Gorber S, Dillman C, Janssen I, et al. Systematic review of physical activity and health in the early years (aged 0-4 years). Appl Physiol Nutr Metab. 2012; 37: 773-792. doi: 10.1139/h2012-070 PMID: 22765840

4. Tremblay MS, LeBlanc AG, Kho ME, Saunders TJ, Larouche R, Colley RC, et al. Systematic review of sedentary behaviour and health indicators in school-aged children and youth. Int J Behav Nutr Phys Act. 2011; 8: 98. doi: 10.1186/1479-5868-8-98 PMID: 21936895

5. Sedentary Behaviour Research Networ. Letter to the Editor. Standardized use of the terms "sedentary" and "sedentary behaviours". Appl Physiol Nutr Metab. 2012; 37: 540-542. doi: 10.1139/h2012-024 PMID: 22540258

6. Biddle SJH, Gorely T, Marshall SJ. Is Television Viewing a Suitable Marker of Sedentary Behavior in Young People? Ann Behav Med. 2009; 38: 147-153. doi: 10.1007/s12160-009-9136-1 PMID: 19809858

7. Hardy LL, Hills AP, Timperio A, Cliff D, Lubans D, Morgan PJ, et al. A hitchhiker's guide to assessing sedentary behaviour among young people: Deciding what method to use. J Sci Med Sport. 2013; 16: 28-35. doi: 10.1016/j.jsams.2012.05.010 PMID:22749939

8. Saunders TJ, Chaput J-P, Goldfield GS, Colley RC, Kenny GP, Doucet E, et al. Prolonged sitting and markers of cardiometabolic disease risk in children and youth: a randomized crossover study. Metabolism. 2013; 62: 1423-1428. doi: 10.1016/j.metabol.2013.05.010 PMID: 23773981

9. Colley RC, Garriguet D, Janssen I, Wong SL, Saunders TJ, Carson V, et al. The association between accelerometer-measured patterns of sedentary time and health risk in children and youth: results from the Canadian Health Measures Survey. BMC Public Health. 2013; 13: 200. doi: 10.1186/1471-245813-200 PMID: 23497190

10. Brodersen $\mathrm{NH}$, Steptoe A, Boniface DR, Wardle J. Trends in physical activity and sedentary behaviour in adolescence: ethnic and socioeconomic differences. Br J Sports Med. 2007; 41: 140-144. doi: 10. 1136/bjsm.2006.031138 PMID: 17178773

11. Fakhouri TI, Hughes JP, Brody DJ, Kit BK, Ogden CL. PHysical activity and screen-time viewing among elementary school-aged children in the united states from 2009 to 2010. JAMA Pediatr. 2013; 167: 223-229. doi: 10.1001/2013.jamapediatrics.122 PMID: 23303439

12. Muthuri SK, Wachira L-JM, Leblanc AG, Francis CE, Sampson M, Onywera VO, et al. Temporal Trends and Correlates of Physical Activity, Sedentary Behaviour, and Physical Fitness among School-Aged Children in Sub-Saharan Africa: A Systematic Review. Int J Environ Res Public Health. 2014; 11: 3327-3359. doi: 10.3390/ijerph110303327 PMID: 24658411

13. Caspersen CJ, Pereira MA, Curran KM. Changes in physical activity patterns in the United States, by sex and cross-sectional age: Med Sci Sports Exerc. 2000; 1601-1609. doi: 10.1097/00005768200009000-00013 PMID: 10994912

14. Gorely T. Couch kids: Correlates of television viewing among youth. Int J Behav Med. 2004; 11: 152 163. PMID: 15496343

15. Temmel CSD, Rhodes R. Correlates of Sedentary Behaviour in Children and Adolescents Aged 7-18: A Systematic Review. Health Fit J Can. 2013; 6: 119-199.

16. Katzmarzyk PT, Barreira TV, Broyles ST, Champagne CM, Chaput J-P, Fogelholm M, et al. The International Study of Childhood Obesity, Lifestyle and the Environment (ISCOLE): design and methods. BMC Public Health. 2013; 13: 900. doi: 10.1186/1471-2458-13-900 PMID: 24079373

17. Trost SG, Loprinzi PD, Moore R, Pfeiffer KA. Comparison of Accelerometer Cut Points for Predicting Activity Intensity in Youth: Med Sci Sports Exerc. 2011; 43: 1360-1368. doi: 10.1249/MSS. Ob013e318206476e PMID: 21131873 
18. Colley RC, Connor Gorber S, Tremblay MS. Quality control and data reduction procedures for accelerometry-derived measures of physical activity. Health Rep. 2010;21: 63-69. PMID: 21269013

19. Evenson KR, Catellier DJ, Gill K, Ondrak KS, McMurray RG. Calibration of two objective measures of physical activity for children. J Sports Sci. 2008; 26: 1557-1565. doi: 10.1080/02640410802334196 PMID: 18949660

20. Barreira TV, Schuna JM, Mire EF, Katzmarzyk PT, Chaput J-P, Leduc G, et al. Identifying Children's Noctumal Sleep Using 24-h Waist Accelerometry. Med Sci Sports Exerc. 2014; doi: 10.1249/MSS. 0000000000000486

21. Youth Risk Behavior Surveillance System (YRBSS). In: U.S. Centers for Disease Control and Prevention. 2012.

22. Tremblay MS, LeBlanc AG, Janssen I, Kho ME, Hicks A, Murumets K, et al. Canadian Sedentary Behaviour Guidelines for Children and Youth. Appl Physiol Nutr Metab. 2011; 36: 59-64. doi: 10.1139/ H11-012 PMID: 21326378

23. Barreira TV, Staiano AE, Katzmarzyk PT. Validity assessment of a portable bioimpedance scale to estimate body fat percentage in White and African-American children and adolescents. Pediatr Obes. 2013; 8: e29-e32. doi: 10.1111/J.2047-6310.2012.00122.x PMID: 23239610

24. De Onis M, Lobstein T. Defining obesity risk status in the general childhood population: Which cut-offs should we use? Int J Pediatr Obes. 2010; 5: 458-460. doi: 10.3109/17477161003615583 PMID: 20233144

25. Currie C, Nic Gabhainn S, Godeau E, Roberts C, Smith R, Currie D, et al. Inequalities in Children's Health: HBSC International Report from the 2005/2006 Survey. [Internet]. Geneva: World Health Organization; 2008. Available: http://www.childhealthresearch.eu/riche/research/add-knowledge/HBSC $\% 20$ international\%20report\%202005-06\%20survey.pdf.

26. Tremblay MS, Warburton DER, Janssen I, Paterson DH, Latimer AE, Rhodes RE, et al. New Canadian Physical Activity Guidelines. Appl Physiol Nutr Metab. 2011; 36: 36-46. doi: 10.1139/H11-009 PMID: 21326376

27. Owen N, Sugiyama T, Eakin EE, Gardiner PA, Tremblay MS, Sallis JF. Adults' Sedentary Behavior: Determinants and Interventions. Am J Prev Med. 2011; 41: 189-196. doi: 10.1016/.amepre.2011.05.013 PMID: 21767727

28. Kenward MG, Roger JH. Small Sample Inference for Fixed Effects from Restricted Maximum Likelihood. Biometrics. 1997; 53: 983. doi: 10.2307/2533558 PMID: 9333350

29. Craney TA, Surles JG. Model-Dependent Variance Inflation Factor Cutoff Values. Qual Eng. 2002; 14 391.

30. Cao H, Qian Q, Weng T, Yuan C, Sun Y, Wang H, et al. Screen time, physical activity and mental health among urban adolescents in China. Prev Med. 2011; 53: 316-320. doi: 10.1016/j.ypmed.2011.09.002 PMID: 21933680

31. Cui Z, Hardy LL, Dibley MJ, Bauman A. Temporal trends and recent correlates in sedentary behaviours in Chinese children. Int J Behav Nutr Phys Act. 2011; 8: 1-8. doi: 10.1186/1479-5868-8-93 PMID: 21194492

32. Anderson SE, Economos CD, Must A. Active play and screen time in US children aged 4 to 11 years in relation to sociodemographic and weight status characteristics: a nationally representative cross-sectional analysis. BMC Public Health. 2008; 8: 366. doi: 10.1186/1471-2458-8-366 PMID: 18945351

33. Rodrigues AMM, Silva M, Mota J, Cumming SP, Sherar LB, Neville H, et al. Confounding effect of biologic maturation on sex differences in physical activity and sedentary behavior in adolescents. Pediatr Exerc Sci. 2010; 22: 442-453. PMID: 20814039

34. Adachi-Mejia AM, Longacre MR, Gibson JJ, Beach ML, Titus-Emstoff LT, Dalton MA. Children with a TV in their bedroom at higher risk for being overweight. Int J Obes. 2006; 31:644-651. doi: 10.1038/sj. ijo.0803455

35. Staiano AE, Harrington DM, Broyles ST, Gupta AK, Katzmarzyk PT. Television, Adiposity, and Cardiometabolic Risk in Children and Adolescents. Am J Prev Med. 2013; 44: 40-47. doi: 10.1016/j.amepre. 2012.09.049 PMID: 23253648

36. Cameron AJ, van Stralen MM, Brug J, Salmon J, Bere E, ChinAPaw MuM, et al. Television in the bedroom and increased body weight: potential explanations for their relationship among European schoolchildren. Pediatr Obes. 2013; 8: 130-141. doi: 10.1111/j.2047-6310.2012.00094.x PMID: 23239631

37. Cespedes EM, Gillman MW, Kleinman K, Rifas-Shiman SL, Redline S, Taveras EM. Television Viewing, Bedroom Television, and Sleep Duration From Infancy to Mid-Childhood. Pediatrics. 2014; 133: e1163-e1171. doi: 10.1542/peds.2013-3998 PMID: 24733878 
38. Roemmich JN, Epstein LH, Raja S, Yin L. The neighborhood and home environments: Disparate relationships with physical activity and sedentary behaviors in youth. Ann Behav Med. 2007; 33: 29-38. doi: 10.1207/s15324796abm3301_4 PMID: 17291168

39. Schaefer L, Plotnikoff RC, Majumdar SR, Mollard R, Woo M, Sadman R, et al. Outdoor Time Is Associated with Physical Activity, Sedentary Time, and Cardiorespiratory Fitness in Youth. J Pediatr. 2014; 165: 516-521. doi: 10.1016/j.jpeds.2014.05.029 PMID: 25043155

40. Atkin AJ, Gorely T, Clemes SA, Yates T, Edwardson C, Brage S, et al. Methods of Measurement in epidemiology: Sedentary Behaviour. Int J Epidemiol. 2012; 41: 1460-1471. doi: 10.1093/ije/dys118 PMID: 23045206 


\section{Chapter 5}

\section{Discussion}

Chapter 1 and Chapter 2 provided the reader with the necessary background information to understand and interpret the research presented within this dissertation (the "why"). Chapter 3 provided information on the rationale and development of the manuscripts (the "how"); and Chapter 4 provided the reader with the research manuscripts associated with this work (the "what"). This chapter aims to provide a summary of the research results presented in Chapter 4 and provide additional insight on the overall findings of this doctoral work and how the results are integrated together (the "so what"). I also aim to highlight currents gaps in knowledge and provide areas of opportunity for future research (the "now what"). Finally, I aim to provide strengths and limitations of the work presented within this dissertation and how these could be overcome in future work.

\subsection{Summary of research findings}

Included in this dissertation are two review papers that helped develop the background and rationale for this work, and three original research articles. All manuscripts included in this dissertation are closely linked and build upon each other.

Manuscript 1 aimed to gauge awareness of $\mathrm{PA}^{1}$ and SB (specifically ST) ${ }^{2}$ guidelines among children and determine if guideline awareness differed with activity levels. This study was informed by Background Manuscript $1,{ }^{3}$ which reported that awareness of current guidelines among the general population, moms, and pediatricians 
was low. ${ }^{3}$ Background Manuscript 1 also showed that to date, no study had examined children's awareness of PA or ST guidelines in Canada. ${ }^{3}$ Manuscript 1 was developed based on the assumption that public health messages to improve habitual behaviours are only beneficial if they are known to the general public, and the belief that children who were aware of current PA guidelines and/or ST guidelines would be more active and/or less sedentary than children who were unaware of the guidelines. We found that most children could identify PA guidelines, and that few children could identify ST guidelines. Contrary to our hypothesis, we showed that knowledge of guidelines was not associated with accelerometer-measured average daily PA, or self-reported average daily ST.

Background Manuscript 2 was included to help inform Manuscript 2 and Manuscript 3. Specifically, Background Manuscript 2 was developed to help support the work derived from ISCOLE ${ }^{4}$ and determine if ISCOLE participant characteristics were representative of children from the rest of that country. Manuscript 2 aimed to identify correlates of total accelerometer measured SED and self-reported ST in Canadian children using ISCOLE data. ${ }^{5}$ This manuscript was developed, in part to clarify our understanding of similarities and differences between SED and ST in the paediatric population. In Canada, children averaged 8.5 hours of SED per day and $46 \%$ exceeded ST guidelines. ${ }^{2,5}$ Multilevel modeling was used to account for the hierarchical nature of the data $^{6}$ and to identify potential correlates. Waist circumference and number of TVs in the home were identified as common correlates of increased SED and ST. While waist circumference (or any measure of weight status) may need to be addressed through a 
comprehensive, multi-dimensional intervention, reducing the number of TVs in the home may be a relatively easy recommendation to include in future public health guidelines.

Finally, Manuscript 3 was meant to build on Manuscript 2 and aimed to examine correlates of SED and ST in children from all 12 ISCOLE sites. ${ }^{4}$ This analysis was unique in that no study has used such a large, geographically, and socio-culturally diverse sample to understand children's SB. Around the world, children averaged 8.6 hours of SED per day and $56 \%$ of children exceeded current guidelines of $\leq 2$ hours of recreational ST per day. Consistent with the analytical framework used in Manuscript 2, multilevel modelling was used for analysis. ${ }^{6}$ Through this analysis, we identified four common correlates of increased SED and ST including weight status, having a TV in the bedroom, having a computer in the bedroom, and not meeting PA guidelines. Similar to the findings from Manuscript 2, this study suggests that there are some correlates of increased SED and ST that are easily modifiable (e.g., removing TV or computer from the bedroom), and others that may require more intense behavioural interventions (e.g., meeting PA guidelines, or weight status).

\subsection{Interpretations within a population health context}

This research was informed by the ecological models of health behaviour and SB as proposed by Sallis et al., ${ }^{7}$ and Owens et al. ${ }^{8}$ Ecological models suggest that individual behaviours are influenced by many different and sometimes, competing factors, and that interventions to change behaviours must be as multi-dimensional as the behaviours themselves. As seen in Manuscript 2 and Manuscript 3, and consistent 
with previous research, correlates of high SED and ST include a wide range of factors across biological, behavioural, and environmental domains. ${ }^{5,9}$

Results of this research are also in line with recommendations from the World Health Organization. Recognizing the importance of PA and its relationship with death and disease worldwide, the World Health Organization (WHO) has done a significant amount of work in the field of healthy active living. In May 2004, they adopted the "Global Strategy on Diet, Physical Activity and Health". ${ }^{10}$ This strategy has four main objectives: reduce the risk of chronic disease related to physical inactivity and poor diet through population and public health interventions; to increase awareness and understanding of PA and diet and their influence on health; to develop, strengthen, and implement national policies and action plans on diet and PA; and to monitor science and promote research on PA and diet. ${ }^{10}$ Below, I have positioned the results of this dissertation to align with the objectives outlined by the WHO to suggest areas where we need to focus future research. These recommendations are not presented in order of importance, but represent a collection of multi-sectorial efforts to help children live their healthiest life.

\subsubsection{Continue to monitor and evaluate indicators of healthy active living}

This insight is in line with the WHO Global Recommendations on Physical Activity for Health and their Global Strategy on Diet, Physical Activity and Health. ${ }^{10,11}$ As stated by the WHO, ongoing monitoring and evaluation is necessary to evaluate program success and identify areas of opportunity for future interventions; to ensure interventions, programs, and policies are implemented as they were intended; to 
contribute to ongoing improvements through increased knowledge; to inform new and existing policies and interventions; and to ensure transparency and accountability. ${ }^{12}$ Ongoing monitoring requires the collective effort of researchers, governmental and non-governmental groups, health care providers, teachers, and caregivers and requires funding from many different sources including governmental and non-governmental groups, industry, and charitable organizations.

This dissertation focused on awareness of current PA and SB guidelines, and overall levels of SED and ST in 9-11 year old children. Guideline awareness provides important information on overall effectiveness of knowledge dissemination among the general public. Naturally, it will take some time for the guidelines to make their way in to mainstream educational material. For example, it was only in 2015 that Canadian Physical Activity and Sedentary Behaviour Guidelines were included in the Ontario Health and Physical Education Curriculum. ${ }^{13}$ It is hoped that as resources get updated, awareness (and understanding) of the guidelines will also increase. It is important to continue to monitor progress related to awareness of the guidelines and integrate this into new and innovative ways to disseminate information about the guidelines to health care providers and the general public. For example, social media (e.g., Twitter, Facebook) have become invaluable tools for knowledge dissemination that were not available as little as ten years ago, and now account for a whole sub-area of health research. ${ }^{14}$

With respect to monitoring SED and ST in children, in many study sites, ISCOLE has provided the most up-to-date and robust study of child obesity and lifestyle 
behaviours. International surveys like ISCOLE, as well as nationally representative studies (e.g., the Canadian Health Measures Survey) ensure that we continue to monitor childhood behaviours, growth and development. It also enables researchers to test new hypotheses and employs more advanced analytical techniques. For example, consensus on the terms "sedentary" and "sedentary behaviour" has only come recently, ${ }^{15}$ and even now we don't fully understand the importance of sedentary physiology as it relates to chronic disease. ${ }^{16}$ Without proper measurement of various lifestyle behaviours, it is impossible to properly understand complex relationships and therefore plan effective population health interventions to reduce associated co-morbidities.

\subsubsection{Harmonize data collection strategies around the world}

In recent years, due, in part, to advancements in monitoring technology, health researchers around the world lack a coordinated effort in data collection strategies. This was highlighted in Background Manuscript 2, where we saw very little comparable data from country-to-country. When trying to examine comparable data in ISCOLE countries, it was common to see a variable defined differently (e.g., physical inactivity versus SB), have it analyzed using different cut-points (e.g., body mass index using World Health Organization or Centers for Disease Control and Prevention cut points), and/or using different devices or procedures to measure the same health outcome (e.g., physical activity using accelerometers, pedometers, or self-report questionnaires). There are many strengths and limitations to the procedures that researchers choose to use to define, analyze, and measure their variables of interest, but when examining population health trends and effectiveness of global health interventions, I would argue that it is 
more important to have consistent measurements over time, and space, than to capture large amounts of very specific, but incomparable data.

Again, the WHO has been a leader in this area, including the release of the Global Recommendations on Physical Activity and Health in 2010. ${ }^{11}$ This document aimed to provide a consistent message with other jurisdictions and guidance on how to establish culturally relevant PA messaging around the world. This document was largely informed by the same systematic reviews that informed Canadian physical activity guidelines. ${ }^{17-19}$ The WHO has also established a Global Infobase (https://apps.who.int/infobase/) which is a webpage dedicated to collecting, storing, and displaying information about chronic disease, and associated risk factors worldwide. This includes indicators for non-communicable diseases, international comparisons, information on mortalities, and country specific profiles. This represents an impressive effort for data collection and collaboration worldwide and it would be beneficial to see this expand to include a wider range of behaviours (e.g., SB, sleep) and age groups (e.g., not all information is available for children). This is at least partially dependent on data sharing, availability, and re-analysis of current research databases. With increased advocacy for transparency in all fields of research, many have provided compelling reasons for mandatory open access to all publicly funded research databases. ${ }^{20,21}$ While this may take some time to happen, it may have a positive impact in population health research moving forward.

\subsubsection{Ensure timely updates to guidelines and policies}


Canadian PA guidelines have sought to provide health professionals, researchers, and the public with evidence-informed targets to follow for health benefits, and have the potential to significantly contribute to improvements in overall health across Canada. ${ }^{1,22,23}$ Canadian PA guidelines were first released in 1998-2002 by Health Canada and the Canadian Society for Exercise Physiology (CSEP). Through a rigorous and transparent process, they were updated in 2011-2012 through collaborations between the Public Health Agency of Canada, CSEP, the Healthy Active Living and Obesity Research Group, and ParticipACTION. Current PA and SB guidelines were disseminated through a media launch to raise awareness, followed by promotion through researchers, health care providers, governmental and non-governmental organizations, teachers, and those with an interest in healthy active living. To date, the primary dissemination channel for Canadian guidelines has been through mass media campaigns including print, television and radio campaigns, and websites.

With the new guidelines, there was a recommendation that the guidelines be updated on a regular basis. ${ }^{1,2,24,25}$ It was suggested that this follows a cyclical format to minimize workload (i.e., by avoiding updating all guidelines at the same time) and to ensure all guidelines are updated on a timely basis. ${ }^{1}$ Currently work is being completed to update PA and SB guidelines for children and youth and to create a set of integrated, 24-hour guidelines that include moderate- to vigorous-intensity PA, light intensity activity, SB, and sleep. ${ }^{26}$ These are planned to be released in 2016 and will be the first of their kind for any age group, anywhere in the world. As work is done to update guidelines to reflect the best available evidence, it is also important for policy makers 
and health care providers to be flexible and willing to update their information at the same time. In other words, if new guidelines are released, but their uptake is low, the work to update them may be of little value to the general population. This is especially important to keep in mind as was pointed out in Background Manuscript 1 and Manuscript 1, awareness of current guidelines is low among all sectors of the population.

The results of Manuscript 2 and Manuscript 3 provide some suggestions as to what can be included in new SB guidelines. Specifically, the results of these two studies provide clear evidence that to reduce both SED and ST, children should not have electronics in their bedroom, and families should limit the number of TVs in their home. ${ }^{5}$ Further, the results suggest that to reduce SED and ST, efforts should also be made to increase PA and maintain a healthy weight. Public Health England just released guidelines on workplace sedentary time, suggesting that office workers progress towards accumulating at least 2 hours/day of standing and light activity, with the eventual target of 4 hours/day. ${ }^{27}$ This is another suggestion that may be adapted for the school day, with the introduction of standing workstations in the classroom. Preliminary research in this area has shown that classroom standing desks have been shown to increase energy expenditure (albeit modestly), decrease sitting time, and have no detrimental effects on productivity when compared to seated classrooms. ${ }^{28,29}$

Good news is that many children accumulate on average almost 60 minutes of physical activity per day. ${ }^{30}$ This is not quite the same as accumulating 60 minutes of activity each day of the week, but this is at least a first step in the right direction. Also, 
although most children are exceeding current ST guidelines, they are exceeding them by approximately 35 minutes. ${ }^{5}$ So although there is still a lot of work to do, we may not need to change behaviours from one end of the spectrum to the other, and work to nudge people across the scale. ${ }^{31}$

\subsubsection{Strive for continual improvement of physical activity and sedentary behaviour guidelines and messaging}

Current Canadian PA and SB guidelines were developed based on a rigorous and transparent process and informed by the best available evidence. ${ }^{1}$ The SB guidelines were the first evidence informed guidelines in the world. The background work to inform the guidelines and the process papers to introduce the guidelines are heavily

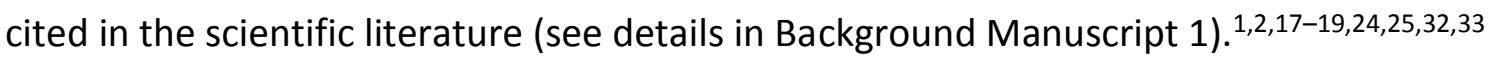
However, few people can correctly identify what the guidelines actually are. Manuscript 1 showed that children are more likely to correctly identify PA guidelines (compared to reports from parents, pediatricians, and the general population) but awareness of the ST guidelines is low. This raises two questions. First, what have we been doing wrong when trying to promote the guidelines among the general public, and second, does it actually matter if people can correctly cite the guidelines.

Manuscript 1 showed that levels of PA or SB did not differ between those who could correctly identify, and those who could not correctly identify current guidelines. Children also seemed to have fairly accurate self-perceptions of their activity status. This was especially true when looking at MVPA and ST, where children could correctly selfidentify as being more, or less active than their peers. This suggests that it does not 
matter (at least in children), if they actually know the targets they need to meet in order to meet them. To my knowledge, this was the first paper that looked at children's knowledge of current guidelines; however, the results of this study are limited in that it was based on a small, cross-sectional sample, of children in the Ottawa area, and ST was measured using self-report questionnaires. Future work should delve deeper in this issue to gain a better understanding of the importance of knowledge and understanding of guidelines. It would be especially interesting to measure both children and parental awareness of the guidelines to determine who is the more important audience for public health messages. It is possible that at least in children, parental knowledge of the guidelines is most important in predicting activity status. We could not find any studies that examined the relationship between adult's knowledge or awareness of guidelines and activity status and so we could not borrow from this literature to better our understanding of the subject. It is possible that as long as children are aware that increased PA and decreased SB (especially ST) is associated with good health, then the specifics of the guidelines do not matter.

Determining how dissemination of the guidelines can be improved is beyond the scope of this discussion. However, this does not diminish the importance of the question. Future guidelines may want to consider more specific recommendations. For example, within the PA guidelines, there are very specific and measurable recommendations (e.g., vigorous-intensity PA at least 3 days per week). ${ }^{1}$ This makes it possible for researchers to examine benchmarks in PA levels and then confirm (or disprove) their association with improved health. SB guidelines provide an overarching 
recommendation to reduce SB, with caveats to limit ST to no more than two hours per day and limit passive transport. ${ }^{2}$ It is possible to measure general reductions in SED, but practically, the ST guidelines are used most often operationally to measure guideline adherence. With the proliferation of research in this area, and the widespread use of accelerometers, there is now a wealth of data that can possibly inform more specific recommendations.

As discussed in Background Manuscript $1,{ }^{3}$ it is also possible that low levels of awareness of the SB guidelines may be in part due to the terminology used. Although people may be familiar with the concept of ST (i.e., television, video game, or computer use), they may not be familiar with the concept of SB as a distinct health risk factor. ${ }^{34}$ Furthermore, influencing decisions to adopt a new behaviour (e.g., reduce SB) may require different promotion and messaging strategies than influencing the decision to discard an old innovation (e.g., continue to follow the old PA guides). With the proliferation of SB research, it is possible that knowledge of the guidelines is set to increase in the near future. A systematic review led by the Centers for Disease Control Task Force showed insufficient evidence to determine effectiveness of stand-alone mass media campaigns for increasing PA. ${ }^{35}$ For successful knowledge dissemination, they suggest that media campaigns be integrated into a broader, multicomponent, community-wide intervention. ${ }^{35}$

\subsubsection{Refine methods for measuring sedentary behaviour in children and youth}

Previous work has shown that ST is often used as a proxy measure for SED. ${ }^{36}$ ST accounts for only about a third of SED, ${ }^{36}$ with the rest of the SED being spent in a variety 
of other pursuits such as reading, passive transport, or eating. With ST accounting for only a small proportion of SED, the appropriateness of using ST as a proxy measure for SED has been questioned. ${ }^{36}$ Still, many studies use measures of SED and ST interchangeably, and make public health messages based on these mixed results. This is important since increased ST is consistently associated with poor health in children and youth, but the relationship between increased SED and increased disease risk is less clear. ${ }^{37,38}$ We also saw in Manuscript 1 that ST, but not SED was associated with selfperceptions of physical activity. Background Manuscript 2 showed that consistent with previous work, boys engaged in more ST, and are less likely to meet ST guidelines than girls ${ }^{9,39-41}$ but girls accumulated more SED than boys. ${ }^{9,42}$ In ISCOLE, participants from the China site engaged in the highest amount of SED ( 9.4 hours per day), but had the second lowest ST score (approximately 1.9 hours per day).

Although activity monitors (e.g., accelerometers, pedometers, or inclinometers) are invaluable for assessing movement behaviours, they provide little information about the type of activity that is being performed. For example, they are unable to distinguish between sitting watching TV and sitting reading a book. This may cause issues with data interpretation and recommendations and suggests to researchers that self-report measures may be more informative. However, previous work has shown that self-report measures do not necessarily provide an accurate depiction of habitual activity. In fact, self-report measures of PA are generally over-reported in children and in adults. ${ }^{43,44}$ This indicates that researchers should use a combination of self-report and directly measured activity. But again, the answer is not that simple, as it is important to 
minimize burden on participants. A carefully chosen, and limited number of questions paired with an unobtrusive activity monitor (e.g., waist or wrist worn), worn for a minimal time period (e.g., no more than 7 days), may be acceptable to most participants. Recent work has shown acceptable reliability with as few as four days of monitoring for sitting time, but seven days of monitoring time for MVPA. Even with acceptable reliability, it is likely that day-to-day variations in sitting time will be high (as much as 4.5 hours/day). ${ }^{45}$

\subsection{Summary of strengths and limitations of this work}

There are many strengths and limitations of this work; some limited to specific sections included within the dissertation, and some related to the thesis as a whole. A detailed discussion of study-specific strengths and limitations is presented in each manuscript. Here I aim to discuss the broader strengths and limitations of the thesis as a whole, and how they related to the larger research literature.

\subsubsection{Strengths}

By far the most significant strength of this dissertation is the collaboration that went into every aspect of the work. Each manuscript benefited from collaborating with some of the best pediatric health researchers in the world. Beyond the improvements these collaborations made to the quality of the work presented in this dissertation, they also provided opportunity for me to improve as a researcher. These collaborations provided countless learning opportunities for myself, and provided me with a great deal of exposure to research (beyond just the manuscripts presented within this dissertation). This also ensured that my analytical framework was sound, and that the 
work was disseminated widely. For example, Manuscript 2 has already been deemed "highly accessed" by the International Journal of Behavioural Nutrition and Physical Activity. Finally, working with many collaborators, from many different research groups from around the world, provided me with exposure to work with various types of work styles, beliefs and habits. I believe that this last point has made me a more flexible researcher and will benefit me when trying to develop my own research program and collaborate with others in the future.

Background Manuscript 1 and Manuscript 1 provided a comprehensive report on awareness of PA and SB guidelines in Canada. This had not been examined since the new guidelines were released in 2011-2012, and an important contribution to public health messaging associated with updates to the guidelines moving forward.

Background Manuscript 2, Manuscript 2, and Manuscript 3 made use of the ISCOLE dataset. As highlighted in Background Manuscript 2, ISCOLE provides the most up-todate and most robust data on obesity and PA in 9-11 year old children around the world. ${ }^{4}$ With ISCOLE, Manuscript 3 is able to provide a comprehensive look at SED and ST in countries around the world, including some countries that have never published information in this area. This work was also the first to analyse correlates of SED and ST in such a large, geographically diverse study sample, using sophisticated analytical techniques. The multilevel modelling analytical framework that was used for data analysis in Manuscripts 1, 2, and 3 has ensured that they will provide a significant contribution to the field. Multilevel modelling was used to properly account for the hierarchical nature of the data collected in ISCOLE. Manuscript 2 was designed to test 
the relationships between SED and ST in children around the world. Multilevel models require relatively advanced statistical techniques, but provide a robust examination of various behaviours while accounting for differences across study sites. ${ }^{6,46}$

\subsubsection{Limitations}

The biggest limitation associated with this work is the generalizability of the findings. ISCOLE participants were primarily recruited through convenience samples, thus the within-site samples were not designed to be nationally representative. ${ }^{4}$ To understand how to better interpret results from ISCOLE, and to make informed recommendations, Background Manuscript 2 aimed to determine if ISCOLE participants were comparable to their peers. Unfortunately, due to limitations in data availability, we recommend that results from ISCOLE should be used with appropriate caution when planning country-level population health interventions.

ISCOLE data are also limited to children aged 9-11 years of age, with little work done to compare results between boys and girls. The results should be used with caution when generalizing to other age groups, or when developing messaging specifically for boys or girls. We did run all models for the total sample, and then separately for boys and girls, however, we did calculate any interactions between boys and girls and therefore cannot report on the between group significance. Another major limitation associated with this work is that it is entirely based on cross-sectional datasets and therefore we cannot comment on the direction of causation. This also opens up the possibility that the results shown here were found by chance and are not true (i.e., Type 1 error), or that we missed associations that are a true reflection of the truth (i.e., Type 
2 error). The likelihood of making these errors was minimized by the statistical techniques used throughout this work, ${ }^{46}$ and through comparisons with previous work; however, they must be considered when interpreting the results.

Manuscript 1 used data from CAPL, and from the ISCOLE-CAPL subset. The questions related to guideline awareness were captured via child-report questionnaire as part of CAPL, and we had no measure of parental awareness of the guidelines. Given the disconnect between the high level of guideline awareness reported in Manuscript 1, and the low level of awareness reported in parents, and healthcare providers in Background Manuscript 2, it would have been interesting to have both child- and parent-report data from the same population. Especially given that children are not autonomous in the majority of their choices, and rely largely on parental access to activities. Future work should compare the relationship between guideline awareness and activity status among children, parents, and teachers. This could help identify who to target for guideline-related public health messages.

Further limitations of this work are related to the type of analysis performed. This work was limited to quantitative analysis and it is possible it could have benefited from mixed-methods research. However, logistically, qualitative research can be difficult with large sample sizes, especially over a large geographical area. But, for example, when examining knowledge and awareness of PA and SB guidelines, it may have been worthwhile to conduct focus groups or interviews to gain a better understanding on how people used, or did not use the guidelines. This may be an area for future research. 


\subsection{Knowledge translation strategy}

The primary method for disseminating the results of this work was through peer-reviewed publications and conference presentations, with additional outreach through social media outlets (Healthy Active Living and Obesity Research website: www.haloresearch.ca, and twitter: @AllanaLeBlanc, @HALOresearch).When possible, manuscripts were, and will be published in open access journals to ensure maximum reach. Conferences were targeted with maximum reach in mind with assistance from travel grants from the Faculty of Graduate and Postdoctoral Studies at the University of Ottawa, Population Health at the University of Ottawa, the Canadian Institutes for Health Research, Mitacs, and the Healthy Active Living and Obesity Research Group at the Children's Hospital of Eastern Ontario Research Institute. Presentations associated with this thesis, and on related projects that were completed during my doctoral training, were given at the Canadian Society for Exercise Physiology Annual Meeting, the Canadian Obesity Summit, the Global Summit on the Physical Activity of Children, the International Society for Behavioural Nutrition and Physical Activity Conference, The Obesity Society Annual Meeting, and the World Congress on Sleep Medicine. The wide breadth of conferences I was able to attend (both geographically and content-wise) allowed me invaluable networking opportunities with leaders in the field of healthy active living. Since the majority of the manuscripts included in this dissertation involved collaborating with researchers from around the world, these conferences also provided me time to meet face-to-face with many of my co-authors. 


\subsection{Conclusions}

This dissertation provides a comprehensive look at awareness of public health guidelines, and SB in children. Specifically, it aimed to examine awareness of current PA and ST guidelines and the association with habitual activity in Canadian children, identify correlates of SED and ST in a Canadian sample of 9-11 year-old children, and identify correlates of SED and ST in 9-11 year-old children from 12 countries around the world. We showed that among children, awareness of PA guidelines is high, but low for ST guidelines, and that their awareness was not associated with habitual activity status. We also identified correlates of SED and ST in Canada, and around the world. We identified some correlates that may be easily modified through public health messaging, and others that may require more in depth behavioural interventions. Overall, several recommendations were made for future work including to continue to monitor and evaluate indicators of healthy active living; to harmonize data collection strategies around the world; to strive for continual improvement of physical activity and sedentary behaviour guidelines and messaging; and to refine methods for measuring sedentary behaviour in children and youth. 


\section{References}

1. Tremblay, M. S. et al. New Canadian Physical Activity Guidelines. Appl. Physiol. Nutr. Metab. 36, 36-46 (2011).

2. Tremblay, M. S. et al. Canadian Sedentary Behaviour Guidelines for Children and Youth. Appl. Physiol. Nutr. Metab. 36, 59-64 (2011).

3. LeBlanc, A. G. et al. Knowledge and awareness of Canadian Physical Activity and Sedentary Behaviour Guidelines: a synthesis of existing evidence. Appl. Physiol. Nutr. Metab. 40, 1-9 (2015).

4. Katzmarzyk, P. T. et al. The International Study of Childhood Obesity, Lifestyle and the Environment (ISCOLE): design and methods. BMC Public Health 13, 900 (2013). 5. LeBlanc, A. G. et al. Correlates of objectively measured sedentary time and selfreported screen time in Canadian children. Int. J. Behav. Nutr. Phys. Act. 12, 38 (2015). 6. Bell, B. B., Ene, M., Smiley, W. \& Schoeneberger, J. A. A multilevel model primer using SAS PROC MIXED. (University of South Carolina, 2013).

7. Sallis, J. F., Owen, N. \& Fisher, E. B. in Health behavior and health education: Theory, research, and practice (4th ed.) (eds. Glanz, K., Rimer, B. K. \& Viswanath, K.) 465-485 (Jossey-Bass, 2008).

8. Owen, N. et al. Adults' Sedentary Behavior: Determinants and Interventions. Am. J. Prev. Med. 41, 189-196 (2011).

9. Temmel, C. S. D. \& Rhodes, R. Correlates of Sedentary Behaviour in Children and Adolescents Aged 7-18: A Systematic Review. Health Fit. J. Can. 6, 119-199 (2013). 
10. World Health Organization. Global strategy on diet, physical activity and health. (2004).

11. World Health Organization. Global recommendations on physical activity for health. (2010). at

<http://www.who.int/dietphysicalactivity/publications/9789241599979/en/index.html>

12. World Health Organization. A framework to monitor and evaluate implementation: WHO strategy on diet, physical activity and health. (2008).

13. Ministry of Education. Health and physical education: the Ontario curriculum grades 1-8. (Ontario Public Service, 2015). at <www.edu.gov.on.ca>

14. Lefebvre, R. C. \& Bornkessel, A. S. Digital Social Networks and Health. Circulation 127, 1829-1836 (2013).

15. Sedentary Behaviour Research Network. Letter to the Editor: Standardized use of the terms 'sedentary' and 'sedentary behaviours'. Appl. Physiol. Nutr. Metab. 37, 540542 (2012).

16. Lynch, B. M. \& Owen, N. Too Much Sitting and Chronic Disease Risk: Steps to Move the Science Forward. Ann. Intern. Med. 162, 146-147 (2015).

17. Janssen, I. \& LeBlanc, A. G. Systematic review of the health benefits of physical activity and fitness in school-aged children and youth. Int. J. Behav. Nutr. Phys. Act.7, 40 (2010).

18. Warburton, D. E., Charlesworth, S., Ivey, A., Nettlefold, L. \& Bredin, S. S. A systematic review of the evidence for Canada's Physical Activity Guidelines for Adults. Int. J. Behav. Nutr. Phys. Act. 7, 39 (2010). 
19. Paterson, D. \& Warburton, D. Physical Activity and Functional Limitations in Older Adults: A Systematic Review Related to Canada's Physical Activity Guidelines. Int. J. Behav. Nutr. Phys. Act. 7, (2010).

20. Krumholz HM \& Peterson ED. Open access to clinical trials data. JAMA 312, 1002-1003 (2014).

21. Spertus, J. A. The Double-Edged Sword of Open Access to Research Data. Circ. Cardiovasc. Qual. Outcomes 5, 143-144 (2012).

22. Grimshaw, J. \& Russell, I. Achieving health gain through clinical guidelines. I: Developing scientifically valid guidelines. Qual. Health Care 2, 243-248 (1993).

23. Tremblay, M. S., Shephard, R. J. \& Brawley, L. R. Research that informs Canada's physical activity guides: an introduction. Appl. Physiol. Nutr. Metab. 32, S1-S8 (2007). 24. Tremblay, M. S. et al. Canadian Physical Activity Guidelines for the Early Years (aged 0-4 years). Appl. Physiol. Nutr. Metab. 37, 345-356 (2012).

25. Tremblay, M. S. et al. Canadian Sedentary Behaviour Guidelines for the Early Years (aged 0-4 years). Appl. Physiol. Nutr. Metab. Physiol. Appliquée Nutr. Métabolisme 37, 370-391 (2012).

26. Chaput, J., Carson, V., Gray, C. E. \& Tremblay, M. S. Importance of All Movement Behaviors in a 24 Hour Period for Overall Health. Int. J. Environ. Res. Public. Health 11, 12575-12581 (2014).

27. Buckley, J. P. et al. The sedentary office: a growing case for change towards better health and productivity. Expert statement commissioned by Public Health 
England and the Active Working Community Interest Company. Br. J. Sports Med. bjsports-2015-094618 (2015). doi:10.1136/bjsports-2015-094618

28. Benden, M. E., Zhao, H., Jeffrey, C. E., Wendel, M. L. \& Blake, J. J. The Evaluation of the Impact of a Stand-Biased Desk on Energy Expenditure and Physical Activity for Elementary School Students. Int. J. Environ. Res. Public. Health 11, 9361-9375 (2014).

29. Torbeyns, T., Bailey, S., Bos, I. \& Meeusen, R. Active Workstations to Fight Sedentary Behaviour. Sports Med. 44, 1261-1273 (2014).

30. Colley, R. C. et al. Physical activity of Canadian children and youth: Accelerometer results from the 2007 to 2009 Canadian Health Measures Survey. Health Rep. 22, 15-23 (2011).

31. Loewenstein, G., Asch, D. A., Friedman, J. Y., Melichar, L. A. \& Volpp, K. G. Can behavioural economics make us healthier? BMJ 344, e3482 (2012).

32. Tremblay, M. S. et al. Systematic review of sedentary behaviour and health indicators in school-aged children and youth. Int. J. Behav. Nutr. Phys. Act. 8, 98 (2011).

33. Timmons, B. W. et al. Systematic review of physical activity and health in the early years (aged 0-4 years). Appl. Physiol. Nutr. Metab. 37, 773-792 (2012).

34. Tremblay, M. S., Colley, R. C., Saunders, T. J., Healy, G. N. \& Owen, N. Physiological and health implications of a sedentary lifestyle. Appl. Physiol. Nutr. Metab. 35, 725-740 (2010).

35. Brown, D. R. et al. Stand-Alone Mass Media Campaigns to Increase Physical Activity: A Community Guide Updated Review. Am. J. Prev. Med. 43, 551-561 (2012). 
36. Biddle, S. J. H., Gorely, T. \& Marshall, S. J. Is Television Viewing a Suitable Marker of Sedentary Behavior in Young People? Ann. Behav. Med. 38, 147-153 (2009).

37. Saunders, T. J. et al. Prolonged sitting and markers of cardiometabolic disease risk in children and youth: a randomized crossover study. Metabolism. 62, 1423-1428 (2013).

38. Colley, R. C. et al. The association between accelerometer-measured patterns of sedentary time and health risk in children and youth: results from the Canadian Health Measures Survey. BMC Public Health 13, 200 (2013).

39. Cao, H. et al. Screen time, physical activity and mental health among urban adolescents in China. Prev. Med. 53, 316-320 (2011).

40. Cui, Z., Hardy, L. L., Dibley, M. J. \& Bauman, A. Temporal trends and recent correlates in sedentary behaviours in Chinese children. Int. J. Behav. Nutr. Phys. Act. 8, $1-8$ (2011).

41. Anderson, S. E., Economos, C. D. \& Must, A. Active play and screen time in US children aged 4 to 11 years in relation to sociodemographic and weight status characteristics: a nationally representative cross-sectional analysis. BMC Public Health 8, 366 (2008).

42. Rodrigues, A. M. M. et al. Confounding effect of biologic maturation on sex differences in physical activity and sedentary behavior in adolescents. Pediatr. Exerc. Sci. 22, 442-453 (2010). 
43. Adamo, K. B., Prince, S. A., Tricco, A. C., Connor-Gorber, S. \& Tremblay, M. A comparison of indirect versus direct measures for assessing physical activity in the pediatric population: A systematic review. Int. J. Pediatr. Obes. 4, 2-27 (2009).

44. Prince, S. A. et al. A comparison of direct versus self-report measures for assessing physical activity in adults: a systematic review. Int. J. Behav. Nutr. Phys. Act. 5, 56 (2008).

45. Barreira, T. V. et al. Intra-individual and inter-individual variability in daily sitting time and MVPA. J. Sci. Med. Sport in press (2015)

46. Diez-Roux, A. V. Multilevel Analysis in Public Health Research. Annu. Rev. Public Health 21, 171-192 (2000). 


\section{Appendix A \\ Background Manuscript 1}

Are participant characteristics from ISCOLE study sites comparable to the rest of their country?

This manuscript has been written and formatted to adhere to the specifications for submission to a special issue of the International Journal of Obesity. This manuscript is currently under review. 


\title{
Are participant characteristics from ISCOLE study sites comparable to the rest of their country? \\ Short title: Generalizability of ISCOLE
}

\begin{abstract}
Allana G. LeBlanc ${ }^{1,2^{*}}$, Peter T. Katzmarzyk ${ }^{3}$, Tiago V. Barreira ${ }^{3,4}$, Stephanie T. Broyles ${ }^{3}$, Jean-Philippe Chaput ${ }^{1,2}$, Timothy S. Church ${ }^{3}$, Mikael Fogelholm ${ }^{5}$, Deirdre M. Harrington ${ }^{6}$, Gang $\mathrm{Hu}^{3}$, Rebecca Kuriyan ${ }^{7}$, Anura Kurpad ${ }^{7}$, Estelle V. Lambert ${ }^{8}$, Carol Maher ${ }^{9}$, José Maia $^{10}$, Victor Matsudo ${ }^{11}$, Timothy Olds ${ }^{9}$, Vincent Onywera ${ }^{12}$, Olga L. Sarmiento ${ }^{13}$, Martyn Standage ${ }^{14}$, Catrine Tudor-Locke ${ }^{3}$, Pei Zhao ${ }^{15}$ and Mark S. Tremblay ${ }^{1}$, for the ISCOLE Research Group

${ }^{1}$ Children's Hospital of Eastern Ontario Research Institute, Ottawa, Canada; ${ }^{2}$ University of Ottawa, Ottawa, Canada; ${ }^{3}$ Pennington Biomedical Research Center, Baton Rouge, United States; ${ }^{4}$ University of Syracuse, Syracuse, United States; ${ }^{5}$ University of Helsinki, Helsinki, Finland; ${ }^{6}$ University of Leicester, Leicester, United Kingdom; ${ }^{7}$ St. Johns Research Institute, Bangalore, India; ${ }^{8}$ University of Cape Town, Cape Town, South Africa; ${ }^{9}$ School of Health Sciences/Sansom Institute, University of South Australia, Adelaide, Australia; ${ }^{10}$ Faculdade de Desporto, University of Porto, Porto, Portugal; ${ }^{11}$ Centro de Estudos do Laboratório de Aptidão Física de São Caetano do Sul (CELAFISCS), Sao Paulo, Brazil;

${ }^{12}$ Kenyatta University, Nairobi, Kenya; ${ }^{13}$ School of Medicine, Universidad de los Andes, Bogota, Colombia ${ }^{14}$ University of Bath, Bath, United Kingdom; ${ }^{15}$ Tianjin Women's and Children's Health Center, Tianjin, China
\end{abstract}

*Correspondence to:

Allana G. LeBlanc

Healthy Active Living and Obesity Research Group

Children's Hospital of Eastern Ontario Research Institute

401 Smyth Road

Ottawa, Ontario, Canada

K1H 8L1

Phone: +1-613-737-7600 ext.4191

Fax: +1-613-738-4800

E-mail: alleblanc@cheo.on.ca

Conflict of interest: ISCOLE was funded by The Coca-Cola Company. The funder had no role in the design and conduct of the study; collection, management, analysis and interpretation of the data; and preparation, review or approval of the manuscript. The authors declare no other conflicts of interest. 


\section{Abstract}

Background/objectives: The International Study of Childhood Obesity, Lifestyle and the Environment (ISCOLE) provides robust, multi-national information on physical activity, diet and weight status in 9-11 year-old children around the world. The purpose of this analysis was to examine the similarities and differences between participant characteristics from ISCOLE sites and data from nationally representative surveys from ISCOLE countries (Australia, Brazil, Canada, China, Colombia, Finland, Kenya, India, Portugal, South Africa, the United Kingdom, and the United States). Methods:

Distributions of characteristics were assessed within each ISCOLE country-level database, and compared to published data from national or regional surveys, where available. Variables of comparison were identified a priori and included body mass index (BMI), physical activity (accelerometer-determined steps per day), and screen time (child-report). Results: Of twelve countries, data on weight status (BMI) were available in eight countries, data on measured physical activity (steps per day) were available in five countries, and data on self-reported screen time were available in nine countries. The five ISCOLE countries that were part of the Health Behaviour in School-aged Children Survey (i.e., Canada, Finland, Portugal, United Kingdom (England), and United States) also provided comparable data on self-reported physical activity. Available country-specific data often used different measurement tools or cut-points, making direct comparisons difficult. Where possible, ISCOLE data were re-analysed to match country-level data, but this step limited between-country comparisons. Conclusions: From the analyses performed, the ISCOLE data do not seem to be systematically biased; 
however, due to limitations in data availability, data from ISCOLE should be used with appropriate caution when planning country-level population health interventions. This work highlights the need for harmonized measurement tools around the world while accounting for culturally specific characteristics, and the need for collaboration across study centres and research groups. 


\section{Introduction}

The prevalence of paediatric obesity and related lifestyle behaviors has been examined in many countries; however, the International Study of Childhood Obesity, Lifestyle and the Environment (ISCOLE) represents the most up-to-date, directly measured, harmonized study of several countries ranging widely in level of human development. ${ }^{1}$ Previous multi-national studies have focused on one geographic region (e.g., HELENA in Europe [Healthy Lifestyle in Europe by Nutrition in Adolescents] ${ }^{2}$ ), or limited their inclusion to developed, or high income countries (e.g., HBSC [Health Behaviour in School-aged Children $]^{3,4}$ ). ISCOLE aimed to recruit study sites with diverse geographical distribution from around the world, and drawn from low-, middle-, and high-income countries. Further, many previous studies have been limited by small sample sizes, indirect measurements, and/or the inability to generalize their findings to larger populations. With so many small, isolated, and non-representative studies, it becomes difficult to inform population health interventions, especially on a global scale.

ISCOLE participants were primarily recruited through convenience samples, thus the within-site samples were not designed to be nationally representative. However, to understand how to better interpret results from ISCOLE, and to make informed recommendations, it is important to understand whether ISCOLE participants are comparable to their country-level peers as a whole, or if they represent a unique subset of the larger population. The purpose of this paper was to determine if data collected at ISCOLE study sites were representative of their site country. This work may be used to better understand the limitations, potential biases and generalizability of ISCOLE results, 
and to understand current gaps in health and obesity-related knowledge in participating countries.

\section{Methods}

\section{International Study of Childhood Obesity, Lifestyle and the Environment}

Details on participant recruitment and sampling strategy have been reported in the ISCOLE methods paper. ${ }^{1}$ In brief, data collection occurred from September 2011 through to December 2013 with a goal of recruiting at least 500 participants, aged 9-11 years, from each study site. Sites made an effort to stratify their sample by indicators of socio-economic status to maximize variability, and generally had a goal of including at least 20 schools, with approximately $25-30$ children per school. ${ }^{1}$ Many countries included both private and public schools, although all sites limited their data collection to large cities, and urban or suburban schools (i.e., no country collected data from participants living in remote or rural areas, or attending rural schools). To ensure that ISCOLE participants represented diverse backgrounds and circumstances, study sites were chosen from diverse geographic regions around the world (i.e., Europe, Africa, the Americas, South Asia, and the Western Pacific) and across different levels of socioeconomic indicators (i.e., World Bank classification, Human Development Index, and the Gini Index). The ISCOLE coordinating center, located at the Pennington Biomedical Research Center in Baton Rouge, Louisiana, was responsible for overall administration of the study. This project was approved by the relevant research ethics boards at Pennington Biomedical Research Center, at each ISCOLE study site, and at the respective 
school boards. Written informed parental consent and child assent were obtained for all participants as required.

ISCOLE variables used for the present analysis were limited to those common in national surveys and surveillance systems, including body mass index (BMI), household income, physical activity and screen time. Anthropometric variables (height, weight, $\mathrm{BMI}$ ) were collected and calculated following standard procedures and measurement tools. ${ }^{1}$ BMI was calculated and weight status was determined using various cut-points (e.g., World Health Organization, Center for Disease Control and Prevention) to maximize comparability with nationally representative data. Accelerometer derived variables (light-, moderate- and vigorous-intensity physical activity, and step counts) were obtained via 24-hour wear protocol using the waist-worn ActiGraph GT3X+ triaxial accelerometer (ActiGraph LLC, Pensacola, FL, USA) and validated data reduction strategies. ${ }^{5,6,7}$ Step counts were calculated using the manufacturer's default filter. Data on behavioral characteristics (e.g., self-reported physical activity, and screen time) were obtained via a child-report questionnaire. For the purpose of this work, physical activity guidelines were defined as $\geq 60$ minutes of daily moderate- to vigorous-intensity physical activity and sedentary behavior guidelines were defined as $\leq 2$ hours of self-reported recreational screen time per day. These guidelines are consistent with those from many countries, including Australia, ${ }^{10}$ Canada, ${ }^{11,12}$ the U.K., ${ }^{13}$ the U.S., ${ }^{14}$ and the World Health Organization. ${ }^{15}$ Socio-economic status was measured via parent-reported household income. Further details on all measurement procedures and questionnaires used in ISCOLE can be found elsewhere. ${ }^{1}$ 


\section{Nationally representative data}

To gain access to nationally representative data, and to understand the intricacies of the datasets, the primary investigator from each ISCOLE study site was asked to provide information for their respective country. As the primary investigators for ISCOLE were chosen based on their expertise in paediatric obesity research, it was believed that they would be aware of relevant studies and data sources. They were asked to use their best judgement when identifying information; however, some of the available nationally representative data may be considered to be out of date. The writing group for this paper agreed that it was more important to have comparable and well-collected data (e.g., similar age group, directly measured variables) than to necessarily have the most recent data. See Additional File 2 for summaries of included studies.

When no data were available, cells were left blank. It is important to note that all variables included in this paper were identified a priori and deemed the most relevant to the ISCOLE project and most likely to be captured in other studies. Therefore, some countries have a greater number of areas with no data than other countries, and this may highlight a paucity of robust data in certain areas.

\section{Results}

ISCOLE data were available for 9-11-year-old children from Australia $(n=516)$, Brazil $(n=541)$, Canada $(n=541)$, China $(n=537)$, Colombia $(n=905)$, Finland $(n=525)$, India ( $n=584)$, Kenya $(n=563)$, Portugal $(n=724)$, South Africa $(n=513)$, the U.K. $(n=525)$, and the U.S. $(n=554)$. Characteristics of ISCOLE countries can be found in Table 1 , and 
characteristics of participants from each ISCOLE site can be found in Table 2 . The proportion of children overweight or obese, accelerometer-determined steps per day, and the proportion of children exceeding current screen time guidelines are presented in Figures 1-3, respectively. Figure 4 shows the proportion of children that self-reported meeting physical activity guidelines (i.e. 60 minutes of moderate- to vigorous-intensity physical activity per day) in ISCOLE, and in the HBSC.

\section{Europe (Finland, Portugal, United Kingdom)}

All of the ISCOLE site countries in Europe were classified as high-income countries. They were also all part of the HBSC survey. ${ }^{4}$ Based on the results from HBSC, Finland provided self-reported data showing that $38 \%$ of 11 year old boys, and $25 \%$ of 11 year old girls met physical activity guidelines, ${ }^{4}$ which is higher than $22 \%$ of $9-11$ year old boys and $10 \%$ of $9-11$ year old girls who self-reported that they met physical activity guidelines in ISCOLE. Additional data from Tammelin et al., and the Foundation for Sport and Health Science, found that Finnish children (aged 9-10 years) accumulated 10,506 steps per day, compared to 10,485 steps per from ISCOLE participants. Data looking at screen time came from the 2010 HBSC, which reported that $61 \%$ of 11 year-old boys, and $58 \%$ of 11 year-old girls self-reported that they exceeded screen time recommendations on weekdays. This was lower than in ISCOLE, which showed that $84 \%$ of boys, and $74 \%$ of girls exceeded the recommendation. We were unable to find any comparable data reporting the proportion of Finnish children considered overweight or obese. Therefore, data from ISCOLE Finland are consistent with previous data for 
directly measured physical activity (but lower for self-reported physical activity), provide slightly higher estimates for screen time, and inconclusive with respect to weight status. Portuguese children who were part of the HBSC study self-reported higher physical activity levels ( $23 \%$ of 11 year-old boys, and $14 \%$ of 11 year old girls are meeting physical activity guidelines) than self-report data from ISCOLE (9\% of boys and $4 \%$ of girls). ${ }^{4}$ Reports from HBSC suggest 11 year-old children are watching more TV ( $61 \%$ of boys and $60 \%$ of girls exceed screen time guidelines) compared to ISCOLE participants (55.3\% of boys and $43.0 \%$ of girls exceed screen time guidelines). Regarding weight status, Portugal was able to provide comparable data 10 year-old children from the Plataforma Contra a Obesidade. ${ }^{18}$ This showed similar proportions of children considered overweight or obese for boys ( $50.0 \%$ compared to $51.4 \%$ in ISCOLE) and girls (45.3 versus $42.3 \%$ in ISCOLE). Overall, participants from ISCOLE Portugal reported lower levels of physical activity, but similar levels of screen time, and a similar proportion of children were considered overweight or obese compared to other surveys.

Because the ISCOLE site in the U.K. was based in England, we aimed to obtain comparable data from England, rather than across the U.K. wherever possible. Data from England came primarily from the Millennium Cohort Study (MCS), with additional data coming from HBSC. According to the MCS (children aged 7-8 years), boys averaged 10,739 steps per day and girls averaged 9,699 steps per day. ${ }^{19}$ This was very similar to what was seen in ISCOLE with boys averaging 10,675 steps per day and girls averaging 9,435 steps per day. Self-reported data from HBSC showed that $33 \%$ of 11 year-old boys and $20 \%$ of 11 year-old girls met physical activity guidelines, ${ }^{4}$ whereas in ISCOLE, only 
$19 \%$ of boys and $11 \%$ of girls self-reported that they met the guidelines. With respect to sedentary behavior, results from the HBSC showed that $64 \%$ of 11 year-old boys, and $60 \%$ of 11 year-old girls exceeded screen time guidelines on week days $;{ }^{4}$ results from ISCOLE show that $73 \%$ of boys, and $61 \%$ of girls exceeded the guidelines. Compared to data from 10-11 year-old children from the National Child Measurement Programme, there was a lower proportion of children considered overweight or obese in ISCOLE (33.3\% versus $21.7 \%$ respectively). ${ }^{20}$ Data from ISCOLE seem to be comparable with respect to physical activity levels, but with higher levels of screen time. However, it can be assumed that there are variations in the socio-cultural environment across the rest of the U.K and it is recommended that generalizations to a wider population are made only with appropriate caution.

Africa (Kenya, South Africa)

Kenya was the only study included in ISCOLE considered to be a low-income country; South Africa is considered to be an upper-middle income country. Neither Kenya, nor South Africa identified any nationally representative, or large datasets for comparison. Summaries of the best available evidence for each country was presented in their 2014 Report Card on Physical Activity in Children and Youth and included primarily early results from ISCOLE, suggesting future studies are critically needed. ${ }^{21,22}$ Comparisons for ISCOLE Kenya came from two systematic reviews examining obesity and physical activity transitions in Sub-Saharan Africa. ${ }^{23,24}$ One systematic review reported a range of $35-72 \%$ of children meeting physical activity guidelines, ${ }^{23}$ which is higher than the $11.4 \%$ of children who self-reported meeting the guidelines in 
ISCOLE. The only data we could identify that examined screen time was from ISCOLE and showed that $53.6 \%$ of children exceeded screen time guidelines. However, this may be due to the fact that ISCOLE only sampled from urban and suburban areas as previous research on Kenyan children suggests that screen time is much lower in rural areas. ${ }^{25}$ The systematic review examining the overweight and obesity transition included studies that reported proportions of overweight/obesity between $3.2-12.0 \%,{ }^{24}$ which is lower than $16 \%$ found in ISCOLE. Even though there were little comparable data available in Kenya, Nairobi is an urban hub, with a population of over 3 million people, and one could assume that children from urban Nairobi may not be representative of their rural peers.

Although South Africa was not able to provide data from any nationally representative surveys, information from smaller studies, summarized in their Report Card suggest that $50-59 \%$ of children are meeting physical activity guidelines, and $<50 \%$ of children are meeting screen time guidelines. ${ }^{22}$ These values are more promising than what was shown in ISCOLE, which found that $26.4 \%$ of children self-reported meeting physical activity guidelines, and $36.7 \%$ reported meeting screen time guidelines. Data from the 2010 Survey of Time of Use suggest that on average, children aged 10-17 years watched 3 hours of TV per day. ${ }^{26}$ In ISCOLE, children were asked to self-report habitual $T V$, video game, and computer use with the highest possible value being " $\geq 5$ hours per day". Therefore for analysis, self-reported screen time was presented as a score, rather than total hours of screen time since after 5 hours per day, we could not ascertain the participant's actual amount of screen time. Although ISCOLE can only provide an 
approximate value for daily screen time, it did appear to be similar to what was reported in the Time of Use survey with children averaging approximately 3.1 hours of screen time and 2.0 hours of TV time per day. We were not able to find any comparable data with respect to weight status. Therefore, children from ISCOLE South Africa seem to be slightly less active than reported in other studies, but seem to engage in similar amounts of screen time.

The Americas (Brazil, Canada, Colombia, and the United States)

Canada and the U.S. are both considered high-income countries; whereas Colombia and Brazil are both considered upper-middle-income countries. The Americas, especially Canada and the U.S., were able to provide a significant amount of comparable and nationally representative data. Canada and the U.S. are both included in the HBSC, and both have directly measured, nationally representative surveys (CHMS: Canadian Health Measures Survey, ${ }^{27}$ NHANES: National Health and Nutrition Examination Survey (U.S.)).

Brazil provided comparable data for weight status for 10 year-old children from the Brazilian Institute of Geography and Statistics (BIGS), ${ }^{28}$ but was unable to provide comparable data for physical activity, or screen time. BIGS reported that $33.1 \%$ of their children were considered overweight or obese, compared to a much higher $45.7 \%$ of children considered overweight or obese in ISCOLE.

Comparable data for Canada came primarily from the CHMS (with custom analysis for their 2014 Report Card ${ }^{29}$ ) and showed that only 5\% of school-aged children met current Canadian physical activity guidelines. ${ }^{11,30}$ However, directly measured 
physical activity from 6-10 year old children in the CHMS show that ISCOLE children are less active than children from across Canada. In the CHMS boys averaged 13,217 steps per day and girls averaged 11,745 steps per day compared to 9,891 steps per day for boys and 8,591 steps per day for girls. ${ }^{31}$ Self-reported data from HBSC showed $31 \%$ of boys, and $21 \%$ of girls met the physical activity guidelines, ${ }^{4}$ which was similar to data from ISCOLE, with $26 \%$ of boys, and $18 \%$ of girls self-reporting they met guidelines. With respect to screen time, data came from the $2007-2009$ CHMS and showed that $31 \%$ of children aged 5-11 years exceed screen time guidelines. ${ }^{31}$ This was slightly lower than what was seen in ISCOLE Canada, with $54.4 \%$ of boys, and $40.2 \%$ of girls exceeding guidelines. There was a slightly lower proportion of children considered overweight or obese in ISCOLE (21.9\% of boys and $21.7 \%$ of girls), compared to reports from the CHMS (31\% for boys and 26\% for girls). In Canada, ISCOLE participants engaged in similar amounts of self-reported physical activity (but less directly measured physical activity), greater amounts of screen time, and had a lower proportion of children considered overweight or obese.

Comparable data for levels of physical activity in Colombia came from the ENSIN study (Encuesta Nacional de la Situación Nutricional (National Survey of Nutritional Status)), ${ }^{32}$ and showed that $26 \%$ of children self-reported that they met physical activity guidelines, which is higher than what was seen in ISCOLE (12\% meeting guidelines). The ENSIN study also provided comparable data with respect to screen time and weight status. For screen time, ENSIN showed that fewer children $(59.4 \%$ of boys, and $56.3 \%$ of girls) exceeded screen time guidelines than children from ISCOLE (71.6\% of boys, and 
$60.4 \%$ of girls). ${ }^{33}$ ENSIN also reported a lower proportion of children being overweight or obese than ISCOLE (17.5\% in ENSIN compared to $23.3 \%$ in ISCOLE). ${ }^{33}$ Overall, it appears that children in ISCOLE self-report engaging in less physical activity and more screen time, and are more likely to be overweight or obese than other Colombian children.

Comparable data for U.S. physical activity levels came primarily from the 20032004 NHANES (National Health and Nutrition Examination Survey). Accelerometer data showed that $42.0 \%$ of children aged $6-11$ years were meeting physical activity guidelines, $^{34,35}$ and on average, 10 year old boys were accumulating 10,163 steps per day and girls were accumulating 8.906 steps per day. ${ }^{36}$ This was similar to what was seen in ISCOLE, with boys accumulating only 9,261 steps per day, and girls accumulating only 8,078 steps per day. With respect to screen time, data were presented from the 2009-2010 NHANES and showed that $47.8 \%$ of children aged 9-11 years self-reported that they exceeded screen time guidelines. ${ }^{37}$ The proportion of children exceeding guidelines was higher in ISCOLE, and in 11 year-old boys and girls from $\mathrm{HBSC}^{4}$ (Figure 3). In ISCOLE, $68.3 \%$ of boys exceeded screen time guidelines, and $56.2 \%$ of girls exceeded screen time guidelines. Compared to NHANES, we saw a similar proportion of children considered overweight or obese in ISCOLE (NHANES: boys $=33.2 \%$, girls $=35.2 \%$; ISCOLE boys $=32.4 \%$, girls $=35.6 \%)$. In the U.S. sample of ISCOLE, children were less active, and were less likely to meet screen time guidelines, but had similar weight status to children from across the country.

\section{South Asia (India)}


India is the only study site in South Asia, and is considered a lower-middleincome country. We were unable to identify any comparable datasets. Data collected in ISCOLE show that $28.4 \%$ of Indian children are meeting physical activity guidelines, $30.8 \%$ are meeting screen time guidelines, and $33.7 \%$ are considered overweight or obese.

Western Pacific (Australia, China)

Australia is considered a high-income country and China is considered an uppermiddle-income country. Australia provided comparable data via the ANCNPAS (Australian National Children's Nutrition and Physical Activity Survey), ${ }^{38}$ the National Health Surveys, ${ }^{39}$ and a summary of current evidence via their 2014 Report Card on Physical Activity in Children and Youth. ${ }^{40}$ China was able to provide comparable data via the China Health and Nutrition Survey (CHNS).

Comparable data from Australia came primarily from the Australian Bureau of Statistics. These data showed that $20 \%$ of children aged 5-17 years self-reported that they met physical activity guidelines. ${ }^{39}$ This is consistent with self-reported data from ISCOLE. Australia was also able to provide information from the ANCNPAS showing higher values for number of steps per day $(12,230$ in ANCNPAS compared to 10,262 in ISCOLE). ${ }^{38}$ With respect to screen time, data from the Australian Bureau of Statistics showed that $70 \%$ of children aged $5-17$ years old exceeded screen time guidelines. This was higher than what was seen in ISCOLE, with only $56 \%$ of children exceeding the guidelines. Data from ANCNPAS reported $28.4 \%$ of $9-11$ year old children to be overweight or obese, which is similar to $26.7 \%$ of children considered overweight or 
obese in ISCOLE. Overall, participants from ISCOLE accumulated similar amounts of physical activity, had lower screen time, and had more favourable weight status than their Australian peers.

China provided comparable data on physical activity, screen time, and weight status via the CHNS (China Health and Nutrition Survey). ${ }^{41,42}$ For $6-11$ year-olds, the CHNS reported children averaged 60 minutes (boys), and 48 minutes (girls) of physical activity per day, compared to lower levels in ISCOLE (49.5 minutes for boys and 40.5 minutes for girls). Results for screen time showed that $24.9 \%$ of boys and girls exceeded screen time guidelines, compared to $35.1 \%$ in ISCOLE. However, it is interesting to note that these data came from 2004, and in a longitudinal analysis, the proportion of children who reported $>2$ hours of screen time per day rose from $5.8 \%$ in 1997 , to $24.9 \%$ in 2004, suggesting data from present day may be more comparable. With respect to weight status, the proportion of children considered overweight or obese was much higher in ISCOLE than in the CHNS (ISCOLE boys $=50.1 \%$, ISCOLE girls $=30.6 \%$, versus CHNS boys $=16.6 \%$ and HBSC boys $=10.9 \%)$. ISCOLE China participants accumulated slightly less physical activity and slightly more screen time than other estimates; however, the difference (in screen time at least), may be because ISCOLE data collection has occurred more recently, and may not reflect a true difference. The proportion of ISCOLE participants considered overweight or obese was much higher than previous estimates.

\section{Discussion}


This study aimed to compare data collected in ISCOLE to data collected via nationally representative studies in all ISCOLE study sites. Of the 12 ISCOLE countries, eight provided data on weight status (BMI), five provided data on objectively measured physical activity (steps per day), and nine provided data on self-reported screen time.

The five ISCOLE countries that were part of the HBSC survey provided additional data for self-reported physical activity (meeting physical activity guidelines). When data were available, mean data from ISCOLE study appears to be relatively similar to country-level data; however, this varied with data availability and quality, and there were no systematic differences across countries or variables. Few countries used the same cutpoints or measurement tools in their national studies as ISCOLE when analysing participant characteristics.

Initially, the aim of this study was to compare data from ISCOLE sites to nationally representative data in the site country, to get a crude evaluation of the potential bias in the ISCOLE sample. However, it quickly became apparent that many countries do not collect nationally representative data on physical activity, screen time, or weight status. When data were available, it was collected using different tools or with different methods. For example, we aimed to compare accelerometer-measured daily moderate- to vigorous-intensity physical activity (minutes per day); however, after scoping the literature for comparable country level data, this was not possible. Due to the range of different models of accelerometers, and the different cut-points used to distinguish activity intensity, only two countries (the U.S., and Canada) were able to provide comparable data. No countries that could provide nationally representative 
accelerometer measured physical activity data used common cut-points. Therefore, we opted to examine physical activity via steps per day, which can be measured using an accelerometer, or a pedometer. The added benefit of examining steps per day is that the cost of pedometers is quite low, making it more feasible to use them in large, population based studies, or in times where resources are limited.

This work has several strengths and limitations. Many countries could not provide any comparable data and therefor it was difficult for us to determine if ISCOLE participant characteristics were similar to other children. This meant that many comparisons could not be made, and in most cases we did not have access to the raw data from nationally representative surveys and could not complete any formal statistical analysis. This leaves our comparisons open to interpretation, and our judgement on similarities (or differences) in the data open to criticism. However, we believe this also highlights an important limitation of international research programs the inconsistencies in measurement tools and cut points across different research centres, and countries, and the unavailability of raw data. Most of the studies included in this work used different methodologies, and different cut points to assess similar health variables. For example, BMI is a common measure of adiposity in children, yet there are four different cut points used, all of which provide different prevalence of overweight/obesity. ${ }^{43}$ ISCOLE recruitment was also limited to urban and suburban schools, and therefore we cannot account for populations living in rural areas. Previous work has consistently shown differences in obesity and lifestyle habits between these two groups, and that these differences may be most important in developing areas. ${ }^{44-46}$ 
The results of this work provide a valuable summary when developing future research programs and can help inform public health interventions. Another strength of this work is related to the rigour of the ISCOLE data collection and management procedures. ${ }^{1}$ The ISCOLE framework and coordinating center ensured all study sites, and all ISCOLE researchers, completed mandatory training for all aspects of the study..

\section{Conclusions}

This manuscript was designed as a methodological and ecological comparison study that may be used to provide evidence of the potential bias from each ISCOLE country sample, facilitating future intra- and inter-country comparisons. Due to the limited availability of country-level data, it is suggested that ISCOLE data be used with appropriate caution when planning countrywide population health interventions. However, for many countries ISCOLE currently provides the most up-to-date, most robust, and sometimes the only data on obesity and physical activity in children. This work has identified the paucity of comparable country data around the world and highlighted the importance of large, multi-national studies like ISCOLE. Moving forward, we recommend that researchers harmonize procedures for data collection and analysis. It is important to use the momentum, and collaborations that were built in ISCOLE to inform public health interventions, as well as other cross-sectional, surveillance surveys. 


\section{Acknowledgements}

We wish to thank the ISCOLE External Advisory Board and the ISCOLE participants and their families who made this study possible. The ISCOLE Research Group includes: Coordinating Center, Pennington Biomedical Research Center: Peter T. Katzmarzyk, PhD (Co-PI), Timothy S. Church, MD, PhD (Co-PI), Denise G. Lambert, RN (Project Manager), Tiago Barreira, PhD, Stephanie Broyles, PhD, Ben Butitta, BS, Catherine Champagne, PhD, RD, Shannon Cocreham, MBA, Kara Dentro, MPH, Katy Drazba, MPH, Deirdre Harrington, PhD, William Johnson, PhD, Dione Milauskas, MS, Emily Mire, MS, Allison Tohme, MPH, Ruben Rodarte MS, MBA; Data Management Center, Wake Forest University: Bobby Amoroso, BS, John Luopa, BS, Rebecca Neiberg, MS, Scott Rushing, BS; Australia, University of South Australia: Timothy Olds, PhD (Site Co-PI), Carol Maher, PhD (Site Co-PI), Lucy Lewis, PhD, Katia Ferrar, B Physio (Hon), Effie Georgiadis, BPsych, Rebecca Stanley, BAppSc (OT) Hon; Brazil, Centro de Estudos do Laboratório de Aptidão Física de São Caetano do Sul (CELAFISCS): Victor Keihan Rodrigues Matsudo, MD, PhD (Site PI), Sandra Matsudo, MD, PhD, Timoteo Araujo, MSc, Luis Carlos de Oliveira, MSc, Leandro Rezende, BSc, Luis Fabiano, BSc, Diogo Bezerra, BSc, Gerson Ferrari, MSc; Canada, Children's Hospital of Eastern Ontario Research Institute: Mark S. Tremblay, PhD (Site Co-PI), Jean-Philippe Chaput, PhD (Site Co-PI), Priscilla Bélanger, MA, Mike Borghese, MSc, Charles Boyer, MA, Allana LeBlanc, MSc, Claire Francis, M.Sc.,Geneviève Leduc, PhD; China, Tianjin Women's and Children's Health Center: Pei Zhao, MD (Site Co-PI), Gang Hu, MD, PhD (Site Co-PI), Chengming Diao, MD, Wei Li, MD, Weiqin Li, MSc, Enqing Liu, MD, Gongshu Liu, MD, Hongyan Liu, MD, Jian Ma, MD, Yijuan Qiao, MD, Huiguang Tian, PhD, Yue Wang, MD, Tao Zhang, MSc, Fuxia Zhang, MD; Colombia, Universidad de los Andes: Olga Sarmiento, MD, PhD (Site PI), Julio Acosta, Yalta Alvira, BS, Maria Paula Diaz, Rocio Gamez, BS, Maria Paula Garcia, Luis Guillermo Gómez, Lisseth Gonzalez, Silvia Gonzalez, RD, Carlos Grijalba, MD, Leidys Gutierrez, David Leal, Nicolas Lemus, Etelvina Mahecha, BS, Maria Paula Mahecha, Rosalba Mahecha, BS, Andrea Ramirez, MD, Paola Rios, MD, Andres Suarez, Camilo Triana; Finland, University of Helsinki: Mikael Fogelholm, ScD (Site-PI), Elli Hovi, BS, Jemina Kivelä, Sari Räsänen, BS, Sanna Roito, BS, Taru Saloheimo, MS, Leena Valta; India, St. Johns Research Institute: Anura Kurpad, MD, PhD (Site Co-PI), Rebecca Kuriyan, PhD (Site Co-PI), Deepa P. Lokesh, BSc, Michelle Stephanie D'Almeida, BSc, Annie Mattilda R, MSc, Lygia Correa, BSc, Vijay D, BSc; Kenya, Kenyatta University: Vincent Onywera, PhD (Site Co-PI), Mark S. Tremblay, PhD (Site Co-PI), Lucy-Joy Wachira, PhD, Stella Muthuri, PhD; Portugal, University of Porto: Jose Maia, PhD (Site PI), Alessandra da Silva Borges, BA, Sofia Oliveira Sá Cachada, Msc, Raquel Nichele de Chaves, MSc, Thayse Natacha Queiroz Ferreira Gomes, MSc, Sara Isabel Sampaio Pereira, BA, Daniel Monteiro de Vilhena e Santos, PhD, Fernanda Karina dos Santos, MSc, Pedro Gil Rodrigues da Silva, BA, Michele Caroline de Souza, MSc; South Africa, University of Cape Town: Vicki Lambert, PhD (Site PI), Matthew April, BSc (Hons), Monika Uys, BSc (Hons), Nirmala Naidoo, MSc, Nandi Synyanya, Madelaine Carstens, BSc(Hons); United Kingdom, University of Bath: Martyn Standage, PhD (Site PI), Sean Cumming, PhD, Clemens Drenowatz, PhD, Lydia Emm, MSc, Fiona Gillison, PhD, Julia Zakrzewski, PhD; United 
States, Pennington Biomedical Research Center: Catrine Tudor-Locke, PhD (Site-PI), Ashley Braud, Sheletta Donatto, MS, LDN, RD, Corbin Lemon, BS, Ana Jackson, BA, Ashunti Pearson, MS, Gina Pennington, BS, LDN, RD, Daniel Ragus, BS, Ryan Roubion, John Schuna, Jr., PhD; Derek Wiltz. The ISCOLE External Advisory Board includes Alan Batterham, PhD, Teesside University, Jacqueline Kerr, PhD, University of California, San Diego; Michael Pratt, MD, Centers for Disease Control and Prevention, Angelo Pietrobelli, MD, Verona University Medical School.

Conflict of interest: ISCOLE was funded by The Coca-Cola Company. The funder had no role in the design and conduct of the study; collection, management, analysis and interpretation of the data; and preparation, review or approval of the manuscript. The authors declare no other conflicts of interest. 
Table 1: ISCOLE country characteristics

\begin{tabular}{|c|c|c|c|c|c|}
\hline Country & $\begin{array}{l}\text { National } \\
\text { population }^{a}\end{array}$ & $\begin{array}{l}\text { ISCOLE site } \\
\text { location }\end{array}$ & $\begin{array}{l}\text { Population of ISCOLE } \\
\text { site location }{ }^{b}\end{array}$ & World bank classification ${ }^{c}$ & $\begin{array}{l}\text { Large and/or } \\
\text { National } \\
\text { study/studies }\end{array}$ \\
\hline \multicolumn{6}{|l|}{ Europe } \\
\hline Finland & $5,442,322$ & $\begin{array}{l}\text { Helsinki, } \\
\text { Espoo, Vantaa }\end{array}$ & $1,060,701$ & High-income & HBSC \\
\hline $\begin{array}{l}\text { United } \\
\text { Kingdom }\end{array}$ & $63,705,000$ & $\begin{array}{l}\text { Bath, North } \\
\text { East Somerset }\end{array}$ & 177,700 & High-income & $\begin{array}{l}\text { ENERGY, HBSCd, } \\
\text { HELENA, IDEFICS, } \\
\text { MCS }\end{array}$ \\
\hline Portugal & $10,562,178$ & Porto & 237,584 & High-income & EYHS $^{\dagger}, \mathrm{HBSC}$ \\
\hline \multicolumn{6}{|l|}{ Africa } \\
\hline Kenya & $44,354,000$ & Nairobi & $3,138,369$ & Low-income & None available \\
\hline South Africa & $52,981,991$ & Cape Town & $3,497,097$ & Upper-middle-income & $\begin{array}{l}\text { Time of Use } \\
\text { survey }\end{array}$ \\
\hline \multicolumn{6}{|l|}{ The Americas } \\
\hline Canada & $35,158,304$ & Ottawa & 883,391 & High-income & CHMS, HBSC \\
\hline United States & $316,783,000$ & Baton Rouge & 802,484 & High-income & $\begin{array}{l}\text { HBSC, NHANES, } \\
\text { YRBS }\end{array}$ \\
\hline Colombia & $47,262,816$ & Bogotá & $7,674,366$ & Upper-middle-income & ENSIN \\
\hline Brazil & $201,032,714$ & $\begin{array}{l}\text { Sao Caetano } \\
\text { do Sul }\end{array}$ & 149,263 & Upper-middle-income & BIGS \\
\hline \multicolumn{6}{|l|}{ South Asia } \\
\hline India & $1,242,456,566$ & Bangalore & $9,588,910$ & Lower-middle-income & None available \\
\hline \multicolumn{6}{|c|}{ Western Pacific } \\
\hline China & $1,362,620,526$ & Tianjin & $10,290,987$ & Upper-middle-income & CNNS \\
\hline Australia & $23,235,207$ & Adelaide & $1,212,982$ & High-income & AHS, ANCNPAS \\
\hline
\end{tabular}

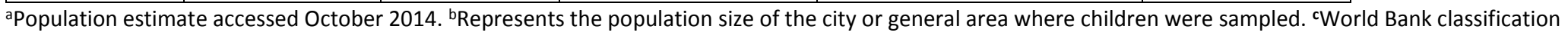
represents. ${ }^{\mathrm{d}}$ Since the U.K. ISCOLE site was in England, the corresponding HBSC data was taken from HBSC England site.

AHS: Australian Health Survey; ANCNPAS: Australian National Children's Nutrition and Physical Activity Survey; BIGS: Brazilian Institute of Geography and Statistics; CNNS: China National Nutrition Survey; CHMS: Canadian Health Measures Survey; ENERGY: EuropeaN Energy balance Research to prevent excessive weight Gain among Youth; ENSIN: Encuesta Nacional de la Situación Nutricional (National Survey of Nutritional Status); IDEFICS: identification and prevention of dietary- and

lifestyle induced health effects in children and infants; HBSC: Health Behavior in School-aged Children; HELENA: Healthy Lifestyle in Europe by Nutrition in Adolescents; MCS: Millennium Cohort Study; NHANES: National Health and Nutrition Examination Survey. 
Table 2: ISCOLE participant characteristics

\begin{tabular}{|c|c|c|c|c|c|}
\hline Study site & $\begin{array}{l}\text { Participants } \\
\text { ( } n, \% \text { boys) }\end{array}$ & $\begin{array}{l}\text { Age (years) } \\
\text { (mean, SD) }\end{array}$ & Weight status (\%)a & $\begin{array}{l}\text { Combined annual household } \\
\text { income }^{b}\end{array}$ & $\begin{array}{l}\text { Approximate equivalent in } \\
\text { U.S. dollars }{ }^{c}\end{array}$ \\
\hline \multicolumn{6}{|l|}{ Europe } \\
\hline Finland & $536(47.2 \%)$ & $10.0(0.4)$ & $\begin{array}{l}\text { Normal weight: } 74.6 \\
\text { Overweight/obese: } 23.7\end{array}$ & $\begin{array}{l}\text { Less than } 20,000 €: 5.5 \% \\
80,000 € \text { and above: } 40.9 \%\end{array}$ & $\begin{array}{l}\$ 26,000: 5.5 \% \\
\$ 104,000 \text { and above: } 40.9 \%\end{array}$ \\
\hline United Kingdom & $525(45.1 \%)$ & $10.4(0.5)$ & $\begin{array}{l}\text { Normal weight: } 68.5 \\
\text { Overweight/obese: } 30.3\end{array}$ & $\begin{array}{l}\text { Less than } £ 10,000: 9.4 \% \\
£ 10,000-£ 19,999: 17.3 \% \\
£ 90,000 \text { and above: } 8.1 \%\end{array}$ & $\begin{array}{l}\text { Less than } 16,500: 9.4 \% \\
\$ 16,500-\$ 32,998: 17.3 \% \\
\$ 148,500 \text { and above: } 8.1 \%\end{array}$ \\
\hline Portugal & $777(46.1 \%)$ & $10.0(0.3)$ & $\begin{array}{l}\text { Normal weight: } 52.5 \\
\text { Overweight/obese: } 47.2\end{array}$ & $\begin{array}{l}\text { Under } € 6,000: 20.5 \% \\
€ 6,000-€ 11,999: 30.9 \% \\
€ 42,000 \text { and above: } 5.5 \%\end{array}$ & $\begin{array}{l}\text { Under } \$ 7,800: 20.5 \% \\
\$ 7,800-15,599: 30.9 \% \\
\$ 54,6000 \text { and above: } 5.5 \%\end{array}$ \\
\hline \multicolumn{6}{|l|}{ Africa } \\
\hline Kenya & $563(46.5 \%)$ & $9.8(0.7)$ & $\begin{array}{l}\text { Normal weight: } 75.1 \\
\text { Overweight/obese: } 21.1\end{array}$ & $\begin{array}{l}\text { Less than Ksh. } 121,980: 23.2 \% \\
\text { Ksh } 6,000,000 \text { and above: } 3.5 \%\end{array}$ & $\begin{array}{l}\text { Less than } \$ 1,342: 23.2 \% \\
\$ 66,000 \text { and above: } 3.5 \%\end{array}$ \\
\hline South Africa & $550(40.1 \%)$ & $9.8(0.7)$ & $\begin{array}{l}\text { Normal weight: } 71.2 \\
\text { Overweight/obese: } 26.4\end{array}$ & $\begin{array}{l}\text { Less than R11,500: } 47.8 \% \\
\text { More than R500,000: } 7.6 \%\end{array}$ & $\begin{array}{l}\text { Less than } \$ 1,081: 47.8 \% \\
\$ 47,000 \text { and above: } 7.6 \%\end{array}$ \\
\hline \multicolumn{6}{|l|}{ The Americas } \\
\hline Canada & $565(42.2 \%)$ & $10.0(0.4)$ & $\begin{array}{l}\text { Normal weight: } 68.7 \\
\text { Overweight/obese: } 30.8\end{array}$ & $\begin{array}{l}\text { Less than } \$ 14,999: 2.9 \% \\
\$ 140,000 \text { and above: } 38.4 \%\end{array}$ & $\begin{array}{l}\text { Less than } \$ 13,799: 2.9 \% \\
\$ 128,800 \text { and above: } 38.4 \%\end{array}$ \\
\hline United States & $651(43.2 \%)$ & $9.5(0.6)$ & $\begin{array}{l}\text { Normal weight: } 58.4 \\
\text { Overweight/obese: } 41.3\end{array}$ & $\begin{array}{l}\text { Less than } \$ 10,000: 20.4 \% \\
\$ 140,000 \text { and above: } 21.6 \%\end{array}$ & N/A \\
\hline Colombia & $919(49.4 \%)$ & $10.0(0.6)$ & $\begin{array}{l}\text { Normal weight: } 75.7 \\
\text { Overweight/obese: } 22.9\end{array}$ & $\begin{array}{l}\$ 0-\$ 1.200 .000: 0.7 \% \\
\$ 4.800 .000-\$ 8.400 .000: 29.4 \% \\
\$ 36.000 .000 \text { and above: } 8.8 \%\end{array}$ & $\begin{array}{l}\$ 0-\$ 624: 0.7 \% \\
\$ 2,496-\$ 4,368: 29.4 \% \\
\$ 18,720 \text { and above: } 8.8 \%\end{array}$ \\
\hline Brazil & $584(49.1 \%)$ & $10.1(0.5)$ & $\begin{array}{l}\text { Normal weight: } 52.8 \\
\text { Overweight/obese: } 45.2\end{array}$ & $\begin{array}{l}\text { Under R 6.54,00: } 3.1 \% \\
\text { R\$6.540,00 - R\$19.620,00: } 35.1 \% \\
\text { R: } 85.020,01 \text { and above: } 4.4 \%\end{array}$ & $\begin{array}{l}\text { Less than } \$ 2,943: 3.1 \% \\
\$ 2,943-\$ 8,829: 35.1 \% \\
\$ 38,259 \text { and above: } 4.4 \%\end{array}$ \\
\hline \multicolumn{6}{|l|}{ South Asia } \\
\hline India & $620(47.1 \%)$ & $10.0(0.6)$ & $\begin{array}{l}\text { Normal weight: } 61.5 \\
\text { Overweight/obese: } 33.7\end{array}$ & $\begin{array}{l}\text { Less than Rs } 60000: 2.8 \% \\
\text { Rs720000 - and above: } 37.6 \%\end{array}$ & $\begin{array}{l}\text { Less than } \$ 1,020: 2.8 \% \\
\$ 12,240 \text { and above: } 37.6 \%\end{array}$ \\
\hline \multicolumn{6}{|l|}{ Western Pacific } \\
\hline China & $552(53.1 \%)$ & $9.4(0.5)$ & $\begin{array}{l}\text { Normal weight: } 56.3 \\
\text { Overweight/obese: } 41.2\end{array}$ & $\begin{array}{l}\text { Less than } ¥ 20,000: 18.1 \% \\
¥ 20,000-¥ 39,999: 18.1 \% \\
¥ 150,000 \text { and above: } 10.4 \%\end{array}$ & $\begin{array}{l}\text { Less than } \$ 3,200: 18.1 \% \\
\$ 3,200-\$ 6,400: 18.1 \% \\
\$ 24,000 \text { and above: } 10.4 \%\end{array}$ \\
\hline Australia & $528(46.0 \%)$ & $10.3(0.5)$ & $\begin{array}{l}\text { Normal weight: } 61.4 \\
\text { Overweight/obese: } 37.9\end{array}$ & $\begin{array}{l}\text { Less than } \$ 10,000: 2.1 \% \\
\$ 70,000 \text { to } \$ 89,999: 17.0 \% \\
\$ 140,000 \text { and above: } 21.7 \%\end{array}$ & $\begin{array}{l}\text { Less than } \$ 9,300: 2.1 \% \\
\$ 65,100-\$ 83,699: 17.0 \% \\
\$ 130,200 \text { and above: } 21.7 \%\end{array}$ \\
\hline
\end{tabular}

aWeight status defined by World Health Organization cut-points. ${ }^{43}$ bPresented as percent of participants in the lowest, highest, and median income categories. If the median income category was also the lowest, or the highest, only two income categories are presented see Figure 5 for income distribution and Additional file 1 for additional information. 'Currency conversion reflects rates from 2014. 


\section{Figure legends}

\section{Figure 1}

Title: Proportion of children considered overweight or obese from ISCOLE study sites and their representative countries.

Legend: Dark grey bars indicate data from ISCOLE participants; white bars represent country-level data. If no white bar, then country level data are not available. Where available, data are presented for both boys and girls. Data were analyzed as per BMI cut-points available in each country as follows. World Health Organization: Portugal, Colombia, Brazil; International Obesity Task Force: Canada, Australia; Center for Disease Control and Prevention: United States; other: United Kingdom (British 1990 growth reference ${ }^{20}$ ), China (China BMI criteria, overweight $\geq 19.4$, obese $\geq 22.2$ ). ${ }^{47}$ Country level datasets included: U.K.: National Child Measurement Programme; ${ }^{20}$ Portugal: Plataforma Contra a Obesidade; ${ }^{48}$ Canada: Canadian Health Measures Survey; ${ }^{49}$ U.S., National Health and Nutrition Examination Survey; Colombia: Encuesta Nacional de la Situación Nutricional; Brazil: Brazilian Institute of Geography and Statistics; ${ }^{28}$ China: China Health and Nutrition Survey; ${ }^{47}$ Australia: Australian National Children's Nutrition and Physical Activity Survey. ${ }^{38}$ BMI: Body Mass Index. See Additional file 3 for additional study details for country level data.

\section{Figure 2}

Title: Daily physical activity (steps per day) from ISCOLE study sites and their representative countries 
Legend: Dark grey bars indicate data from ISCOLE participants; white bars represent country-level data. If no white bar, then country level data were not available. Where available, data are presented for both boys and girls. Horizontal black line represents mean steps per day for all ISCOLE participants; horizontal dashed line represents target of 12,000 steps per day recommended to meet current physical activity guidelines. ${ }^{50}$ Data were included if it was collected via pedometer or accelerometer, and presented as sample mean. Country level datasets included: Finland: Physical Activity of School Aged Children; ${ }^{51}$ United Kingdom: Millennium Cohort Study; ${ }^{19}$ Canada: Canadian Health Measures Survey; ${ }^{31}$ U.S.: National Health and Nutrition Examination Survey; ${ }^{52}$ Australia:Australian National Children's Nutrition and Physical Activity Survey. ${ }^{38}$ The Millennium Cohort Study also provided data for England: 10,147 steps per day compared to 9982 steps per day in ISCOLE.See Additional file 4 for additional study details for country level data.

\section{Figure 3}

Title: Proportion of children exceeding screen time guidelines ( $>2$ hours per day) from ISCOLE sites and their representative countries.

Legend: Dark grey bars indicate data from ISCOLE participants; white bars represent country-level data. If no white bar, then country level data were not available. Where available, data are presented for both boys and girls. Horizontal black like represents mean proportion of all ISCOLE participants who exceed screen time guidelines. Country level datasets included: Canada, Finland, the U.K., and the U.S.: Healthy Behaviors in 
School-aged Children; ${ }^{3}$ South Africa: Time of Use survey; ${ }^{26}$ Colombia: Instituto Colombiano de Bienestar Familiar (ICBF); China: China Health and Nutrition Survey $;{ }^{53}$ Australia: Australian Bureau of Statistics. ${ }^{39}$ See Additional file 5 for additional study details for country level data.

\section{Figure 4}

Title: Proportion of girls (Panel A) and boys (Panel B) who self-reported that they engage in at least 60 minutes of moderate- to vigorous-intensity physical activity every day of the week.

Legend: Dark grey bars indicate data from ISCOLE participants; white bars represent data that were adapted from the HBSC survey. ${ }^{4}$ MVPA: moderate- to vigorous-intensity physical activity. 


\section{References}

1 Katzmarzyk PT, Barreira TV, Broyles ST, Champagne CM, Chaput J-P, Fogelholm M et al. The International Study of Childhood Obesity, Lifestyle and the Environment (ISCOLE): design and methods. BMC Public Health 2013; 13: 900.

$2 \quad$ Healthy Lifestyle in Europe by Nutrition in Adolescence. http://www.helenastudy.com/.

3 Currie C, Gabhainn SN, Godeau E, Roberts RS, Currie O, Picket W et al. Inequalities in Young People's Health: Health Behaviour in School-aged Children. International Report from the 20052006 Survey Health Policy for Children and Adolescents. 2008.

4 Currie C, Zanottie C, Morgan A, Currie D, de Looze M, Roberts C et al. Social determinants of health and well-being among young people. World Health Organization: Copenhagen, WHO Regional Office for Europe, 2012.

5 Barreira TV, Schuna JM, Mire EF, Katzmarzyk PT, Chaput J-P, Leduc G et al. Identifying Children's Nocturnal Sleep Using 24-h Waist Accelerometry. Med Sci Sports Exerc 2014. doi:10.1249/MSS.0000000000000486.

6 Trost SG, Loprinzi PD, Moore R, Pfeiffer KA. Comparison of Accelerometer Cut Points for Predicting Activity Intensity in Youth: Med Sci Sports Exerc 2011; 43: 13601368.

7 Colley RC, Connor Gorber S, Tremblay MS. Quality control and data reduction procedures for accelerometry-derived measures of physical activity. Health Rep 2010;

21: 63-69. 
8 Evenson KR, Catellier DJ, Gill K, Ondrak KS, McMurray RG. Calibration of two objective measures of physical activity for children. J Sports Sci 2008; 26: 1557-1565. $9 \quad$ Youth Risk Behavior Surveillance System (YRBSS). US Cent. Dis. Control Prev. 2012.

10 Okely AD, Salmon J, Trost SG, Hinkley T. Discussion paper for the development of physical activity recommendations for children under 5 years. Australian Government Department of Health and Ageing: Australia, 2008.

11 Tremblay MS, Warburton DER, Janssen I, Paterson DH, Latimer AE, Rhodes RE et al. New Canadian Physical Activity Guidelines. Appl Physiol Nutr Metab 2011; 36: 36-46.

12 Tremblay MS, LeBlanc AG, Janssen I, Kho ME, Hicks A, Murumets K et al. Canadian Sedentary Behaviour Guidelines for Children and Youth. Appl Physiol Nutr Metab 2011; 36: 59-64.

13 Bull FC, Expert Working Group. Physical activity guidelines in the U.K.: review and recommendations. School of Sport, Exercise, and Health Sciences, Loughborough University: Leichestershire, United Kingdom, 2010.

14 Pate RR PM. Physical activity and public health: A recommendation from the centers for disease control and prevention and the american college of sports medicine. JAMA 1995; 273: 402-407.

15 World Health Organization. Global recommendations on physical activity for health. 2010http://www.who.int/dietphysicalactivity/publications/9789241599979/en/index.ht ml (accessed 28 Feb2013). 
16 Dept of Sport Sciences, University of Jyväskylä, Finland., Liukkonen J, Jaakkola T, Kokko S, Gråstén A, Yli-Piipari S et al. Results From Finland's 2014 Report Card on Physical Activity for Children and Youth. J Phys Act Health 2014; 11: S51-S57.

17 Dept for Health, University of Bath., Standage M, Wilkie HJ, Jago R, Foster C, Goad MA et al. Results From England's 2014 Report Card on Physical Activity for Children and Youth. J Phys Act Health 2014; 11: S45-S50.

18 Plataforma Contra a Obesidade.

http://www.plataformacontraaobesidade.dgs.pt/PresentationLayer/homepage_instituci onal.aspx?menuid=113.

19 Griffiths L, Cortina-Borja M, Sera F, Pouliou T, Geraci M, Rich C et al. How active are our children? Findings from the Millennium Cohort Study. BMJ Open 2013; 3:

e002893.

20 Lifestyle Statistics Team. National Child Measurement Programme: England, 2012/2013 school year. Health and Social Care Information Centre, Public Health England, 2013.

21 Kenyatta University, Nairobi, Kenya., Wachira L JM, Muthuri SK, Tremblay MS, Onywera VO. Results From Kenya's 2014 Report Card on the Physical Activity and Body Weight of Children and Youth. J Phys Act Health 2014; 11: S69-S73.

22 MRC/UCT Research Unit for Exercise Science and Sports Medicine, Dept of Human Biology, University of Cape Town, Draper C, Basset S, de Villiers A, Lambert EV, Writing Group H. Results From South Africa's 2014 Report Card on Physical Activity for Children and Youth. J Phys Act Health 2014; 11: S98-S104. 
23 Muthuri SK, Wachira L-JM, Leblanc AG, Francis CE, Sampson M, Onywera VO et al. Temporal Trends and Correlates of Physical Activity, Sedentary Behaviour, and Physical Fitness among School-Aged Children in Sub-Saharan Africa: A Systematic Review. Int J Environ Res Public Health 2014; 11: 3327-3359.

24 Muthuri SK, Francis CE, Wachira L-JM, LeBlanc AG, Sampson M, Onywera VO et al. Evidence of an Overweight/Obesity Transition among School-Aged Children and Youth in Sub-Saharan Africa: A Systematic Review. PLoS ONE 2014; 9: e92846.

25 Onywera VO, Adamo KB, Sheel AW, Waudo JN, Boit MK, Tremblay M. Emerging Evidence of the Physical Activity Transition in Kenya. J Phys Act Health 2012; 9: 554-562. 26 Statistics South Africa. A time of use. Statistics South Africa: Pretoria, South Africa, 2013.

27 Giroux S. Canadian Health Measures Survey: sampling strategy overview. Health Rep 2006; 18 Suppl: 31-36.

28 Brazilian Institute of Geography and Statistics (Instituto Brasileiro de Geografia e Estatística). http://www.ibge.gov.br/english/.

29 Healthy Active Living and Obesity Research Group, Gray CE, Barnes JD, Cowie Bonne J, Cameron C, Chaput JP et al. Results From Canada's 2014 Report Card on Physical Activity for Children and Youth. J Phys Act Health 2014; 11: S26-S32.

30 Active Healthy Kids Canada. Are we driving our kids to unhealthy habits? The 2013 Active Healthy Kids Canada Report Card on Physical Activity for children and youth. 2013. 
31 Colley RC, Garriguet D, Janssen I, Craig CL, Clarke J, Tremblay MS. Physical activity of Canadian children and youth: Accelerometer results from the 2007 to 2009 Canadian Health Measures Survey. Health Rep 2011; 22: 15-23.

32 Instituto Colombiano de Bienestar Familiar ICBF. Encusesta Nacional de la Situacion Nutricional en Colombia ENSIN. Insituto Colombiano de Bienestar Familiar, Ed: Bogota, 2005.

33 Gomez LF, Parra DC, Lobelo F, Samper B, Moreno J, Jacoby E et al. Television viewing and its association with overweight in Colombian children: results from the 2005 National Nutrition Survey: A cross sectional study. Int J Behav Nutr Phys Act 2007;

\section{4: 41.}

34 Troiano RP, Berrigan D, Dodd KW, Mâsse LC, Tilert T, McDowell M. Physical activity in the United States measured by accelerometer. Med Sci Sports Exerc 2008; 40: $181-188$.

35 Pennington Biomedical Research Center, Dentro KN, Beals K, Crouter SE, Eisenmann JC, McKenzie TL et al. Results From the United States' 2014 Report Card on Physical Activity for Children and Youth. J Phys Act Health 2014; 11: S105-S112.

36 Barreira TV, Schuna JM, Mire EF, Broyles ST, Katzmarzyk PT, Johnson WD et al. Normative Steps/Day and Peak Cadence Values for United States Children and Adolescents: National Health and Nutrition Examination Survey 2005-2006. J Pediatr 2015; 166: 139-143.e3.

37 Expert panel on integrated guidelines for cardiovascular health and risk reduction in children and adolescents. Expert Panel on Integrated Guidelines for 
Cardiovascular Health and Risk Reduction in Children and Adolescents: Summary Report. Pediatrics 2011; 128: S213-S256.

38 Department of Health and Ageing. Australian National Children's Nutrition and Physical Activity Survey (ANCNPAS): main findings. Commonwealth Scientific and Industrial Research Organisation (CSIRO) and the University of South Australia: Canberra, Australia, 2008.

39 Australian Bureau of Statistics. Australian Health Survey: Physical Activity, 201112. 2013.http://www.abs.gov.au/ausstats/abs@.nsf/Lookup/4364.0.55.001main+features1 2011-12 (accessed 10 Oct2014).

40 University of South Australia., Schranz N, Olds T, Cliff D, Davern M, Engelen L et al. Results From Australia's 2014 Report Card on Physical Activity for Children and Youth. J Phys Act Health 2014; 11: S21-S25.

41 Zhang J, Seo D-C, Kolbe L, Middlestadt S, Zhao W. Associated Trends in Sedentary Behavior and BMI Among Chinese School Children and Adolescents in Seven Diverse Chinese Provinces. Int J Behav Med 2011; 19: 342-350.

42 Yao N. Weight status in Chinese children: maternal perceptions and child selfassessments. World J Pediatr 2012; 8: 129-135.

43 Shields M, Tremblay MS. Canadian childhood obesity estimates based on WHO, IOTF and CDC cut-points. Int J Pediatr Obes 2010; 5: 265-273. 
44 Joens-Matre RR, Welk GJ, Calabro MA, Russell DW, Nicklay E, Hensley LD. RuralUrban Differences in Physical Activity, Physical Fitness, and Overweight Prevalence of Children. J Rural Health 2008; 24: 49-54.

45 Plotnikoff RC, Mayhew A, Birkett N, Loucaides CA, Fodor G. Age, gender, and urban-rural differences in the correlates of physical activity. Prev Med 2004; 39: 11151125.

46 Onywera VO. Adiposity and physical activity among children in countries at different stages of the physical activity transition : Canada, Mexico and Kenya. 2013.http://reference.sabinet.co.za/sa_epublication_article/ajpherd_v19_n1_a12 (accessed 25 Apr2015).

47 He W, Li Q, Yang M, Jiao J, Ma X, Zhou Y et al. Lower BMI cutoffs to define overweight and obesity in China: Chinese BMI Cutoffs. Obesity 2015; : n/a-n/a. 48 Sardinha LB, Santos R, Vale S, Silva AM, Ferreira JP, Raimundo AM et al. Prevalence of overweight and obesity among Portuguese youth: a study in a representative sample of 10-18-year-old children and adolescents. Int J Pediatr Obes 2011; 6: e124-128.

49 Tremblay M, Shields M, Laviolette M, Craig C, Janssen I, Connor Gorber S. Fitness of Canadian children and youth: results from the 2007-2009 Canadian Health Measures Survey. Health Rep 2010; 21: 7-20.

50 Colley RC, Janssen I, Tremblay MS. Daily Step Target to Measure Adherence to Physical Activity Guidelines in Children. Med Sci Sports Exerc; 44: 977-982. 
51 Tammelin T, Laine K, Turpeinen S. Oppilaiden fyysinen aktiivisuus. [Physical Activity of School-aged children]. Liikunnan ja kansanterveyden edistämissäätiö LIKES [Sport and Health LIKES]: Jyväskylä, 2013.

52 Tudor-Locke C, Johnson WD, Katzmarzyk PT. Accelerometer-Determined Steps per Day in US Children and Youth: Med Sci Sports Exerc 2010; 42: 2244-2250.

53 Cao H, Qian Q, Weng T, Yuan C, Sun Y, Wang H et al. Screen time, physical activity and mental health among urban adolescents in China. Prev Med 2011; 53: 316320. 


\section{Supplementary files}

Supplementary file 1: Classification of household income by country 
U.S.

$\mathbf{1}=$ "Less than $\$ 10,000 "$

$2=$ "\$10,000 - \$29,999"

$3=" \$ 30,000-\$ 49,999 "$

$4=$ "\$ 50,000 - \$ 69,999"

$5=$ "\$70,000 - \$89,999"

$6=$ "\$ 90,000 - \$109,999"

$7=$ "\$110,000 - \$139,999"

8 = "\$ 140,000 and above"

U.K.

1="Less than $£ 10,000 "$

2="£10,000 - $£ 19,999 "$

3="£20,000 - $£ 29,999 "$

4="£30,000 - $£ 39,999 "$

$5=" £ 40,000-£ 49,999 "$

6="£50,000 - $£ 59,999 "$

$7=" £ 60,000-£ 69,999 "$

8="£70,000 - £79,999"

9="£80,000 - £89,999"

$10=" £ 90,000$ and above"

\section{AUSTRALIA (3)}

$\mathbf{1}=$ "Less than $\$ 10,000 "$

$\mathbf{2}="$ "\$10,000 - \$29,999"

$3=$ "\$ 30,000 - \$49,999"

$4=$ "\$ 50,000 - \$ 69,999"

$\mathbf{5}=$ "\$ 70,000 - \$ 89,999"

$6=" \$ 90,000-\$ 109,999 "$

$7=$ "\$ 110,000 - \$139,999"

8 = "\$ 140,000 and above"

\section{PORTUGAL}

1 = "Menos de $€ 6,000 "$

2 = "€ 6,000 - € 11,999"

$\mathbf{3}=$ "€ 12,000 - € 17,999"

4 = "€ 18,000 - € 23,999"

5 = "€ 24,000 - € 29,999"

$6=$ "€ 30,000 - € 35,999"

$7=$ "€ 36,000 - € 41,999"

8 = "€ 42,000 ou superior"

\section{SOUTH AFRICA}

$\mathbf{1}=$ "Less than R11,500"

2 = "Between 11,500-R19,000"

3 = "Between 19,000-R30,000"

4 = "Between R30,000-R65,000"

$\mathbf{5}=$ "Between R65,000-R100,000"

$\mathbf{6}=$ "Between R100,000-R300,000"

7 = "Between R300,000-R500,000"

8 = "More than R500,000"

\section{KENYA}

$\mathbf{1}=$ "Less than Ksh.121,980"

$\mathbf{2}=$ "Ksh. 121,992 - Ksh. 236,892"

$\mathbf{3}=$ "Ksh. 236,904 - Ksh. 351,804"

4 = "Ksh. 351,816 - Ksh. 466,716"

$5=$ "Ksh. 466,728 - Ksh. 599,988"

$6=$ "Ksh. 600,000 - Ksh. 1,199,988"

7 = "Ksh. 1,200,000 - Ksh.

$1,799,988$ "

8 = "Ksh. 1,800,000 - Ksh.

$3,599,988^{\prime \prime}$

9 = "Ksh. 3,600,000 - Ksh.

$5,999,988$ "

$10=$ "Ksh. 6,000,000 and above"

\section{COLOMBIA}

$1=$ "\$ $0-\$ 1.200 .000 "$

$2=" \$ 1.200 .000-\$ 4.800 .000 "$

$3=" \$ 4.800 .000-\$ 8.400 .000 "$

$4=" \$ 8.400 .000-\$ 12.000 .000 "$

$\mathbf{5}=$ "\$12.000.000-\$18.000.000"

$6=" \$ 18.000 .000-\$ 24.000 .000 "$

$7=$ "\$24.000.000 - \$36.000.000"

$8=$ "\$ 36.000 .000 en adelante"

BRAZIL

$1=$ "Menos que $\mathrm{R} \$ 6.540,00 "$

2 = "Entre R\$6.540,00 -

$\mathrm{R} \$ 19.620,00 "$

3 = "Entre $\mathrm{R} \$ 19.620,01$ -

R\$32.700,00"

4 = "Entre R\$32.700,01 -

R\$45.780,00"

$\mathbf{5}=$ "Entre $\mathrm{R} \$ 45.780,01$ -

R\$58.860,00"

6 = "Entre $R \$ 58.860,01$ -

R\$71.940,00"

7 = "Entre R\$71.940,01 -

\$85.020,00"

8 = "R\$85.020,01 ou Mais"

\section{CANADA}

$1=$ "Less than $\$ 14,999 "$

$2=$ "\$ 15,000 - \$29,999"

$3=$ "\$30,000 - \$39,999"

$4=" \$ 40,000-\$ 59,999 "$

$5=$ "\$ 60,000 - \$89,999"

$6=$ "\$ 90,000 - \$109,999"

$7=$ "\$110,000 - \$139,999"

$8=$ "\$ 140,000 and above"

\section{CHINA}

$\mathbf{1}=$ "Less than $¥ 20,000 "$
$\mathbf{2}=$ "¥20,000- $¥ 39,999 "$
$\mathbf{3}=" ¥ 40,000-¥ 59,999 "$
$\mathbf{4}=" ¥ 60,000-¥ 79,999 "$
$\mathbf{5}=" ¥ 80,000-¥ 99,999 "$
$\mathbf{6}=" ¥ 100,000-¥ 119,999 "$
$\mathbf{7}=" ¥ 120,000-¥ 149,999 "$
$\mathbf{8}=" ¥ 150,000-$ and above

INDIA

1 = "Less than Rs60000"

2 = "Rs60000 - Rs119988"

3 = "Rs120000 - Rs239988"

4 = "Rs240000 - Rs359988"

5 = "Rs360000 - Rs479988"

6 = "Rs480000 - Rs599988"

7 = "Rs600000 - Rs719988"

8 = "Rs720000 - and above"

\section{FINLAND}

$1=$ "Less than $20,000 € "$

$2=$ "20,000 - 24,999€"

3 = "25,000 - 29,999€"

4 = "30,000 - 39,999€"

$5=$ "40,000 - 49,999€"

$6=$ "50,000 - 59,999€"

7 = "60,000 - 69,999€"

8 = "70,000 - 79,999€"

9= "80,000€ and above" 
Supplementary file 2: Country level surveys

\begin{tabular}{|c|c|c|c|c|c|c|c|c|c|}
\hline Study & $\begin{array}{l}\text { Year } \\
\text { study } \\
\text { began }\end{array}$ & Purpose & $\begin{array}{l}\text { Number } \\
\text { of } \\
\text { countries }\end{array}$ & Region & $\begin{array}{l}\text { Number of } \\
\text { participants } \\
\text { (approximate) }\end{array}$ & $\begin{array}{l}\text { Age or } \\
\text { age } \\
\text { groups } \\
\text { (years) }\end{array}$ & $\begin{array}{l}\text { Frequency } \\
\text { of data } \\
\text { collection }\end{array}$ & Variables measured & $\begin{array}{l}\text { Designed to be } \\
\text { nationally } \\
\text { representative } \\
\text { (yes/no) }\end{array}$ \\
\hline ANCNPAS $^{1}$ & 2007 & $\begin{array}{l}\text { To assess food and } \\
\text { nutrient intake, physical } \\
\text { activity participation and } \\
\text { to measure weight, } \\
\text { height, and waist } \\
\text { circumference in } \\
\text { Australian children. }\end{array}$ & 1 & Australia & 4,487 & $2-16$ & $\begin{array}{l}\text { Cross- } \\
\text { sectional, } \\
\text { once }\end{array}$ & $\begin{array}{l}\text { Dietary intake (24- } \\
\text { hour food recall), } \\
\text { physical activity (48- } \\
\text { hour time use survey, } \\
\text { pedometer), } \\
\text { anthropometric } \\
\text { (height, weight, waist } \\
\text { circumference) }\end{array}$ & $\begin{array}{l}\text { Yes (except for } \\
\text { those living in } \\
\text { remote areas, } \\
\text { or of } \\
\text { Indigenous } \\
\text { origin) }\end{array}$ \\
\hline $\mathrm{CHMS}^{2,3}$ & 2007 & $\begin{array}{l}\text { To create national } \\
\text { baseline data on the } \\
\text { extent of such major } \\
\text { health concerns as } \\
\text { obesity, hypertension, } \\
\text { cardiovascular disease, } \\
\text { exposure to infectious } \\
\text { diseases, and exposure to } \\
\text { environmental } \\
\text { contaminants. The CHMS } \\
\text { will enable us to } \\
\text { determine relationships } \\
\text { between disease risk } \\
\text { factors and health status, } \\
\text { and to explore emerging } \\
\text { public health issues. }\end{array}$ & 1 & Canada & $\begin{array}{l}\text { 5,700 per } \\
\text { cycle } \\
\text { (planned) }\end{array}$ & $3-79$ & Biennial & $\begin{array}{l}\text { Physical (height, } \\
\text { weight, waist } \\
\text { circumference, } \\
\text { skinfolds, BP, blood } \\
\text { measures, } \\
\text { spirometery, urine } \\
\text { measures); fitness } \\
\text { (mCAFT), activity } \\
\text { (accelerometry); } \\
\text { household } \\
\text { questionnaire } \\
\text { (education status, } \\
\text { general health, } \\
\text { demographics, SB, PA, } \\
\text { dietary intake) }\end{array}$ & Yes \\
\hline CHNS & 1989 & $\begin{array}{l}\text { To examine the effects of } \\
\text { the health, nutrition, and } \\
\text { family planning policies } \\
\text { and programs } \\
\text { implemented by national } \\
\text { and local governments } \\
\text { and to see how the social } \\
\text { and economic } \\
\text { transformation of } \\
\text { Chinese society is } \\
\text { affecting the health and }\end{array}$ & 1 & China & 19,000 to date & All ages & $\begin{array}{l}\text { Not } \\
\text { specified }\end{array}$ & $\begin{array}{l}\text { Household survey; } \\
\text { adult survey (physical } \\
\text { activity, SES, } \\
\text { education, } \\
\text { occupation); child } \\
\text { survey (physical } \\
\text { activity, school/work } \\
\text { status, maturation); } \\
\text { nutrition survey (3-d } \\
\text { food recall, snack } \\
\text { frequency); } \\
\text { community survey }\end{array}$ & Yes \\
\hline
\end{tabular}




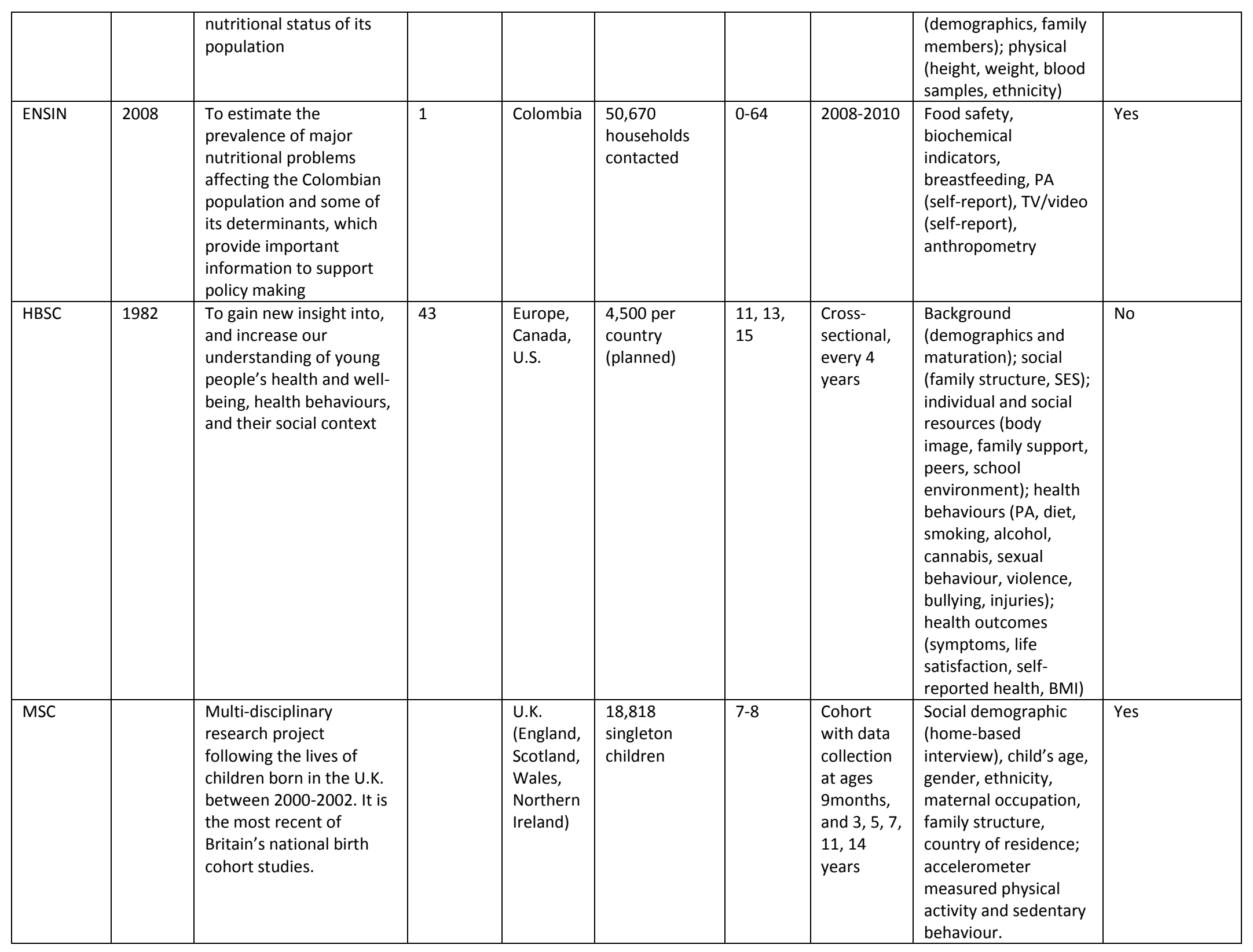




\begin{tabular}{|l|l|l|l|l|l|l|l|l|}
\hline NHANES & 1999 & $\begin{array}{l}\text { To assess the health and } \\
\text { nutritional status of } \\
\text { adults and children in the } \\
\text { United States }\end{array}$ & 1 & U.S. & $\begin{array}{l}\text { 5,000 per } \\
\text { cycle } \\
\text { (planned) }\end{array}$ & $>2$ & Biennial & $\begin{array}{l}\text { Family questionnaire } \\
\text { (demographic } \\
\text { background, } \\
\text { occupation, housing, } \\
\text { smoking, consumer } \\
\text { behaviour, income, } \\
\text { food security); sample } \\
\text { person questionnaire } \\
\text { (demographics, } \\
\text { physical } \\
\text { measurements, } \\
\text { occupation, health } \\
\text { care usage, } \\
\text { PA/fitness); diet (24-hr } \\
\text { food recall); PA } \\
\text { (accelerometer) }\end{array}$ \\
\hline
\end{tabular}

ANCNPAS: Australian National Children's Nutrition and Physical Activity Survey; CHMS: Canadian Health Measures Survey; CHNS: China Health and Nutrition Survey; ENERGY: EuropeaN Energy balance Research to prevent excessive weight Gain among Youth; ENSIN: Encuesta Nacional de la Situación Nutricional (National Survey of Nutritional Status); EYHS: European Youth Heart Study; IDEFICS: identification and prevention of dietary- and lifestyle induced health effects in children and infants; HBSC: Health Behaviour in School-aged Children; HELENA: Healthy Lifestyle in Europe by Nutrition in Adolescents; MSC: Millennium Cohort Study; NHANES: National Health and Nutrition Examination Survey.

*A study was deemed nationally representative if it was stated as such on the study website, or published protocol and/or explicit details on weighting of participants demographics was provided 
Supplementary file 3: Comparison of percent overweight or obese between ISCOLE sites and other national datasets using Body Mass Index

\begin{tabular}{|c|c|c|c|c|c|c|c|}
\hline \multirow[t]{2}{*}{ Study site } & \multicolumn{2}{|l|}{ ISCOLE sample } & \multicolumn{5}{|l|}{ National sample } \\
\hline & $\begin{array}{l}\text { Overweight/obese } \\
(\%)^{\mathrm{a}}\end{array}$ & $n, \%$ males & Dataset & $\begin{array}{l}\text { Age } \\
\text { (years) }\end{array}$ & $\mathrm{N}$ (\% males) & $\begin{array}{l}\text { Overweight/obese } \\
(\%, 95 \% \mathrm{Cl})\end{array}$ & Cut-point used \\
\hline \multicolumn{8}{|l|}{ Europe } \\
\hline Finland & $87(16.2)$ & $536(47.2)$ & - & - & - & - & - \\
\hline $\begin{array}{l}\text { U.K. } \\
\text { (England) }\end{array}$ & $\begin{array}{l}\text { Overweight: } 13.7 \\
\text { Obese: } 8.0\end{array}$ & $524(45.2)$ & $\begin{array}{l}\text { National Child } \\
\text { Measurement Program } \\
(2012-2013)^{¥}\end{array}$ & $10-11$ & $489,146(51.3)$ & $\begin{array}{l}\text { Overweight: } 14.4 \\
\text { Obese: } 18.9\end{array}$ & $\begin{array}{l}\text { British } 1990 \text { growth } \\
\text { reference }^{\text {b }}\end{array}$ \\
\hline Portugal $^{4}$ & $\begin{array}{l}\text { Boys:c } \\
\text { Overweight: } 16.5 \\
\text { Obese: } 34.9 \\
\text { Girls: }^{c} \\
\text { Overweight: } 17.7 \\
\text { Obese: } 24.6\end{array}$ & $777(46.1)$ & - & 10 & $1,001(48.6)^{4}$ & $\begin{array}{l}\text { Boys: } \\
\text { Overweight: } 29 \\
\text { Obese: } 21 \\
\text { Girls: } \\
\text { Overweight: } 28.0 \\
\text { Obese: } 17.3\end{array}$ & WHO \\
\hline \multicolumn{8}{|l|}{ Africa } \\
\hline Kenya & 16.0 & $563(46.5)$ & - & - & - & - & - \\
\hline $\begin{array}{l}\text { South } \\
\text { Africa }\end{array}$ & 21.2 & $550(40.1)$ & - & - & - & - & - \\
\hline \multicolumn{8}{|c|}{ The Americas } \\
\hline Canada $^{5}$ & $\begin{array}{l}\text { Boys: }^{\text {d }} \\
\text { Overweight: } 17.7 \\
\text { Obese: } 4.2 \\
\text { Girls: } \\
\text { Overweight: } 16.8 \\
\text { Obese: } 4.9\end{array}$ & $565(42.2)$ & CHMS (2007-2009) & $6-11$ & $878(51.1)$ & $\begin{array}{l}\text { Boys: } \\
\text { Overweight: } 17 \\
\text { Obese: } 14 \\
\text { Girls: } \\
\text { Overweight: } 16 \\
\text { Obese: } 10\end{array}$ & IOTF \\
\hline $\begin{array}{l}\text { United } \\
\text { States }^{6}\end{array}$ & $\begin{array}{l}\text { Boys: } 32.4 \\
\text { Girls: } 35.6\end{array}$ & $651(43.2)$ & NHANES (2011-2012) & $6-11$ & $1,268(51.3)$ & $\begin{array}{l}\text { Boys: } 33.2 \\
\text { Girls: } 35.2\end{array}$ & $\mathrm{CDC}$ \\
\hline Colombia & $23.25^{c}$ & $919(49.4)$ & ENSIN (2010) & $5-17$ & $49,877(51)$ & 17.5 & WHO \\
\hline Brazil & $45.74^{c}$ & $584(49.1)$ & BIGS & 10 & $127,853(52.3)$ & 33.1 & WHO \\
\hline \multicolumn{8}{|l|}{ South Asia } \\
\hline India & $\begin{array}{l}\text { Overweight: } 17.1 \\
\text { Obese: } 8.6\end{array}$ & $620(47.1)$ & - & - & - & - & - \\
\hline \multicolumn{8}{|c|}{ Western Pacific } \\
\hline China $^{7}$ & $\begin{array}{l}\text { Overweight: } 14.5 \\
\text { Obese: } 20.5\end{array}$ & $552(53.1)$ & CHNS & 10 & $8,977(50.0)$ & $\begin{array}{l}\text { Overweight: } 12.84 \\
\text { Obese: } 8.52\end{array}$ & See below ${ }^{e}$ \\
\hline Australia & $26.7^{\mathrm{d}}$ & $528(46.0)$ & ANCNPAS (2007) & $9-11$ & 538 & 28.4 & IOTF \\
\hline
\end{tabular}


${ }^{1}$ using the CDC cut-points unless otherwise noted ${ }^{b}$ Prevalence rates were calculated by deriving every child's BMI and referencing the age and sex specific centiles calculated using the British 1990 growth reference ${ }^{8}$. CUsing WHO criteria. dUsing IOTF criteria. e China BMI criteria: Overweight: 19.4, Obese: 22.2.

ANCNPAS: Australian National Children's Nutrition and Physical Activity Survey; BIGS: Brazilian Institute of Geography and Statistics; CDC: Center for Disease Control and Prevention; CHMS: Canadian Health Measures Survey; CHNS: China Health and Nutrition Survey; ENSIN: Encuesta Nacional de la Situación Nutricional (National Survey of Nutritional Status); IOTF: International Obesity Task Force; NHANES: National Health and Nutrition Examination Survey; WHO: World Health Organization; U.K.: United Kingdom. 
Supplementary file 4: Minutes of moderate- to vigorous intensity physical activity, step counts, and proportion of children meeting physical activity guidelines in ISCOLE and national studies

\begin{tabular}{|c|c|c|c|c|c|c|c|}
\hline \multirow[t]{2}{*}{ Study site } & \multicolumn{2}{|l|}{ ISCOLE Sample } & \multicolumn{5}{|l|}{ National Sample } \\
\hline & Physical activitya, b & $\mathrm{n}$ (\% boys) & Dataset & Age & $\mathrm{n}$ (\% males) & Physical activityc & $\begin{array}{l}\text { Accelerometer } \\
\text { cut-point used }\end{array}$ \\
\hline \multicolumn{8}{|l|}{ Europe } \\
\hline Finland & $\begin{array}{l}70.7 \mathrm{~min} / \text { day } \\
10,489 \text { steps/day } \\
\text { Meeting guidelines } \\
\text { Boys: } 22 \% \\
\text { Girls: } 10 \%\end{array}$ & 505 (46.5\%) & & - & - & $\begin{array}{l}\text { Meeting guidelines } \\
\text { Boys: } 38 \% \\
\text { Girls: } 25 \%\end{array}$ & - \\
\hline $\begin{array}{l}\text { United } \\
\text { Kingdom }\end{array}$ & $\begin{array}{l}63.4 \mathrm{~min} / \text { day } \\
9,982 \text { steps/day } \\
\text { Meeting guidelines } \\
\text { Boys: } 19 \% \\
\text { Girls: } 11 \%\end{array}$ & 479 (44.1\%) & $\begin{array}{l}\text { MCS } \\
\text { (2008-2009) }\end{array}$ & $7-8$ years & $6,497(49 \%)$ & $\begin{array}{l}60.1 \mathrm{~min} / \text { day } \\
10229 \text { steps/dayd } \\
\text { Meeting guidelines } \\
\text { Boys: } 28 \% \\
\text { Girls: } 15 \%\end{array}$ & $\begin{array}{l}\text { MVPA: } \\
>2241 \\
\text { counts/min }\end{array}$ \\
\hline Portugal 10 & $\begin{array}{l}56.4 \mathrm{~min} / \text { day } \\
9,633 \text { steps/day } \\
\text { Meeting guidelines } \\
\text { Boys: } 9 \% \\
\text { Girls: } 4 \%\end{array}$ & $686(44.5 \%)$ & - & $10-11$ years & $2,714(45.0 \%)$ & $\begin{array}{l}\text { Boys: } 65.9 \mathrm{~min} / \text { day } \\
\text { Girls: } 45.3 \mathrm{~min} / \text { day } \\
\text { Meeting guidelines } \\
\text { Boys: } 51.6 \% \\
\text { Girls: } 22.5 \% \\
\end{array}$ & Trost \\
\hline \multicolumn{8}{|l|}{ Africa } \\
\hline Kenya & $\begin{array}{l}71.6 \mathrm{~min} / \text { day } \\
\text { 11,034 steps/day } \\
\text { Meeting guidelines } \\
\text { Boys: } 12.6 \% \\
\text { Girls: } 10.3 \% \\
\end{array}$ & $502(46.5 \%)$ & - & - & - & - & - \\
\hline South Africa & $\begin{array}{l}65.1 \mathrm{~min} / \text { day } \\
11,281 \text { steps/day } \\
\text { Meeting guidelines } \\
\text { Boys: } 28.9 \% \\
\text { Girls: } 24.7 \%\end{array}$ & 468 (39.3\%) & - & - & - & - & - \\
\hline \multicolumn{8}{|l|}{ The Americas } \\
\hline Canada ${ }^{11}$ & $\begin{array}{l}58.7 \mathrm{~min} / \mathrm{day}{ }^{\mathrm{e}} \\
9,132 \mathrm{steps} / \mathrm{day}\end{array}$ & $524(41.6 \%)$ & $\begin{array}{l}\text { CHMS } \\
(2007-2009)^{* *}\end{array}$ & $6-11$ years & $878(51.2 \%)$ & $63.3 \mathrm{~min} / \mathrm{day}$ & Actical $^{12}$ \\
\hline
\end{tabular}




\begin{tabular}{|c|c|c|c|c|c|c|c|}
\hline & $\begin{array}{l}\text { Meeting guidelines } \\
\text { Boys: } 26 \% \\
\text { Girls: } 18 \%\end{array}$ & & & & & $\begin{array}{l}\text { Boys: } 13217 \text { steps/day } \\
\text { Girls: } 11745 \text { steps/day } \\
\text { Meeting guidelines } \\
\text { Boys: } 31 \% \\
\text { Girls: } 21 \%\end{array}$ & \\
\hline United States $^{13}$ & $\begin{array}{l}49.8 \mathrm{~min} / \text { day } \\
8,568 \text { steps/day } \\
\\
\text { Meeting guidelines } \\
\text { Boys: } 36 \% \\
\text { Girls: } 27 \%\end{array}$ & 491 (41.3\%) & $\begin{array}{l}\text { NHANES } \\
(2003-2004)\end{array}$ & $6-11$ years & $597(51.8 \%)$ & $\begin{array}{l}\text { Boys: } 95.4 \mathrm{~min} / \text { day } \\
\text { Girls: } 75.2 \mathrm{~min} / \text { day } \\
\text { Boys: } 13000 \text { steps/day } \\
\text { Girls: } 12000 \text { steps/day } \\
\text { Meeting guidelines } \\
\text { Boys: } 30 \% \\
\text { Girls: } 24 \%\end{array}$ & Freedson \\
\hline Colombia & $\begin{array}{l}68.1 \mathrm{~min} / \text { day } \\
10,724 \text { steps/day } \\
\text { Meeting guidelines } \\
\text { Boys: } 16.7 \% \\
\text { Girls: } 7.3 \% \\
\end{array}$ & $857(49.2 \%)$ & - & - & - & - & - \\
\hline Brazil & $\begin{array}{l}59.5 \mathrm{~min} / \text { day } \\
8,721 \text { steps/day } \\
\\
\text { Meeting guidelines: } \\
\text { Boys: } 18.7 \% \\
\text { Girls: } 11.3 \% \\
\end{array}$ & 495 (49.1\%) & - & - & - & - & - \\
\hline \multicolumn{8}{|l|}{ South Asia } \\
\hline India & $\begin{array}{l}49.0 \mathrm{~min} / \text { day } \\
9,288 \text { steps/day } \\
\text { Meeting guidelines } \\
\text { Boys: } 35.6 \% \\
\text { Girls: } 22.0 \%\end{array}$ & $553(45.9 \%)$ & - & - & - & - & - \\
\hline \multicolumn{8}{|l|}{ Western Pacific } \\
\hline China & $\begin{array}{l}45.2 \mathrm{~min} / \text { day } \\
2,664 \text { steps/day } \\
\text { Meeting guidelines } \\
\text { Boys: } 12.3 \% \\
\text { Girls: } 10.8 \% \\
\end{array}$ & $502(52.0 \%)$ & - & - & - & - & - \\
\hline Australia $1,14,15$ & $64.8 \mathrm{~min} / \mathrm{day}$ & 491 (45.8\%) & ANCNPAS & 9-11 years & 530 & 12230 steps/day & - \\
\hline
\end{tabular}




\begin{tabular}{|l|l|l|l|l|l|l|}
\hline & $\begin{array}{l}\text { 10,262 steps/day } \\
\text { Meeting guidelines }\end{array}$ & & $(2007)$ & & \\
& Boys: $36.3 \%$ & & & & \\
Girls: $25.7 \%$ & & & & \\
\hline
\end{tabular}

aUnless otherwise noted, Evenson cut-points were used for ISCOLE accelerometer data (i.e., sedentary: 0-25 counts/15 seconds; light: $26-573$ counts/15 seconds;

moderate-to-vigorous: $\geq 574$ counts $/ 15$ seconds ${ }^{16}$ ). ${ }^{\text {PP }}$ Proportion of children meeting physical activity guidelines was obtained via child-report questionnaire. ${ }^{c}$ Proportion

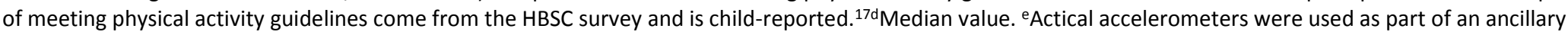
study in ISCOLE Canada so that data could be compared to that from the CHMS.

ANCNPAS: Australian National Children's Nutrition and Physical Activity Survey; CHMS: Canadian Health Measures Survey; MCS: Millennium Cohort Study; NHANES: National Health and Nutrition Examination Survey. 
Supplementary file 5: Mean screen time, and proportion of children meeting screen time guidelines in ISCOLE sites and other national datasets

\begin{tabular}{|c|c|c|c|c|c|c|}
\hline \multirow[t]{2}{*}{ Study site } & \multicolumn{2}{|l|}{ ISCOLE sample } & \multicolumn{4}{|c|}{ National sample } \\
\hline & $\begin{array}{l}\text { Screen time }{ }^{a} \text { (hr/day, } \\
\text { SD) }\end{array}$ & n (\% males) & Dataset & Age & $\mathrm{n}$ (\% males) & Screen time (mean $\mathrm{hr} /$ day, SD) ${ }^{\mathrm{b}}$ \\
\hline \multicolumn{7}{|l|}{ Europe } \\
\hline Finland & $\begin{array}{l}\text { Weekday: } 2.7(1.5) \\
\text { Weekend: } 3.7(2.0)\end{array}$ & 536 & - & - & - & - \\
\hline United Kingdom $^{18}$ & $\begin{array}{l}\text { Weekday: } 2.6(1.8) \\
\text { Weekend: } 3.8(2.2)\end{array}$ & 525 (45.1\% male) & HBSC (2010) & $\begin{array}{l}11,13 \text { and } 15 \\
\text { year olds }\end{array}$ & $\begin{array}{l}\mathrm{N}=4,404 \\
\text { Sample size with } \\
\text { information } \\
\text { available on } \\
\text { gender: } 4,383 \\
\text { (43\% boys) }\end{array}$ & $\begin{array}{l}\text { Proportion of participants watching }>2 \\
\text { hours TV/DVD per day (weekday): } \\
\text { Boys age 11: } 63 \% \\
\text { Girls age 11: } 59 \% \\
\text { Proportion of participants that play >2 } \\
\text { hours of computer games (weekday): } \\
\text { Boys age } 11: 52 \% \\
\text { Girls age } 11: 24 \%\end{array}$ \\
\hline Portugal19 & $\begin{array}{l}\text { Weekday: } 2.1(1.4) \\
\text { Weekend: } 3.6(2.1)\end{array}$ & 777 & HSBC (2010) & $\begin{array}{l}11,13 \text { and } 15 \\
\text { years old }\end{array}$ & $5,050(47.7 \%)$ & $\begin{array}{l}\text { Proportion of children watching } \geq 1 \text { hour } \\
\text { TV } \\
\text { Weekday: } \\
\text { Total: } 85.9 \% \\
\text { Boys: } 86.5 \% \\
\text { Girls: } 85.4 \% \\
\text { Weekend: } \\
\text { Total: } 94.74 \% \\
\text { Boys: } 94.6 \% \\
\text { Girls: } 95.0 \% \\
\text { Proportion of children using the } \\
\text { computer } \geq 1 \text { hour } \\
\text { Weekday: } \\
\text { Total: } 70.7 \% \\
\text { Boys: } 71.8 \% \\
\text { Girls: } 69.7 \% \\
\text { Weekend: } \\
\text { Total: } 81.4 \% \\
\text { Boys: } 80.8 \% \\
\text { Girls: } 82.0 \%\end{array}$ \\
\hline \multicolumn{7}{|l|}{ Africa } \\
\hline Kenya & Weekday: 1.9 (1.9) & 562 & - & - & - & - \\
\hline
\end{tabular}




\begin{tabular}{|c|c|c|c|c|c|c|}
\hline & Weekend $4.4(2.5)$ & & & & & \\
\hline South Africa & $\begin{array}{l}\text { Weekday: } 2.8(2.2) \\
\text { Weekend: } 4.7(2.7)\end{array}$ & 541 & - & - & - & - \\
\hline \multicolumn{7}{|l|}{ The Americas } \\
\hline Canada & $\begin{array}{l}\text { Weekday: } 2.4(1.8) \\
\text { Weekend: } 3.6(2.2) \\
\text { Total: } 2.8(1.8)\end{array}$ & 567 & $\begin{array}{l}\text { CHMS (2006- } \\
2007)\end{array}$ & $6-11$ & $878(51.2 \%)$ & Total: 2.5 (mean, $95 \% \mathrm{Cl}: 2.4-2.7)$ \\
\hline United States 20 & $\begin{array}{l}\text { TV hours per day: } \\
\text { Mean }(95 \% \mathrm{Cl}) \\
\text { Boys: } 2.2(2.0-2.3) \\
\text { Girls: } 2.2(2.0-2.3) \\
\text { Computer hr/d: Mean } \\
\underline{(95 \% \mathrm{Cl})} \\
\text { Boys: } 1.8(1.60-1.9) \\
\text { Girls: } 1.2(1.1-1.3) \\
\end{array}$ & $634(43.21 \%)$ & $\begin{array}{l}\text { NHANES (2001- } \\
\text { 2004) }\end{array}$ & $\begin{array}{l}9-11 \\
\text { Mean age in } \\
\text { months: } 125.5\end{array}$ & $1,078(52.3 \%)$ & $\begin{array}{l}\text { TV hours per day: Mean }(95 \% \mathrm{Cl}) \\
\text { Boys: } 2.4(2.2-2.6) \\
\text { Girls: } 2.4(2.2-2.5) \\
\text { Computer hr/d: Mean }(95 \% \mathrm{Cl}) \\
\text { Boys: } 1.2(1.0-1.4) \\
\text { Girls: } 0.8(0.7-0.9)\end{array}$ \\
\hline Colombia & $\begin{array}{l}\text { Weekday: } 2.8(1.6) \\
\text { Weekend: } 3.6(2.0)\end{array}$ & 919 & ENSIN 2010 & $5-12$ years & $18,527(51.5 \%)$ & $2.4 \mathrm{hr} / \mathrm{day}$ \\
\hline Brazil & $\begin{array}{l}\text { Weekday: } 3.5(2.4) \\
\text { Weekend: } 4.9(2.6)\end{array}$ & 569 & - & - & - & - \\
\hline \multicolumn{7}{|l|}{ South Asia } \\
\hline India & $\begin{array}{l}\text { Weekday: } 1.7(1.2) \\
\text { Weekend: } 3.0(1.8)\end{array}$ & $620(47.1 \%)$ & - & - & - & - \\
\hline \multicolumn{7}{|l|}{ Western Pacific } \\
\hline China & $\begin{array}{l}\text { Weekday: } 1.9(1.7) \\
\text { Weekend: } 3.0(2.2) \\
\text { Total: } 2.2(1.6) \\
\end{array}$ & 550 & CHNS (1997) & $6-18$ years & $2702(53.3 \%)$ & $\begin{array}{l}\text { Median (IQR) min/wk } \\
\text { Males: } 420(180-600) \\
\text { Females: } 420(180-600)\end{array}$ \\
\hline Australia & $\begin{array}{l}\text { Weekday: } 2.6(1.7) \\
\text { Weekend: } 4.0(2.3) \\
\text { Total: } 2.9(1.7)\end{array}$ & 527 & ANCNPAS (2007) & $9-11$ years & 538 & 203 (102) $\mathrm{min} /$ day \\
\hline
\end{tabular}

aChild-reported screen time was determined from a Diet and Lifestyle Questionnaire. ${ }^{21}$ Children were asked how many hours they typically watched TV, and how many hours they played video games and/or used the computer per week day, and per weekend day. Responses were: $1=1$ did not watch TV, $2=$ Less than 1 hour, $3=1$ hour, $4=2$ hours, $5=3$ hours, $6=4$ hours, $7=5$ or more hours. Response categories were collapsed to include $0-1$ hour of TV, 2 hours, 3 hours, 4 hours, and 5 hours or more. A weighted mean score of hours of screen time per week was then calculated as follows: [(hours of TV on weekdays $\times 5)+($ hours of TV on weekend days $\times 2$ ) $+($ hours of video games and computers on weekdays $\times 5)+$ (hours of video games and computers on weekend days $\times 2$ )]/7. For analysis, this is presented as a screen time score, rather than total hours of ST since after 5 hours per day, we could not ascertain the participant's actual amount of ST. bUnless otherwise reported, screen time was a composite measure of all television, video game, and computer use during leisure time

ANCNPAS: Australian National Children's Nutrition and Physical Activity Survey; CHMS: Canadian Health Measures Survey; CHNS: China Health and Nutrition Survey; ENSIN: Encuesta Nacional de la Situación Nutricional (National Survey of Nutritional Status); HBSC: Health Behaviors in School-aged Children; NHANES: National Health and Nutrition Examination Survey. 


\section{References}

1 Department of Health and Ageing. Australian National Children's Nutrition and Physical Activity Survey (ANCNPAS): main findings. Commonwealth Scientific and Industrial Research Organisation (CSIRO) and the University of South Australia: Canberra, Australia, 2008.

2 Tremblay M, Wolfson M, Connor Gorber S. Canadian Health Measures Survey: rationale, background and overview. Health Rep 2006; 18 Suppl: 7-20.

3 Giroux S. Canadian Health Measures Survey: sampling strategy overview. Health Rep 2006; 18 Suppl: 31-36.

4 Sardinha LB, Santos R, Vale S, Silva AM, Ferreira JP, Raimundo AM et al. Prevalence of overweight and obesity among Portuguese youth: a study in a representative sample of 10-18-year-old children and adolescents. Int J Pediatr Obes 2011; 6: e124-128.

5 Tremblay M, Shields M, Laviolette M, Craig C, Janssen I, Connor Gorber S. Fitness of Canadian children and youth: results from the 2007-2009 Canadian Health Measures Survey. Health Rep 2010; 21: 7-20.

6 Ogden CL, Carroll MD, Kit BK, Flegal KM. PRevalence of childhood and adult obesity in the united states, 2011-2012. JAMA 2014; 311: 806-814.

7 Chinese Students' Constitution and Health Research Group. Reports on the Physical Fitness and Helath Research of Chinese School-aged Students in 2010. China High Educ Press 2011. 
8 Lifestyle Statistics Team. National Child Measurement Programme: England, 2012/2013 school year. Health and Social Care Information Centre, Public Health England, 2013.

9 Griffiths L, Cortina-Borja M, Sera F, Pouliou T, Geraci M, Rich C et al. How active are our children? Findings from the Millennium Cohort Study. BMJ Open 2013; 3:

e002893.

10 Baptista F, Santos DA, Silva AM, Mota J, Santos R, Vale S et al. Prevalence of the Portuguese Population Attaining Sufficient Physical Activity: Med Sci Sports Exerc 2012;

44: $466-473$.

11 Colley RC, Garriguet D, Janssen I, Craig CL, Clarke J, Tremblay MS. Physical activity of Canadian children and youth: Accelerometer results from the 2007 to 2009 Canadian Health Measures Survey. Health Rep 2011; 22: 15-23.

12 Colley RC, Tremblay MS. Moderate and vigorous physical activity intensity cutpoints for the Actical accelerometer. J Sports Sci 2011; 29: 783-789.

13 Troiano RP, Berrigan D, Dodd KW, Mâsse LC, Tilert T, McDowell M. Physical activity in the United States measured by accelerometer. Med Sci Sports Exerc 2008; 40: $181-188$.

14 Australian Bureau of Statistics. Australian Health Survey: Physical Activity, 201112.

2013.http://www.abs.gov.au/ausstats/abs@.nsf/Lookup/4364.0.55.001main+features1 2011-12 (accessed 10 Oct2014). 
15 Australian Bureau of Statistics. Census of Population and Housing: SocioEconomic Indexes for Areas (SEIFA).

2011.http://www.abs.gov.au/ausstats/abs@.nsf/Lookup/2033.0.55.001main+features1 00132011 (accessed 10 Oct2014).

16 Evenson KR, Catellier DJ, Gill K, Ondrak KS, McMurray RG. Calibration of two objective measures of physical activity for children. J Sports Sci 2008; 26: 1557-1565.

17 Currie C, Zanottie C, Morgan A, Currie D, de Looze M, Roberts C et al. Social determinants of health and well-being among young people. World Health Organization: Copenhagen, WHO Regional Office for Europe, 2012.

18 Currie C, Gabhainn SN, Godeau E, Roberts RS, Currie O, Picket W et al. Inequalities in Young People's Health: Health Behaviour in School-aged Children. International Report from the 20052006 Survey Health Policy for Children and Adolescents. 2008.

19 Matos M, Simões C, Tomé G, Camacho I, Ferreira M, Ramiro L et al. A saúde dos adolescents portugueses - Relatório do estudo HSBC 2010. Aventura Socia \& Saúde: Portugal, 2010.

20 Anderson SE, Economos CD, Must A. Active play and screen time in US children aged 4 to 11 years in relation to sociodemographic and weight status characteristics: a nationally representative cross-sectional analysis. BMC Public Health 2008; 8: 366.

21 Katzmarzyk PT, Barreira TV, Broyles ST, Champagne CM, Chaput J-P, Fogelholm M et al. The International Study of Childhood Obesity, Lifestyle and the Environment (ISCOLE): design and methods. BMC Public Health 2013; 13: 900. 


\section{Appendix B \\ Background Manuscript 2}

Knowledge and awareness of Canadian Physical Activity and Sedentary Behaviour Guidelines: a synthesis of existing evidence.

This manuscript has been written and formatted to adhere to the specifications for submission to the journal Applied Physiology, Nutrition, and Metabolism. This manuscript is currently in press.

(http://www.nrcresearchpress.com/doi/abs/10.1139/apnm-2014-0464) 


\title{
Knowledge and awareness of Canadian Physical Activity and Sedentary Behaviour Guidelines: a synthesis of existing evidence
}

\author{
Allana G. LeBlanc, Tanya Berry, Sameer Deshpande, Mary Duggan, Guy Faulkner, \\ Amy E. Latimer-Cheung, Norm O'Reilly, Ryan E. Rhodes, John C. Spence, and Mark S. Tremblay
}

\begin{abstract}
The aim of this review was to consolidate and synthesize existing evidence regarding current knowledge and awareness of the Canadian Physical Activity (PA) and Sedentary Behaviour (SB) Guidelines. MFDLINE, Embase, and PsyclNFo were searched for peer-reviewed publications pertaining to the guidelines. Content experts, key organizations (i.e., ParticipACTION and the Canadian Fitness and Lifestyle Research Institute), journal Web sites, and service organizations (i.e., the Canadian Society for Exercise Physiology (CSEP) and the Public Health Agency of Canada) were consulted for additional evidence. Scientific publications $(n=6)$ and research from Participacrion and the Canadian Fitness and lifestyle Research Institute reported that awareness of the guidelines is low, especially with respect to the SB guidelines. Less than 105 of survey respondents from the Canadian population were aware of the PA guidelines, and less than $5 \%$ were aware of the SB guidelines. Information on the guidelines was available on 51X of public health unit and CSEP partner Web sites. Online metrics (e.g., downloads, site accessions from CSEP, the Public Health Agency of Canada, and journal Web sites showed that online accession of the guidelines was high (e.g., all "highly accessed" on journal Web sites). This review showed that awareness of the Canadian PA and SB Guidelines is low among the general population but higher among the scientific and stakeholder communities. Governmental, nongovernmental, and stakeholder organizations should collabonate in creating sustained, long-term, and well-resourced communication plans to reach the Canadian population to raise awareness of $\mathrm{PA}$ and SB guidelines and should implement programs to facilitate their uptake.
\end{abstract}

Key wards: guidelines, public health, communications, review.

Résumé : L'objectif de cette analyse documentaire est de consolider et de synthétiser les données probantes quant à la connaissance courante et la sensibilisation aux Directives canadiennes en matière d’activité physique (PA) et de comportement sédentaire (SB). On a consulté dans MEDLINE, Embase et PsycINFo les articles sanctionnés par les pairs ayant trait aux directives. Pour obtenir d'autres données probantes, on a consulté des experts en la matiëre, des organisations clés (c-ì-d. ParticipACTnON, Institut canadien de la recherche sur la condition physique et le mode de vie), les sites Internet des périodiques et organismes de services (c-ä-d. Société canadienne de physiologie de lexercice (SCFE) et l'Agence de santé publique du Canada. Les publications scientifiques $(n=6)$ et les études réalisées par ParticipMCTION et l'Tnstitut canadien de la recherche sur la condition physique et le mode de vie révélent que la sensibalisation aux directives est faible surtout en ce qui concerne les directives en matière de comportement sédentaire. Moins de $10 \%$ des répondants au questionnaire dans la population canadienne sont sensibilisés aux directives en matière d’activité physique et moins de $5 \%$ sont sensibilisés aux directives en matière de comportement sédentaire. Les renseignements au sujet des directives sont disponibles sur les sites Internet de la SCPE et de $51 \%$ des bureaux de santé publique. Le dénombrement des accès en ligne (p. ex. téléchargements, visites du site) i la SCPE, lAggence de santé publique du Canada et aux périodiques en ligne indiquent que l'accès aux directives en ligne est elevé (p. ex. tous s très fréquentés s) sur les sites des périodiques). Cette analyse documentaire montre que la sensibilisation aux directives en matière d'activité physique et de comportement sédentaire est faible au sein de la population globale, mais élevée dans la communauté scientifique et le milieu des intervenants. Les organisations gouvernementales, non gouvernementales et les organismes dintervenants devraient collaborer à lélaboration de plans pour une communication soutenue, bien documentée et à long terme afin de joindre la population canadienne et d’accroitre sa sensibilisation aux directives en matière d'activité physique et de comportement sédentaire et de mettre en cuvve des programmes facilitant leur adoption. [Traduit par la Rédaction]

Mots-clés : directives, santé publique, comumunication, analyse documentaire.

Received 28 October 2014. Accepted 29 junuary 2015.

A.G. LeBlanc and M.S. Tremblay. Healthy Active Living and Obesity Research Group, Children's Hospital of Eastern Ontario Research Institute,

401 Smyth Road, Ottawa, ON K1H 8L1, Canada; Fopulation Health, Faculty of Graduate and Fostdoctoral Studies, University of Ottawa, Ottawn,

ON KIN 6N5, Canada.

T. Berry and J.C. Spence. Faculty of Physical Education and Recreation, University of Alberta, Edmonton, AB T6C 2H9, Canada

S. Deshpande. Faculty of Management, University of Lethbridge, Lethbridge, AB TIK 3M4, Canada.

M. Duggan. Canadian Society for Exercise Physiology, Ottawa, ON K2P of2, Canada.

G. Faullener. Faculty of Kinesiology and Physical Education, University of Toronto, Toronto, ON Mss 2W6, Canada.

A.E. Latimer-Cheung. School of Kinesiology and Health Studies, Queen's University, Kingston, ON K7L 3N6, Canada.

N. O'Reilly. Department of Sports Administration, Ohio University, Athens, OH 45701, USA.

R.E. Rhodes. Behavioural Medicine Laboratory, University of Victoria, Victoria, BC V8P 5C2, Canada.

Corresponding author: Allana G. LeBlanc (e-mail: alleblancefecheo.on.ca). 


\section{Introduction}

The Canadian Physical Activity (PA) Guidelines have sought to provide health professionals, researchers, and the public with evidenceinformed targets to follow for health benefits and have the potential to contribute significantly to improvements in overall health across Canada (Crimshaw and kussell 1993; Tremblay et al. 2007a, 2011c). Health Canada and the Canadian Society for Exercise Physiology (CSEP) released Canada's first PA recommendations for adults in 1998 (Health Canada and the Canadian Society for Exercise Physiology 1998), for older adults in 1999 (Health Canada and the Camadian Society for Exercise Physiology 1999), and for children and youth in 2002 (Health Canada and the Canadian Society for Exercise Physiology 2002a, 2002b). In 2006, CSEP initiated a process to update the guidelines. In 2011, in partnership with the Healthy Active Living and Obesity Research Group (www.haloresearch.ca) and ParticipACTHON (www.participhCTON.com), CSEP released evidence-informed PA guidelines for children (aged 5-11 years) youth (aged 12-17 years), adults (aged 18-64 years), and older adults (aged $\geq 65$ years) (Tremblay et al. 2011c). Sedentary behaviour (SB) guidelines were also released for children (aged 5-11 years) and youth (aged 1217 years) (Tremblay et al. 2011a). The following year, PA and SB guidelines for the early years (ages 0-4 years) were released (Tremblay et al. 2012a, 2012b). Table 1 provides a brief outline of the guidelines and their associated health benefits.

The initial Canadian PA Guidelines were disseminated in 2 steps. First was a media launch to raise awareness of low levels of PA and to direct people to the appropriate Web sites for information. The second step involved dissemination of the guides through intermediaries and professional groups involved in PA promotion. Dissemination strategies for the current PA and SB guidelines followed a similar plan, with an initial media release followed by promotion through researchers, health-care providers, governmental and nongovernmental organizations, teachers, and those with an interest in healthy active living. To date, the primary dissemination channel for Canadian guidelines has been through mass media campaigns induding print and television and radio campaigns and Web sites.

In 2007 , shortly after initiating the process of updating the guidelines, a baseline assessment of awareness and uptake was conducted by the Canadian Fitness and Lifestyle Research institute (CFI.RI) using the Physical Activity Monitor (PAM) survey (Cameron et al. 2007). The PAM provides a nationally representative ongoing metric of PA among Canadians. At that time, only $3.9 \%$ of adults provided correct and unprompted recall of the PA guidelines. Prompted recall of the guidelines was much higher (37\%), suggesting the possibility of a social desirability bias. slightly lower levels of prompted recall (21:5) have been shown elsewhere, with only $6 \%$ of survey respondents stating that they actually follow the recommendations (Spence et al. 2002). Low levels of guideline awareness are not limited to Canadians, as a recent study showed, only $36.1 \%$ of Americans were aware of US PA guidelines and only $0.56 \%$ of respondents were able to correctly identify the guidelines with prompted questioning (Kay et al. 2014).

A systematic review led by the Centers for Disease Control Task Force showed insufficient evidence to determine the effectiveness of stand-alone mass media campaigns for increasing PA (Brown et al. 2012). For successful knowledge dissemination, the task force suggests that media campaigns be integrated into a broader, multicomponent, community-wide intervention (Brown et al. 2012). Since the new Canadian guidelines were released, little work has been done to gauge their awareness among Canadians, and therefore it is unknown whether current efforts to disseminate the new guidelines have been effective, or if more intensive population health interventions are required. The purpose of this review was to consolidate and synthesize the available evidence to understand the current knowledge and awareness of the Canadian PA and SB Cuidelines. This synthesis can be used to inform future knowledge translation strategies and materials for PA and SB guidelines in Canada and elsewhere.

\section{Materials and methods}

This project required gathering and synthesizing data from many different sources including ParticipACHON, CFLRI, CSEP, the Public Health Agency of Canada (PHAC), scientific databases, and recognized content experts.

\section{ParticipACTION}

ParticipacmoN has focused many of its media and outreach campaigns on awareness, understanding, and uptake of the Canadian PA Guidelines (ParticipACTION 2014). The "Bring Back Play" campaign, targeted to mothers of children aged 5-11 years, aired from October 2012 to March 2014 with the aim of getting children outdoors to experience the fun and benefits of unstructured play. Immediately following the campaign, ParticipACTION commissioned a survey of randomly selected participants representative of the general population, and of mothers of children aged 5-11 years. Respondents were asked 21 questions related to the campaign (e.g., "Can you name any organizations that promote physical activity? ) and guideline awareness (e.g., "Have you heard of the Canadian PA Guidelines for Children and Youth announced January $\left.20112^{\circ}\right)$. The survey included both prompted and unprompted questions. Respondents were recruited from an online Angus Reid Forum of more than 125000 Canadians. The final survey included 770 respondents from the general population (weighted by sex, age, and region), and 1046 mothers (weighted by region) (unpublished ParticipACTON campaign report data, 2015).

\section{CFIRI}

Data from the CFLRI came from the PAM. The PAM is a series of nationally representative telephone surveys that was established in 1995 as a tool to help policy makers measure progress in changing relevant individual, environmental, and societal factors in an effort to decrease physical inactivity. The PAM includes unprompted and prompted questions about respondents' awareness of PA guidelines (e.g., "Have you heard, or do you know of any guidelines about how much physical activity adults should dor) The 2013-2014 PAM will yield a sample size of roughly 8000 adults over 18 years of age; interim results, based on data available to date, are presented here (unpublished interim CFLRI PAM data, 2014) $(n=2765)$ ).

\section{CSEP online metrics}

CSEP has dedicated a section of its Web site to information and documents (in English and French) related to the PA and SB guidelines (www.csep.calguidelines). Individuals can also order paper copies of the guidelines and/or the guideline handbook (as pads of 50 copies or as a collection of all the guidelines and the handbook). Organizations in 2 provinces (i.e., Service Ontario and Healthy Families BC) have developed a partnership with CSEP to provide paper copies of the guidelines free of charge to all provincial residents through requests on the CSEP Web site; residents of other provinces are required to pay for hard copies but can download the PDF free of charge. As part of the media release associated with the guidelines, CSEP also calculated media impressions (i.e, the potential number of people who may have heard of the guidelines via television, print, or Internet media coverage). Web site accessions were captured primarily via Coogle analytics; purchases of the guidelines and/or handbook were measured through the CSEP online store.

\section{PHAC online metrics}

PHAC played an important role in the development and initial dissemination of the PA guidelines and also has a Web page dedicated to the Canadian PA Guidelines, with information available in both English and French (www.phac-aspc.gc.ca/hp-ps! hl-mvs/pa-ap/index-eng php). PHAC's Web site includes tip sheets, information on the benefits of being active, background information, and a link to the guidelines via the CSEP Web site. PHAC does not have information or links related to the PA guidelines for the 


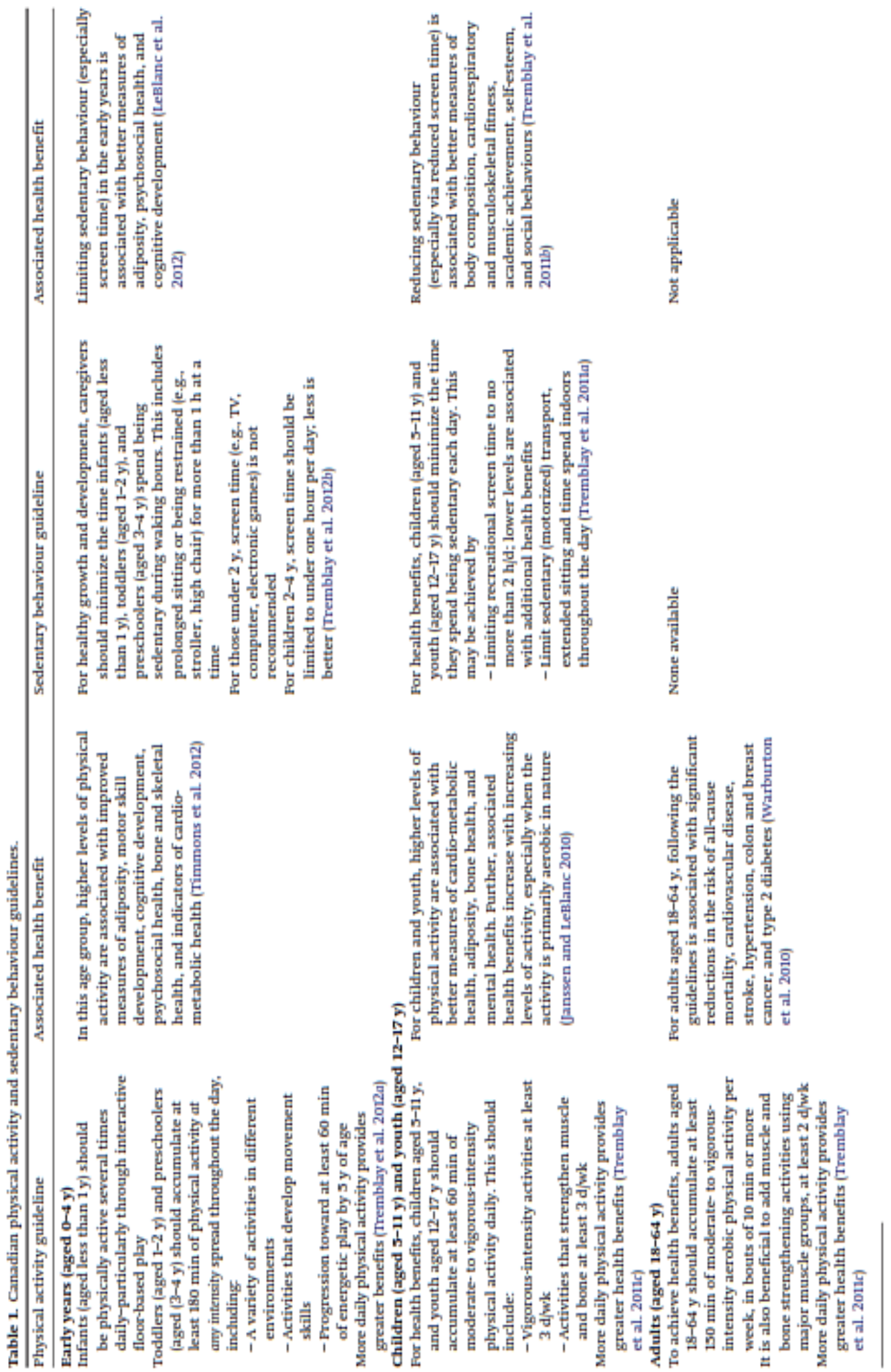

t Published by NRC Research Press 
early years (aged 0-4 years) or the SB guidelines for any age group on their Web site.

\section{Scientific literature}

MPDLINF, Embase, and PsyciNFo were searched to identify peerreviewed publications pertaining to the new Canadian guidelines. subject headings most similar to "guidelines", AND "physical activity" OR "sedentary", and limited to the past 3 years (i.e., since 2011 when the new guidelines were released) were used (see Appendix A for complete search strategies). To be included, studies needed to assess awareness or uptake of the new Canadian PA or SB Guidelines.

In addition, the ParticipACrion Research Advisory Group (paper authors), a group composed of 8 researchers from across Canada with expertise ranging from PA assessment to strategies for effective health-related behaviour-change strategies, were asked to identify any additional research pertaining to Canadian guideline awareness. CSEP and ParticipACTION also sent out a query in their respective newsletters asking for any additional research relating to the guidelines. In total, this request was sent to more than 10000 CSEP and ParticipACTON network members across Canada, including health-care providers, fitness professionals, researchers, and others working in the health and fitness fields.

\section{Results \\ ParticipACTION}

With un prompted questioning, respondents, on average, reported that the appropriate frequency for children to be physically active is for at least $60 \mathrm{~min}, 6$ days per week. Although guidelines state that children and youth should engage in 60 min of moderate- to vigorous-intensity PA every day, 6 out of 7 days is often how the guidelines are operntionalized in surveillance studies (Colley et al. 2011). When asked if they had heard of the Canadian PA Guidelines, $8 \%$ of the general public and $12 \%$ of mothers responded that they had heard of the Canadian PA Guidelines for children and youth; this has decreased from $14 \%$ (for both groups) after the guidelines were released in 2011. When prompted, 335 of the general population and 40x of mothers identified the main component of the PA guidelines as being the recommendation that children and youth should accumulate 60 min of moderate- to vigorousintensity PA daily. Awareness of the SB guidelines is lower than awareness of the PA guidelines, with $35 \%$ of the general public and $3 \%$ of mothers having heard of the guidelines for children and youth and $2 \%$ of the general population and $3 \%$ of mothers having heard of the guidelines for the early years. With prompted questioning, $70 \%$ of the general population and $66 \%$ of mothers could not identify any components of the SB guidelines for children and youth. The percentage of survey respondents who were aware of the PA guidelines for adults (aged 18-65 years) is lower (5\% of the general population and $6 \%$ of mothers having heard of the guidelines). Since the release of the guidelines for adults in 2011 , awareness has remained stable among mothers (at 6\%) but decreased from 125 in 2011 to $5 \%$ in 2014 among the general population.

\section{CFLRI}

with prompted questioning, $37.77 \%$ of adults indicated that they had heard of the Canadian PA Guidelines (provided as 1 option in a list of items such as Canada's Food Guide). Awareness of the guidelines declined with age $\left(x^{2}=31.3\right.$, df $\left.=3, p \leq 0.0001\right)$, with recall being highest among 18- to 24 -yearolds $(48.3 \%$, $95 \% \mathrm{Cr} 35.3-$ $61.5 x)$ and lowest among those over 45 years of age (45-64 years: $33.4 \%$, 95\% CI 29.7x-37.3\%; ¿265 years: 33.3\%, 95\% CI 29.1\%x-37.8\%). If participants said they had heard of the guidelines, they were asked to elaborate; with unprompted questioning, only $7.6 \%$ of participants responded correctly. Unprompted recall differed by $\operatorname{sex}\left(x^{2}=12.6, \mathrm{df}=1, p \leq 0.0001\right)$ and age group $\left(x^{2}=46.7, \mathrm{df}=3, \mathrm{p} \leq\right.$ $0.0001)$. More women $(9.3 \%, 95 \% \mathrm{CI} 7.4 \times-11.6 \%)$ than men (5.7\%,

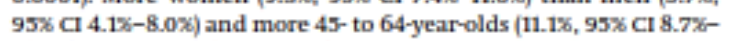
$14.0 \%)$ than adults 265 years $(5.0 \%, 95 \% \mathrm{Cl} 3.5 \%-7.1 \%)$ were able to give a correct response. 


\section{CSEP online metrics}

From February 2011 to June 2014, more than $\$ 80000$ worth of guideline handbooks and pads were distributed. of this, 1238 (20.0\%) French) pads of PA guidelines were distributed for children in the early years, 4231 (13.6\% French) pads for children and youth, 2520 (13.2\% French) pads for adults, and 1901 (36.7\% French) pads for older adults. With respect to the SB guidelines, 461 (19.3\% French) pads for children in the early years and 1611 (14.2\% French) pads for children and youth were distributed. This translates to almost 600000 copies of the guideline information sheets being distributed since their release. In addition, as of july 2014, 7643 handbooks had been purchased, and the handbook had been viewed online almost 60000 times. The guidelines section is the most popular page on the CSEP Web site (after the home page), with more than 325000 views from when the first communique on the new guidelines was released in December 2010 to July 2014. The public relations company handling the media release of the guidelines calculated more than 42.5 million media impressions during their initial release.

\section{PHAC online metrics}

The monitoring period for the PHAC Web site statistics was from January 1, 2011, to July 1, 2014. The page explaining the guidelines for adults was the most popular, with more than 215000 page views (23.9\% French), followed by the page hosting the guidelines for children (124399 page views, $21.5 \times$ French) and then for youth (101 420 page views, 20.8\% French). The page hosting guideline information for older adults had considerably fewer page views ( 86892 page views, 26.2\% French). The number of guideline information sheets that were downloaded from the PHAC Web site could not be ascertained because the PHAC web site links directly to the guidelines hosted on the CSEP Web site.

\section{Scientific literature}

The initial search yielded 214 unique articles. Twenty-three articles were retained for fulltext review (see Fig. 1 for PrusMA diagram (Moher et al. 2009)|. Three articles were kept and are summarized here. One additional article was identified via content experts. The newsletter request to the CSEP and ParticipACTION networks provided no additional resources to include in the review.

Carson and colleagues (2013) examined pediatricians' awareness of, agreement with, and use of the PA and SB guidelines for the early years, children, and youth. The survey included 20 questions relating to awareness (e.g., "How familiar are you with the Canadian Physical Activity-Sedentary Behaviour Guidelines?'). knowledge (e.g., "What are the main recommendations within the Camadian Physical Activity-Sedentary Behaviour Guidelines?"), agreement (e.g., "Do you agree with these recommendations? ). and uptake (e.g., "How often do you make these recommendations?"). of the 2036 pediatricians contacted initially, 331 provided sufficient data to be included in the analysis. of the final sample, few reported they were "very familiar" with the PA guidelines (6\% for the early years and $9 \%$ for children and youth) or SB guidelines ( $5 \times$ for the early years and $5 \%$ for children and youth). After being made aware of the guidelines, almost all "strongly agreed" or "agreed" with guideline recommendations (98.5\% and 96.4\% with PA guidelines for the early years and children and youth, respectively; $96.4 \%$ and $94.3 \%$ with the $5 B$ guidelines for the early years and children and youth, respectively).

A second study by Carson and colleagues (2014) reported a qualitative examination of parental perceptions of the SB guidelines for the early years. After being prowided a formal definition of SB, parents of children under 4 years of age participated in a semistructured focus group on knowledge of, agreement with, and credibility of the guidelines. They were also asked questions regarding their ability to put the guidelines into practice and the extent of potential resources needed to increase compliance. Overall, parents expressed their support for the intent of the guidelines and considered the information presented within the guidelines to be helpful. However, there was widespread concern that the guidelines positioned all SBs as negative and diminished the positive benefits of some quiet activities (e.g., reading, colouring). Concern was also expressed regarding the environment as a barrier to reducing sedentary time, largely because of climate and safety concerns. To put the guidelines into practice, parents asked for clarification on different types of SBs and how they should monitor non-screen-based activities.

Gainforth and colleagues (2013) evaluated the uptake of PA and SB guidelines on service organization Web sites. They tracked the rate of adoption of the new guidelines on Web sites of Canadian organizations whose goal is to promote PA to the general population. The list of service organizations was taken from the CSEP directory of stakeholder organizations and public health units across Canada. The final list included 45 CSEP stakeholder groups and 114 public health units. of these organizations, $51 \%$ posted information on the new guidelines over the 9-month surveillance period; the presence of information on SB guidelines peaked at 9\%. The most common strategy to promote the guidelines was via the inclusion of a link to Web sites housing the guidelines (e.g. CSEP, ParticipACTION, PHAC). The authors of this work suggest that the relatively modest rate of Web site adoption and the overall plateau in adoption levels among the general public indicate that uptake has yet to reach the critical mass needed for successful diffusion.

Martyniuk and Tucker (2014) examined early childhood education students' awareness of the guidelines. They invited all those enrolled in an early childhood education program in the province of Ontario to participate in the study examining knowledge and training related to $\mathrm{PA}$ in young children. In total, 1113 students completed the survey. Of those who completed the survey, 91.6\% felt that PA was "somewhat important" or "very important" in their own lives, and 94.7\% felt it was "very important" for young children. With prompted questioning, it was found that 28.776 were familiar with the Canadian PA Guidelines for the early years, and $8.4 \%$ were familiar with the Canadian SB Guidelines for the early years.

Content experts also identified 2 additional unpublished studies relating to guideline awareness. The first study included an assessment of parents' prompted awareness of the Canadian PA Guidelines for children and the screen-time component of the SB guidelines ( $n=500$ parents with at least 1 child aged 5-11 years) (Jarvis 2014). After reviewing the CSEP fact sheet for the Canadian PA Guidelines for children and a modified CSEP fact sheet for the SB guidelines for children, parents were asked if they had been aware of the child PA and/or screen-time guidelines before having seen the fact sheets. Fifty-one percent of parents ( $54.4 \%$ of mothers and $41.0 \%$ of fathers) indicated that they had been aware of the child PA guidelines, and $35 \%$ ( $37.77 \%$ of mothers and $25.4 \%$ of farthers) indicated that they had been aware of the screen-time component of the SB guidelines.

The second unpublished research study is from the ongoing evaluation of the impact of ParticipACnoN funded by the Canadian Institutes of Health Research. Baseline data were collected in 2007 , before the relaunch of the new Participscrion (Plotnilkoff et al. 2009). Follow-up data were collected in February 2013 from $617 \mathrm{Ca}$. nadian organizations involved with PA promotion (G. Faulkner 2014, personal communication). Several questions were included in the follow-up survey that assessed awareness of the guidelines. Among the organizations, $80.7 \%$ were aware of the PA guidelines and $24.5 \%$ were aware of the SB guidelines. The organizations also perceived the usefulness of the guidelines (1: not at all useful, 5 : very useful) to be high for both PA (3.84 out of 5) and SB (3.91 out of 5). See Appendix $\mathrm{B}$ for the specific wording of included questions where applicable.

\section{Discussion}

To the best of our knowledge, this review is the first to provide a comprehensive synthesis of knowledge, awareness, and uptake of $\mathrm{PA}$ and SB guidelines in Canada. Overall, available evidence 
Fig. 1. Prisma flow diagram. Reasons for exclusions included guidelines from outside of Canada $(n=10)$, guidelines for special populations or chronic conditions $(n=5)$, previous physical activity guides $(n=3)$, in-school guidelines $(n=3)$, and review not addressing awareness ( $n=4)$. Many studies were excluded for multiple reasons. ", Databases included the following: MEDLNE $(n=165)$, Embase ( $n=46)$, and PycDNFO ( $n=11$ ) $\uparrow, 2$ identified articles were unpublished at the time of inclusion, 1 was identified by a content expert, and the other 3 were identified via database search.

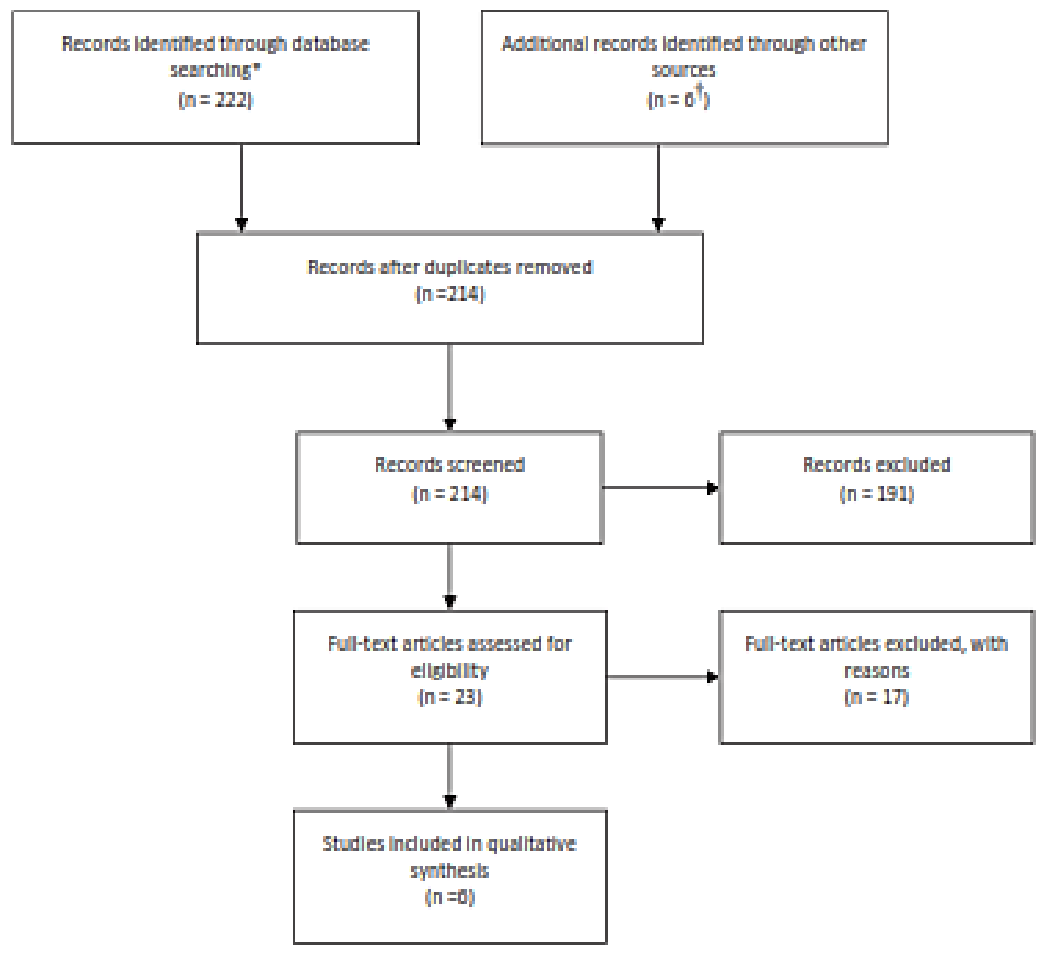

shows that guideline awareness is low among mothers, the general population, early childhood education students, and pediatricians. This is especially true for the SB guidelines. Most of the available information related to the guidelines pertains to the early years, children, and youth, and that information is gathered via parent or caregiver questionnaire. No work has assessed whether children or youth are aware of the guidelines. Little work has examined awareness or understanding of the guidelines for adults and for older adults. Demand for information on the guidelines seems to be highest for children and youth and adults, with most information being requested in English; however, it is interesting to note that there was a slightly higher demand for French information for older adults than for any other age group.

This review shows that unprompted awareness of the new guidelines, at about $4 \%$, has remained relatively unchanged from awareness of the old guidelines. This suggests that, thus far, dissemination of the guidelines has not been adequate to penetrate awareness within the general public. More encouragingly, awareness of Canadian PA Guidelines at an organizational level was high (80.75) (Gainforth et al. 2013). Puture work should aim to understand how organizations use the guidelines and to seek ways in which these organizations can help improve awareness and knowledge levels in the general population. These organizations should also be recruited to help disseminate guideline information to their respective networks.

Criticisms of the previous Canadian PA Cuidelines included a universal dislike for the cartoon-like format used to portray the information (Berry et al. 2010). It has also been suggested that the guidelines be disseminated via internet-based resources, with formatting that complements the stylistic and flashy design common in commercial, for-profit advertisements (Berry et al. 2010).
When designing the new guidelines, CSEP attempted to use a more modern look and feel; however, no research has examined whether this revamped look appeals to the general population. Internetbased learning tools should be explored as an option for guideline dissemination, especially given the requirement of continued education for many certifications (e.g., CSEP-ertified personal trainers, medical doctors). Once they have been created, online education modules require few resources or upleep and can be accessed from anywhere as long as an internet connection is available.

Overall, awareness of the PA guidelines was higher than awareness of the SB guidelines. Low levels of awareness of the SB guidelines may be, in part, a result of the terminology used. Although people may be familiar with the concept of screen time (i.e., television, video game, or computer use), they may not be familiar with the concept of SB as a distinct health risk factor (Tremblay et al. 2010a). Furthermore, influencing decisions to adopt a new behaviour (e.g., reduce SB) may require different promotion and messiging strategies than does influencing the decision to discard an old innovation (e.g., continue to follow the old PA guides). It may also be true that awareness of the PA guidelines is higher simply because they have been around for a longer time, and people have had more time to become familiar with them.

Overcoming low awareness of the guidelines will require collaboration and partnerships among those from the public health, health promotion, and academic sectors, with specific strategies to promote a "wholeday" approach to healthy lifestyle behaviours (i.e., the need to meet both PA and SB guidelines). However, it is important to remember that PA guidelines and messages alone are unlikely to curb the epidemic of inactivity. After becoming aware of the guidelines, individuals still need to have an intention to be active, to make shortterm behaviour changes through increased $\mathrm{PA}$ and decreased 
$\mathrm{SB}$, and to aim for long-term success in maintaining healthy lifestyle behaviours. Detailed population health advocacy and intervention programs with sustained support are likely to be required for this to occur.

As of June 2014, 28 peer-reviewed publications have informed or documented the development process of the Canadian PA and SB Guidelines (Brawley and Latimer 2007; Cameron et al. 2007; Esliger and Tremblay 2007; Janssen 2007; Katzmarzyk and Tremblay 2007; Martin Ginis and Hicks 2007; Paterson et al. 2007; Sharratt and Hearst 2007; Timmons et al. 2007, 2012; Tremblay et al. 2007a, 2007b, $2010 a, 2010 b$, 2011a, 2011b, 2011c, 2012a, 2012b, warburton et al. 2007 , 2010; Young and Katzmarzyk 2007; Janssen and LeBlane 2010; Kestiniemi et al. 2010; Paterson and Warburton 2010; Rhodes and Pfaeffli 2010; LeBlane et al. 2012; Latimer-Cheung et al. 2013). All these papers were published in the journal Applied Physiology, Nutrition, and Mctabolism (APNM) (impact factor 2.01 as of June 2014) or the Intematianal Journal of Bchavioural Nutrition and Fhysical Activity (IJBNPA) (impact factor $\mathbf{3 . 5 8}$ as of June 2014), and all articles are rated "highly accessed" (as defined by their respective journals standards). The article outlining the process and development of PA guidelines for all ages is the fourth most cited article in APNMs history (Tremblay et al. 2011c), whereas the original publication outlining the importance of PA in the early years is the second most accessed article in the journal's history (rimmons et al. 2007). In IJBNPA, the systematic review examining PA in children and youth is the third most accessed article in the journal's history (janssen and LeBlane 2010), whereas the systematic review examining SB in children and youth is the thirteenth most accessed in the journal's history (Tremblay et al. 2011b).

The success of peer-reviewed publications relating to the development of the guidelines should be interpreted carefully. Uptake of the scientific publications is likely driven by other researchers and probably does not reflect knowledge obtained by the general public. Readership of peer-reviewed publications by the general population is very low. However, it is encouraging that these publications are highly accessed, both in Canada and internationally. Research that informed the development of the Canadian PA and SB Guidelines has also been used to help inform PA recommendations released by the World Health Organization (WHO 2010), the United Kingdom (Bull and Expert Working Group 2010), and Australia (okely et al. 2008). Puture work should examine how often the guidelines are being used in study designs or analyses to confirm that this knowledge is getting passed on to the study participants and patients both in Canada and around the world.

The first limitation of this assessment is that it was restricted to Canadian guidelines. It is possible that papers assessing awareness and uptake of PA andjor SB guidelines from other countries would provide useful information. Such comparisons could also provide insight as to what Canada has done well and what could be improved. The large majority of the work identified in this review was focused on guidelines for those under 18 years of age, highlighting an important gap in the current literature. Future assessments should also include adults and older adults. Further, we recommend that the development of SB guidelines for adults and older adults be made a research priority, to complete guidelines for all age groups. Some of the strengths of this paper include the comprehensive and inclusive search that was conducted. Reporting on web site accessions and downloads is not often included in literature reviews, but in this case, it provided additional evidence of the potential reach of the guidelines. Further, this work included collaborations with many nongovernmental organizations whose mandate is to examine PA and SB among Canadians. Finally, this review was informed by a comprehensive series of publications related to the development and dissemination of guidelines in Canada.

\section{Conclusion}

Since their release in 2011 and 2012, little work has been done to track awareness or uptake of the Canadian PA and SB Guidelines. It is clear that the guidelines have been distributed passively through a variety of means, but available evidence shows that the vast majority of the general public is unaware of the current PA and SB guidelines, especially during unprompted questioning. Most of the work that has been conducted has focused on the guidelines for the early years, children, and youth, with little work focusing on the guidelines for adults or older adults. To augment the potential impact of future updates to the guidelines, detailed dissemination and promotion strategies with sustained support are required, and efforts should be made to assess and monitor awareness and uptake across all age (and potentially sex) subgroups. To maximize efforts, dissemination should be conducted through intersectoral partnership between federal and provincial governments as well as among nongovernmental and notforprofit groups.

\section{Acknowledgements}

We thank Jocelyn Jarvis, Christine Cameron, and Tala Chaluk Bozzer for their contributions to the information presented in this review. This review was made possible through a Mitacs ACcelerate Internship in partnership with CSEP. Competing interest: AGL. was funded by a Mitacs Accelerate Internship, in partnership with CSEP; MD is the manager of CSEP. All other authors declare that they have no competing interests. Authors' contributions: MST conceived of the idea for this manuscript. All authors helped with designing and conducting the review. AGL. drafted the manuscript. All authors critically reviewed the manuscript and provided feedback. All authors read and approved the fimal manuscript.

\section{References}

Berry, T.R., Witcher, C, Holt, N.L, and FlotniknE, R.C. 20to. A Qualitative Examination of Perceptions of Physical Activity Cuidelines and Preferences for Format. Health Promot. Pract. 11(6): 908-916. do: 10.1177$) 1524839908325066$. PMaD:19116420.

Brawley, LR., and Latimer, A. 2007. Physical activity guides for Canadians: messaging strategies, realistic expectations for change, and evaluation. Appl. messaging strategies, realistic expectations for change, and evaluation. Appl.

Erown, D.R. Soares, J., Epping, J.M., Lankford, T.J. Wallace, J.S, Hopkins, D., et al. 2012 Stand.Alone Mass Media Campaigns to Increase Physical Activity: A Community Guide Updated Beview. Am. J. Frev. Med. 43935 551-561 do: 10.1016/j.amepre.2012 07.035. FMM:23009180.

Bull, F.C, and Expert Working Group. 20t0. Fhysical activity guidelines in the U.K.: review and recommendations. School of Sport, Exrrise, and Health Sciences, Loughborough University, Leichestershire, United Kingdom.

Cameron, C., Crig, C.1, Bull, F.C., and Bauman, A. 2007. Canada's physical activity guides: has their release had an impact? Appl. Physiol. Nutr. Metab. 32/52E| 5161-5169. doi:10.1139/H07-106. FMD:18213946.

Carson, V., LeBłanc, C.M., Moerau, E., and Tremblay, M.S. 2013. Faediatricians' awareness of, agreement with and use of the new Canadian Physical Activity and Sedentary Behaviour Guidelines for children and youth zero to 17 years of age. Paediatr. Child Health 18'10\% 538-542.

Carson, $V_{-}$Clark, M., Berry, T, Holt, N., and Latimer-Cheung, A.E. 2014. A qualitative examination of the perceptions of parents on the Camadian Sedentary Behaviour Guidelines for the early years. Int. J. Behav. Nutr. Phys. Act. n1(t) 65. doi:10.1156/1479-5868-11-63.

Colley, R.C, Garriguet, D, Jussen, I., Craiz, C.1, Clarke, J and Tremblay, MS. 2011. Physical activity of Canadian children and youth:Accelerometer results
from the 2007 to 2009 Canadian Health Measures Survey. Health Rep. 22(1): from the 2007 to 2009

Esliger, D.W., and Tremblyy, MS. 2007. Physical activity and inactivity profiling: the next generation. Appl. Physiol. Nutr. Metab. 32|52 El| 5195-5207. dotio. 1139/H07-107. PMID- 12213949

Gainforth, H.L, Berry, T., Faulkner, G., Ehodes, R.E., Spence, JC, Tremblay, MS. and latimer-cheung, A.E 2013. Evaluating the uptake of Canada's new phys. ical activity and sedentary behaviour guidelines on service ormanizations ical activity and sedentary behaviour guidelines on service organizations'
websites. TransL. Behaw. Med. 322 | 172-179. doit 10.1007/s13142-012-0190-z. websites. Trans:

Grimshaw, J., and Kussell, L. 1993 . Achieving health gain through clinical guidelines. 1: Dereloping scientifically valid guidelines. Qual. Health Care, 2(4): 243-248. doi:10.1135/qshc.2.4.243. PMID:10132459.

Health Canada and the Canadian Society for Exercise Fhysiology. 1998. Canada's physical activity guide to healthy active living. Health Camada. 
Health Canada and the Canadian Society for Exercise Physiology. 1999. Canada's physical activity guide to healthy active living for older adults. Health Canadh Health Canada and the Camadian Society for Exercise Physiology, 2002a. Canada's physical activity guide for children. Health Canada.

Health Canada and the Canadian Society for Exercise Physiology. 2002b. Canadar's physical activity guide for youth Health Canada

Janssen, 1. 2007. Physical activity guidelines for children and youth. Appl. Physiol. Nutr. Metab. 32(52E): S109-5121. doi:10.1139/H0z-109. PMID:1821B942. Janssen, L., and LeBhanc, A.C. 2010. Systematic review of the health benefits of physical activity and fitness in schook aged children and youth. Int. J. Behav. Nutr. Phys Act. 7it: 40. doi-10.1186/1479-5568-7-40. PMID-20459784.

Jarvis, J.W. 2014. Investigating the message believability as a determinant of parent's intentions to support their children in meeting physical activity and screen time zuidelines. M.Sc. thesis, Queen's University, Kingston, Ontario.

Katzmarzyk, P.T., and Tremblay, M.S. 2007 . Limitations of Canada's physical activity data: implications for monitoring trends. Appl. Physiol. Nutr. Metab. 32052E: 5185-5194. doi:10.1139/H00-113. FMIID:18213948.

Kay, MC, Carroll, D.D., Carlson, S.A., and Fulton, J.E. 2014. Awareness and Knowledge of the 2008 Physical Activity Cuidelines for Americans. J. Fhys. Act. Health, 11/4: 693-698. doi:10.1123 jpah 2012-017. PMID-2349307i.

Kesaniemi. A., kiddoch, C.I., Beeder, B., Blair, S. . and Sorensen, T.L 2010. Advancing the future of ptrysical activity guidelines in Canadr: an independent expert panel interpretation of the evidence. Int J. Behav. Nutr. Fhys. Act. 7il: 4L. doi: $101186 / 2479-5868-741$. FMID:20499785.

Latimer-cheung, A.E., Rhodes, R.E, Kho, M.E., Tomasone, J.R., Gainforth, H. Kowakki, K, et al. 20B. Evidence-informed recommendations for construct ing and disseminating messages supplementing the new Canadian Physical Activity Cuidelines. BMC Public Heilth. 13/1: 419, doi:10.1156/1471-2458-1? 419. FMID:23634998.

LeBlanc, A.C, Spence, J.C, Carson, v, Gorber, S.C., Dillman, C., Janssen, 1., et al. 2012. Systematic review of sedentary behaviour and bealth indicators in the early years (aged 0-4 years). Appl. Fhysiol. Nutr. Metab. 37/4k 753-772. dot.10 I139h:2012063. FMD: 22765839,

Martin Cinis, K.A., and Hicks, A.L 2007. Considerations for the development of a physical activity guide for Canadians with physical disabilities. Appi. Physiol. Nutr. Metab. 32 52Fi: 5135-5167. doi:10 1139 H07-108. FMID:18213944.

Martyniuk, 0.j, and Tucker, P. 2014. An exploration of Early Childhood Education students' knowrledge and preparation to facilitate physical activity for preschoolers: a cross-sectional study. BMC Public Health, 14[1: 727. doE:10. 1156/1471-2455-14-727. PMID:25034415.

Moher, D. Liberati, A, Tetzlaff, J., and Altman, DC. 2009. Preferred Reporting Items for Systematic Reviews and Meta-Analyses: The PRISMA StatementThe

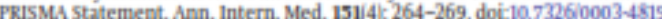
151-4.200908180-00135. PMID:19622511

Okely, A.D., Salmon, J. Trost, S.C, and Hinklky, T. 2008. Discussion paper for the development of physical activity recommendations for children under 5 years. Australian Government Department of Health and Ageing, Australia.

ParticiphCToN. 2014, Juhy 23. Available from httpx/Www. participaction.com/ get-informed/physical-activity guidelines. [Accessed 23 july 2014 ]

Paterson. D., and Warburton. D. 2000 . Physical Activity and Punctional Limitations in older Adults: A Systematic Review Related to Canada's Physical Activity Guidelines. Int. J. Behav. Nutr. Phys. Act. 7:38 doi-10.1186/2479-58687.38. FMID: 20459782 .

Faterson, D.H., jones, C.R., and Rice, C. 2007. Ageing and physical activity: evidence to develop exercise recommendations for older adults. Appl. Physiol. Nutr. Metab. 32/52E) 569-5108. doi 10.1139/H07-111. PMID 18213941.

Plotnikoff, R.C., Todosijczuk, L., Faulkner, G., Pickering, M.A., Cnagy, S., Chad, K. et al. 2009. Particip.MCToN: Baseline assessment of the "new FarticiprCnoN":
A quantitative survey of Canadian organizational awareness and capacity. Int. 1. Behas. Nutr. Phys. Act 6it: 1-12 doi-10 1186/1479-5068-6-1. FMID:19123927. khodes, R.E., and Pfaeffli, LA. 2010 . Mediators of physical activity behariour change among adult noor-clinical populations: a review update. int. J. Behav. Nutr. Phys. Act. 7it: 37. doi:10.1156/1479-5868-7.37. FMD:20459781.

Sharratt, M.T., and Hearst, WE. 2007. Canada's physical activity guides: background, process, and development. Appl. Physiol. Nutr. Metab. 3252E: 59s15. doi:10.1139/H07-124. FMID: 18213939 .

Spence, JC. Plotnikot, R.C., and Mummery, W.X. 2002. The awareness and use of Canada's Physical Activity Guide to Healthy Active Living Can. I. Public Health. 9393): 394-396. PMID:12353464.

Timmons, B.W., Naylor, P.f, and Pfeiffer, K.A. 2007. Physial activity for preschool children - bow much and how AppL. Ftysiol. Nutr. Metab. 32(S2E):

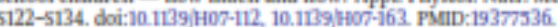

Timmons, B. W, LeBhnc, AC, Carson, $V_{\text {. }}$ Connor Corber, S. Dillman, $C$. Janssen, 1, et al. 2012 . Systematic review of physical activity and health in the early years (aged 0-4 years). Appl. Physiol. Nutr. Netab. 3774) 773-792 doi:

Tremblay, M.S., Shephard, R.J., and Brawley, L.R. 2007a. Besearch that informs Canada's physical activity guides: an introduction. Appl. Physiol. Nutr. Setab. 3252E: 51-58. doi:10.1139/HO0-104. FMID:19377534.

Tremblay, M.S., Shephard, R.J., Brawley, L.R., Cameron, C., Craig, C.L., Duggan, M., et al, 2007b. Physical activity guidelines and guides for Canadi$1139 /$ H07-125. PMD: 18213951 .

Tremblay, M.S. Colley, R.C. Saunders, T.j, Healy, G.N. and Owen, N. 20t0a. inglications of a sedentary lifestyle. Appl. Physiol. Nutr. Metab. 396): 725-740. doi:10.1139/H10-079. FMuD:21164543.

Tremblay, M.S., Kho, M.E., Tricco, A.C, and Duggan, M. 20006. Process description and evaluation of Canadian Physical Activity Cuidelines developenent. int. J. Behav. Nutr. Phys. Act. 7il: 42 doi.10.1186/1479-5868-7-42. PMID20459786.

Tremblay, M.S., LeBlanc, A.G. Janssen, 1. Kho, M.1. Hicks, A. Murumets, K., et al. $201 \mathrm{~h}$. Canadian Sedentary Behaviour Cuidelines for Children and Youth. Appl

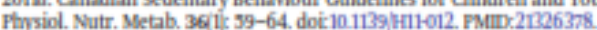

Tremblay, M.S., LeBlanc, A.C., Kho, M.E., Saunders, T.J., Larouche, R. Colley, RC., et al. 201tb. Systematic review of sedentary behaviour and health indicators in schook-aged children and youth. Int. J. Behav. Nutr. Ftys. Act. (1): 98 . doi.10.1186/1479-5368-898. PMID-21936895.

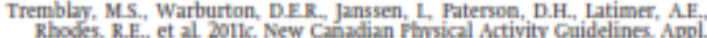
Physiol. Nutr. Metab. 36/1): 36-46. doi:10.1139/H11-009. PMDD:21326376.

Tremblay, M.S., LeBlanc, A.C., Carson, V., Choquette, L., Connor, Corber, S. Dillman, C, et al. 2012n. Canadian Physical Activity Cuidelines for the Early Years (agrd 0-4 yearsi. AppL. Physiol. Nutr. Metab. 37|21:345-356. doi:10.1139| h2012-018. PMID:2244s600

Tremblay, MS., LeBlanc, A.G., Carson, V., Choquette, L. Connor Corber, S., Dillman, C, et al. Canadian Society for Exercise Ftysiology. 2012b. Canadian Sedentary Behaviour Guidelines for the Earhy Years (aged 0-4 years). Appl. Physiol. Nutr. Setab. 372): 300-391. doi:-10.1139/h2012-019. FMID.22448609.

Warburton, D.E.R., Katzmarryk, P.T, Rhodes, R.E. and shephard, R. 2000 Evidence-informed paysical activity guidelines for Canadian adalts. Appl.

Warburton, DE, Charlesworth, S., Ivey, A, Nettlefold, L, and Bredin, 5.5. 2010. A systematic review of the evidence for Canada's Physical Activity Guidelines for Adults. Int. J Behav. Nutr. Phys. Act. 7: 39. doi: 10.1186/1479-5868-7-39. PMad:20459783.

Who. 2010. Clobal recommendations on physical activity for health. Available from http / wwow.who.int/dietphysicalactivity/publications/9789241599979/ en'index.htmL. [Accessed 28 February 2013.]

Young, T.K., and Katzmarrik. P.T. 2007. Physical activity of Aboriginal people in Canada. Appl. Ftrsiol. Nutr. Metab. 3252E| 5148-5160. dot:10 1139 H07-110. PMAD:18213945.

\section{Appendix A}

Table A1. Search strategies.

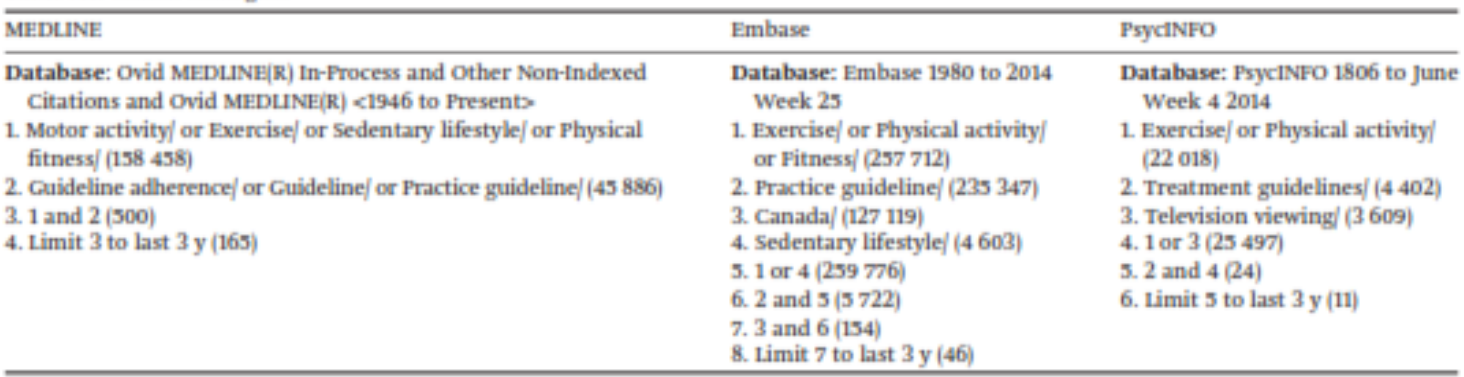

Note: No. articles identified at each stage is presented in parentheses. 


\section{Appendix B}

Table B1. Survey questions.

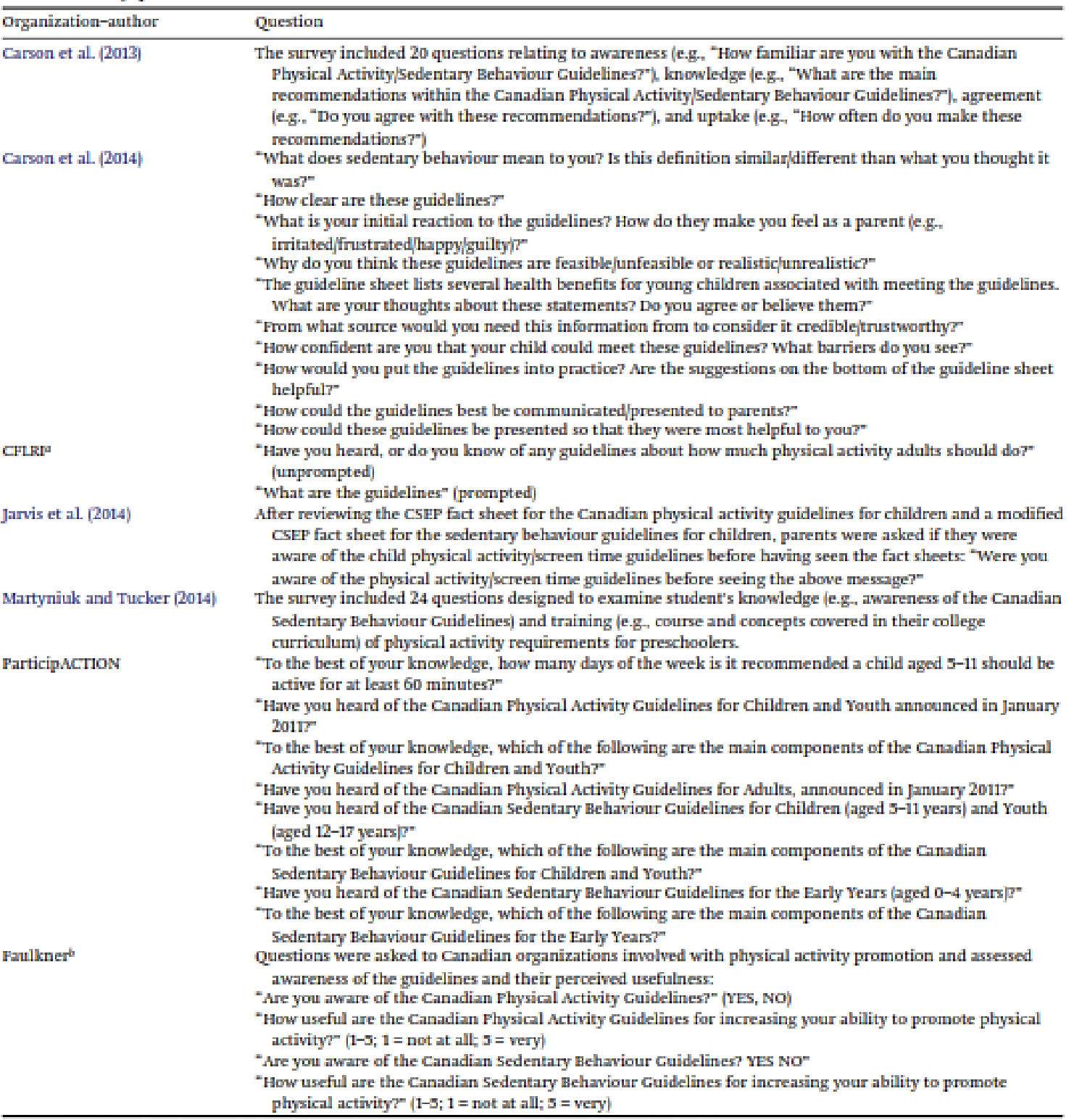

Note: CFLR, Canadian Fitness and Lifestyle Besearch Institute; CEP, Canadian Society for Exercise Physiology-

-Unpublished interim GIRI PAM data, 2014.

G. Palkner 2014, personal communication.

\section{References}

Carson, V., LeBhanc, C.M., Moerau, E., and Tremblay, M.S. 2013. Faediatricians awareness of, agreement with and use of the new Canadian Physical Activity and Sedentary Behaviour Guidelines for children and youth zero to 17 years of age. Paediatr. Child Health 1s(10) 538-542

Carson, $\mathrm{V}_{-}$Clark, M., Berry, $\mathrm{T}_{3}$ Holt, N., and Latimer-Cheung, A.E. 2014. A qualitative examination of the perceptions of parents on the Canadian Sedentary Behaviour Guidelines for the early years. Int. J. Behav. Nutr. Phys. Act. 11(1) 65. doi:10.1156/1479-5868-11-65. fanis, fw. 2014. Imestigating the messge believability as a determinsut of parent's intentions to support theirchildren in meeting physial activity and screen time guidelines. Master's Thesis, Queen's University, Kingston, Ont, Canada.

Martyniuk, O.J., and Tucker, P. 2014. An exploration of Early Childhood Educ. tion students' knowledge and preparation to facilitate phrsical activity for tion students' knowiedge and preparation to facilitate ptysical activity for preschoolers a cross-sectional study.

FarticiphCToN. 2014, July 23. Available from httpe/[wow.participaction.com/ get-informed/physical-activity-guidelines]- [Accessed 23 july 2014 |

Tublished by NRC Besearch Fress 
Appendix C

Consent forms and ethics approval 


\section{Children's Hospital of Eastern Ontario Research Institute Ethics Approval}

- Children's Hospital of Eastern Ontario

Centre hospitalier pour enfants de l'est de l'Ontario

\section{CHEO Research Ethics Board Annual Re-Apnroval Notice}

\begin{tabular}{|c|c|}
\hline Principal Investigator & Dr. JeanrFailippe Clapat \\
\hline RE B Protocol Number & Aิ1 $1 / 44 \mathrm{X}$ \\
\hline Protocol Title & International Stady of Childhoed Obesity, Lifestyle ind the Environment (ISCOLE) \\
\hline Department or PSU & Healthy Active Living and Obesity \\
\hline Approval Date & June 16,2012 \\
\hline Valid Dntil & June 15, 2013 \\
\hline $\begin{array}{l}\text { Docwments Reviewed \& } \\
\text { Approved }\end{array}$ & 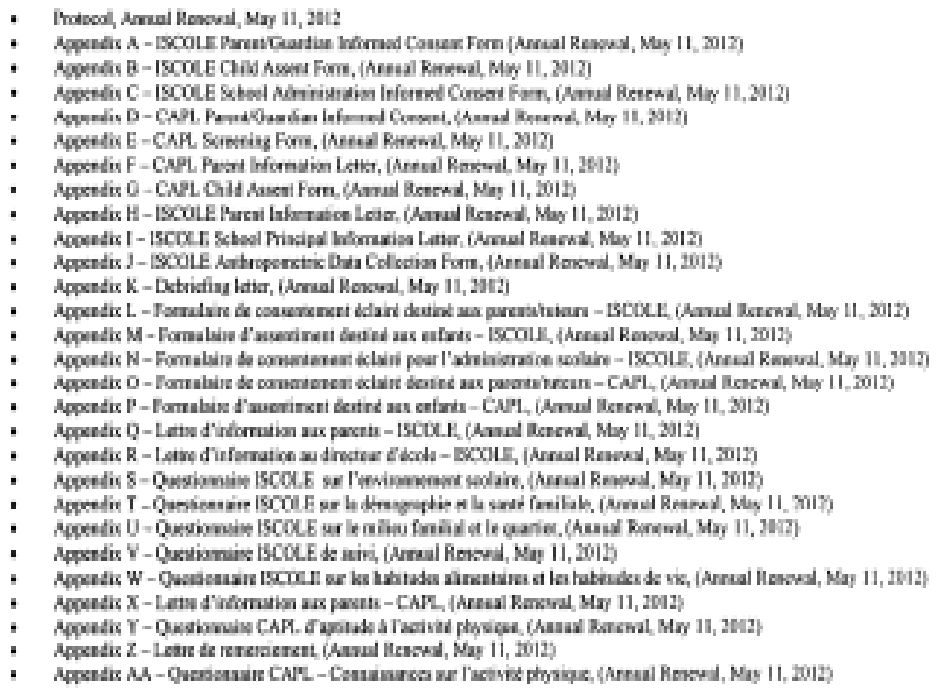 \\
\hline
\end{tabular}

This is to notify you that the CHEO REB has granted approval to the rencwal for the above named resetech study for a period of cece year. The rtnewal was reviewed and approved by the Chair. Decisions made by the Cheir under dekgetod fovicw are netiflod by the full Board at its subsequent meeting

In fulfilling its mandaic, the CHEO REB is guided by: Tri-Council Policy Statenent; ICH Geod Clinical Practice Practices: Coesolidased Guideline; Applicable laus and regulations of Ontario and Canada (c.g. Health Casada Divisioe 5 of the Food and Drug Regulutions \& the Food and Drugs Ad - Medical Devioes Regulations).

Anaual Renewal is granted with the understanding that the investigater agrees to eomply with the following requirements:

- The investigater most coeduct the stody in compliance with the pootocol ats any abditional conditions set out by the Board.

- Tre investigawor must not implentens any deviation from, or changes to, the prowocol without the appeoval of the Rea, or

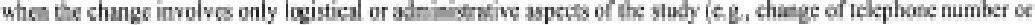
reseirch stafm.

- For all other research studies, investipasos mast prometly report to the REB all unexpecied and unioward occurrences (including the loos of theft of study data and ochar such privacy breaches).

- Investigators must submit an annual nenewal nepoet to the REB 30 days prior to the expiration dane staked ubove.

- Investigators must submit a final report at the cosclusice of the shaly,

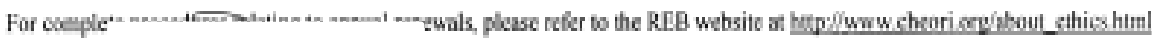
Requats,

Dr. Carole Gientile, Cr.sych.

Chair, Resegrch Ethics Board

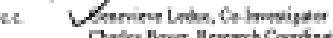

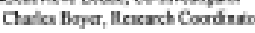

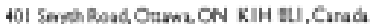

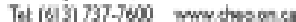

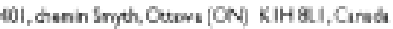

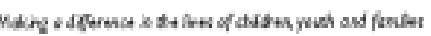

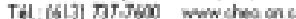

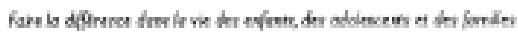




\section{University of Ottawa Ethics Approval}

File Number: H02-14-23

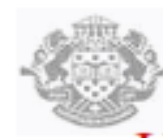

Date (mm/dd/yyy): 03/03/2014

\section{Université d'Ottawa University of Ottawa}

This is to confirm that the University of Ottawa Research Ethics Board identified above, which operates in accordance with the Tri-Council Policy Statement and other applicable laws and regulations in Ontario, has examined and approved the application for ethical approval for the above named research project as of the Ethics Approval Date indicated for the period above and subject to the conditions listed the section above entitled "Special Conditions / Comments".

During the course of the study the protocol may not be modified without prior written approval from the REB except when necessary to remove participants from immediate endangerment or when the modification(s) pertain to only administrative or logistical components of the study (e.g. change of telephone number). Investigators must also promptly alert the REB of any changes which increase the risk to participant(s), any changes which considerably affect the conduct of the project, all unanticipated and harmful events that occur, and new information that may negatively affect the conduct of the project and safety of the participant(s).

Modifications to the project, information/consent documentation, and/or recruitment documentation, should be submitted to this office for approval using the "Modification to rescarch project" form available at: httn://www ressearch.uottawa calethics/forms.html.

Please submit an annual status report to the Protocol Officer four weeks before the above-referenced expiry date to either close the file or request a renewal of ethics approval. This document can be found at: http://www.research.uottawa.ca/ethics/forms.html.

If you have any questions, please do not hesitate to contact the Ethics Office at extension 5387 or by e-mail at: ethics@uOttawa.ca.

\section{Signature:}

Kim Thompson

Protocol Officer for Ethics in Research

For Daniel Lagarec, Chair of the Sciences and Health Sciences REB

2

550 , rue Cumberland, pièce $154 \quad 550$ Cumberland Street, room 154 Ottawa (Ontario) K1N 6N5 Canada Ottawa, Ontario K1N 6 N5 Canada (613) 562-5387 • Téléc./Fax (613) 562-5338. 


\section{$\underset{\text { Burean d'éthique et d'intigrite de la recherche }}{\text { Universitá }}$ University of Ottawa}

\section{Ethics Approval Notice}

\section{Health Sciences and Science REB}

\section{Principal Investigator / Supervisor / Co-investigator(s) / Student(s)}

$\begin{array}{llll}\text { First Name } & \text { Last Name } & \text { Affiliation } & \text { Role } \\ \text { Mark } & \text { Tremblay } & \text { Health Sciences / Other } & \text { Supervisor } \\ \text { Allana } & \text { LeBlanc } & \text { Health Sciences / Population Health } & \text { Student Researcher }\end{array}$

File Number: H02-14-23

Type of Project: PhD Thesis

Title: Correlates of Overall Sedentary Time, and Specific Sedentary Behaviours in Children

Approval Date (mm/dd/yyyy)

Expiry Date (mm/dd/yyyy)

$03 / 03 / 2014$

$03 / 02 / 2015$

(la: Approval, Ib: Approval for initial stage only)

Special Conditions / Comments:

$\mathrm{N} / \mathrm{A}$

\section{Approval Type}

la 


\section{Université d'Ottawa University of Ottawa \\ Bureau d'éthique et d'intégrité de la recherche \\ Office of Research Ethics and Integrity}

\section{Ethics Approval Notice}

\section{Health Sciences and Science REB}

Principal Investigator / Supervisor / Co-investigator(s) / Student(s)

$\begin{array}{llll}\text { First Name } & \text { Last Name } & \text { Affiliation } & \text { Role } \\ \text { Mark } & \text { Tremblay } & \text { Health Sciences / Others } & \text { Supervisor } \\ \text { Allana } & \text { LeBlanc } & \text { Health Sciences / Others } & \text { Student Researcher }\end{array}$

File Number: H02-14-23

Type of Project: $\mathrm{PhD}$ Thesis

Title: Correlates of Overall Sedentary Time, and Specific Sedentary Behaviours in Children

Renewal Date (mm/dd/yyyy)

03/03/2015

(Ia: Approval, Ib: Approval for initial stage only)

Special Conditions / Comments:

N/A
Expiry Date (mm/dd/yyyy)

$03 / 02 / 2016$
Approval Type

Ia 


\section{Université d'Ottawa University of Ottawa \\ Bureau d'éthique et d'intégrité de la recherche \\ Office of Research Ethics and Integrity}

This is to confirm that the University of Ottawa Research Ethics Board identified above, which operates in accordance with the Tri-Council Policy Statement (2010) and other applicable laws and regulations in Ontario, has examined and approved the ethics application for the above named research project. Ethics approval is valid for the period indicated above and subject to the conditions listed in the section entitled "Special Conditions / Comments".

During the course of the project, the protocol may not be modified without prior written approval from the REB except when necessary to remove participants from immediate endangerment or when the modification(s) pertain to only administrative or logistical components of the project (e.g., change of telephone number). Investigators must also promptly alert the REB of any changes which increase the risk to participant(s), any changes which considerably affect the conduct of the project, all unanticipated and harmful events that occur, and new information that may negatively affect the conduct of the project and safety of the participant(s). Modifications to the project, including consent and recruitment documentation, should be submitted to the Ethics Office for approval using the "Modification to research project" form available at: http://research.uottawa.ca/ethics/submissions-and-reviews.

Please submit an annual report to the Ethics Office four weeks before the above-referenced expiry date to request a renewal of this ethics approval. To close the file, a final report must be submitted. These documents can be found at: $\underline{h t t p: / / r e s e a r c h . u o t t a w a . c a / e t h i c s / s u b m i s s i o n s-a n d-r e v i e w s . ~}$

If you have any questions, please do not hesitate to contact the Ethics Office at extension 5387 or by e-mail at: ethics@uOttawa.ca.

\section{Signature:}

Jasmine Sarazin

Ethics Coordinator

For Catherine Paquet, Director of the Office of Research Ethics and Integrity 


\section{Sample Child Assent Forms}
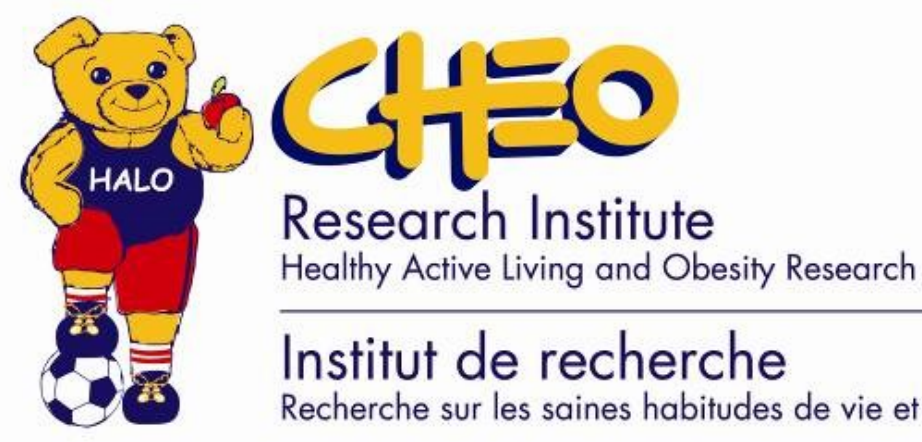

Institut de recherche

Recherche sur les saines habitudes de vie et l'obésité

ISCOLE Child Assent Form - School

\section{What are they asking me?}

I am being invited to take part in a research study. The researchers want to know about my living habits and the world around me. They will be comparing me and my classmates with children from all around the world.

\section{Who is doing this research?}

Dr. Mark Tremblay, Dr. Jean-Philippe Chaput, Dr. Geneviève Leduc and Mr. Charles Boyer. They work at the Children's Hospital of Eastern Ontario in research. You can contact them by telephone at 613-737-7600 extension 4192. Your school board, school principal and school teacher have all approved this study.

\section{What will they do to me?}

They want to take my height, my weight, and a couple measurements around my belly and arms. I will have to remove my shoes and socks for the height and weight and I will also have to lift my shirt up a little bit so that they will be able to measure my belly. I will keep my underwear, pants and shirt on at all times. Also, they want to ask me questions about watching TV, playing computer games, play time, going to school, activities, sleep, what I eat and how I feel. They are also going to ask me to wear one or two little device(s) called accelerometer(s) (like a pedometer) that I wear around my waist. The little device(s) can measure how many steps I take in a day. I can only take them off if I go in water because they are NOT waterproof. 

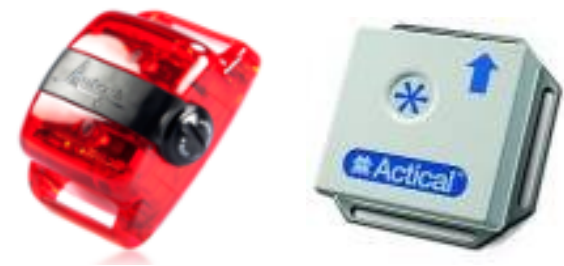

Pictures of accelerometers

\section{Where is this happening?}

The study will take part at my school and my teacher and principal know about the research. If I don't want to participate I will be able to remain in my classroom with my teacher.

\section{Why?}

The researchers are doing this research because children around the world are not getting enough physical activity. There are differences in children around the world and this research will find out how those differences influence physical activity.

\section{Will it hurt?}

No, the study will not hurt you. If the little device(s) is/are broken there may be small pieces that break apart that could cause you and children younger than you to choke on them. For this reason you should keep the little device(s) away from children who are 3 years old or younger.

\section{What do I get if I participate?}

By joining the study I will get no extra benefits. The researchers will get information that could help children all across the world. By wearing the second monitor I will get my results compared with other Canadian children.

\section{Do I have to participate?}

I don't have to participate if I don't want to. My participation in this study does not affect any of my marks, and I can decide to stop the study at any time.

\section{Will anybody know my results?}


The results they get won't be shared with anyone except me and my parents. They will give me a private number so no one will know my name when they compare the results.

\section{Child Assent for Study Participation - School}

(your name)

$\square$ I do not want to wear the second monitor.

I have read and understood the attached information sheet / had the attached information sheet verbally explained to me. I have been told about the study and have had the chance to ask questions. I know I am free to withdraw from the study at any time, or not answer questions that make me feel uncomfortable. My physical education grade and my treatment at $\mathrm{CHEO}$ will not be affected if I do or do not participate. I have received a copy of the information sheet and assent form.

Name of Participant

Signature of Participant

Date

More information can be found at: http://www.cheori.org/halo/ 


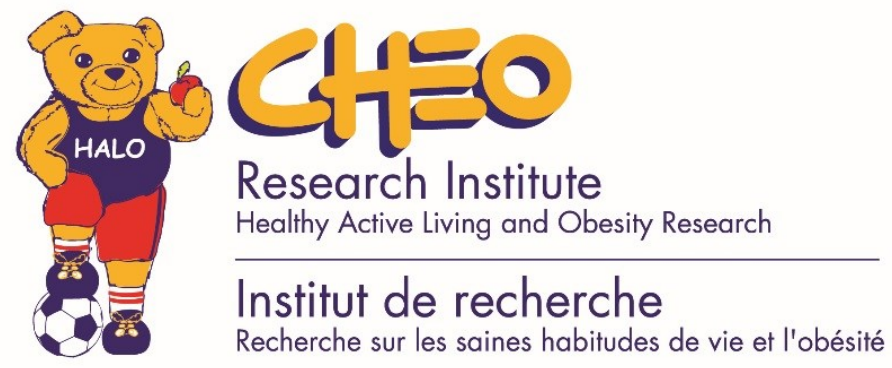

\section{CAPL Child Assent Form - School}

\section{What is this research called?}

Fitness and movement skill testing

\section{Who is doing this research?}

Dr. Pat Longmuir, Dr. Mark Tremblay, Dr. Jean-Philippe Chaput, Dr. Geneviève Leduc and Mr. Charles Boyer. They work at the Children's Hospital of Eastern Ontario in research. You can contact them by telephone at 613-737-7600 extension 4192. Your school board, school principal and school teacher have all approved this study.

\section{Why are we doing this study?}

We are doing this study because fitness and movement skills are very important to be healthy and active. By performing this second part of the study, we will be able to know a lot more about you and how all your lifestyle and environment effect your physical activity.

\section{What will you do during the study?}

You will do many activities that are like being in gym class. We will ask you to answer some questions. In the gym we will ask you to try each of these tasks:

- Run through an obstacle course with jumping, throwing and kicking a ball.

- Squeeze a handle as hard as you can with each hand.

- Keep a straight body while leaning only on your toes and elbows.

- Reach toward your toes while sitting with your legs straight.

- Run laps back and forth across the gym, starting slowly and then getting faster.

- Answer questions about physical activity. 
All of the research activities will take place at your school and your teacher will be there too. The activities will be done in your classroom and in the gymnasium. If you do not want to be in the research study that's okay, you will just stay with your teacher.

\section{Are there good or bad things about being in this study?}

We do not expect bad things to happen to children who participate in this study. Since you will be running and doing other activities, you might fall or bump into something. It will be the same as gym class. We will not give you anything for being in this study but what we learn might help other children to be healthier one day.

\section{Who will know that I am in this research study?}

All of your written information will be stored safely, and your personal information will stay private. You don't have to participate if you don't want to, and the information we get won't be shared with anyone except you and your parents. Being in this study will not change your marks, and you can decide to stop the study at any time.

\section{CAPL Child Assent}

I, (Your Name), agree to participate in this research study.

I have read and understood the study information or the information has been explained to me. I have been told all about the study and have had a chance to talk about it. I understand that I can change my mind and stop the study at any time. I also know that I do not have to answer questions or do things that make me uncomfortable. I know that my school marks will not be affected because I am doing this research. I have received a copy of the study information and consent form. 
More information can be found at: http://www.cheori.org/halo/ 


\section{Sample Parent Consent Forms}

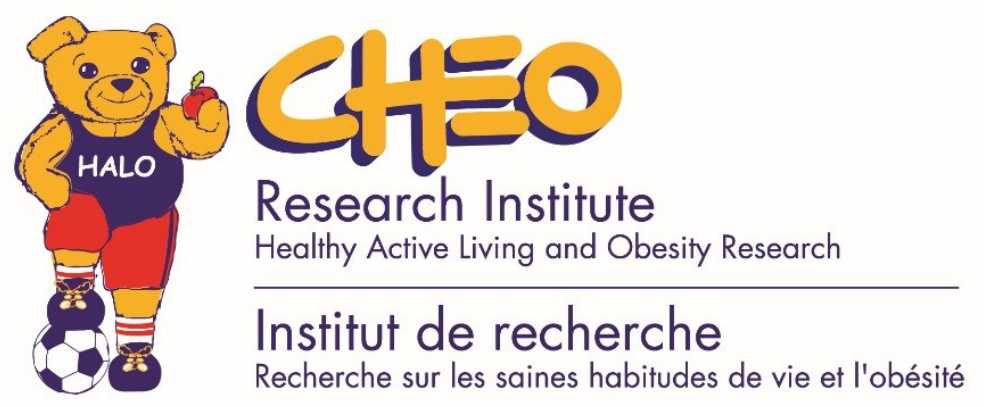

\section{Parent/Guardian Informed Consent Form -School}

Study Title: International Study of Childhood Obesity, Lifestyle and the Environment (ISCOLE)

\section{Investigators:}

\begin{tabular}{|c|c|}
\hline Principal Investigator: & $\begin{array}{l}\text { Dr. Jean-Philippe Chaput, PhD } \\
\text { CHEO Research Institute } \\
\text { Healthy Active Living and Obesity Research Group } \\
\text { (613) 737-7600 \#3683 } \\
\text { jpchaput@cheo.on.ca }\end{array}$ \\
\hline Co-Investigator: & $\begin{array}{l}\text { Dr. Mark Tremblay, PhD } \\
\text { CHEO Research Institute } \\
\text { Healthy Active Living and Obesity Research Group } \\
\text { (613) 737-7600 \#4114 } \\
\text { mtremblay@cheo.on.ca }\end{array}$ \\
\hline Project Manager: & $\begin{array}{l}\text { Dr. Geneviève Leduc, PhD } \\
\text { CHEO Research Institute } \\
\text { Healthy Active Living and Obesity Research Group } \\
\text { (613) 737-7600 \#4192 } \\
\text { gleduc@cheo.on.ca }\end{array}$ \\
\hline
\end{tabular}

\section{Background and Rationale}

You are being invited to join this study because your child is registered in Grade 5 with a school participating in this study. Your child will be among 500 other children from the Ottawa area that will participate in this study. This exact study is being conducted in 11 other countries around the world. The main reason for the study is the fact that children are increasing in unhealthy weight around the world at a high rate, and changes in lifestyles have likely been the main cause. Childhood weight gain is the result of many factors and these factors play a large role in how youth maintain their weight into their teen years and onto adulthood. Some of these factors are local 
and national policies; the neighborhood; home and school environment; diet and lifestyle behaviors; and personal characteristics of the child. There is a need for an international study to assess these lifestyle behaviors and how they influence weight gain and obesity in children. Your school board and school are supportive of this study and your principal and child's teacher are fully aware that it is taking place.

\section{Why is this work important?}

Children around the world are getting heavier to the point where a child's weight is affecting their current and future health. To understand why children are gaining excess weight, this international study can answer about how people live affects how much they weigh. This study will measure important factors that make up the child as in the physical makeup of the child (i.e. height, weight), personal lifestyle (i.e. what they like to do) and demographic characteristics (i.e. family income, ethnicity) and the childhood environments (i.e. access to parks). In being an international study, all of these factors will be compared across countries from the major world regions (Eurasia \& Africa, Europe, Latin America, North America, and the Pacific). The results obtained will help create solutions to stop childhood weight gain that can be adapted specifically to each country.

\section{Study Procedures:}

The research team will ask you (the parent or legal guardian) to complete the ISCOLE Neighborhood and Home Environment Questionnaire, which will document your perception of your child's home, neighborhood, and school environments. In addition, you will be asked to complete the ISCOLE Demographic and Family Health Questionnaire, which will ask personal information (such as education, family income, height and weight) about yourself and other personal information about your family and family history that is indirectly related to the health of your child. With your permission, we would also like to be able to measure your child's health over time. That is why we are also asking you to fill a Tracing Questionnaire, which would allow us to communicate with you in the future if further testing were to take place. However, we do not currently know if we will have the resources needed to be able to do these future tests.

Your child will bring home the questionnaires. You can complete the questionnaires at home. It will take about 15 minutes to complete the questionnaires. You will be asked to return the questionnaires in the pre-stamped, self-addressed envelope to CHEO.

All children enrolled in the study will have the same measurements taken. All measurements will be confidential and no one, other than the researchers involved in the study, will see the results. School personnel will also be restricted from viewing your child's measurements.

Taking your child's measurements will be coordinated with school administrators to not conflict with important school activities or tests. Trained research staff will take your child's measurements in a private area at the school determined by the school principal. The measures will be taken individually so that information remains private from other students. Your child should wear his/her usual clothes for the day of the measurements. We will ask your child to remove his/her shoes and socks for height and weight. We will also ask that he/she lifts up his/her shirt over the waist for the waist circumference measurement. Children will keep underwear, pants and shirt on at all times.

The measurements will include: 
1. Weight

2. Height (standing and sitting)

3. Body fat - this number is calculated while your child is standing on a weight scale

4. Waist and arm circumferences

5. Questionnaire about dietary habits and exercise

In addition, your child will wear at least one, with the possibility of wearing two, physical activity monitor(s) for 8 days, 24 hours a day, to measure his/her normal physical activity. Your child should only remove the monitor(s) when in water, as the monitor(s) is/are NOT waterproof. The monitor(s) is/are on a flexible belt that is around the waist. A research staff member will call you twice during the monitoring time to answer questions you might have. By agreeing to wear a second monitor, we will be able to provide your child with their results compared to national norms from the Canadian Health Measures Survey (CHMS) in addition to other ISCOLE findings and measures.

\section{Risk and Benefits:}

There are no known risks to study participation.

The special scale, which we use for measuring weight and percent body fat, sends a weak electrical current through the body during measurement. Children who have internally implanted medical devices, such as pacemakers or other types of internal medical devices should not use this equipment due to the risk of malfunction to the device that may be caused by the weak electrical current. If your child is wearing such a device, you will be asked to check a box in the Consent section of this document and he or she will be weighed using a traditional scale and will not have his or her percent body fat measured.

If the physical activity monitor is disassembled or broken, there could be small pieces dislodged which causes a choking hazard to children under the age of three. For this reason, children under the age of 3 years old should be kept out of reach of the device. You will receive no payment for participating in this study. Although you and your child will not receive direct benefits from joining this study, the information gained from this study may help other children struggling with issues related to physical activity participation. You and your child's participation in this study are voluntary and you are both free to withdraw at anytime from this study. Neither participation nor withdrawal from the study will affect your child's physical and health education grade or the care you receive at CHEO.

\section{Confidentiality:}

The data collected in this study will be locked in a safe place. All information that you and your child provide will be numbered and will not contain your name. A list of names and matching codes will be stored separately. Only the staff involved in this research study will have access to the records, with the exception of the CHEO Research Ethics Board who has access to records for auditing purposes. All study data will be manually entered into a secure web-based database maintained under the auspices of the Coordinating Center in Baton Rouge, in the United States.After 7 years of storage, all records will be destroyed. Overall results may be published for scientific purposes, but participant identity will remain confidential. Your information is confidential. If we learn that a child was being harmed or at risk of being harmed we will notify you as well as the proper authorities. 


\section{Questions about the study:}

If you have any questions please contact Dr. Geneviève Leduc at 613-737-7600 x4192. The Ottawa-Carleton District School Board (your child's school board) and your child's school principal have approved this research. This study has also been reviewed and approved by the CHEO Research Ethics Board (REB). The CHEO REB is a committee of the hospital that includes individuals from different professional backgrounds. The Board reviews all research that takes place at the hospital. Its goal is to ensure the protection of the rights and welfare of people participating in research. The Board's work is not intended to replace a parent or child's judgment about what decisions and choices are best for them. You may contact the Chair of the REB, for information regarding patient's rights in research studies at (613) 737-7600 \#3272, although this person cannot provide any health-related information about the study. 


\section{Parental Guardian Consent}

(Your Name)

the parent/guardian of

(Your Child's Name)

$\square$ Consent for myself and give consent for my child's participation in the above study.

$\square$ My child is wearing a pacemaker or another internal medical device. He or she should be weighed using a traditional scale and should not have his or her percent body fat measured.

Do not consent for my child wearing a second physical activity monitor.

$\square$ Do not consent for myself or do not give consent for my child's participation in the above study.

I have read and understood the attached information sheet / had the attached information sheet verbally explained to me. I have been fully informed of the details of the study and have had the opportunity to discuss my concerns. I understand that I am free to withdraw my child at any time or not answer questions that make us uncomfortable, and that my child's educational status or treatment from CHEO will not be affected if I do. I have received a copy of the information sheet and consent form. The study volunteer is a child and I certify that I am his/her legal guardian.

Name of child

Name of Parent/

Signature of Parent/

Date

Guardian

Guardian

More information can be found at: http://www.cheori.org/halo/ 

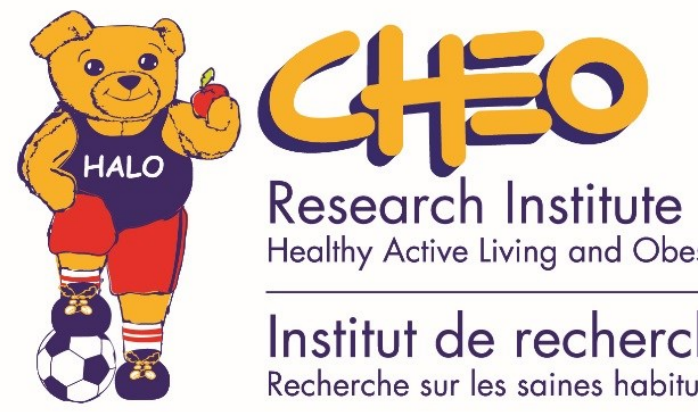

Healthy Active Living and Obesity Research

Institut de recherche

Recherche sur les saines habitudes de vie et l'obésité

CAPL Parent/Guardian Informed Consent Form - School

\begin{abstract}
What is the title of this research study?
Canadian Assessment of Physical Literacy
\end{abstract}

Who is doing this research?

Dr. Jean-Philippe Chaput, Principal Investigator

Healthy Active Living and Obesity Research Group, CHEO - Research Institute

Tel: (613) 737-7600 \#3683, email: jpchaput@cheo.on.ca

Dr. Mark Tremblay, Co-Investigator

Healthy Active Living and Obesity Research Group, CHEO - Research Institute

Tel: 613-737-7600 \#4114, email: mtremblay@cheo.on.ca

Dr. Pat Longmuir, Co-Investigator

Healthy Active Living and Obesity Research Group, CHEO - Research Institute

Tel: 613-737-7600 \#3908, email: plongmuir@cheo.on.ca

Dr. Geneviève Leduc, Project Manager

Healthy Active Living and Obesity Research Group, CHEO - Research Institute

Tel: 613-737-7600 \#4192, email: gleduc@cheo.on.ca

\title{
Why are we doing this study?
}

It is important for children to develop physical abilities so that children are able to move efficiently, effectively and safely. Physical education is where children learn these abilities and develop knowledge and understanding on how to lead a healthy, active lifestyle. It is important to directly measure these abillities to discover exactly how they impact a child's health and also how a childs lifestyle and environment can influence physical abilities. This study is also being done in 3 other countries and comparing the results between coutnries will allow us to understand exactly how environement impacts a child's physical abilities.

\section{What will your child do during the study?}

The Canadian Assessment of Physical Literacy (CAPL) includes many activities that are similar to what your child would typically do during the school day. Before your child tries any of the study activities, we will ask your child whether they want to participate. Your child can say either "Yes" 
or "No", and their choice will be respected even if you want your child to participate. If your child agrees to participate, your child will then be asked to complete each of the following tasks:

- Obstacle Course - includes jumping, running, hopping, catching, throwing and kicking balls while running

- Grip Strength - squeezing a hand-grip dynamometer (gage)

- Plank - a core strength exercise commonly used in yoga-like activities and sport training: holding a Push Up position while resting on toes and forearms

- Sit and Reach - Reach toward the toes while sitting with their legs straight, to measure flexibility.

- PACER (Beep Test) - Run laps back and forth across the gym, starting at a slow speed and gradually getting faster. They will continue running until they are too tired or do not wish to continue running at the faster speed.

- Questionnaire - Answer questions about physical activity by writing their answers on a questionnaire or using a computer to answer the questions. The questions will tell us what children know about physical activity, physical fitness and the skills they need to be active. The questions will also ask about your child's interest in physical activity.

Children who participate in this research will perform each of the study activities when the researchers come to their school. All of the research activities will take place at your child's school and your child's teacher will be present at all times. Most activities will take place in the gymnasium, during physical and health education class.

If you choose not to allow your child to participate in this study, your child will be supervised by their own teacher and engaged in appropriate school-focused activities while the other children in the class are completing the study.

\section{Who can participate in this research?}

Only children who have participated in the ISCOLE research project will be asked to participate. We are asking 500 children in grade 5 (approximately 10 years old), to participate in this research.

Your child's teacher and the principal of your child's school are interested in having your child's class participate in this research. The Ottawa-Carleton District School Board (your child's school board) and your child's school principal have approved this research.

\section{Could something bad happen to my child during this study?}

We do not expect bad things to happen to children who participate in this study. All the activities for the study are similar to what your child does in physical education class. As with any type of physical activity, there is a small risk of falling or getting hurt. However, all the research equipment is similar to what your child uses in physical education and safety is our first priority. All study personnel are trained in First Aid and CPR, and in the event of an injury or emergency, standard school policies will be followed.

In the unlikely event that your child is injured as a direct result of participating in this research, the normal legal rules about compensation for the injury will apply. By signing this consent form 
you are in no way waiving your legal rights or releasing the investigator and the sponsor from their legal and professional responsibilities.

\section{Will my child or family get something for being in this study?}

You and your child will not be paid or given a reward for being in this study. We cannot promise that you will get any benefit from your child's study participation. The information that we gather during this study will help us understand how physical abilities impact the overall health of children in Canada.

Your child's participation in this study is completely voluntary. You or your child are free to withdraw from this study at anytime, even after the research testing has been completed. Neither participation nor withdrawal from the study will affect your child's physical and health education grade.

\section{Who will know that my child is in this research study?}

The information we collect about your child will not identify your child. We will use a coded identification number instead of your child's name so that only the researchers will know who the information is about. The data collected in this study will be locked in a safe place. All information from your child will be numbered and will not contain your child's name. A list of names and matching code numbers will be stored separately.

It is intended that only the staff involved in this research study will have access to the research information collected during this study. However, there are specific situations where other people may be given access to the research information. A member of the Research Ethics Board at the Children's Hospital of Eastern Ontario (CHEO) may be given access to the research records for auditing purposes. There are also limits to the confidentiality of research information in situations of suspected child abuse, concerns of harm to self or others, or any request for information by court order.

The coded information collected during this research study will be stored for 7 years after all of the results of this research have been published unless you have consented to participation in longer-term follow-up studies. After that time, all records will be destroyed in the way required by Canadian research data regulations. Overall study results may be published for scientific purposes, but the identity of the research participants will remain confidential. No information that could identify your child or your child's school will be published. If we want to publish information that could identify your child or your child's school, we will contact you and ask you to sign a separate consent form for the publication.

\section{Who should I contact if I have questions about the research study?}

If you have questions about this study please contact Dr. Geneviève Leduc. Dr. Leduc can be contacted by telephone at (613) 737-7600 ext. 4192 or by email (gleduc@cheo.on.ca). This study has been reviewed and approved by the CHEO Research Ethics Board. The CHEO Research Ethics Board is a committee of the hospital that includes individuals from different professional backgrounds. The Board reviews all research done by scientists at the hospital that involves people. Its goal is to ensure the protection of the rights and welfare of people participating in research. The Board's work is not intended to replace a parent or child's judgment about what decisions and choices are best for them. You may contact the Chair of the Research Ethics Board, for information regarding participant's rights in research studies at (613) 737-7600 ext. 3272, 
although this person cannot provide any health-related information about the study. The Board could review your study records in fulfilling its roles and responsibilities.

\section{CAPL Parent/Guardian Informed Consent - School}

I,

(Your Name),

the parent/guardian of

give consent for my child to participate in this study.

(Your Child's Name)

I have read and understood the attached study information or had the attached information verbally explained to me. I have been fully informed of the details of the study and have had the opportunity to discuss my concerns. I understand that I am free to withdraw my child at any time or not answer questions that make us uncomfortable, and that my child's educational status will not be affected if I do. I have received a copy of the study information and consent form.

Name of Parent/Guardian

Signature of Parent

Date

My check mark in this box indicates that I wish to receive a copy of my child's research study results.

More information can be found at: http://www.cheori.org/halo/ 


\section{Appendix D \\ ISCOLE Diet and Lifestyle Questionnaire}

Please read every question carefully. What answer comes to your mind first?

Choose the box that fits your answer best and fill it in.

Remember: This is not a test so there are no wrong answers. It is important that you answer all the questions and that we can see your marks clearly.

You do not have to show your answers to anybody. Also, nobody who knows you will look at your questionnaire once you have finished it.

For the questions on this page, please tell about what you did last week.

1. On a school day, how many hours did you watch TV?

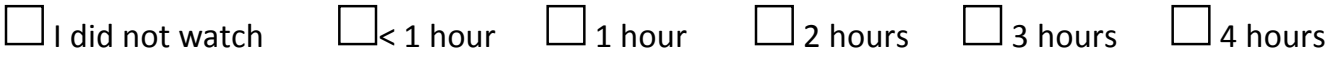
5 or more hours

TV on school

days

2. On a school day, how many hours did you play video or computer games or use a computer for something that was not school work?

$\square$ I did not play $\quad \square<1$ hour $\quad \square$ 1 hour $\quad \square$ 2 hours $\quad \square$ 3 hours $\quad \square$ 4 hours

5 or more hours

video/computer

games or use a

computer other

than for school

work on school days

3. On a school day how much time did you spend outside before school?
$\square<1$ hour
$\square 1$ hour
$\square 2$ hours $\square 3$ hours
$\square$ hours
5 or more hours 
4. On a school day how much time did you spend outside after school before bedtime?

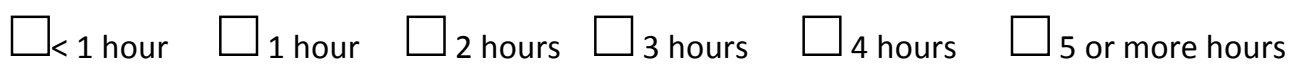

5. On a weekend day, how many hours did you watch TV?

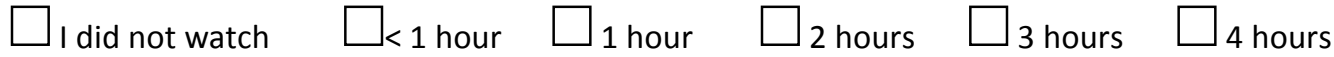

5 or more hours

TV on weekend

days

6. On a weekend day, how many hours did you play video or computer games or use a computer for something that was not school work?

$\square$ I did not play $\quad \square<1$ hour $\quad \square$ 1 hour $\quad \square$ 2 hours $\quad \square$ 3 hours $\quad \square$ 4 hours

5 or more hours

video/computer

games or use a

computer other

than for school

work on the weekend

7. On a weekend day, how much time did you spend outside?

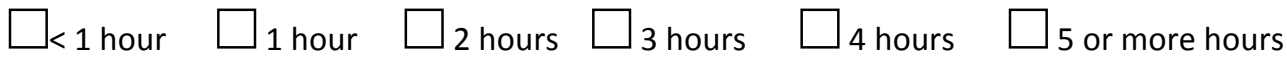

8. In the last week you were in school, on how many days did you go to physical education (PE) classes?

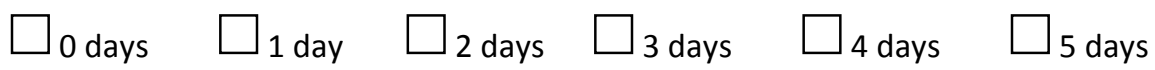

9. In the last week you were in school, the MAIN part of your journey to school was by:

walking

bicycle, roller-blade, skateboard or scooter

bus, train, tram, underground or boat

car, motorcycle or moped

other 
10. In the last week you were in school, HOW LONG did it take you to travel to school?

$\square_{<5}$ minutes $\quad \square_{5}-15$ minutes $\quad \square$ 16 - 30 minutes $\quad \square 31$ minutes to 1 hour $\square>1$ hour

11. During the past year (12 months), did you do any of these activities? (Check all that apply)

sports teams

$\square$ dance / martial arts class

$\square$ art / music class

none of these

12. During the past week (7 days), on how many days were you physically active for a total of at least $\underline{60}$ minutes per day? (all the time you spent in activities that increased your heart rate and made you breathe hard)

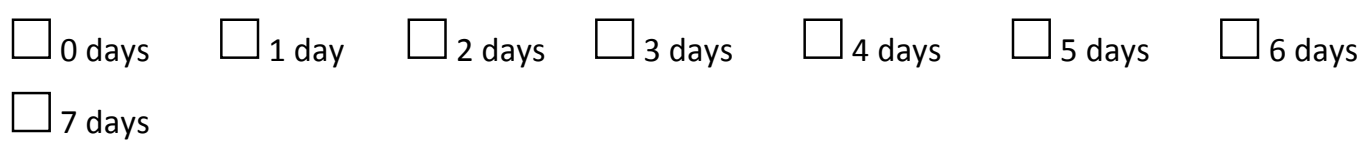

Please tick the box that most sounds like you:

Lot

4

13. I can be physically active during my free time on most days.

\section{Disagree a}

Agree a Lot

1

5

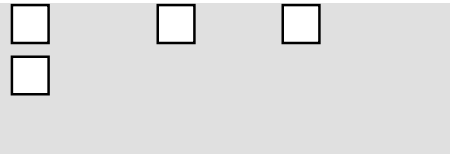

14. I can ask my parent or other adult to do physically active things with me.

15. I can be physically active during my free time on most days even if I could watch TV or play video games instead.

16. I can be physically active during my free time on most days even if it is very hot or cold outside.

17. I can ask my best friend to be physically active with me during my free time on most days.

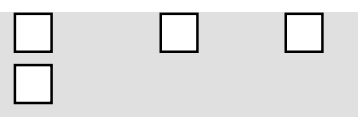


18. I can be physically active during my free time on most days even if I have to stay at home.

19. I have the coordination I need to be physically active during my free time on most days.

20. I can be physically active during my free time on most days no matter how busy my day is.

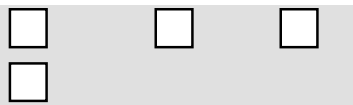

There are lots of reasons why people take part in physical activity. Please tick the box to show how much each of the reasons below is true for you:

me

$\begin{array}{llcc}\begin{array}{c}\text { Never true } \\ \text { Very true }\end{array} & \text { A little bit } & \text { Sometimes } & \text { True } \\ \text { for me } & \text { true for me } & \text { true for me } & \text { for } \\ \text { for me } & & & \end{array}$

21. I take part in exercise because other
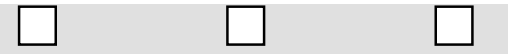
people say I should

22. It's important to me to exercise regularly

23. I can't see why I should bother exercising $\square$
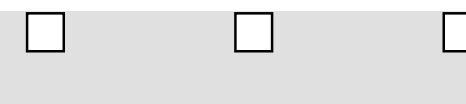

24. I feel like a failure when I haven't exercised in a while

25. I find exercise a pleasurable activity $\square$

26. During the past week, what time have you usually turned out the light and gone to sleep on school days?

$\square \square: \square \square$ AM / PM (circle AM or PM) 
27. During the past week, at what time have you usually woken up in the morning on school days?

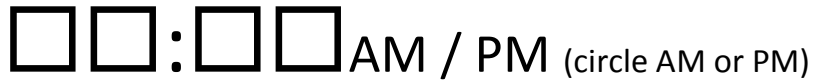

28. During the past week, what time have you usually turned out the light and gone to sleep on weekend days?

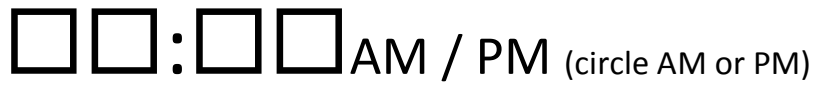

29. During the past week, at what time have you usually woken up in the morning on weekend days?

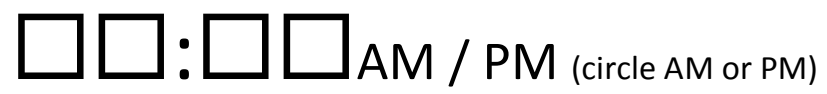

30. During the past week, how would you rate your sleep quality overall (how well you sleep)?

$\square$ very good $\square$ fairly good $\quad \square$ fairly bad $\quad \square$ very bad

31. During the past week, how would you rate your sleep quantity overall (how much you sleep)?

$\square$ very good $\square$ fairly good $\quad \square$ fairly bad $\quad \square$ very bad

32. Do you have a television in your bedroom?

$\square$ Yes $\quad \square$ No

How many times do you usually eat ... ? (Please mark only one box for each line)

\begin{tabular}{|l|c|c|c|c|c|c|c|}
\hline & Never & $\begin{array}{c}\text { Less } \\
\text { than } \\
\text { once a } \\
\text { week }\end{array}$ & Once a & $\begin{array}{c}2-4 \\
\text { week } \\
\text { days a } \\
\text { week }\end{array}$ & $\begin{array}{c}5-6 \\
\text { days a } \\
\text { week }\end{array}$ & $\begin{array}{c}\text { Once a } \\
\text { day, } \\
\text { every } \\
\text { day }\end{array}$ & $\begin{array}{c}\text { Every } \\
\text { day, } \\
\text { more } \\
\text { than } \\
\text { once }\end{array}$ \\
\hline Fruits & $\square$ & $\square$ & $\square$ & $\square$ & $\square$ & $\square$ & $\square$ \\
\hline Vegetables & $\square$ & $\square$ & $\square$ & $\square$ & $\square$ & $\square$ \\
\hline
\end{tabular}




\begin{tabular}{|c|c|c|c|c|c|c|c|}
\hline Sweets (candy/chocolate) & $\square$ & $\square$ & $\square$ & $\square$ & $\square$ & $\square$ & $\square$ \\
\hline $\begin{array}{l}\text { Regular cola or soft drinks } \\
\text { that contain sugar }\end{array}$ & $\square$ & & & $\square$ & $\square$ & $\square$ & $\square$ \\
\hline Cake, pastries, or donuts & $\square$ & $\square$ & $\square$ & $\square$ & $\square$ & $\square$ & $\square$ \\
\hline $\begin{array}{l}\text { Diet cola or diet soft } \\
\text { drinks }\end{array}$ & $\square$ & & & $\square$ & $\square$ & $\square$ & $\square$ \\
\hline Potato chips & $\square$ & $\square$ & $\square$ & $\square$ & $\square$ & $\square$ & $\square$ \\
\hline French fries & $\square$ & $\square$ & $\square$ & $\square$ & $\square$ & $\square$ & $\square$ \\
\hline $\begin{array}{l}\text { Dark green vegetables } \\
\text { (broccoli, spinach, etc.) }\end{array}$ & $\square$ & & & $\square$ & & $\square$ & \\
\hline $\begin{array}{l}\text { Orange vegetables } \\
\text { (carrots, squash, sweet } \\
\text { potato, etc.) }\end{array}$ & L & & & 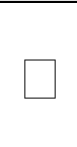 & r & & \\
\hline Fruit juice & $\square$ & & $\square$ & $\square$ & $\square$ & $\square$ & $\square$ \\
\hline $\begin{array}{l}\text { Low fat milk (1\%,2\%, } \\
\text { skim) }\end{array}$ & 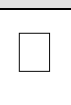 & & & 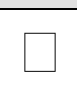 & & $\square$ & \\
\hline $\begin{array}{l}\text { Whole milk } \\
\text { (homogenized) }\end{array}$ & 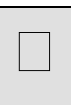 & & & 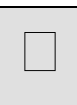 & 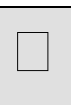 & 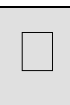 & \\
\hline Cheese & $\square$ & $\square$ & $\square$ & $\square$ & $\square$ & $\square$ & $\square$ \\
\hline $\begin{array}{l}\text { Other milk products } \\
\text { (yogurt, chocolate milk, } \\
\text { pudding, etc.) }\end{array}$ & L & & L & 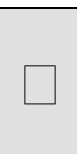 & [ & L & \\
\hline $\begin{array}{l}\text { Whole grain bread or } \\
\text { cereal (oatmeal, muesli, } \\
\text { etc.) }\end{array}$ & $\square$ & 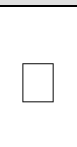 & L & 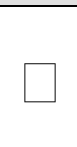 & - & $\square$ & \\
\hline $\begin{array}{l}\text { Meat alternatives (beans, } \\
\text { lentils, tofu, eggs, peanut } \\
\text { butter, etc.) }\end{array}$ & $\square$ & L & $\square$ & $\square$ & - & $\square$ & ] \\
\hline $\begin{array}{l}\text { Energy drinks (Red Bull, } \\
\text { Rock Star, Guru, etc.) }\end{array}$ & $\square$ & $\square$ & $\square$ & $\square$ & $\square$ & $\square$ & $\square$ \\
\hline
\end{tabular}




\begin{tabular}{|l|c|c|c|c|c|c|c|}
\hline $\begin{array}{l}\text { Sports drinks (Gatorade, } \\
\text { Powerade, etc.) }\end{array}$ & $\square$ & $\square$ & $\square$ & $\square$ & $\square$ & $\square$ & $\square$ \\
\hline Fish & $\square$ & $\square$ & $\square$ & $\square$ & $\square$ & $\square$ & $\square$ \\
\hline Ice cream & $\square$ & $\square$ & $\square$ & $\square$ & $\square$ & $\square$ & $\square$ \\
\hline $\begin{array}{l}\text { Fried food such as chicken } \\
\text { wings, chicken fingers, } \\
\text { etc. }\end{array}$ & $\square$ & $\square$ & $\square$ & $\square$ & $\square$ & $\square$ & $\square$ \\
\hline $\begin{array}{l}\text { Fast foods such as pizza, } \\
\text { hamburgers, etc. }\end{array}$ & $\square$ & $\square$ & $\square$ & $\square$ & $\square$ & $\square$ & $\square$ \\
\hline
\end{tabular}

33. How many times do you usually eat the following food items while watching television?

\begin{tabular}{|c|c|c|c|c|c|c|c|}
\hline & Never & $\begin{array}{c}\text { Less than } \\
\text { once a } \\
\text { week }\end{array}$ & $\begin{array}{c}\text { Once a } \\
\text { week }\end{array}$ & $\begin{array}{c}2-4 \\
\text { days a } \\
\text { week }\end{array}$ & $\begin{array}{l}5-6 \\
\text { days a } \\
\text { week }\end{array}$ & $\begin{array}{c}\text { Once a } \\
\text { day, } \\
\text { every } \\
\text { day }\end{array}$ & $\begin{array}{l}\text { Every } \\
\text { day, } \\
\text { more } \\
\text { than } \\
\text { once }\end{array}$ \\
\hline $\begin{array}{l}\text { Potato chips or } \\
\text { peanuts }\end{array}$ & $\square$ & $\square$ & $\square$ & $\square$ & $\square$ & $\square$ & $\square$ \\
\hline $\begin{array}{l}\text { Fried food such as } \\
\text { chicken wings, } \\
\text { chicken fingers, } \\
\text { french fries, etc. }\end{array}$ & 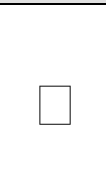 & $\square$ & & & $\square$ & & \\
\hline $\begin{array}{l}\text { Cookies, biscuits, } \\
\text { chocolate or candy } \\
\text { bars }\end{array}$ & $\square$ & $\square$ & $\square$ & $\square$ & $\square$ & $\square$ & $\square$ \\
\hline Ice cream & $\square$ & $\square$ & $\square$ & $\square$ & $\square$ & $\square$ & $\square$ \\
\hline $\begin{array}{l}\text { Fast foods such as } \\
\text { pizza, hamburgers, } \\
\text { etc. }\end{array}$ & $\square$ & $\square$ & $\square$ & $\square$ & $\square$ & $\square$ & $\square$ \\
\hline
\end{tabular}




\begin{tabular}{|l|l|l|l|l|l|l|l|}
\hline Fruits or vegetables & $\square$ & $\square$ & $\square$ & $\square$ & $\square$ & $\square$ & $\square$ \\
\hline
\end{tabular}

34. How often do you usually have breakfast (more than a glass of milk or fruit juice)? Mark one box for weekdays and one box for weekend.

\section{Weekdays}

$\square$ I never have breakfast on weekdays

One day

$\square$ Two days

$\square$ Three days

$\square$ Four days

Five days

\section{Weekend}

$\square$ ever have breakfast on the weekend

$\square$ I usually have breakfast on only one day of the weekend (Saturday OR Sunday) I usually have breakfast on both weekend days (Saturday AND Sunday)

35. Does your school serve school lunches?

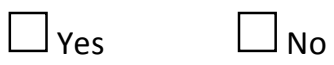

36. In the last week you were in school, about how many times a week did you eat a school lunch?
$\square 0$ days
$\square$ day
$\square 2$ days
$\square 3$ days
$\square 4$ days
$\square 5$ days

37. During thepast week, how many meals (breakfast, lunch or dinner) did you get that were prepared away from home in places such as restaurants, fast food places, food stands, grocery stores or vending machines? (please do not include meals provided as part of school breakfast or school lunch)

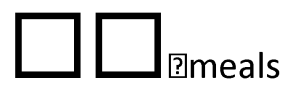

How well do these statements describe you? (Put a mark in the box that best describes how often this happens) 


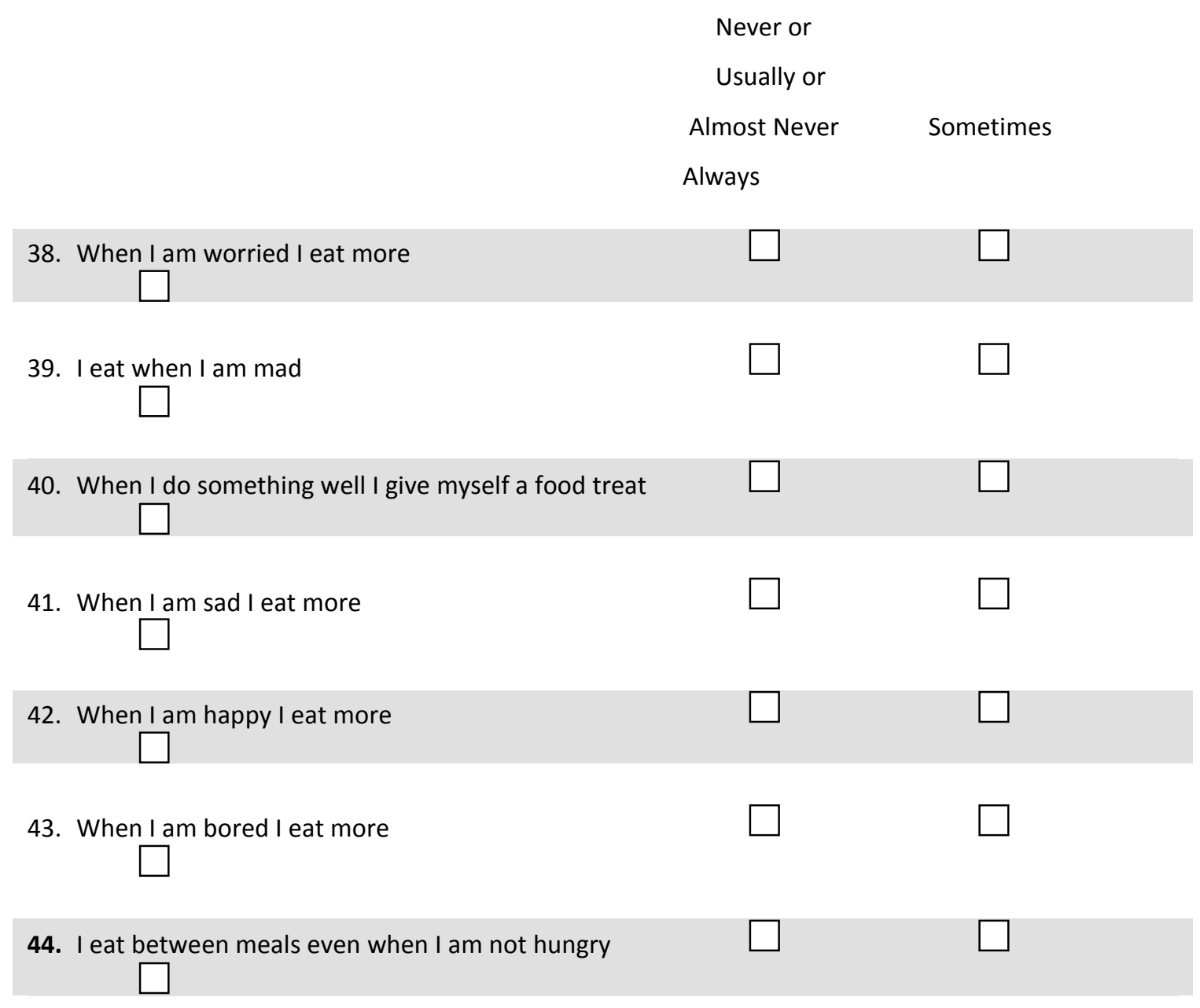

Thinking about the last week... (Put a mark in the box that best describes how you felt)

$\begin{array}{lll}\text { Not at all } & \text { Slightly } & \text { Moderately } \\ \text { Very } & \text { Extremely } & \end{array}$

45. Have you felt fit and well?

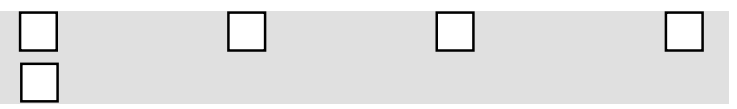

46. Have you felt full of energy?

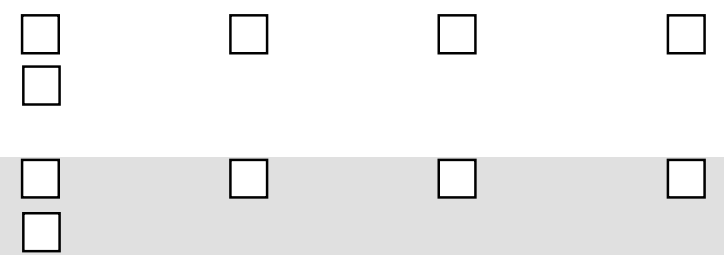

47. Have you felt sad? 
48. Have you felt lonely?

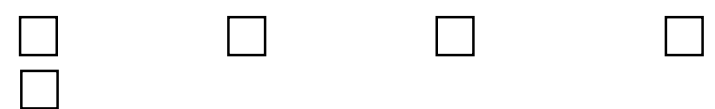

49. Have you had enough time for yourself?

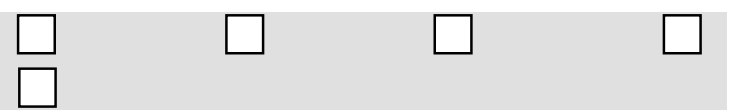

50. Have you been able to do the things

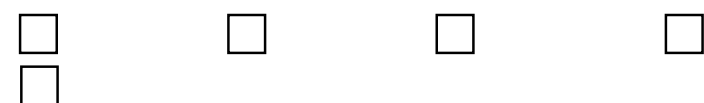

that you want to do in your free time?

51. Have your parent(s) treated you fairly?

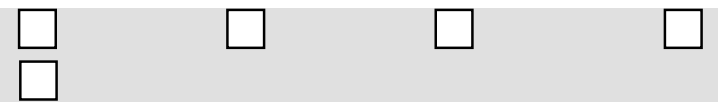

52. Have you had fun with your friends?

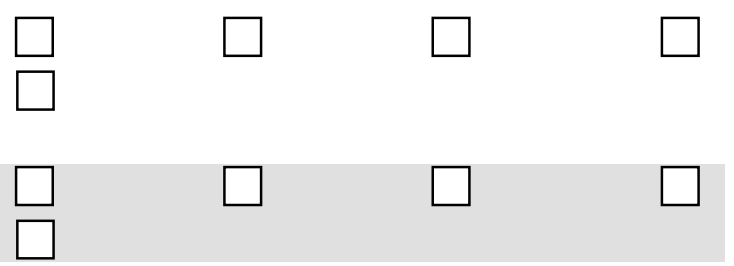

54. Have you been able to pay attention?
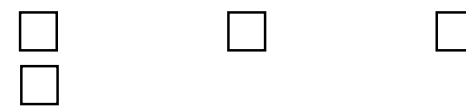

55. In general, how would you say your health is?

$\square$ excellent $\square$ very good $\quad \square$ good $\quad \square_{\text {fair }} \quad \square$ poor

\section{Thank you! ()}




\section{Appendix E}

\section{ISCOLE Demographic and Family Health Questionnaire}

\section{A. GENERAL INFORMATION}

Child's Name:

Last $\quad$ First $\quad$ Middle

Name of Child's School:

Parent's or Guardian's Name:

Last

First

Middle

Home Address:

Street Address Apt.\# Town or City Province Postal/Zip Code

Nearest Cross-Street to Home:

Phone Number: ( )

E-Mail:

Area Code

How long have you lived at the current address?

years and

months

\section{B. DEMOGRAPHICS OF CHILD}

Birth date

Age years Gender: Male Female

$\mathrm{dd} / \mathrm{mmm} / \mathrm{yyyy}$

Ethnicity ${ }^{1}$ :

${ }^{1}$ The researchers are interested in learning about the ethnicity of your child because several studies show that individuals from various parts of the world present a different susceptibility to weight gain. The question is not intended to discriminate any child on the basis of ethnicity. Your 
$\square$ White

African American

Asian

$\square$ First Nations

$\square$ East Indian

$\square$ Don't know

$\square$ Other

\section{Are you of Hispanic origin ${ }^{1}$}

$\square \quad$ Yes $\quad \square \quad$ No

In what country was the child born?

How many biological brothers and sisters does the child have?

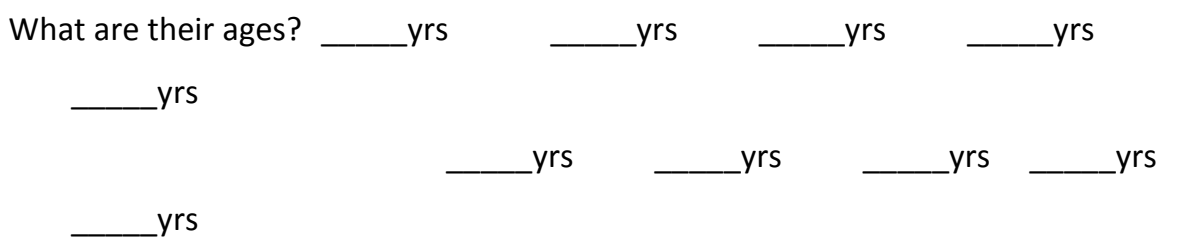

\section{HEALTH HISTORY OF CHILD}

1. Birth Weight: grams OR Ibs \& oz Birth Length: $\mathrm{cm} \mathrm{OR}$ inches

2. Length of Pregnancy: weeks OR months

3. Did mother develop gestational diabetes during pregnancy with THIS child? Yes No

4. Fed breast milk? Yes $\square \quad$ No $\square$ No, please skip to question 5 .

answer will be used in the scientific analyses, only to be able to look into this important issue on a preventive standpoint. If you do not want to answer a question (but remember all answers are kept private and confidential) please draw a line through the question and mark it "PM", meaning Permanently Missing. 
Age when COMPLETELY stopped being fed breast milk: months

Age when FIRST fed formula: months

5. Age when COMPLETELY stopped drinking formula: months

\section{FAMILY DEMOGRAPHICS AND HEALTH}

6. What is the marital status of the child's parents?
Married
Divorced or separated
Never married
Widowed parent

7. How many people live in your household (at this address)?

7a. Who lives with the child atthis address (check all that apply)?

$\begin{array}{lll}\square \text { Biological Mother } & \square \text { Brother(s) or Sister(s) } \\ \square \text { Biological Father } & \square \text { Grandparent(s) } \\ \square \text { Adoptive Mother } & \square \text { Other Relative(s) } \\ \square \text { Adoptive Father } & \square \text { Friend(s) } \\ \square \text { Step Mother } & \square \text { Legal Guardian(s) } \\ \square \text { Step Father } & \square \text { OTHER }\end{array}$

8. What is the COMBINED annual income for your household (before taxes)?
Less than $\$ 14,999$
$\$ 15,000-\$ 29,999$
$\$ 30,000-\$ 39,999$
$\$ 40,000-\$ 59,999$
$\square \$ 60,000-\$ 89,999$
$\$ 90,000-\$ 109,999$
$\$ 110,000-\$ 139,999$
$\square \$ 140,000$ and above

9. How many functioning motorized vehicles (car, truck, motorcycle, moped, etc.) are available for use at your house?

0 


or more

10. How many television sets are in your household?
0
1
2
3
$\square 4$
5 or more

11. What best describes your type of television service for the primary television in the house?

$\square$ No television
$\square$ Antenna only
$\square$ Basic cable
Cable + premium channel(s)
Satellite dish
Other
Don't know

12. What best describes your type of internet service?

No internet access

$\square$ Dial-up modem

$\square$ DSL modem

$\square$ Cable modem

$\square$ Other

Don't know

13. What is the MOTHER'S highest level of education completed?
$\square$ Less than high school
Some high school
High school diploma/GED
Diploma or 1-3 years of college
Bachelor's degree 
Graduate (Master's or PhD)/professional degree

14. How many hours per week does the MOTHER work outside the home?

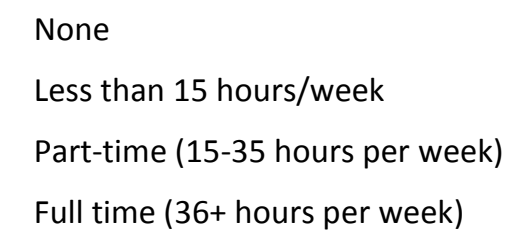

15. What is the FATHER'S highest level of education completed?

Less than high school

Some high school

High school diploma/GED

Diploma or 1-3 years of college

$\square$ Bachelor's degree

Graduate (Master's or PhD)/professional degree

16. How many hours per week does the FATHER work outside the home?

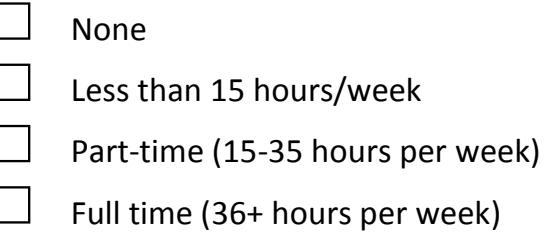

17. Is this child adopted?

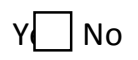

18. Please answer the following questions with regard to the child's BIOLOGICAL MOTHER (if she is pregnant, please report the pre-pregnancy weight):

Current height: $\mathrm{cm}$ or feet and inches

Current weight: kg or Ibs

Current Age: years

Age at child's birth: years

iological Mother's information cannot be estimated or is not known 
19. Please answer the following questions with regard to the child's BIOLOGICAL FATHER:

Current height:

cm or feet and inches Current weight:

kg or Ibs

Current age: years

Ziological Father's information cannot be estimated or is not known 


\section{Appendix F}

\section{ISCOLENeighbourhood \&Home Environment Questionnaire}

Where "child" is mentioned, please respond only about the child who is participating in this study. Be as accurate as you can. There are no right or wrong answers. All information is strictly confidential.

\section{A. NEIGHBOURHOOD COHESION}

Do you agree or disagree with the Strongly Somewhat Neutral Somewhat Strongly following statements?

$$
\text { disagree disagree agree agree }
$$

1. People around my

neighbourhood are willing to

O

0

O

○

O help their neighbours.

2. This is a close-knit neighbourhood.

O

$\bigcirc$

O

O

O

3. People in my neighbourhood can be trusted.

O $\quad 0$

O

O

4. People in my neighbourhood generally don't get along with each other.

\section{People in my neighbourhood do} not share the same values, O O $\bigcirc$ O O attitudes or beliefs.

\section{B. NEIGHBOURS AND FRIENDS}


1. Think about the neighbourhood or area in which you live. In general, how well do you feel you know your neighbours?

$\begin{array}{cccc}\text { Not } & \text { Just } & \text { Moderately } & \text { Extremely } \\ \text { at all } & \text { a little } & \text { well } & \text { well } \\ 0 & 0 & 0 & 0\end{array}$

2. About how often do you talk to or visit with your immediate neighbours (people in the 10-20 households that live closest to you)?

$\begin{array}{ccccccc} & & \text { Several } & & \text { Several } & \text { Several } & \\ \text { Never } & \text { Once a } & \text { times a } & \text { Once a } & \text { times a } & \text { times a } & \text { Almost } \\ 0 & 0 & \text { year } & \text { month } & \text { month } & \text { week } & \text { every day } \\ & 0 & 0 & 0 & 0 & 0\end{array}$




\section{NEIGHBOURHOOD RESPONSE}

For the following statements, please mark Neither how likely a neighbour would respond to, or take action in the following situations:

$\begin{array}{ccc} & \text { Neither } & \\ & \text { likely } & \\ \text { Very } & \text { nor } & \text { Very } \\ \text { unlikely Unlikely } & \text { unlikely Likely likely }\end{array}$

1. If a group of neighbourhood children were skipping school and hanging out on a street corner, how likely is it that your neighbours $0 \quad 0$ ○ $0 \quad 0$ would do something about it?

2. If some children were spray-painting graffiti on a local building, how likely is it that your neighbours would do something about it?

3. If a child was showing disrespect to an adult, how likely is it that people in your O ○ $\bigcirc$ ○ 0 neighbourhood would scold that child?

4. If there was a fight in front of your house and someone was being beaten or threatened, how likely is it that your neighbours would break it up?

\section{Suppose that because of budget cuts the fire} station closest to your home was going to be closed down by the city. How likely is it that neighbourhood residents would $0 \quad 0$ organize to try to do something to keep the fire station open?

\section{FOODS IN THE HOME}

How often are the following foods/drinks available in your home?

Never Rarely Sometimes Often Always
1. Chocolate candy
○
$\bigcirc$
$\bigcirc$
O
O
2. Other candy
O
O
O

$0 \quad 0$




\begin{tabular}{|c|c|c|c|c|c|}
\hline 3. Raw fruit (e.g., apples, oranges) & O & O & O & O & O \\
\hline 4. Cakes, brownies, muffins or cookies & O & O & ○ & $\mathrm{O}$ & $\mathrm{O}$ \\
\hline 5. Regular chips or crackers & O & O & O & $\bigcirc$ & O \\
\hline 6. Baked chips, low-fat crackers, pretzels & $\mathrm{O}$ & O & ○ & O & O \\
\hline 7. Raw vegetables (e.g., carrots) & ○ & ○ & $\bigcirc$ & O & O \\
\hline 8. $100 \%$ fruit juice & O & O & ○ & $\mathrm{O}$ & $\mathrm{O}$ \\
\hline 9. Juice drinks (e.g., Sunny delight) & O & $\bigcirc$ & O & O & $\mathrm{O}$ \\
\hline 10. Regular sodas with sugar & O & O & O & O & O \\
\hline 11. Diet or sugar free sodas & O & O & O & ○ & $\mathrm{O}$ \\
\hline 12. Sports drinks (e.g., Gatorade) & $\mathrm{O}$ & $\bigcirc$ & O & O & $\mathrm{O}$ \\
\hline 13. Fruit roll-ups or other dried fruit & $\mathrm{O}$ & O & O & $\bigcirc$ & O \\
\hline 14. Regular or $2 \%$ milk & O & $\bigcirc$ & $\bigcirc$ & O & $\mathrm{O}$ \\
\hline 15. $1 \%$ or fat-free milk & $\mathrm{O}$ & $\bigcirc$ & O & $\bigcirc$ & $\mathrm{O}$ \\
\hline 16. Sweetened breakfast cereal & O & O & O & ○ & $\mathrm{O}$ \\
\hline 17. Unsweetened breakfast cereal & $\mathrm{O}$ & O & O & O & O \\
\hline
\end{tabular}




\section{E. WHERE YOU SHOP}

When you, or the main food shopper in your home, go food shopping, how often do you go to each of these types of stores?

Never Rarely Sometimes Often Always

$\begin{array}{llllll}\text { 1. Large supermarket or discount warehouse } & 0 & 0 & 0 & 0 & 0 \\ \text { 2. Small to medium food store } & 0 & 0 & 0 & 0 & 0 \\ \text { 3. Convenience store } & 0 & 0 & 0 & 0 & 0 \\ \text { 4. Farmer's market/produce stand } & 0 & 0 & 0 & 0 & 0 \\ \text { 5. Other, specify: } & 0 & 0 & 0 & 0 & 0 \\ \end{array}$

\section{F. STORE ACCESS}

Please indicate whether the following statements are true of

Not the store where you usually buy groceries.
1. Close to location of my employment
2. Close to my child's school

00
3. Close to my home
$\mathrm{O}$
O
O
○
O
○

Yes No applicable

\section{G. FOOD SHOPPING}

The following questions apply to the store where you usually buy groceries.

$\begin{array}{lccc}\text { Strongly } & \text { Somewhat } & \text { Somewhat } & \text { Strongly } \\ \text { disagree disagree Neutral } & \text { agree } & \text { agree }\end{array}$

1. Low-fat foods cost too much.

$0 \quad 0$

$\bigcirc$

0

$\mathrm{O}$

2. There is a large selection of fresh fruits and vegetables.

3. There is a large selection of lowfat products available.

O $\quad 0$

O

$\bigcirc$

4. The condition of fresh fruits and vegetables is poor.

O $\quad 0$

O

○

5. Fruits and vegetables cost too much.

O $\quad 0$

O

O

O 


\section{H. YOUR CHILD'S ELECTRONICS}

Please indicate whether the following are in your child's bedroom.

$\begin{array}{lll}\text { 1. TV } & 0 & 0 \\ \text { 2. Computer } & 0 & 0\end{array}$

3. Video game system (non-hand held; Playstation, Xbox, etc.) $\bigcirc$

Does your child have the following items for his/her own use?

4. Cell phone or 2-way radio

5. Hand-held videogame players (Game Boy, Sony PSP, etc.)

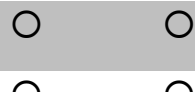

6. Music systems (Ipod, stereo, radio, etc.) 
For the next two questions, please think about your child's activities over the past year.

\section{PLAY EQUIPMENT}

\begin{tabular}{|c|c|c|c|c|c|}
\hline $\begin{array}{l}\text { How often during the past year has } \\
\text { your child used these items at or } \\
\text { around home (or in a common } \\
\text { apartment area)? }\end{array}$ & $\begin{array}{c}\text { Not } \\
\text { available } \\
\text { (Don't } \\
\text { have) }\end{array}$ & $\begin{array}{c}\text { Available } \\
\text { but } \\
\text { never } \\
\text { use }\end{array}$ & $\begin{array}{l}\text { Once a } \\
\text { month } \\
\text { or less }\end{array}$ & $\begin{array}{l}\text { Once } \\
\text { every } \\
\text { other } \\
\text { week }\end{array}$ & $\begin{array}{l}\text { Once a } \\
\text { week or } \\
\text { more }\end{array}$ \\
\hline 1. Bike & 0 & 0 & 0 & 0 & 0 \\
\hline 2. Basketball hoop & O & O & O & O & O \\
\hline 3. Jump rope & 0 & O & 0 & O & O \\
\hline $\begin{array}{l}\text { 4. Active video games (e.g., with } \\
\text { dance pad, Wii, etc) }\end{array}$ & O & O & O & O & 0 \\
\hline $\begin{array}{l}\text { 5. Sports equipment (like ball, } \\
\text { racquets, bats, sticks) }\end{array}$ & O & 0 & O & 0 & 0 \\
\hline 6. Swimming pool & O & O & O & 0 & O \\
\hline $\begin{array}{l}\text { 7. Roller skates, skateboard, } \\
\text { scooter }\end{array}$ & 0 & 0 & 0 & 0 & 0 \\
\hline $\begin{array}{l}\text { 8. Fixed play equipment (e.g., } \\
\text { swing set, playhouse, jungle } \\
\text { gym) }\end{array}$ & O & O & O & 0 & O \\
\hline
\end{tabular}

\section{J. PLACES FOR YOUR CHILD'S PHYSICAL ACTIVITY}

\begin{tabular}{|c|c|c|c|c|c|c|}
\hline \multirow{3}{*}{$\begin{array}{l}\text { How often during the past year has } \\
\text { your child been physically } \\
\text { active(including active play) in the } \\
\text { following places? }\end{array}$} & \multirow{3}{*}{\multicolumn{2}{|c|}{$\begin{array}{c}\text { Once } \\
\text { a } \\
\text { month } \\
\text { or less }\end{array}$}} & \multirow{3}{*}{$\begin{array}{l}\text { Once } \\
\text { every } \\
\text { other } \\
\text { week }\end{array}$} & \multirow[b]{3}{*}{$\begin{array}{c}\text { Once } \\
\text { a } \\
\text { week }\end{array}$} & \multirow[b]{3}{*}{$\begin{array}{c}2 \text { or } 3 \\
\text { times/ } \\
\text { week }\end{array}$} & \multirow{3}{*}{$\begin{array}{c}4 \\
\text { times/ } \\
\text { week } \\
\text { or } \\
\text { more }\end{array}$} \\
\hline & & & & & & \\
\hline & & & & & & \\
\hline 1. Inside your home & 0 & 0 & O & O & O & O \\
\hline $\begin{array}{l}\text { 2. In your yard or common area or in } \\
\text { your driveway }\end{array}$ & O & O & O & O & O & O \\
\hline
\end{tabular}


3. At a neighbour's house, yard, or driveway

$\begin{array}{llllll}0 & 0 & 0 & 0 & 0 & 0\end{array}$

4. In a local street, sidewalk, or vacant lot/field

5. Indoor recreation or exercise facility (public or private; e.g., YMCA/Boys $\quad 0 \quad 0 \quad 0 \quad 0 \quad 0 \quad 0$ \& Girls Club)

6. Beach, lake, river, or creek $\quad 0 \quad 0 \quad 00 \begin{array}{llll}0 & 0 & 0\end{array}$

7. Bike/hiking/walking trails, paths $000 \begin{array}{lllll}0 & 0 & 0 & 0\end{array}$

8. Basketball court $\quad 0 \quad 0 \quad 000000$

9. Other playing fields/courts (like

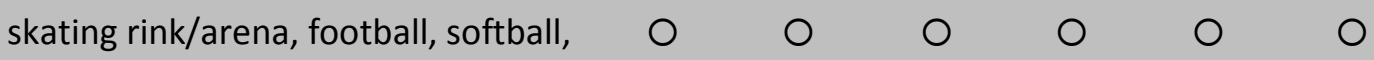
tennis, soccer)

10. Small public park or playground

$\begin{array}{llllll}0 & 0 & 0 & 0 & 0 & 0\end{array}$

11. Large public park

$\begin{array}{llll}0 & 0 & 0 & 0\end{array}$

12. Public open space that is not a park

O

$\mathrm{O}$

O

O

00

13. School grounds (during non-school hours)

$\bigcirc$

O

O

O

O 


\section{K. GETTING AROUND IN YOUR NEIGHBOURHOOD}

Please select the answer that best applies to you and your neighbourhood. Within walking distance means within a 10-15 minute walk from your home.

$\begin{array}{cccc}\text { Strongly } & \text { Somewhat } & \text { Somewhat } & \text { Strongly } \\ \text { disagree disagree } & \text { agree } & \text { agree }\end{array}$

1. There are shops, stores, markets, and places to buy things I need within easy

○

○

○ walking distance of my home/house.

2. There is a bus, subway, or train stop within walking distance from my home.

3. There are sidewalks on most streets. O O O

4. There are NOT many dead end streets.

O

$\bigcirc$

$\bigcirc$

5. There are many different routes for getting from place to place.

$0 \quad 0$ O

6 . There is a high crime rate.

○

O

O

7. The speed of traffic on most streets is usually slow ( $50 \mathrm{~km} / \mathrm{h}$ or less).

O $\quad 0$

O

O

8. Most drivers go faster than the posted speed limits.

9. There are many interesting things to look at while walking in my neighbourhood.

O

$\bigcirc$

$\bigcirc$

$\bigcirc$

10. The traffic makes it difficult or unpleasant for my child to walk.

11. Streets have good lighting at night.

$\mathrm{O}$

O

O

12. There are crosswalks and signals on busy streets.

13. There are many places to go within easy walking distance of my home.
$\bigcirc$
$\bigcirc$

O

O

14. I'm afraid of my child being taken or hurt by a stranger on local streets. 
15. I'm afraid of my child being taken or hurt

by a stranger in my yard, driveway, or

O

O

○

O common area.

16. I'm afraid of my child being taken or hurt by a stranger in a local park.

17. I'm afraid of my child being taken or hurt

by a known "bad" person (adult or child)

O

○

O

in my neighbourhood.

\section{DISTANCE TO LOCATIONS}

About how long would it take youto walk from your home to the nearest places listed below? Please select the time it would take you to walk to each place, regardless of whether you/your child go there.

$\begin{array}{rrrrrr} & & 11- & 21- & & \\ 1-5 & 6-10 & 20 & 30 & 31+ & \text { Don't } \\ \text { min } & \min & \min & \min & \min & \text { know }\end{array}$

1. Convenience/corner store/small grocery store/bodega

$\begin{array}{lllll}0 & 0 & 0 & 0 & 0\end{array}$

2. Supermarket

$\begin{array}{llllll}0 & 0 & 0 & 0 & 0 & 0\end{array}$

3. Fast food restaurant

0000

4. Non-fast food restaurant

O

5. Indoor recreation or exercise facility (public or private; e.g., YMCA/Boys \& Girls Club)

$\begin{array}{llllll}0 & 0 & 0 & 0 & 0 & 0\end{array}$

6. Beach, lake, river, or creek

$\begin{array}{llllll}0 & 0 & 0 & 0 & 0 & 0\end{array}$

7. Bike/hiking/walking trails, paths $0 \quad 0$

8. Basketball court (including half-court)

9. Other playing fields/courts (like skating

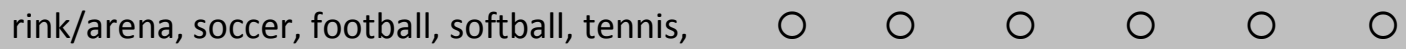
skate park, etc.)

10. Small public park

11. Large public park

$\begin{array}{llllll}0 & 0 & 0 & 0 & 0 & 0\end{array}$

$\begin{array}{lllll}0 & 0 & 0 & 0 & 0\end{array}$


12. Public playground with equipment

$\begin{array}{llllll}0 & 0 & 0 & 0 & 0 & 0\end{array}$

13. School with recreation facilities open to the public
$\bigcirc$
$\bigcirc$
○
O
O
O

\section{FAMILY}

During a typical week, how often do you or another adult in the household:

\begin{tabular}{|c|c|c|c|c|c|}
\hline & Never & $\begin{array}{l}1-2 \\
\text { days }\end{array}$ & $\begin{array}{l}3-4 \\
\text { days }\end{array}$ & $\begin{array}{l}5-6 \\
\text { days }\end{array}$ & Everyday \\
\hline $\begin{array}{l}\text { 1. Watch your child participate in physical activity } \\
\text { or sports }\end{array}$ & 0 & 0 & 0 & 0 & 0 \\
\hline $\begin{array}{l}\text { 2. Encourage your child to do sports or physical } \\
\text { activity }\end{array}$ & 0 & 0 & 0 & 0 & 0 \\
\hline $\begin{array}{l}\text { 3. Provide transport to a place where your child } \\
\text { can do physical activity or play sports }\end{array}$ & 0 & 0 & 0 & 0 & 0 \\
\hline $\begin{array}{l}\text { 4. Do a physical activity or play sports with your } \\
\text { child }\end{array}$ & 0 & 0 & 0 & 0 & 0 \\
\hline
\end{tabular}




\section{Appendix G}

\section{ISCOLE research ethics boards}

\begin{tabular}{|c|c|c|}
\hline $\begin{array}{l}\text { Site } \\
\text { ID }\end{array}$ & Country & IRB/Ethics Committee \\
\hline 01 & $\begin{array}{l}\text { United } \\
\text { States }\end{array}$ & $\begin{array}{l}\text { Pennington Biomedical Research Center Institutional Review Board } \\
\text { for Research with Human Subjects }\end{array}$ \\
\hline 02 & $\begin{array}{l}\text { United } \\
\text { Kingdom }\end{array}$ & University of Bath Research Ethics Committee for Health \\
\hline 03 & Australia & University of South Australia Human Research Ethics Committee \\
\hline 04 & Portugal & Ethics Committee University of Porto \\
\hline 05 & $\begin{array}{l}\text { South } \\
\text { Africa }\end{array}$ & $\begin{array}{l}\text { University of Cape Town Health Sciences Faculty Human Research } \\
\text { Ethics Committee }\end{array}$ \\
\hline 06 & Kenya & $\begin{array}{l}\text { Kenyatta University Ethics Review Committee } \\
\text { Children's Hospital of Eastern Ontario Research Ethics Board }\end{array}$ \\
\hline 08 & Colombia & Universidad de los Andes Committee on Research Ethics \\
\hline 09 & Brazil & $\begin{array}{l}\text { The Research Ethics Committee (REC) of the Municipal Health of } \\
\text { Sao Caetano do Sul - Prima }\end{array}$ \\
\hline 10 & Canada & Children's Hospital of Eastern Ontario Research Ethics Board \\
\hline 11 & China & $\begin{array}{l}\text { Biomedical Ethics Committee of Tianjin Women's and Children's } \\
\text { Health Center }\end{array}$ \\
\hline 12 & India & $\begin{array}{l}\text { St. John's Medical College \& Hospital Institutional Ethical Review } \\
\text { Board }\end{array}$ \\
\hline 13 & Finland & $\begin{array}{l}\text { The Ethics Committee of the Hospital District of Helsinki and } \\
\text { Uusimaa }\end{array}$ \\
\hline
\end{tabular}




\section{Appendix H}

\section{Canadian Assessment of Physical Literacy physical activity knowledge questionnaire}

Physical Activity Knowledge (2013)

(Canadian Assessment of Physical Literacy)

What grade are you in:

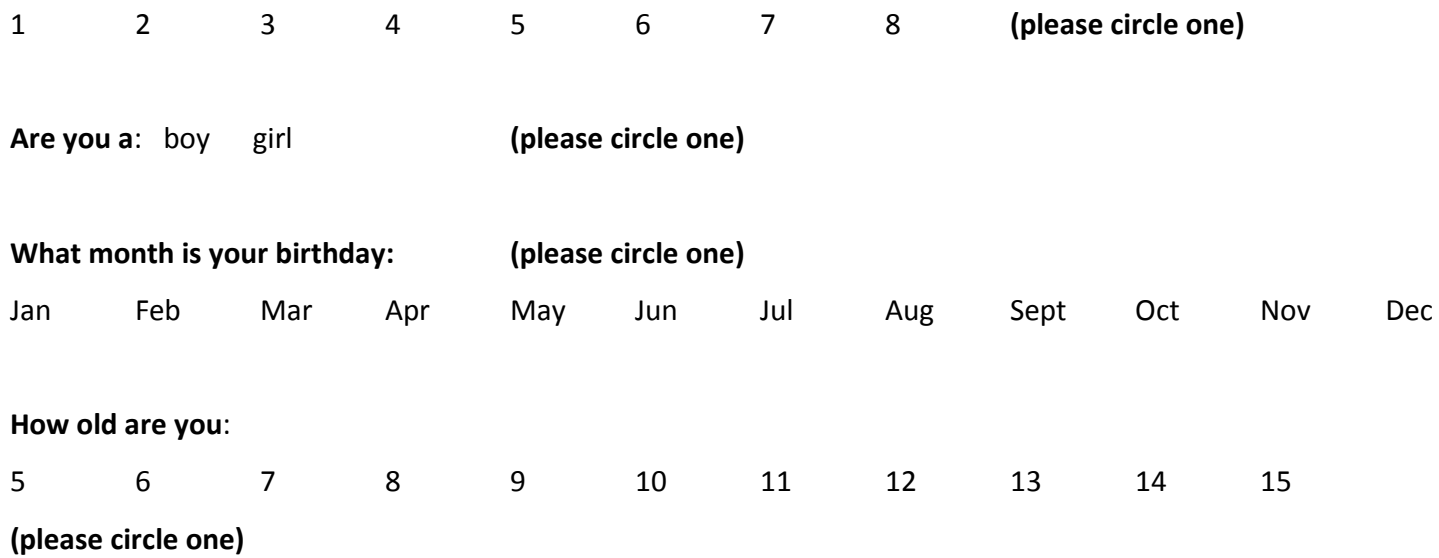

In this project, when we talk about physical activity, we mean when you are moving around, playing or exercising. Physical activity is any activity that makes your heart beat faster or makes you get out of breath some of the time.

Why are we asking you these questions? We want to know what kids like you think about physical activity, sports and exercise.

Please remember:

(i) There are no right or wrong answers. We only want to know what you think.

(i) If you do not know an answer, please write your best guess.

(i) There is no time limit, so please take all the time you need. 
1. Being physically active means you are moving around and not sitting still. How important do you think it is that you are physically active every day?

(circle one number)

Not important

Somewhat important

1

23

$\begin{array}{lll}4 & 5 & 6\end{array}$

7

Very important

2. How important is it for you to be more active than you are now? (circle one number)

I'm already

active enough

1
I need to be a

little more active
I need to be a lot more active

$7 \quad 8$

10

3. Kids say there are many different reasons that they like to be active or play sports. Being active is anything that you do when you are moving, exercising or not sitting still. Below are some reasons that other kids have told us why they like to be active. For each reason, tell us what you think. If you think it is a good reason then you would "Agree a little" or "Agree a lot". If you do not think it's a good reason, then you would "Disagree a little" or "Disagree a lot". If you are not sure or you don't think the reason is good or bad then you are "in between".

\begin{tabular}{|c|c|c|c|c|c|c|}
\hline $\begin{array}{l}\text { A reason that I might be active is } \\
\text { because when I am active } I \text {..... } \\
\text {. }\end{array}$ & $\begin{array}{l}\text { Disagree Disagree } \\
\text { a lot a little }\end{array}$ & $\begin{array}{r}\text { In between } A \\
\text { a little }\end{array}$ & a lot & Agree & & \\
\hline . . look better & 1 & 2 & 3 & & 4 & 5 \\
\hline . . have more energy & 1 & 2 & 3 & & 4 & 5 \\
\hline . . feel happier & 1 & 2 & 3 & & 4 & 5 \\
\hline . . have fun & 1 & 2 & 3 & & 4 & 5 \\
\hline .. make more friends & 1 & 2 & 3 & & 4 & 5 \\
\hline . . get stronger & 1 & 2 & 3 & & 4 & 5 \\
\hline . . like myself more & 1 & 2 & 3 & & 4 & 5 \\
\hline . get in better shape & 1 & 2 & 3 & & 4 & 5 \\
\hline . . feel healthier & 1 & 2 & 3 & & 4 & 5 \\
\hline
\end{tabular}


4. Kids say there are also reasons that make it hard for them to be active. For each reason, tell us what you think. If you think it is a good reason then you would "Agree a little" or "Agree a lot". If you do not think it's a good reason, then you would "Disagree a little" or "Disagree a lot". If you are not sure or you don't think the reason is good or bad then you are "in between".

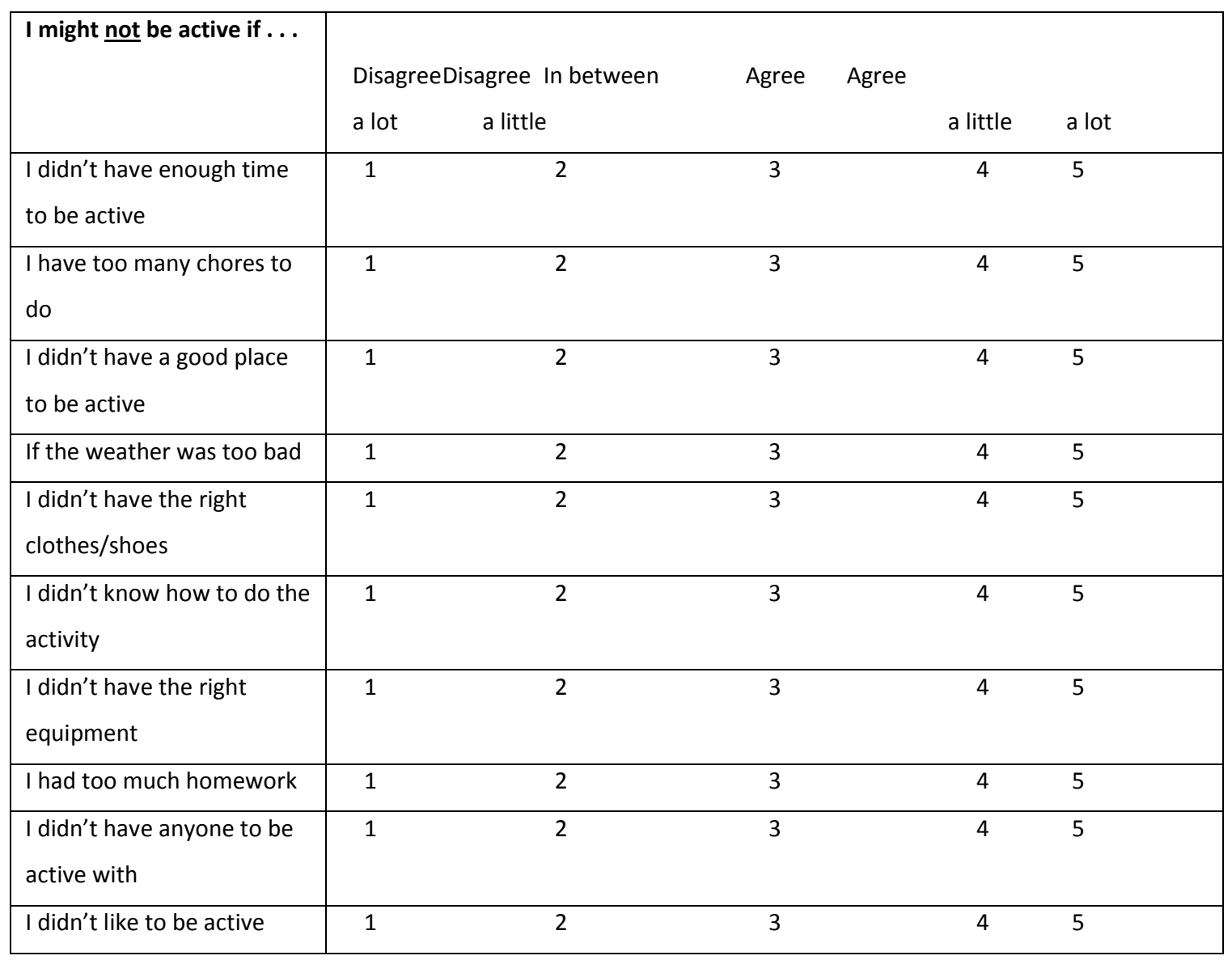

5. Compared to other kids your age, how active are you? (circle one number)

A lot less active Same

A lot more active

$\begin{array}{llllllllll}1 & 2 & 3 & 4 & 5 & 6 & 7 & 8 & 9 & 10\end{array}$

6. Compared to other kids your age, how good are you at sports or skills? (circle one number)

Others are better Same I'm a lot better
12
34
56
$7 \quad 8$
9
10

7. There are many different kinds of fitness. One type is called endurance fitness or aerobic fitness or cardiorespiratory fitness. Cardiorespiratory fitness means...

(circle the right answer)
a) How well the muscles can push, pull or stretch.
b) How well the heart can pump blood and the lungs can provide oxygen.
c) Having a healthy weight for our height.
d) Our ability to do sports that we like.

8. Activities that make your heart beat faster and make you breathe faster, like walking fast or running, are called moderate or vigorous activity. How many minutes of moderate or vigorous physical activity are students supposed to do every day at school? 

a) 10 minutes
b) 20 minutes
c) 30 minutes
d) 60 minutes or 1 hour

9. How many minutes of moderate or vigorous activity should you and other Canadian children do physical activity every day? Count the time you should be active at school and also the time you should be active at home or in your neighbourhood.
a) 10 minutes
b) 20 minutes
C) 30 minutes
d) 60 minutes or 1 hour

10. Sometimes children have to sit still to read, watch television or do homework. What is the most time that children should sit still each day? Do not count the time that you are asleep at night.
a) $\quad 30$ minutes
b) 60 minutes or 1 hour
c) 2 hours
d) $\quad 4$ hours 
11. Draw a line to all the words you think describe what "Healthy" means.

Not being sick

Exercising

Eating well

Being skinny

Being attractive

Strong muscles

Having endurance

12. All of the athletes in the photos below are doing the same skill. What are they all doing? What is the same in every picture?

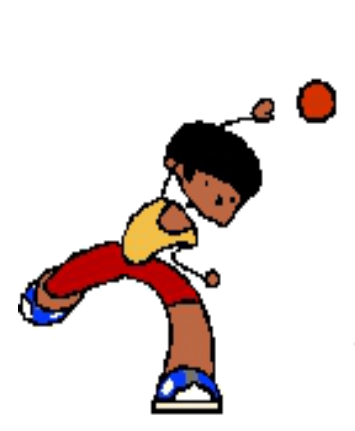

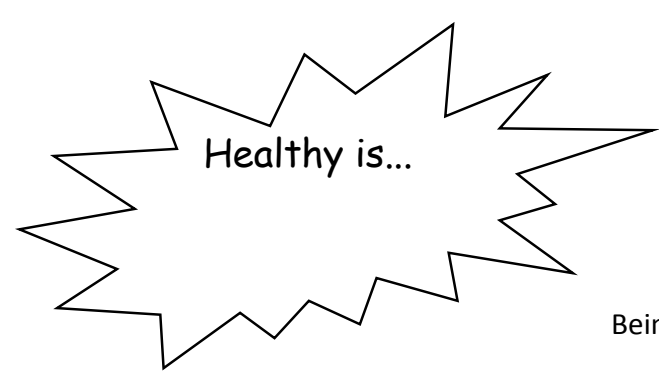

Being happy looking good

feeling good

Being flexible 
13. This story about Sally is missing some words. Fill in the missing words below. Each word can only be used to fill one blank space in the story.

\begin{tabular}{|lccc|}
\hline $\begin{array}{ccc}\text { Cardiorespiratory } \\
\text { Good }\end{array}$ & Fun & Endurance & Heart \\
Pulse & Strength & Lungs \\
\hline
\end{tabular}

Sally goes for a 30 minute jog each day which increases her fitness. Running every day is good for

her and

Sally thinks that physical activity is and is also for her so she participates in at least minutes a day. At her sport team's practice she does more running to improve her The team also does exercises like push-ups and sit-ups that increase her

After exercising, she checks her heart rate which is also

called a

14. In the summer, when you are outside for at least $\mathbf{3 0}$ minutes, how often do you wear sunscreen? (circle one answer)
a) Always
b) Often
c) Sometimes
d) Not very often
e) Never

15. In the winter, when you are outside for at least $\mathbf{3 0}$ minutes, how often do you wear sunscreen? (circle one answer)
a) Always
b) Often
c) Sometimes
d) Not very often
e) Never

16. Please circle all of the foods that are good to eat most days. Put an $X$ through the foods that you should only eat as a special treat.
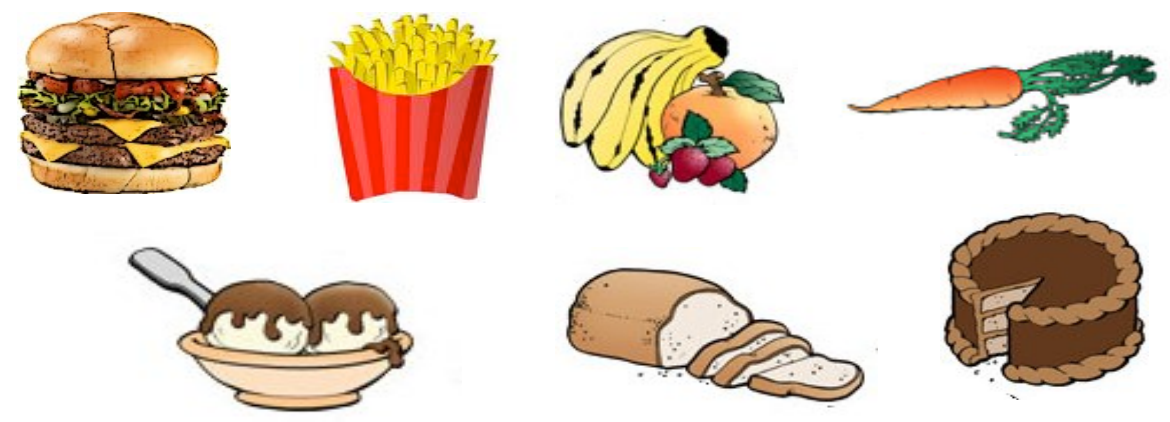
17. Circle each activity that you do. If you always or almost always wear safety gear (like helmet or shin pads) when you do the activity, add a check mark inside the circle.

18. How many hours do you usually spend sleeping each night?

(circle one answer)
a) Less than 5 hours
b) 5 to 8 hours
c) 8 to 10 hours
d) 10 to 12 hours
e) More than 12 hours
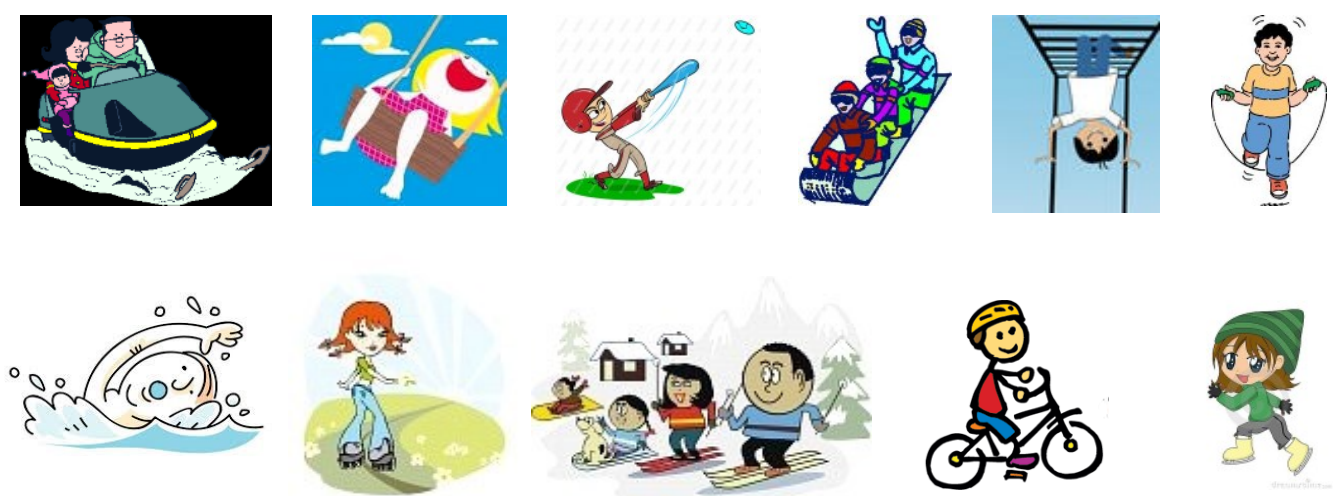
19. During a normal week, how much do your parents do the following things with you:

\begin{tabular}{|l|l|l|l|l|l|}
\hline Take you to play games or sports & Never & Not often & Sometimes & Often & Very Often \\
\hline Be active with me & Never & Not often & Sometimes & Often & Very Often \\
\hline Encourage me to be active or play & Never & Not often & Sometimes & Often & Very Often \\
\hline Play games or sports with me & Never & Not often & Sometimes & Often & Very Often \\
\hline $\begin{array}{l}\text { Play sports with the whole family } \\
\text { Tell me that I am a good person } \\
\text { for being active }\end{array}$ & Never & Not often & Sometimes & Often & Very Often \\
\hline $\begin{array}{l}\text { Tell me that they don't like me } \\
\text { being active }\end{array}$ & Never & Not often & Sometimes & Often & Very Often \\
\hline
\end{tabular}

20. During a normal week, how much do your friends do the following things with you:

\begin{tabular}{|l|l|l|l|l|l|}
\hline Play games or sports with me & Never & Not often & Sometimes & Often & Very Often \\
\hline Be active with me & Never & Not often & Sometimes & Often & Very Often \\
\hline Encourage me to be active or play & Never & Not often & Sometimes & Often & Very Often \\
\hline $\begin{array}{l}\text { Tell me that I'm a good person for } \\
\text { being active }\end{array}$ & Never & Not often & Sometimes & Often & Very Often \\
\hline $\begin{array}{l}\text { Tell me that they don't like me to } \\
\text { be active }\end{array}$ & Never & Not often & Sometimes & Often & Very Often \\
\hline
\end{tabular}


21. If you wanted to get better at a sport skill like kicking and catching a ball, what would be the best thing to do? (circle one answer)

a) Read a book about kicking and catching a ball

b) Wait until you get older

c) Try kicking or catching a lot of times

d) Take a lesson or have a coach teach you how to kick and catch

22. If you wanted to get in better shape, what would be the best thing to do?

(circle one answer)

a) Read a book about getting in shape

b) Wait until you get older

c) Try exercising or being active a lot more

d) Take a lesson or have a coach teach you how to get in shape

23. If you were allowed to pick what you do after school, which activity would you pick? (circle only one activity) Play video/computer games Go to my sports team's practice

Read

Walk my dog

Do homework

Chat with friends online

Play with my friends at the playground

Watch television

24. How much time do you spend on-line, using a computer, emailing, texting, chatting or using a phone:
i. After school?
ii. After dinner?
iii. On weekends?
a. None
a. None
a. None
b. Less than 1 hour
b. Less than 1 hour
b. Less than 1 hour
c. 1 to 2 hours
c. 1 to 2 hours
c. 1 to 2 hours
d. 3 to 4 hours
d. 3 to 4 hours
d. 3 to 4 hours
e. 5 or more
e. 5 or more
e. 5 or more

25. How much time do you spend watching TV, DVDs, or movies, playing video games, reading or doing homework:
i. After school?
ii. After dinner?
iii. On weekends?
a. None
a. None
a. None
b. Less than 1 hour
b. Less than 1 hour
b. Less than 1 hour
c. 1 to 2 hours
c. 1 to 2 hours
c. 1 to 2 hours
d. 3 to 4 hours
d. 3 to 4 hours
d. 3 to 4 hours

e. 5 or more

e. 5 or more

e. 5 or more

(;) Thank you for your help! () 


\section{Appendix I}

\section{Sample SAS code}

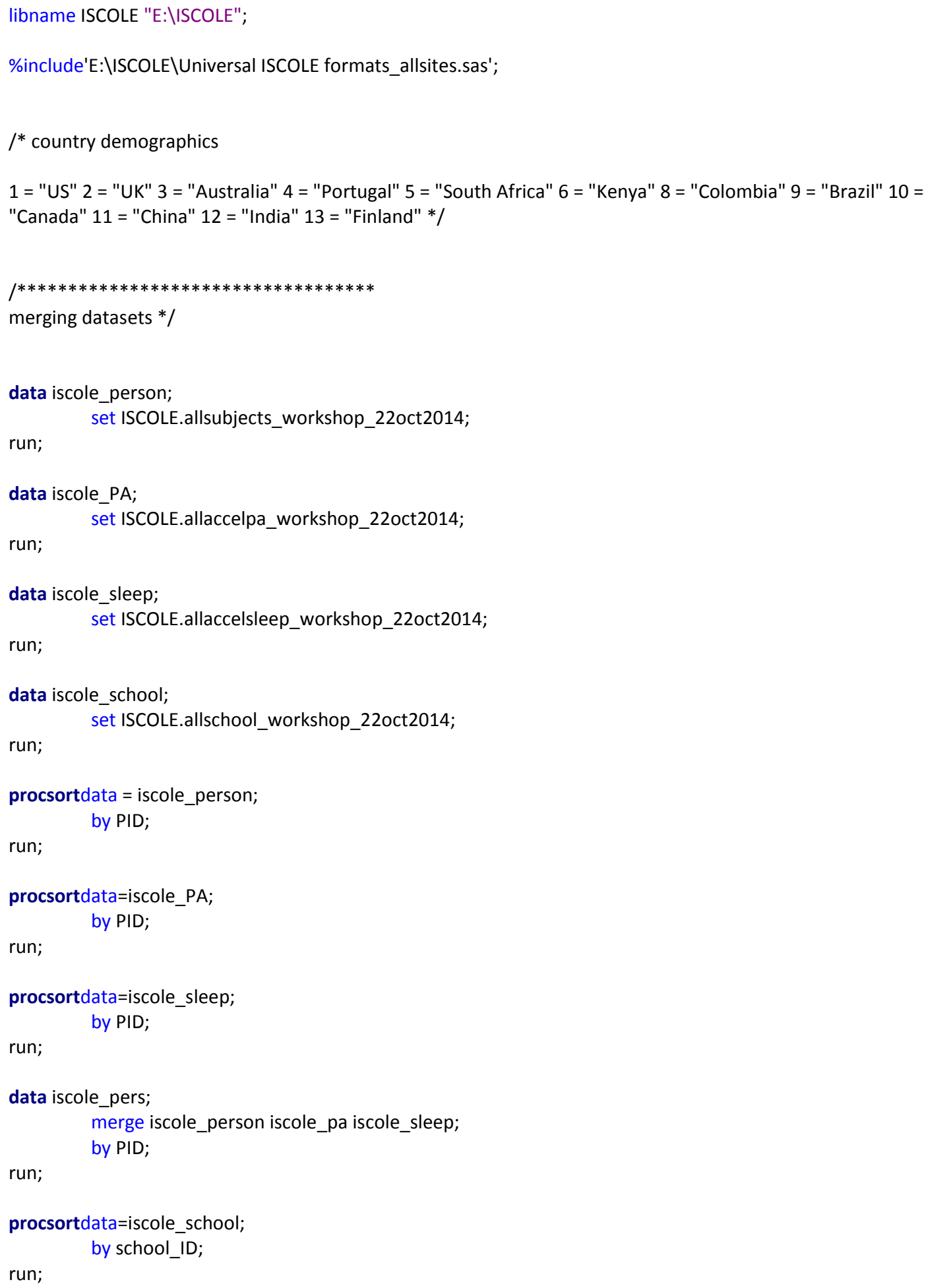


procsortdata= iscole_pers;

by school_ID;

run;

data iscole_complete;

merge iscole_pers iscole_school;

by school_ID;

run;

/*re coding variables */

data iscole_complete1;

set iscole_complete;

$/ *$ Caluculate continuous age in years*/

Age_Year = ageatanthromos/12;

/*Recategorize education*/

if allfather_edu in $(1,2)$ then newfather_edu3 $=1 ; /{ }^{*}$ Did not complete high school*/

if allfather_edu in $(3,4)$ then newfather_edu3 $=2 ; /{ }^{*}$ Completed high school/some college*/

if allfather_edu in $(5,6)$ then newfather_edu3 $=3 ; /{ }^{*}$ Bachelors degree or Postgraduate degree*/

if allmother_edu in $(1,2)$ then newmother_edu3 $=1 ; / *$ Did not complete high school*/

if allmother_edu in $(3,4)$ then newmother_edu3 $=2 ; / *$ Completed high school/some college*/

if allmother_edu in $(5,6)$ then newmother_edu3 $=3 ; / *$ Bachelors degree or Postgraduate degree*/

if allfather_edu in $(1,2,3)$ then newfather_edu2 $=1 ; / *$ Less than or equal to high school diploma*

if allfather_edu in $(4,5,6)$ then newfather_edu2 $=2 ; / *$ Some college/bachelor's/postgrad*/

if allmother_edu in $(1,2,3)$ then newmother_edu2 $=1 ; /{ }^{*}$ Less than or equal to high school diploma* $/$

if allmother_edu in $(4,5,6)$ then newmother_edu2 $=2 ; / *$ Some college/bachelor's/postgrad*/

/*Calculate highest parental education*/

parentschool3 = $\max ($ newfather_edu3, newmother_edu3);

parentschool $2=\max ($ newfather_edu2, newmother_edu2);

run;

data iscole complete2;

set iscole_complete1;

/*Calculate screen time scores*/

/*School day TV*/

if diet_schdaywatchTV in (1) then SchoolTV $=0 ; / *$ did not watch*/

if diet_schdaywatchTV in (2) then schooltv $=0.5 ; / *$ watched less than 1 hour*/

if diet_schdaywatchTV in (3) then schooltv $=1 ; /{ }^{*}$ watched 1 hour*/

if diet_schdaywatchTV in (4) then schooltv $=2 ; / *$ watched 2 hours $* /$

if diet_schdaywatchTV in (5) then schooltv $=3 ; /{ }^{*}$ watched 3 hours $* /$

if diet_schdaywatchTV in (6) then schooltv $=4 ; / *$ watched 4 hours $* /$

if diet_schdaywatchTV in (7) then schooltv $=5 ; / *$ watched 5 hours $* /$

/*Weekend TV*/

if diet_wkendwatchTV in (1) then WkendTV $=0 ; /{ }^{*}$ did not watch $* /$

if diet_wkendwatchTV in (2) then WkendTV $=0.5 ; /{ }^{*}$ watched less than 1 hour*/

if diet_wkendwatchTV in (3) then WkendTV $=1 ; / *$ watched 1 hour*/

if diet_wkendwatchTV in (4) then WkendTV $=2 ; / *$ watched 2 hours*/

if diet wkendwatchTV in (5) then WkendTV $=3 ; /{ }^{*}$ watched 3 hours*/

if diet_wkendwatchTV in (6) then WkendTV $=4 ; / *$ watched 4 hours*/ 


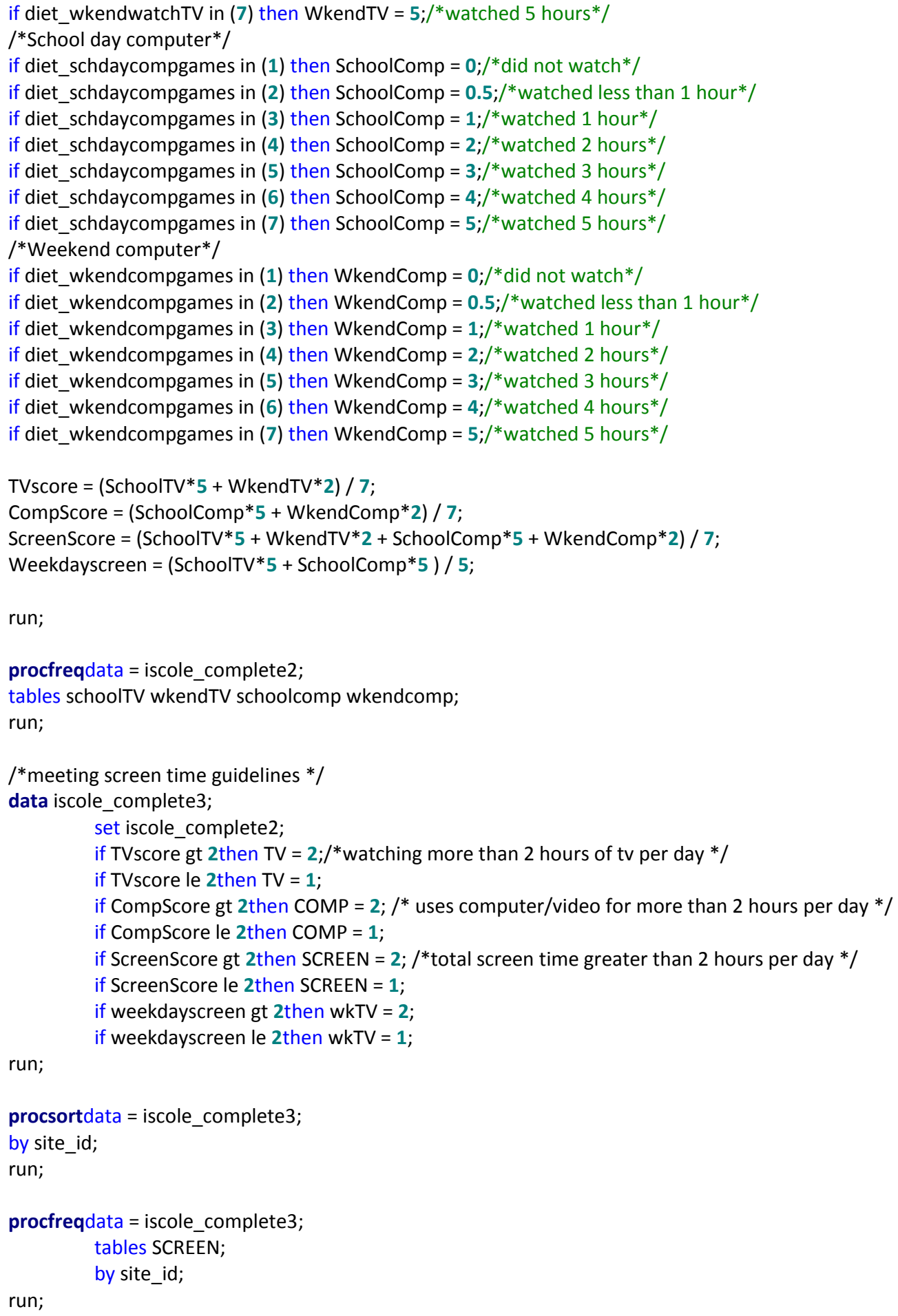




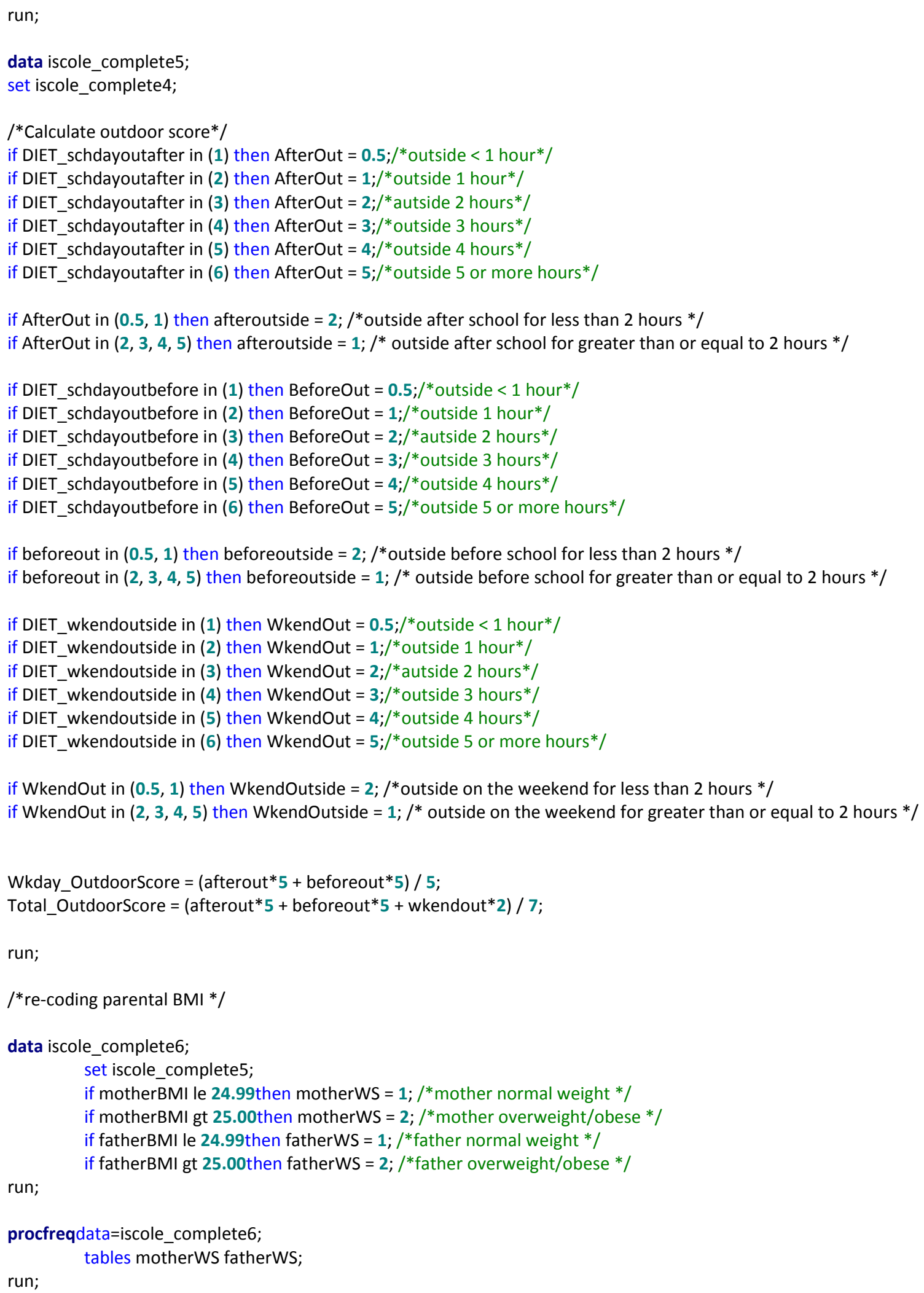


data iscole_complete7;

set iscole_complete6;

if diet_wkratesleep in $(1,2)$ then rate_sleep $=1 ;$ /*sleep quality was very good or fairly good */

if diet_wkratesleep in $(3,4)$ then rate_sleep $=2$; /*sleep quality was fairly bad or very bad */

if diet_wkhowmuchsleep in $(1,2)$ then quantity_sleep $=1$; /*sleep quantity was very good or fairly good*/

if diet_wkhowmuchsleep in $(3,4)$ then quantity_sleep $=2$; /*sleep quantity was fairly bad or very bad */

if diet_7days60mindays in $(1,2,3,4,5,6)$ then guidelines $=2 ; / *$ not meeting PA guidelines */

if diet_7days60mindays in $(7,8)$ then guidelines $=1 ; / *$ meeting guidelines */

if NHEQ_Trusted in $(1,2)$ then trusted $=2 ; / *$ neighbourhood cannot be trusted*/

if NHEQ_Trusted in $(3,4,5)$ then trusted $=1 ; /{ }^{*}$ neighbourhood CAN be trusted*/

if NHEQ_GetManyPIWalk in $(1,2)$ then walking $=2 ; / *$ there are not many places to walk*/

if NHEQ_GetManyPIWalk in $(3,4)$ then walking $=1 ; / *$ there ARE places to walk */

if NHEQ_gethighcrime in $(1,2)$ then crime $=2 ; / *$ high crime $* /$

if NHEQ_gethighcrime in $(3,4)$ then crime $=1 ; / *$ not high crime*/

if NHEQ_elecTV in (1) then parent_TV $=2 ;$ /*parent reports that child has a TV in their room */

if NHEQ_elecTV in (2) then parent_TV $=1 ; / *$ parent reports no TV in bedroom */

if NHEQ_elecCOMP in (1) then parent_comp $=2 ; / *$ parent reports a comp in child's bedroom */

if NHEQ_elecCOMP in (2) then parent_comp $=1 ; /$ * parent reports NO comp in child's bedroom */

if allmother_work in $(2,1)$ then mom_work $=2 ; / *$ mom works less than part time */

if allmother_work in $(3,4)$ then mom_work $=1 ; / *$ mom works part or full time */

if allfather_work in $(1,2)$ then dad_work $=2 ; / *$ dad works less than part time */

if allfather_work in $(3,4)$ then dad_work $=1$; /* dad works part or full time */

${ }^{*}$ Calculate highest parental education*/

parentwork = max (mom_work, dad_work);

parentwork2 = max (allfather_work, allmother_work);

if BMIWHO in $(1,2,3)$ then weight $=1 ; / *$ child under or normal weight */

if BMIWHO in $(4,5)$ then weight $=2 ; / *$ child overweight or obese */

if automobiles le 2then car $=1$;

if automobiles ge 3then car $=2$;

if TVs in $(0,1)$ then TV_house $=2 ; /$ *zero or one TV in house*/

if TVs in $(2,3,4,5)$ then TV_house $=1 ; / * 2$ or more TVs*/

if DIET_TVbedroom in (0) then child_TV $=1 ; / *$ child reports that they don't have a TV in their bedroom */ if DIET_TVbedroom in (1) then child_TV $=2 ; /{ }^{*}$ child reports that they DO have a TV in their room */

run;

$\mid * * * * * * * * * * * * * * * * * * * * * * * * * * * * * * * * * * * * * * * * * /$

procsortdata $=$ iscole_complete7;

by site_id;

run;

data check; /*checking for missing data*/

set iscole_complete7;

if parentwork =. or screenscore $=$. or allmean_sE1=.or avg_weight $=$. or avg_height $=$. or active $=$. or parentschool3 $=$. or motherws $=$. or fatherws $=$. or dietunhealthy_all=. or diethealthy_all=.or quantity_sleep $=$.or rate_sleep $=$. or walking=.

or crime $=$. or $t v \_$house $=$. or car $=$. or diet_tvbedroom $=$. or NHEQ_electv $=$. or NHEQ_eleccomp $=$. or

total_outdoorscore $=$. or trusted=./*or dad_work=. or mom_work=.*/;

run;

data iscole_complete8; /*Then, exclude data that is missing*/ 
set iscole_complete7;

if parentwork=. or screenscore $=$. or allmean_sE1=. or avg_weight $=$. or avg_height $=$. or active $=$. or parentschool $3=$. or motherws $=$. or fatherws $=$. or dietunhealthy_all=. or diethealthy_all=. or quantity_sleep $=$. or rate_sleep $=$. or walking=.

or crime $=$. or tv_house $=$. or car $=$. or diet_tvbedroom $=$. or NHEQ_electv $=$. or NHEQ_eleccomp $=$.or total_outdoorscore=.or trusted=.thendelete;

run;

data iscole_complete8_check; /*then run checks to make sure the code worked.*/

set iscole_complete8;

if parentwork $=$. or screenscore $=$. or allmean_sE1=. or avg_weight $=$. or avg_height $=$. or active $=$. or parentschool $3=$. or motherws $=$. or fatherws $=$. or dietunhealthy_all $=$. or diethealthy_all=.or quantity_sleep $=$.or rate_sleep $=$. or walking $=$.

or crime $=$. or tv_house $=$. or car $=$. or diet_tvbedroom $=$. or NHEQ_electv $=$. or NHEQ_eleccomp $=$.or total_outdoorscore=. or trusted=.;

run;

/* comparing those with missing data */

data iscoleall;

set iscole_complete7;

if gender $=$. or parentwork $=$. or screenscore $=$. or allmean_sE1=. or avg_weight $=$. or avg_height $=$. or active $=$. or parentschool3 $=$.

or motherws $=$. or fatherws $=$. or dietunhealthy_all=.or diethealthy_all=.or quantity_sleep $=$.or rate_sleep=. or walking $=$.

or crime $=$. or tv_house $=$. or car $=$. or diet_tvbedroom $=$. or NHEQ_electv $=$. or NHEQ_eleccomp $=$. or

total_outdoorscore $=$. or trusted $=$. or bmiz_who=.then miss $=1$;

else miss $=0$;

run;

procfreqdata = iscoleall;

tables miss;

run;

/*This will show you if an individual site is missing a lot of data*/

procfreqdata=iscoleall;

tables site_id*miss /chisq;

run;

$/^{*} \mathrm{p}<.05$ means the average of your variable is significantly different between the missing and non-missing groups $* /$ procttestdata=iscoleall;

class miss;

var gender/*insert whichever variable you are supposed to be comparing between missing and non-missing subjects*/;

run;

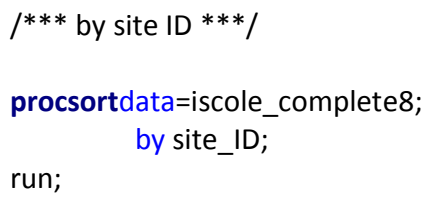




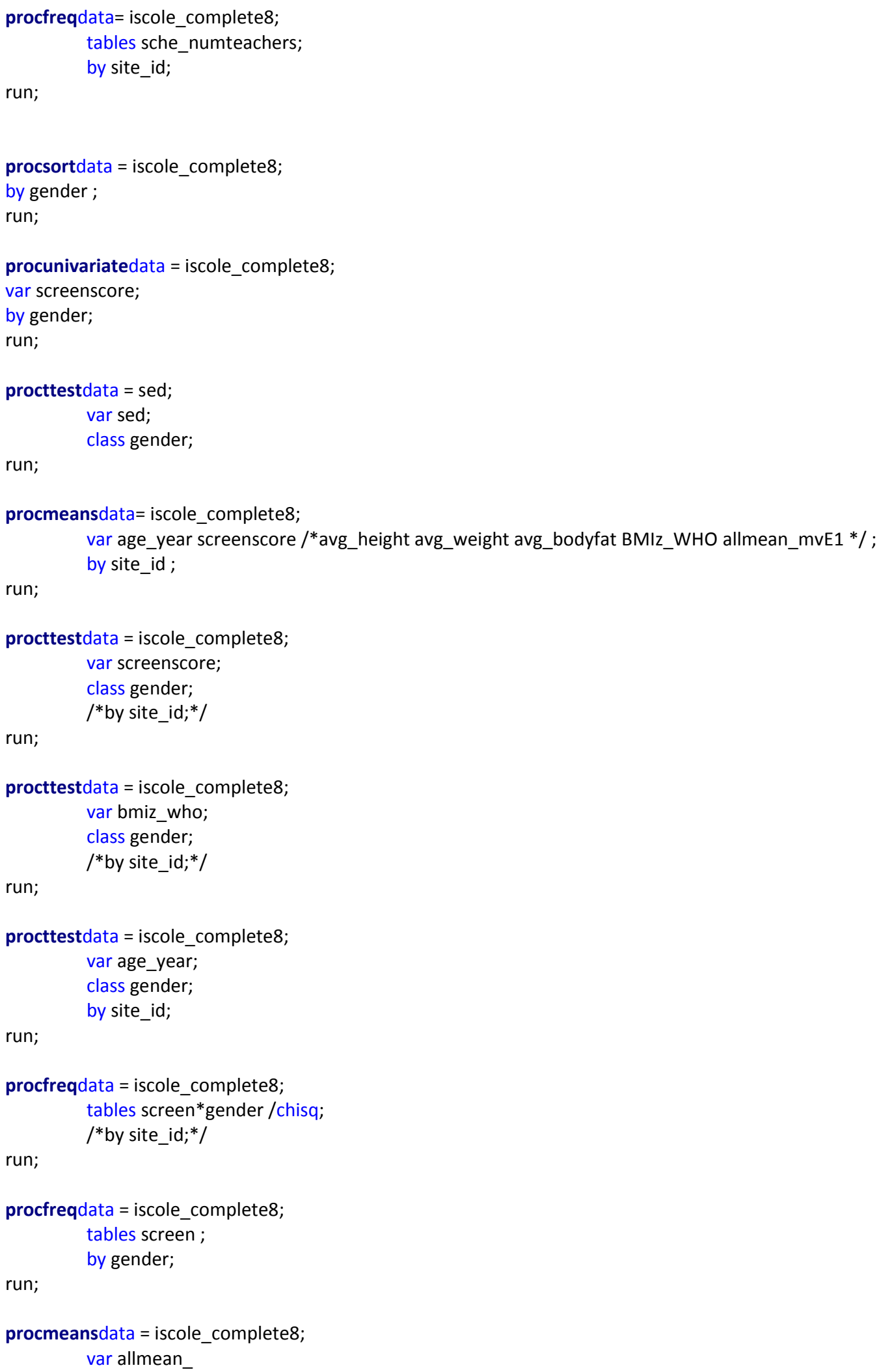




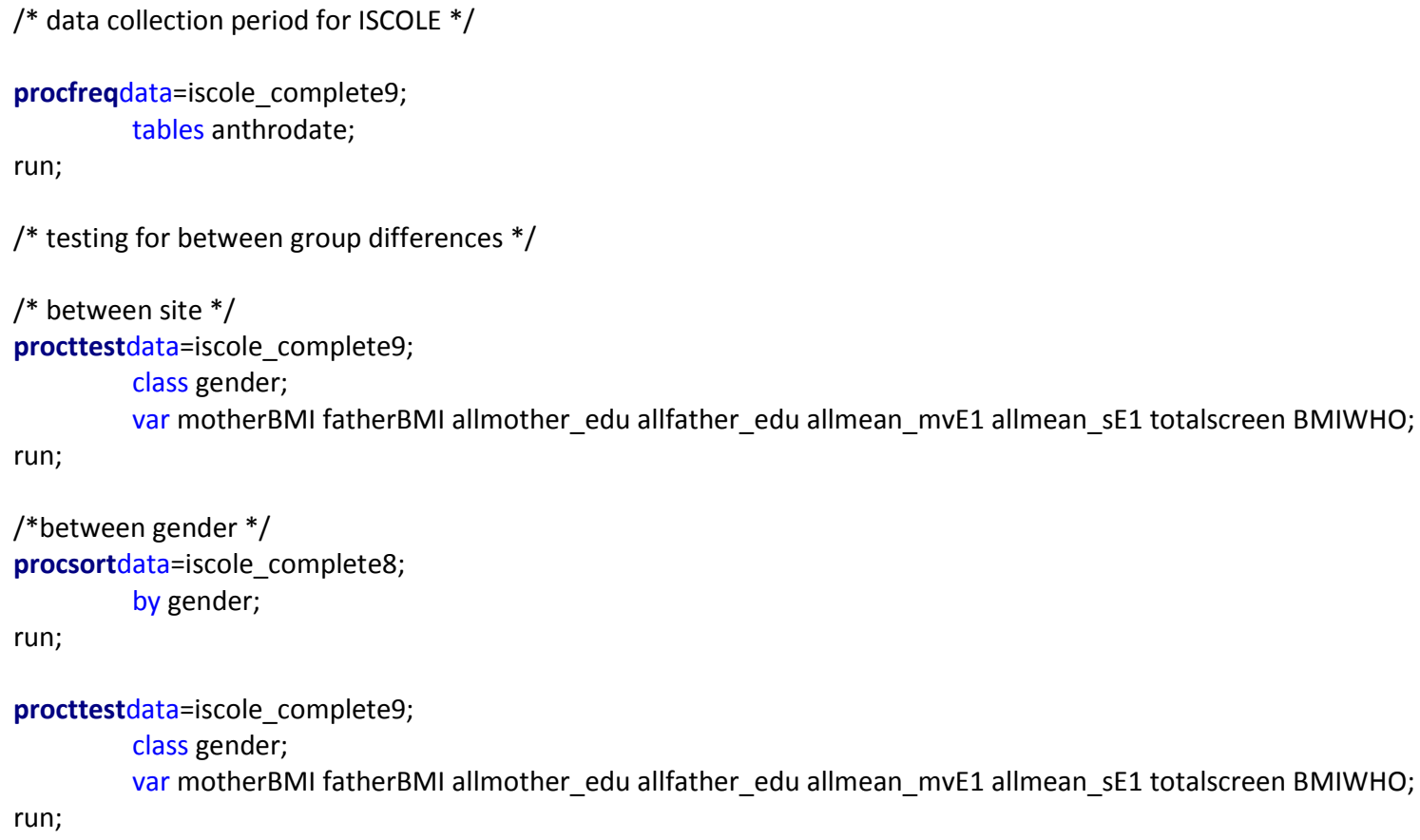

procfreqdata = iscole_complete9; /*Weekend day, how much time did you spend outside?, On a school day, how much time did you spend outside after school before bedtime? On a school day, how much time did you spend outside before school?*/ table DIET_WkEndOutside DIET_SchDayOutAfter DIET_SchDayOutBefore;

run;

procmeansdata $=$ iscole_complete8;

var screenscore;

by/*site_id*/gender;

run;

${ }^{*}$ multilevel

/* calculating ICCs */

procmixeddata=iscole_complete 9 noclprint; class school_ID site_ID ; model allmean_sE1 $=$ /solutionddfm $=\mathrm{kr}$; random intercept $/$ subject $=$ school_ID $($ site_ID); random intercept $/$ subject $=$ site_ID;

run;

procmixeddata=iscole_complete9 noclprint; class school_ID site_ID ; model logscreen $=/$ solutionddfm $=\mathrm{kr}$; random intercept $/$ subject $=$ school_ID (site_ID); random intercept $/$ subject $=$ site_ID;

run;

/************************************************* FINAL model for SED $* * * * * * * * * * /$

procsortdata = iscole_complete9; 
by gender;

run;

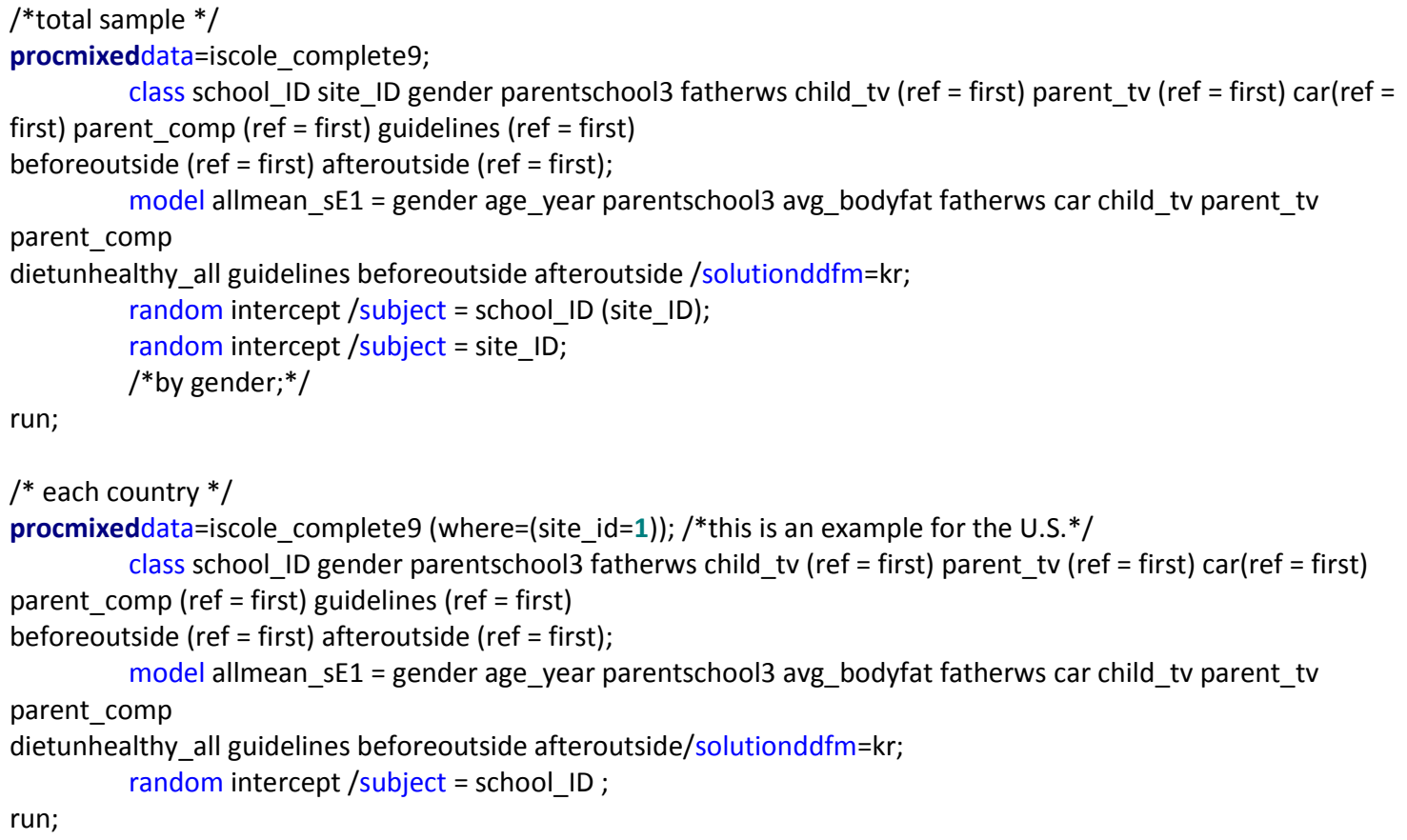

